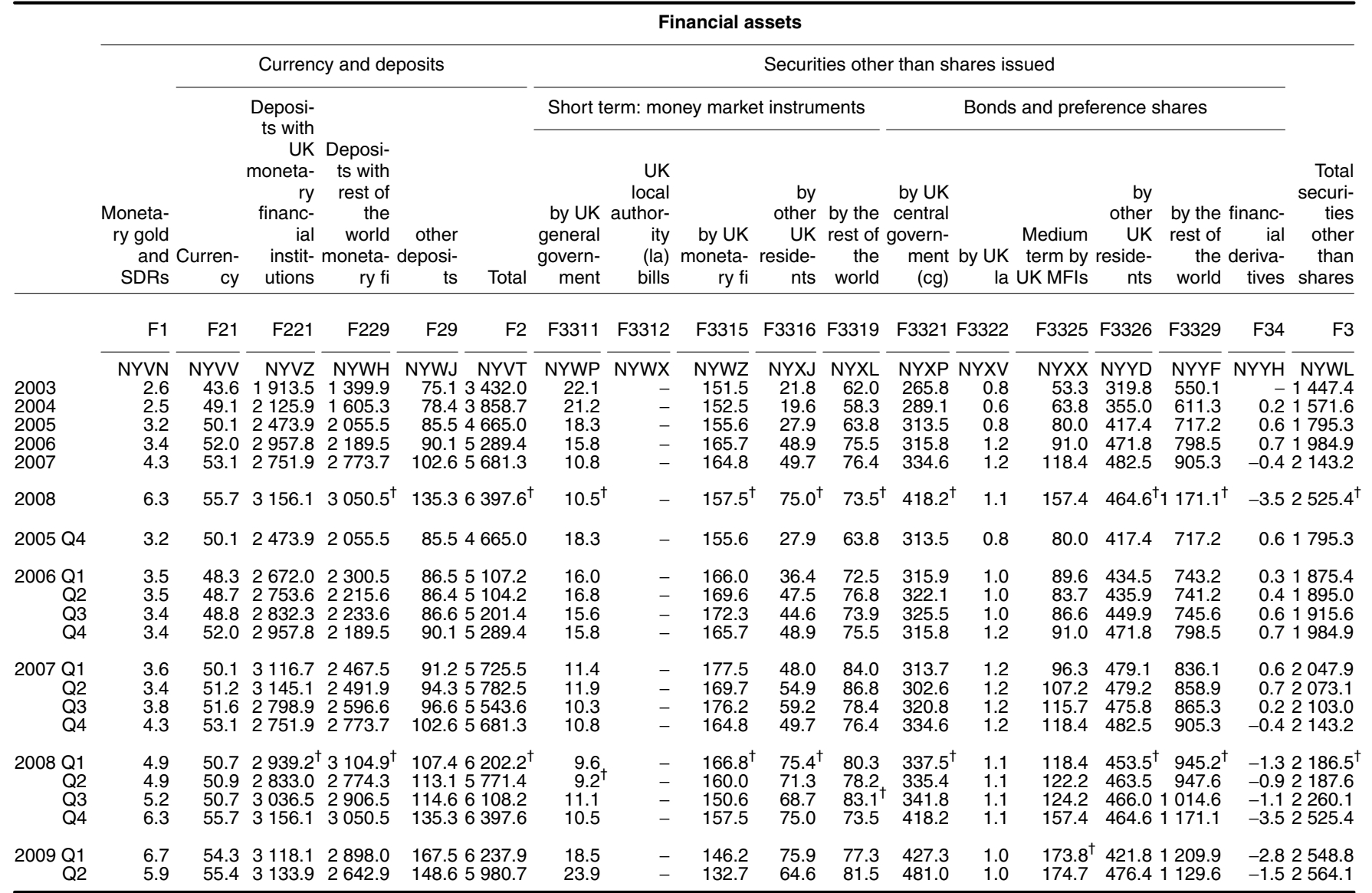

Financial assets - continued

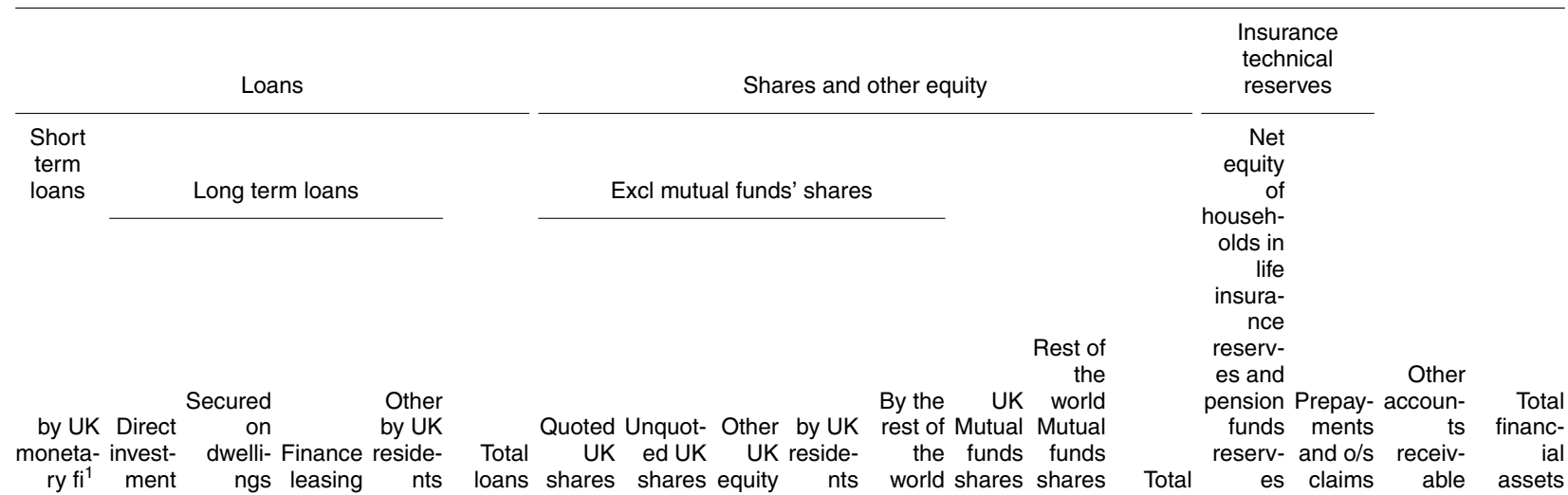

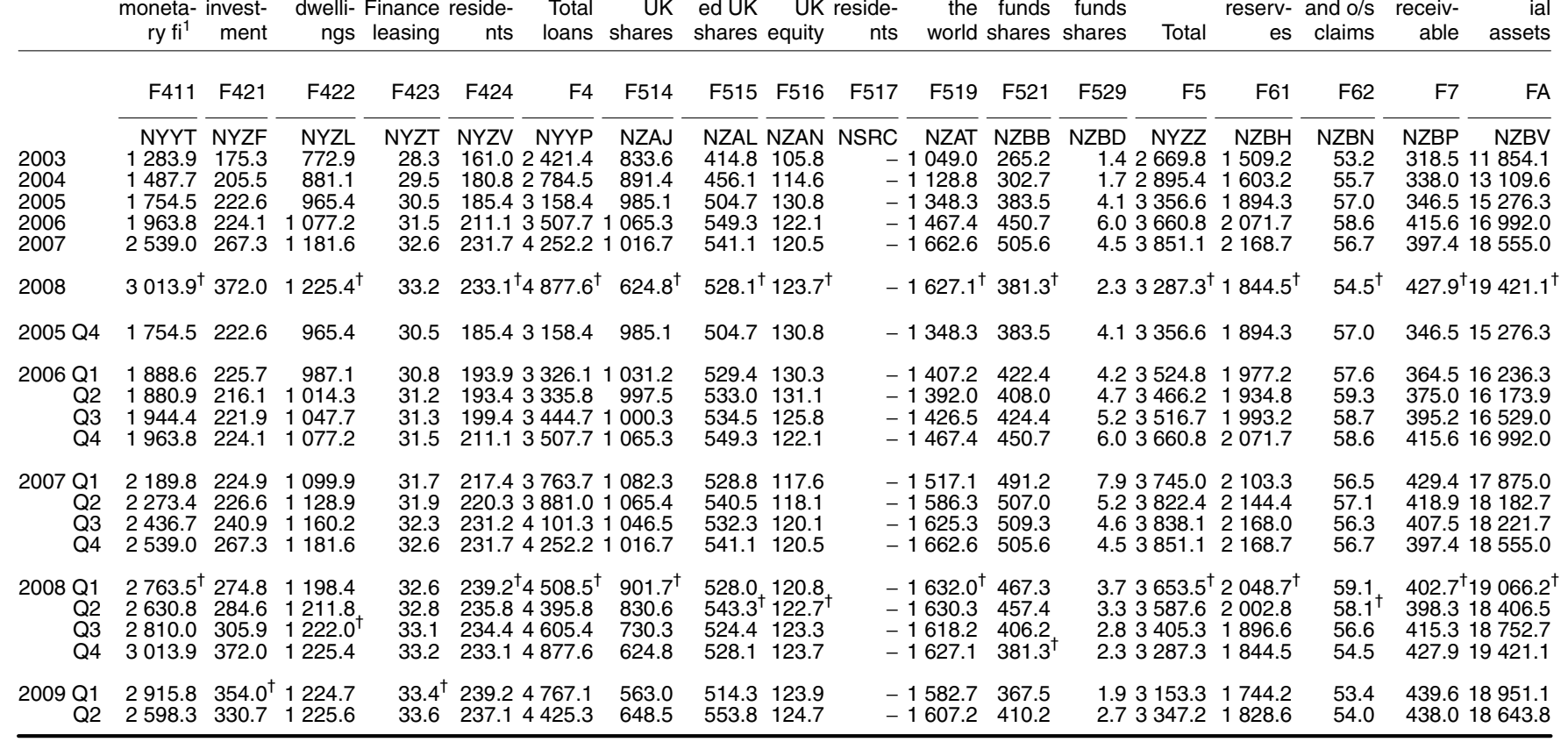




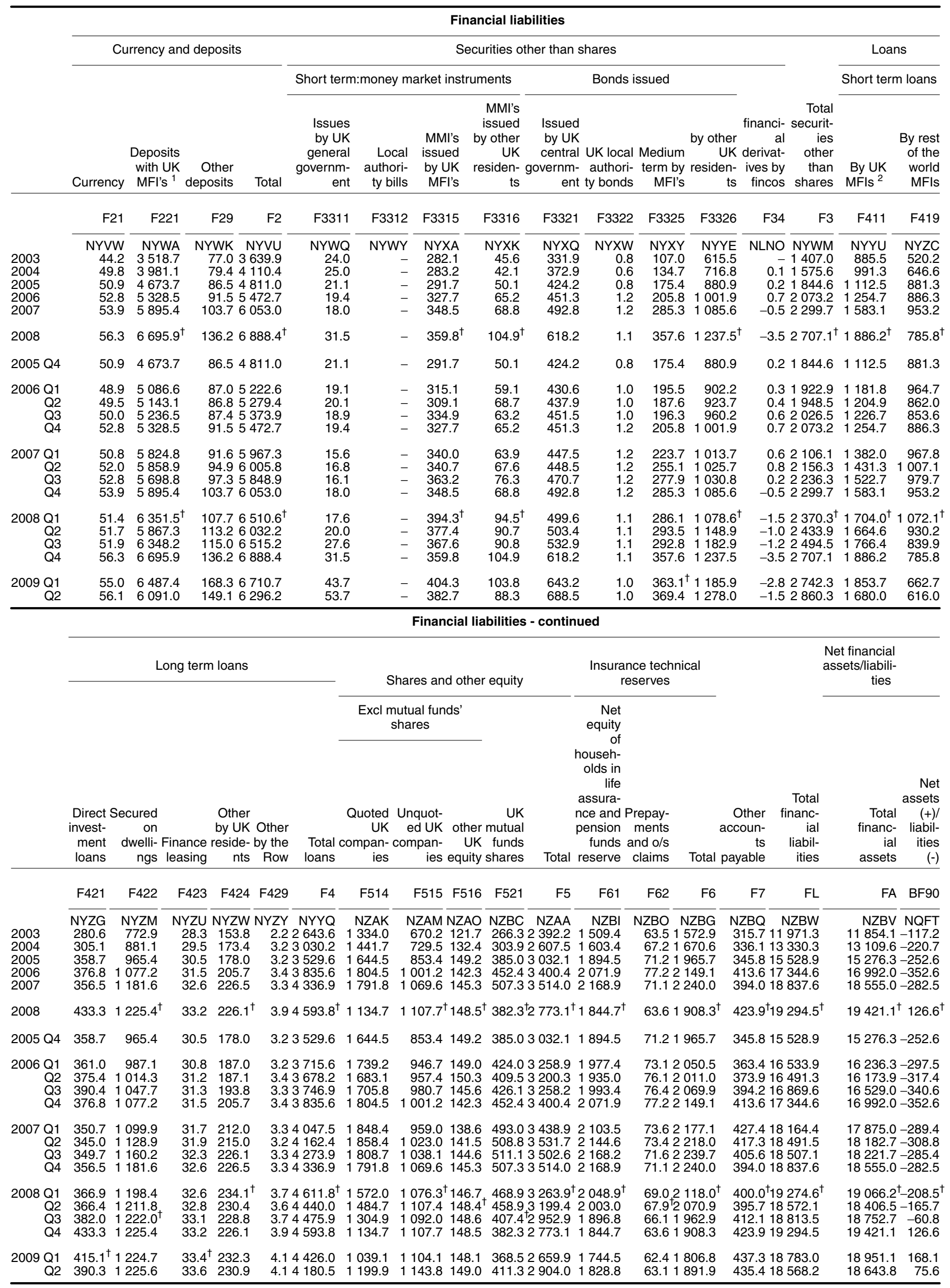




\begin{tabular}{|c|c|c|c|c|c|c|c|c|c|c|c|c|c|c|c|c|}
\hline & \multicolumn{16}{|c|}{ Financial assets } \\
\hline & \multicolumn{5}{|c|}{ Currency and deposits } & \multicolumn{11}{|c|}{ Securities other than shares } \\
\hline & \multirow[b]{2}{*}{ Currency } & \multirow{2}{*}{$\begin{array}{c}\text { Deposits } \\
\text { with UK } \\
\text { MFI's }\end{array}$} & \multirow{2}{*}{$\begin{array}{r}\text { Deposits } \\
\text { with } \\
\text { rest of } \\
\text { the } \\
\text { world } \\
\text { MFl's }\end{array}$} & \multirow{2}{*}{$\begin{array}{r}\text { Deposits } \\
\text { other } \\
\text { than } \\
\text { with } \\
\text { MFl's }\end{array}$} & \multirow[b]{2}{*}{ Total } & \multicolumn{5}{|c|}{ Short term MMl's ${ }^{2}$ issued } & \multicolumn{5}{|c|}{ Bonds issued } & \multirow[b]{2}{*}{ Total } \\
\hline & & & & & & $\begin{array}{r}\text { by UK } \\
\text { general } \\
\text { governm- } \\
\text { ent }\end{array}$ & $\begin{array}{r}\text { UK local } \\
\text { authori- } \\
\text { ty bills }\end{array}$ & $\begin{array}{l}\text { by UK } \\
\text { MFI's }\end{array}$ & $\begin{array}{r}\text { by other } \\
\text { UK } \\
\text { residen- } \\
\text { ts }\end{array}$ & $\begin{array}{l}\text { by the } \\
\text { rest of } \\
\text { the } \\
\text { world }\end{array}$ & $\begin{array}{r}\text { by UK } \\
\text { central } \\
\text { governm- } \\
\text { ent }\end{array}$ & $\begin{array}{r}\text { by UK } \\
\text { local } \\
\text { authori- } \\
\text { ties }\end{array}$ & $\begin{array}{c}\text { Medium } \\
\text { term by } \\
\text { MFl's, }\end{array}$ & $\begin{array}{r}\text { by other } \\
\text { UK } \\
\text { residen- } \\
\text { ts }\end{array}$ & $\begin{array}{l}\text { by the } \\
\text { rest of } \\
\text { the } \\
\text { world }\end{array}$ & \\
\hline & $\mathrm{F} 21$ & F221 & F229 & F29 & $\mathrm{F} 2$ & F3311 & F3312 & F3315 & F3316 & F3319 & F3321 & F3322 & F3325 & F3326 & F3329 & F3 \\
\hline $\begin{array}{l}2002 \\
2003 \\
2004 \\
2005 \\
2006\end{array}$ & $\begin{array}{r}\text { NNZG } \\
3.8 \\
4.1 \\
4.1 \\
4.3 \\
4.5\end{array}$ & $\begin{array}{l}\text { NNZI } \\
177.9 \\
191.4 \\
205.4 \\
230.4 \\
253.6\end{array}$ & $\begin{array}{r}\text { NNZM } \\
65.1 \\
121.1 \\
194.2 \\
255.4 \\
286.9\end{array}$ & $\begin{array}{r}\text { NNZN } \\
8.0 \\
6.7 \\
7.1 \\
8.7 \\
9.4\end{array}$ & $\begin{array}{l}\text { NNZF } \\
254.8 \\
323.4 \\
410.8 \\
498.7 \\
554.4\end{array}$ & $\begin{array}{r}\text { NNZQ } \\
- \\
- \\
- \\
\overline{-} \\
0 . \overline{1}\end{array}$ & $\begin{array}{r}\text { NNZU } \\
- \\
- \\
- \\
- \\
-\end{array}$ & $\begin{array}{r}\text { NNZV } \\
5.0 \\
5.4 \\
5.8 \\
5.2 \\
7.0\end{array}$ & $\begin{array}{r}\text { NOLO } \\
13.9 \\
13.8 \\
13.8 \\
17.9 \\
36.1\end{array}$ & $\begin{array}{r}\text { NOLP } \\
6.0 \\
9.8 \\
10.4 \\
11.4 \\
16.2\end{array}$ & $\begin{array}{r}\text { NOLR } \\
3.5 \\
3.2 \\
3.6 \\
2.7 \\
0.7\end{array}$ & $\begin{array}{r}\text { NOLU } \\
- \\
- \\
- \\
- \\
-\end{array}$ & $\begin{array}{r}\text { NOLV } \\
0.3 \\
0.9 \\
1.0 \\
1.5 \\
1.9\end{array}$ & $\begin{array}{r}\text { NOLY } \\
6.1 \\
5.2 \\
5.1 \\
1.4 \\
1.5\end{array}$ & $\begin{array}{r}\text { NOLZ } \\
29.3 \\
30.0 \\
12.8 \\
14.6 \\
12.1\end{array}$ & $\begin{array}{r}\text { NNZO } \\
64.1 \\
68.2 \\
52.4 \\
54.6 \\
75.5\end{array}$ \\
\hline $\begin{array}{l}2007 \\
2008\end{array}$ & $\begin{array}{l}4.8 \\
4.9^{\dagger}\end{array}$ & $\begin{array}{l}274.3 \\
267.2\end{array}$ & $\begin{array}{l}365.6^{\dagger} \\
433.2^{\dagger}\end{array}$ & $\begin{array}{l}9.5 \\
9.1\end{array}$ & $\begin{array}{l}654.3^{\dagger} \\
714.4^{\dagger}\end{array}$ & $\begin{array}{l}0.4 \\
0.6\end{array}$ & $\begin{array}{l}- \\
-\end{array}$ & $\begin{array}{r}9.8^{\dagger} \\
12.1^{\dagger}\end{array}$ & $\begin{array}{l}38.5 \\
68.4^{\dagger}\end{array}$ & $\begin{array}{l}6.4 \\
1.3\end{array}$ & $\begin{array}{l}1.2 \\
1.9\end{array}$ & $\begin{array}{l}- \\
-\end{array}$ & $\begin{array}{l}2.5 \\
3.6\end{array}$ & $\begin{array}{l}5.2 \\
7.4\end{array}$ & $\begin{array}{l}13.3^{\dagger} \\
18.0^{\dagger}\end{array}$ & $\begin{array}{r}77.3 \\
113.3^{4}\end{array}$ \\
\hline 2005 Q4 & 4.3 & 230.4 & 255.4 & 8.7 & 498.7 & - & - & 5.2 & 17.9 & 11.4 & 2.7 & - & 1.5 & 1.4 & 14.6 & 54.6 \\
\hline $\begin{array}{r}2006 \text { Q1 } \\
\text { Q2 } \\
\text { Q3 } \\
\text { Q4 }\end{array}$ & $\begin{array}{l}4.2 \\
4.5 \\
4.3 \\
4.5\end{array}$ & $\begin{array}{l}234.3 \\
242.5 \\
243.6 \\
253.6\end{array}$ & $\begin{array}{l}282.8 \\
270.2 \\
291.4 \\
286.9\end{array}$ & $\begin{array}{l}8.8 \\
9.0 \\
9.0 \\
9.4\end{array}$ & $\begin{array}{l}530.1 \\
526.2 \\
548.3 \\
554.4\end{array}$ & $\begin{array}{l}0 . \overline{1} \\
0.1 \\
0.1\end{array}$ & $\begin{array}{l}- \\
- \\
- \\
-\end{array}$ & $\begin{array}{l}5.4 \\
6.8 \\
6.8 \\
7.0\end{array}$ & $\begin{array}{l}27.3 \\
37.3 \\
31.6 \\
36.1\end{array}$ & $\begin{array}{l}13.9 \\
15.3 \\
15.1 \\
16.2\end{array}$ & $\begin{array}{l}0.9 \\
0.8 \\
0.7 \\
0.7\end{array}$ & $\begin{array}{l}- \\
- \\
- \\
-\end{array}$ & $\begin{array}{l}2.0 \\
1.7 \\
1.7 \\
1.9\end{array}$ & $\begin{array}{l}0.6 \\
1.0 \\
0.4 \\
1.5\end{array}$ & $\begin{array}{l}15.3 \\
12.9 \\
12.2 \\
12.1\end{array}$ & $\begin{array}{l}65.5 \\
75.8 \\
68.6 \\
75.5\end{array}$ \\
\hline $\begin{array}{r}2007 \text { Q1 } \\
\text { Q2 } \\
\text { Q3 } \\
\text { Q4 }\end{array}$ & $\begin{array}{l}4.5 \\
4.8 \\
4.5 \\
4.8\end{array}$ & $\begin{array}{l}258.5 \\
274.3 \\
267.6 \\
274.3\end{array}$ & $\begin{array}{l}317.0 \\
339.5 \\
345.4 \\
365.6\end{array}$ & $\begin{array}{l}8.8 \\
9.0 \\
9.0 \\
9.5\end{array}$ & $\begin{array}{l}588.8 \\
627.5 \\
626.5 \\
654.3\end{array}$ & $\begin{array}{c}- \\
- \\
\overline{-} \\
0.4\end{array}$ & $\begin{array}{l}- \\
- \\
- \\
-\end{array}$ & $\begin{array}{r}9.3 \\
10.2 \\
10.6 \\
9.8\end{array}$ & $\begin{array}{l}35.0 \\
43.0 \\
45.4 \\
38.5\end{array}$ & $\begin{array}{r}16.0 \\
15.7 \\
10.1 \\
6.4\end{array}$ & $\begin{array}{l}0.6 \\
0.7 \\
0.9 \\
1.2\end{array}$ & $\begin{array}{l}- \\
- \\
- \\
-\end{array}$ & $\begin{array}{l}1.8 \\
1.9 \\
2.6 \\
2.5\end{array}$ & $\begin{array}{l}0.4 \\
6.9 \\
6.6 \\
5.2\end{array}$ & $\begin{array}{l}11.5 \\
12.1 \\
12.8 \\
13.3\end{array}$ & $\begin{array}{l}74.7 \\
90.5 \\
89.0 \\
77.3\end{array}$ \\
\hline $\begin{array}{r}2008 \text { Q1 } \\
\text { Q2 } \\
\text { Q3 } \\
\text { Q4 }\end{array}$ & $\begin{array}{l}4.7 \\
4.9^{\dagger} \\
4.5 \\
4.9\end{array}$ & $\begin{array}{l}275.9^{\dagger} \\
269.3 \\
263.6 \\
267.2\end{array}$ & $\begin{array}{l}431.5^{\dagger} \\
408.1 \\
410.9 \\
433.2\end{array}$ & $\begin{array}{l}9.7 \\
9.8 \\
8.8 \\
9.1\end{array}$ & $\begin{array}{l}721.7^{\dagger} \\
692.1 \\
687.8 \\
714.4\end{array}$ & $\begin{array}{l}0.6 \\
1.4 \\
1.7 \\
0.6\end{array}$ & $\begin{array}{l}- \\
- \\
- \\
-\end{array}$ & $\begin{array}{r}10.5^{\dagger} \\
11.1 \\
9.6 \\
12.1\end{array}$ & $\begin{array}{l}65.2^{\dagger} \\
61.6 \\
58.2 \\
68.4\end{array}$ & $\begin{array}{l}7.9 \\
8.7 \\
5.8 \\
1.3\end{array}$ & $\begin{array}{l}1.0 \\
1.4 \\
1.5 \\
1.9\end{array}$ & $\begin{array}{l}- \\
- \\
- \\
-\end{array}$ & $\begin{array}{l}2.6 \\
2.8 \\
3.2 \\
3.6\end{array}$ & $\begin{array}{l}6.5 \\
6.8 \\
7.0 \\
7.4\end{array}$ & $\begin{array}{l}15.8 \\
16.1 \\
16.7^{\dagger} \\
18.0^{\dagger}\end{array}$ & $\begin{array}{l}110.1^{\dagger} \\
110.1 \\
103.7 \\
113.3\end{array}$ \\
\hline $\begin{array}{r}2009 \text { Q1 } \\
\text { Q2 }\end{array}$ & $\begin{array}{l}4.9 \\
5.0\end{array}$ & $\begin{array}{l}271.4 \\
271.5\end{array}$ & $\begin{array}{l}385.6 \\
356.0\end{array}$ & $\begin{array}{l}9.2 \\
9.0\end{array}$ & $\begin{array}{l}671.0 \\
641.5\end{array}$ & $\begin{array}{l}0.3 \\
0.3\end{array}$ & $\begin{array}{l}- \\
-\end{array}$ & $\begin{array}{l}12.5 \\
11.6\end{array}$ & $\begin{array}{l}70.3 \\
58.1\end{array}$ & $\begin{array}{l}3.6 \\
3.5\end{array}$ & $\begin{array}{l}1.6 \\
1.8\end{array}$ & $\begin{array}{l}- \\
-\end{array}$ & $\begin{array}{l}4.4^{\dagger} \\
4.4\end{array}$ & $\begin{array}{l}6.8^{\dagger} \\
6.3^{\circ}\end{array}$ & $\begin{array}{l}17.2 \\
18.6\end{array}$ & $\begin{array}{l}116.6 \\
104.6\end{array}$ \\
\hline
\end{tabular}

Financial assets - continued

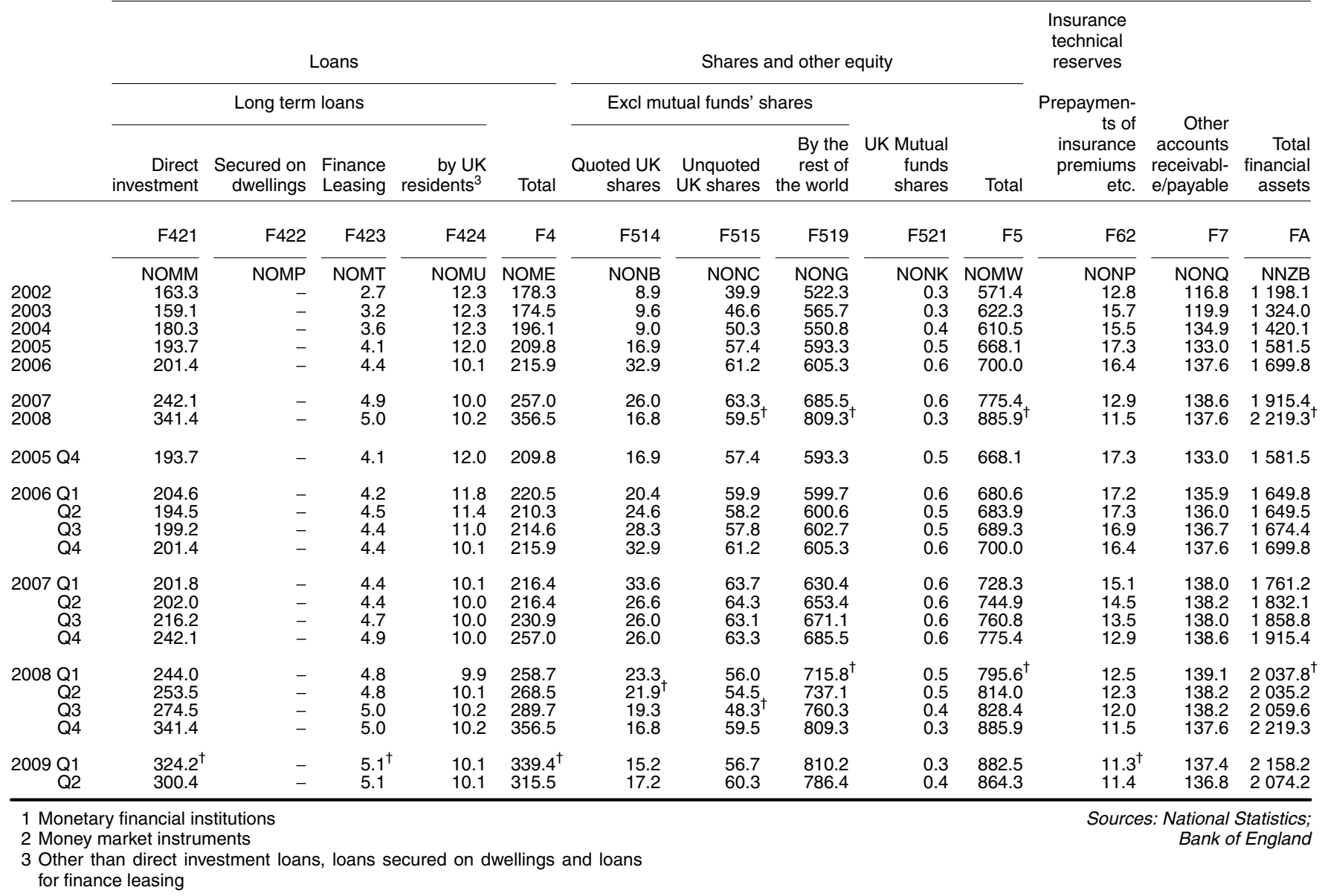




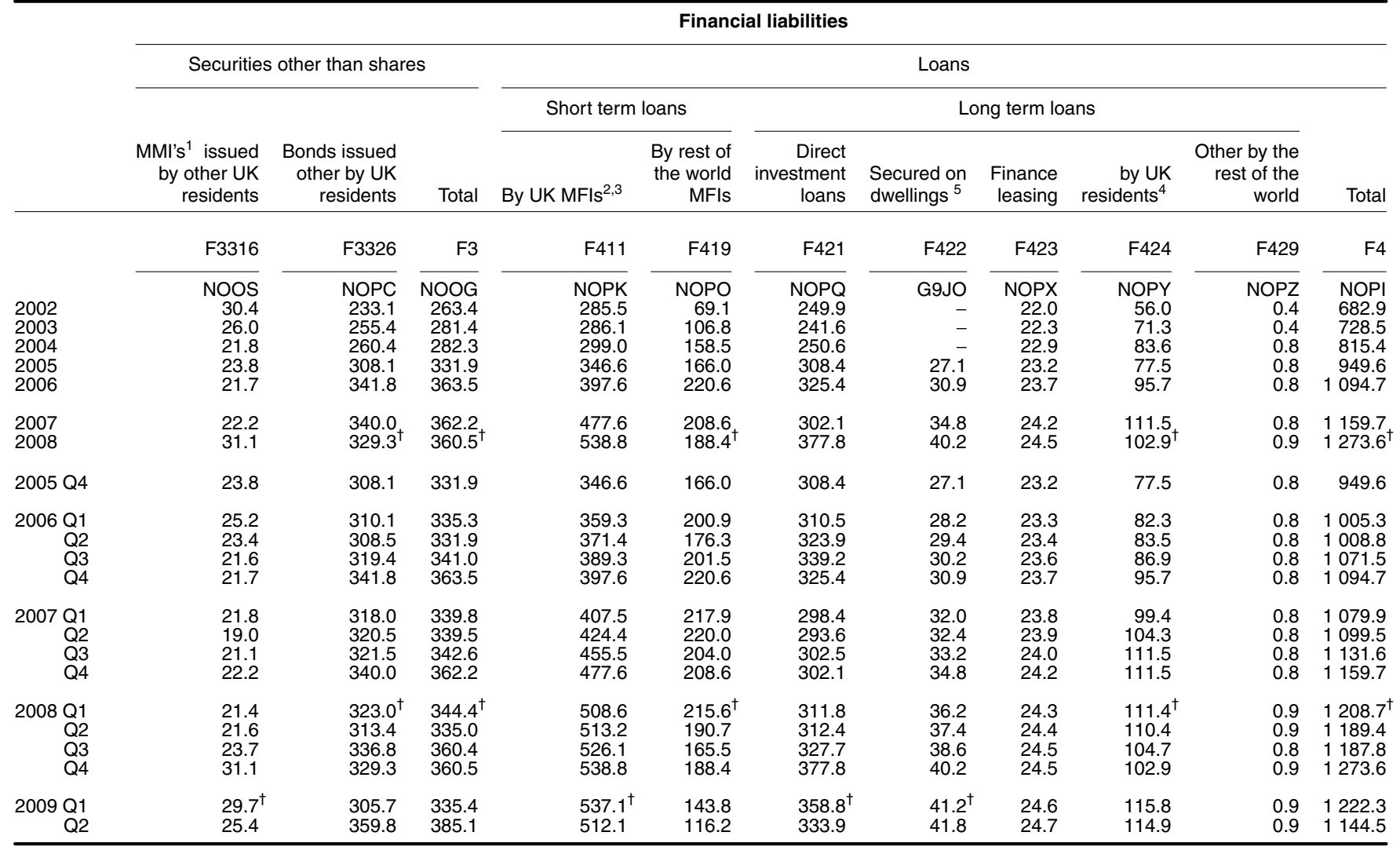

Financial liabilities - continued

Net financial
assets/liabilit-

ies

Shares and other equity

\begin{tabular}{|c|c|c|c|c|c|c|c|c|}
\hline & \multicolumn{4}{|c|}{ Shares and other equity } & \multirow[b]{3}{*}{$\begin{array}{r}\text { Other accounts } \\
\text { receivable/paya- } \\
\text { ble }\end{array}$} & \multirow[b]{3}{*}{$\begin{array}{r}\text { Total financial } \\
\text { liabilities }\end{array}$} & \multirow[b]{3}{*}{$\begin{array}{r}\text { Total financial } \\
\text { assets }\end{array}$} & \multirow[b]{3}{*}{$\begin{array}{r}\text { Net assets } \\
(+) / \text { liabilities- } \\
(-)\end{array}$} \\
\hline & \multicolumn{3}{|c|}{ Excl mutual funds' shares } & \multirow[b]{2}{*}{ Total } & & & & \\
\hline & $\begin{array}{l}\text { Quoted UK } \\
\text { companies }\end{array}$ & $\begin{array}{r}\text { Unquoted UK } \\
\text { companies }\end{array}$ & other UK equity & & & & & \\
\hline & F514 & F515 & F516 & F5 & $\mathrm{F} 7$ & $\mathrm{FL}$ & FA & BF90 \\
\hline $\begin{array}{l}2002 \\
2003 \\
2004 \\
2005 \\
2006\end{array}$ & $\begin{array}{r}\text { NOQF } \\
857.8 \\
1002.0 \\
1080.2 \\
1235.4 \\
1318.7\end{array}$ & $\begin{array}{r}\text { NOQG } \\
353.0 \\
393.2 \\
423.9 \\
515.0 \\
611.1\end{array}$ & $\begin{array}{c}\mathrm{NOQH} \\
113.1 \\
121.7 \\
132.4 \\
149.2 \\
142.3\end{array}$ & $\begin{array}{r}\text { NOQA } \\
1323.9 \\
1517.0 \\
1636.6 \\
1899.6 \\
2072.1\end{array}$ & $\begin{array}{r}\text { NOQU } \\
143.0 \\
145.4 \\
154.7 \\
159.6 \\
160.9\end{array}$ & $\begin{array}{r}\text { NONT } \\
2 \text { 413.3 } \\
2 \text { 672.2 } \\
2 \text { 888.9 } \\
3 \text { 340.7 } \\
3691.2\end{array}$ & $\begin{array}{r}\text { NNZB } \\
1198.1 \\
1324.0 \\
1420.1 \\
1581.5 \\
1699.8\end{array}$ & $\begin{array}{r}\text { NYOM } \\
-1215.1 \\
-1348.2 \\
-1468.8 \\
-1759.2 \\
-1991.3\end{array}$ \\
\hline $\begin{array}{l}2007 \\
2008\end{array}$ & $\begin{array}{r}1366.1 \\
917.9\end{array}$ & $\begin{array}{l}678.2 \\
623.0\end{array}$ & $\begin{array}{l}145.3^{\dagger} \\
148.5^{\dagger}\end{array}$ & $\begin{array}{l}2189.6 \\
1689.4^{\dagger}\end{array}$ & $\begin{array}{l}161.4^{\dagger} \\
161.5^{\dagger}\end{array}$ & $\begin{array}{l}3872.9 \\
3485.0^{\dagger}\end{array}$ & $\begin{array}{l}1915.4 \\
2219.3^{\dagger}\end{array}$ & $\begin{array}{ll}-1 & 957.5 \\
-1 & 265.7^{\dagger}\end{array}$ \\
\hline 2005 Q4 & 1235.4 & 515.0 & 149.2 & 1899.6 & 159.6 & 3340.7 & 1581.5 & -1759.2 \\
\hline $\begin{array}{r}2006 \text { Q1 } \\
\text { Q2 } \\
\text { Q3 } \\
\text { Q4 }\end{array}$ & $\begin{array}{ll}1 & 291.0 \\
1 & 253.8 \\
1 & 244.6 \\
1 & 318.7\end{array}$ & $\begin{array}{l}581.4 \\
579.2 \\
599.4 \\
611.1\end{array}$ & $\begin{array}{l}149.0 \\
150.3 \\
145.6 \\
142.3\end{array}$ & $\begin{array}{ll}2 & 021.5 \\
1 & 983.2 \\
1 & 989.5 \\
2 & 072.1\end{array}$ & $\begin{array}{l}160.4 \\
157.3 \\
159.4 \\
160.9\end{array}$ & $\begin{array}{l}3522.5 \\
3581.2 \\
3561.5 \\
3691.2\end{array}$ & $\begin{array}{ll}1 & 649.8 \\
1 & 649.5 \\
1 & 674.4 \\
1 & 699.8\end{array}$ & $\begin{array}{ll}-1 & 872.7 \\
-1 & 831.7 \\
-1 & 887.1 \\
-1 & 991.3\end{array}$ \\
\hline $\begin{array}{r}2007 \text { Q1 } \\
\text { Q2 } \\
\text { Q3 } \\
\text { Q4 }\end{array}$ & $\begin{array}{ll}1 & 371.6 \\
1 & 386.3 \\
1 & 362.0 \\
1 & 366.1\end{array}$ & $\begin{array}{l}603.3 \\
654.5 \\
667.2 \\
678.2\end{array}$ & $\begin{array}{l}138.6 \\
141.5 \\
144.6 \\
145.3\end{array}$ & $\begin{array}{l}2113.4 \\
2182.3 \\
2173.9 \\
2189.6\end{array}$ & $\begin{array}{l}166.0 \\
162.3 \\
160.5 \\
161.4\end{array}$ & $\begin{array}{l}3699.1 \\
3783.6 \\
3808.5 \\
3872.9\end{array}$ & $\begin{array}{ll}1 & 761.2 \\
1 & 832.1 \\
1 & 858.8 \\
1 & 915.4\end{array}$ & $\begin{array}{l}-1937.9 \\
-1951.5 \\
-1949.6 \\
-1957.5\end{array}$ \\
\hline $\begin{array}{r}2008 \text { Q1 } \\
\text { Q2 } \\
\text { Q3 } \\
\text { Q4 }\end{array}$ & 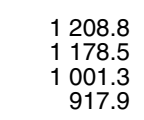 & $\begin{array}{l}679.5 \\
677.7 \\
639.8 \\
623.0\end{array}$ & $\begin{array}{l}146.7^{\dagger} \\
148.4^{\dagger} \\
148.6 \\
148.5\end{array}$ & $\begin{array}{ll}2 & 035.0 \\
2 & 004.6^{\dagger} \\
1 & 789.6 \\
1 & 689.4\end{array}$ & $\begin{array}{l}167.3^{\dagger} \\
164.5 \\
161.2 \\
161.5\end{array}$ & $\begin{array}{l}3755.5^{\dagger} \\
3693.5 \\
3499.1 \\
3485.0\end{array}$ & $\begin{array}{l}2037.8^{\dagger} \\
2035.2 \\
2059.6 \\
2219.3\end{array}$ & $\begin{array}{l}-1717.7^{\dagger} \\
-1658.3 \\
-1439.5 \\
-1265.7\end{array}$ \\
\hline $\begin{array}{r}2009 \text { Q1 } \\
\text { Q2 }\end{array}$ & $\begin{array}{l}858.1 \\
935.6\end{array}$ & $\begin{array}{l}606.4^{\dagger} \\
624.5\end{array}$ & $\begin{array}{l}148.1 \\
149.0\end{array}$ & $\begin{array}{l}1612.5 \\
1709.1\end{array}$ & $\begin{array}{l}164.4 \\
159.7\end{array}$ & $\begin{array}{l}3334.6 \\
3398.4\end{array}$ & $\begin{array}{l}2158.2 \\
2074.2\end{array}$ & $\begin{array}{ll}-1 & 176.4 \\
-1 & 324.3\end{array}$ \\
\hline
\end{tabular}

1 Money market instruments

2 All loans secured on dwellings and all finance leasing are treated as long

Sources: National Statistics, term loans

3 Monetary financial institutions

4 Other than direct investment loans, loans secured on dwellings and loans for finance leasing

5 Reflects Housing Association reclassification in line with revisions policy back to 2005 Q1. 


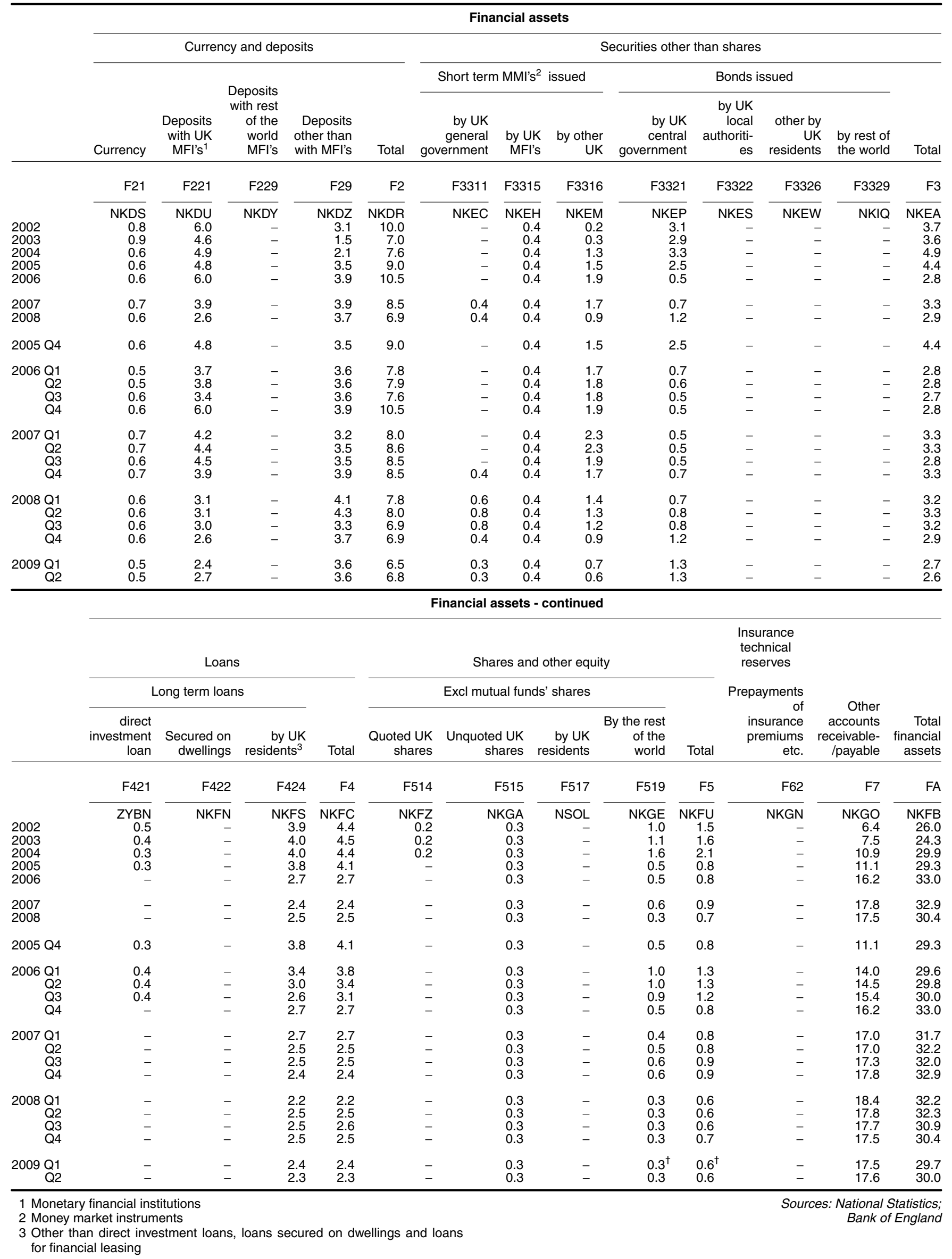




\begin{tabular}{|c|c|c|c|c|c|c|c|c|c|c|}
\hline & \multicolumn{10}{|c|}{ Financial liabilities } \\
\hline & \multicolumn{2}{|c|}{ Securities other than shares } & \multicolumn{8}{|c|}{ Loans } \\
\hline & \multirow[b]{2}{*}{$\begin{array}{r}\text { Bonds issued } \\
\text { other by UK } \\
\text { residents }\end{array}$} & \multirow[b]{2}{*}{ Total } & \multicolumn{2}{|l|}{ Short term loans } & \multirow[b]{2}{*}{$\begin{array}{r}\text { Direct } \\
\text { investment } \\
\text { loans }\end{array}$} & \multicolumn{4}{|c|}{ Long term loans } & \multirow[b]{2}{*}{ Total } \\
\hline & & & By UK MFIs ${ }^{1,2}$ & $\begin{array}{l}\text { t of the } \\
\text { Id MFIs }\end{array}$ & & Finance leasing & leasing & \multicolumn{2}{|c|}{$\begin{array}{r}\text { Other by the } \\
\text { rest of the } \\
\text { world }\end{array}$} & \\
\hline $\begin{array}{l}2002 \\
2003 \\
2004 \\
2005 \\
2006\end{array}$ & $\begin{array}{r}\text { NKIA } \\
0.9 \\
1.3 \\
5.8 \\
7.0 \\
6.3\end{array}$ & $\begin{array}{r}\text { NKHE } \\
0.9 \\
1.3 \\
5.8 \\
7.0 \\
6.3\end{array}$ & $\begin{array}{r}\text { NKII } \\
0.6 \\
0.5 \\
0.5 \\
0.5 \\
0.6\end{array}$ & $\begin{array}{r}\text { NKIM } \\
- \\
- \\
- \\
- \\
-\end{array}$ & $\begin{array}{r}\text { ZYBO } \\
- \\
- \\
- \\
\overline{-} \\
0.2\end{array}$ & & $\begin{array}{r}\text { NKIV } \\
0.4 \\
0.4 \\
0.4 \\
0.3 \\
0.3\end{array}$ & $\begin{array}{r}\text { NKIW } \\
11.3 \\
3.3 \\
4.7 \\
4.6 \\
4.4\end{array}$ & $\begin{array}{r}\text { NKIX } \\
- \\
- \\
0.4 \\
0.4 \\
0.4\end{array}$ & $\begin{array}{r}\text { NKIG } \\
12.3 \\
4.1 \\
6.0 \\
5.9 \\
5.9\end{array}$ \\
\hline $\begin{array}{l}2007 \\
2008\end{array}$ & $\begin{array}{l}6.5 \\
6.9\end{array}$ & $\begin{array}{l}6.5 \\
6.9\end{array}$ & $\begin{array}{l}0.7 \\
0.6\end{array}$ & $\begin{array}{l}- \\
-\end{array}$ & $\begin{array}{l}0.1 \\
0.1\end{array}$ & & $\begin{array}{l}0.4 \\
0.4\end{array}$ & $\begin{array}{l}4.4 \\
3.6\end{array}$ & $\begin{array}{l}0.4 \\
0.4\end{array}$ & $\begin{array}{l}6.1 \\
5.1\end{array}$ \\
\hline $\begin{array}{r}2006 \text { Q1 } \\
\text { Q2 } \\
\text { Q3 } \\
\text { Q4 }\end{array}$ & $\begin{array}{l}7.0 \\
6.8 \\
6.9 \\
6.3\end{array}$ & $\begin{array}{l}7.0 \\
6.8 \\
6.9 \\
6.3\end{array}$ & $\begin{array}{l}0.4 \\
0.4 \\
0.4 \\
0.6\end{array}$ & $\begin{array}{l}- \\
- \\
- \\
-\end{array}$ & $\begin{array}{l}0.3 \\
0.3 \\
0.3 \\
0.2\end{array}$ & & $\begin{array}{l}0.3 \\
0.3 \\
0.3 \\
0.3\end{array}$ & $\begin{array}{l}4.6 \\
4.7 \\
4.7 \\
4.4\end{array}$ & $\begin{array}{l}0.4 \\
0.4 \\
0.4 \\
0.4\end{array}$ & $\begin{array}{l}6.1 \\
6.1 \\
6.0 \\
5.9\end{array}$ \\
\hline $\begin{array}{r}2007 \text { Q1 } \\
\text { Q2 } \\
\text { Q3 } \\
\text { Q4 }\end{array}$ & $\begin{array}{l}6.3 \\
6.3 \\
6.3 \\
6.5\end{array}$ & $\begin{array}{l}6.3 \\
6.3 \\
6.3 \\
6.5\end{array}$ & $\begin{array}{l}0.5 \\
0.5 \\
0.6 \\
0.7\end{array}$ & $\begin{array}{l}- \\
- \\
- \\
-\end{array}$ & $\begin{array}{l}0.2 \\
0.2 \\
0.1 \\
0.1\end{array}$ & & $\begin{array}{l}0.3 \\
0.3 \\
0.3 \\
0.4\end{array}$ & $\begin{array}{l}4.2 \\
4.2 \\
4.1 \\
4.4\end{array}$ & $\begin{array}{l}0.4 \\
0.4 \\
0.4 \\
0.4\end{array}$ & $\begin{array}{l}5.6 \\
5.6 \\
5.6 \\
6.1\end{array}$ \\
\hline $\begin{array}{r}2008 \text { Q1 } \\
\text { Q2 } \\
\text { Q3 } \\
\text { Q4 }\end{array}$ & $\begin{array}{l}5.6 \\
5.6 \\
6.5 \\
6.9\end{array}$ & $\begin{array}{l}5.6 \\
5.6 \\
6.5 \\
6.9\end{array}$ & $\begin{array}{l}0.6 \\
0.6 \\
0.6 \\
0.6\end{array}$ & $\begin{array}{l}- \\
- \\
- \\
-\end{array}$ & $\begin{array}{l}0.1 \\
0.1 \\
0.1 \\
0.1\end{array}$ & & $\begin{array}{l}0.4 \\
0.4 \\
0.4 \\
0.4\end{array}$ & $\begin{array}{l}3.3 \\
3.3 \\
3.5 \\
3.6\end{array}$ & $\begin{array}{l}0.4 \\
0.4 \\
0.4 \\
0.4\end{array}$ & $\begin{array}{l}4.8 \\
4.8 \\
5.0 \\
5.1\end{array}$ \\
\hline \multirow[t]{5}{*}{$\begin{array}{r}2009 \text { Q1 } \\
\text { Q2 } \\
\end{array}$} & $\begin{array}{l}5.8 \\
5.7\end{array}$ & $\begin{array}{l}5.8 \\
5.7\end{array}$ & $\begin{array}{l}0.7 \\
0.6\end{array}$ & $\begin{array}{l}- \\
-\end{array}$ & $\begin{array}{l}0.1 \\
0.2\end{array}$ & & $\begin{array}{l}0.4 \\
0.4\end{array}$ & $\begin{array}{l}3.2 \\
3.3\end{array}$ & $\begin{array}{l}0.4 \\
0.4\end{array}$ & $\begin{array}{l}4.9 \\
4.9\end{array}$ \\
\hline & \multicolumn{7}{|c|}{ Financial liabilities - continued } & \multicolumn{3}{|c|}{$\begin{array}{l}\text { Net financial } \\
\text { assets/liabilit- } \\
\text { ies }\end{array}$} \\
\hline & \multicolumn{4}{|c|}{ Shares and other equity } & \multirow{3}{*}{\multicolumn{3}{|c|}{$\begin{array}{r}\text { Total financial } \\
\text { liabilities }\end{array}$}} & \multirow[b]{3}{*}{$\begin{array}{r}\text { Total financial } \\
\text { assets }\end{array}$} & \multirow{3}{*}{\multicolumn{2}{|c|}{$\begin{array}{r}\text { Net assets } \\
(+) / \text { liabilities- } \\
(-)\end{array}$}} \\
\hline & \multicolumn{3}{|c|}{ Excl mutual funds' shares } & \multirow[b]{2}{*}{ Total } & & & & & & \\
\hline & $\begin{array}{l}\text { Quoted UK } \\
\text { companies }\end{array}$ & $\begin{array}{r}\text { Unquoted UK } \\
\text { companies }\end{array}$ & Other UK equity & & & & & & & \\
\hline & F514 & F515 & F516 & F5 & & $\mathrm{F} 7$ & FL & $\mathrm{FA}$ & & BF90 \\
\hline $\begin{array}{l}2002 \\
2003 \\
2004 \\
2005 \\
2006\end{array}$ & $\begin{array}{r}\mathrm{C} 3 \mathrm{O} 8 \\
- \\
- \\
\overline{-} \\
3.0 \\
3.2\end{array}$ & $\begin{array}{r}\text { NKJE } \\
0.8 \\
0.8 \\
1.8 \\
1.8 \\
4.3\end{array}$ & $\begin{array}{r}\mathrm{H} 4 \mathrm{O} 6 \\
95.8 \\
104.4 \\
113.3 \\
129.5 \\
120.7\end{array}$ & $\begin{array}{r}\text { NKIY } \\
96.6 \\
105.3 \\
115.1 \\
134.3 \\
128.2\end{array}$ & & $\begin{array}{r}\text { NKJS } \\
13.7 \\
13.8 \\
14.7 \\
16.8 \\
15.6\end{array}$ & $\begin{array}{c}\text { NKIF } \\
123.6 \\
124.4 \\
141.5 \\
163.9 \\
156.0\end{array}$ & $\begin{array}{r}\text { NKFB } \\
26.0 \\
24.3 \\
29.9 \\
29.3 \\
33.0\end{array}$ & & $\begin{array}{r}\text { NYOP } \\
-97.6 \\
-100.2 \\
-111.6 \\
-134.6 \\
-123.0\end{array}$ \\
\hline $\begin{array}{l}2007 \\
2008\end{array}$ & $\begin{array}{l}5.6 \\
7.9\end{array}$ & $\begin{array}{l}2.7 \\
2.3\end{array}$ & $\begin{array}{l}119.1^{\dagger} \\
122.4^{\dagger}\end{array}$ & $\begin{array}{l}127.4^{\dagger} \\
132.6^{\dagger}\end{array}$ & & $\begin{array}{l}15.9^{\dagger} \\
16.1^{\dagger}\end{array}$ & $\begin{array}{l}155.9 \\
160.7^{\dagger}\end{array}$ & $\begin{array}{l}32.9 \\
30.4\end{array}$ & & $\begin{array}{l}-123.0^{\dagger} \\
-130.3^{\dagger}\end{array}$ \\
\hline 2005 Q4 & 3.0 & 1.8 & 129.5 & 134.3 & & 16.8 & 163.9 & 29.3 & & -134.6 \\
\hline $\begin{array}{r}2006 \text { Q1 } \\
\text { Q2 } \\
\text { Q3 } \\
\text { Q4 }\end{array}$ & $\begin{array}{l}3.8 \\
4.0 \\
3.4 \\
3.2\end{array}$ & $\begin{array}{l}2.7 \\
3.2 \\
3.8 \\
4.3\end{array}$ & $\begin{array}{l}129.0 \\
129.8 \\
124.4 \\
120.7\end{array}$ & $\begin{array}{l}135.5 \\
137.0 \\
131.6 \\
128.2\end{array}$ & & $\begin{array}{l}15.3 \\
15.1 \\
15.2 \\
15.6\end{array}$ & $\begin{array}{l}164.0 \\
165.1 \\
159.7 \\
156.0\end{array}$ & $\begin{array}{l}29.6 \\
29.8 \\
30.0 \\
33.0\end{array}$ & & $\begin{array}{l}-134.3 \\
-135.3 \\
-129.7 \\
-123.0\end{array}$ \\
\hline $\begin{array}{r}2007 \text { Q1 } \\
\text { Q2 } \\
\text { Q3 } \\
\text { Q4 }\end{array}$ & $\begin{array}{l}2.9 \\
5.6 \\
5.5 \\
5.6\end{array}$ & $\begin{array}{l}3.0 \\
3.0 \\
2.7 \\
2.7\end{array}$ & $\begin{array}{l}116.3 \\
116.7 \\
118.7 \\
119.1\end{array}$ & $\begin{array}{l}122.2 \\
125.3 \\
126.9 \\
127.4\end{array}$ & & $\begin{array}{l}15.5 \\
15.7 \\
15.5 \\
15.9\end{array}$ & $\begin{array}{l}149.6 \\
153.0 \\
154.2 \\
155.9\end{array}$ & $\begin{array}{l}31.7 \\
32.2 \\
32.0 \\
32.9\end{array}$ & & $\begin{array}{l}-117.9 \\
-120.8 \\
-122.3 \\
-123.0\end{array}$ \\
\hline $\begin{array}{r}2008 \text { Q1 } \\
\text { Q2 } \\
\text { Q3 } \\
\text { Q4 }\end{array}$ & $\begin{array}{l}6.7 \\
7.3 \\
7.8 \\
7.9\end{array}$ & $\begin{array}{l}2.6 \\
2.7 \\
2.2 \\
2.3\end{array}$ & $\begin{array}{l}119.4 \\
121.3^{\dagger} \\
121.9 \\
122.4\end{array}$ & $\begin{array}{l}128.8 \\
131.3^{\dagger} \\
131.9 \\
132.6\end{array}$ & & $\begin{array}{l}16.0^{\dagger} \\
15.8 \\
16.1 \\
16.1\end{array}$ & $\begin{array}{l}155.3^{\dagger} \\
157.5^{\dagger} \\
159.5 \\
160.7\end{array}$ & $\begin{array}{l}32.2 \\
32.3 \\
30.9 \\
30.4\end{array}$ & & $\begin{array}{l}-123.0^{\dagger} \\
-125.3 \\
-128.6 \\
-130.3\end{array}$ \\
\hline $\begin{array}{r}2009 \text { Q1 } \\
\text { Q2 }\end{array}$ & $\begin{array}{l}7.9 \\
7.9\end{array}$ & $\begin{array}{l}2.4 \\
2.3\end{array}$ & $\begin{array}{l}122.6 \\
123.4\end{array}$ & $\begin{array}{l}132.9 \\
133.6\end{array}$ & & $\begin{array}{l}16.3 \\
15.7\end{array}$ & $\begin{array}{l}159.8 \\
160.0\end{array}$ & $\begin{array}{l}29.7 \\
30.0\end{array}$ & & $\begin{array}{l}-130.2 \\
-130.0\end{array}$ \\
\hline
\end{tabular}

1 All loans secured on dwellings and all finance leasing are treated as long

Sources: National Statistics; loans

Bank of England

2 Monetary financial institutions

3 Other than direct investment loans, loans secured on dwellings and loans for financial leasing 


\begin{tabular}{|c|c|c|c|c|c|c|c|c|c|c|c|c|c|c|c|c|c|}
\hline & \multicolumn{17}{|c|}{ Financial assets } \\
\hline & \multicolumn{8}{|c|}{ Currency and deposits } & \multicolumn{9}{|c|}{ Securities other than shares } \\
\hline & \multirow[b]{2}{*}{$\begin{array}{r}\text { Curren- } \\
\text { cy }\end{array}$} & \multirow[b]{2}{*}{$\begin{array}{r}\text { Sterli- } \\
\text { ng Bank } \\
\text { Deposi- } \\
\text { ts }\end{array}$} & \multirow[b]{2}{*}{$\begin{array}{l}\text { Foreign } \\
\text { curren- } \\
\text { cy Bank }\end{array}$} & \multirow[b]{2}{*}{$\begin{array}{r}\text { Sterli- } \\
\text { ng } \\
\text { Buildi- } \\
\text { ng } \\
\text { societ- } \\
\text { ies }\end{array}$} & \multirow[b]{2}{*}{$\begin{array}{r}\text { Deposi- } \\
\text { ts with } \\
\text { rest of } \\
\text { the } \\
\text { world } \\
\text { MFl's }^{1}\end{array}$} & \multirow{2}{*}{\multicolumn{2}{|c|}{$\begin{array}{r}\text { Deposi- } \\
\text { ts } \\
\text { other } \\
\text { than } \\
\text { with } \\
\text { MFl's }\end{array}$}} & \multirow[b]{2}{*}{ Total } & \multicolumn{4}{|c|}{ Short term MMl's ${ }^{2}$ issued } & \multicolumn{4}{|c|}{ Bonds issued } & \multirow[b]{2}{*}{ Total } \\
\hline & & & & & & & & & $\begin{array}{r}\text { by UK } \\
\text { general } \\
\text { govern- by } \\
\text { ment }\end{array}$ & $\begin{array}{l} \\
\text { MFI's }\end{array}$ & $\begin{array}{r}\text { by } \\
\text { other } \\
\text { UK } \\
\text { reside- } \\
\text { nts }\end{array}$ & $\begin{array}{l}\text { by the } \\
\text { rest of } \\
\text { the } \\
\text { world }\end{array}$ & $\begin{array}{c}\text { by UK } \\
\text { central } \\
\text { govern- } a \\
\text { ment }\end{array}$ & $\begin{array}{c}\text { by UK } \\
\text { local } \\
\text { author- } \\
\text { ities }\end{array}$ & $\begin{array}{l}\text { Medium } \\
\text { term by resi } \\
\text { MFl's }\end{array}$ & $\begin{array}{r}\text { by } \\
\text { other by the } \\
\text { UK rest o } \\
\text { eside- the } \\
\text { nts worlc }\end{array}$ & \\
\hline & F21 & F2211 & F2212 & $\mathrm{F} 2213$ & $\mathrm{~F} 22$ & & F29 & $\mathrm{F} 2$ & F3311 F3? & 3315 & F3316 & F3319 & F3321 & F3322 & F3325 & F3326 F332s & F3 \\
\hline $\begin{array}{l}2002 \\
2003 \\
2004 \\
2005 \\
2006\end{array}$ & $\begin{array}{r}\text { NKKA } \\
3.0 \\
3.2 \\
3.4 \\
3.6 \\
3.8\end{array}$ & $\begin{array}{l}\text { NKKD } \\
145.7 \\
157.7 \\
170.1 \\
191.3 \\
214.2\end{array}$ & $\begin{array}{r}\text { NKKE } \\
23.7 \\
26.4 \\
28.0 \\
31.9 \\
30.7\end{array}$ & $\begin{array}{r}\text { NKKF } \\
2.4 \\
2.7 \\
2.4 \\
2.3 \\
2.7\end{array}$ & $\begin{array}{r}\text { NKK } \\
65 \\
121 \\
194 \\
255 \\
286\end{array}$ & $\begin{array}{l}K G \\
.1 \\
.1 \\
.2 \\
.4 \\
.9\end{array}$ & $\begin{array}{r}\text { KKH } \\
4.9 \\
5.1 \\
5.0 \\
5.1 \\
5.5\end{array}$ & $\begin{array}{l}\text { NKJZ } \\
244.8 \\
316.3 \\
403.2 \\
489.7 \\
543.9\end{array}$ & $\begin{array}{cc}\text { NKKK } & \text { NK } \\
- & \\
- & \\
- & \\
- & \\
- & \end{array}$ & $\begin{array}{r}\text { JKKP } \\
4.6 \\
5.0 \\
5.4 \\
4.8 \\
6.6\end{array}$ & $\begin{array}{r}\text { NKKU } \\
13.7 \\
13.5 \\
12.6 \\
16.4 \\
34.2\end{array}$ & $\begin{array}{r}\text { NKKV } \\
6.0 \\
9.8 \\
10.4 \\
11.4 \\
16.2\end{array}$ & $\begin{array}{r}\text { NKKX } \\
0.5 \\
0.3 \\
0.3 \\
0.2 \\
0.2\end{array}$ & $\begin{array}{r}\text { NKLA } \\
- \\
- \\
- \\
- \\
-\end{array}$ & $\begin{array}{r}\text { NKLB } \\
0.3 \\
0.9 \\
1.0 \\
1.5 \\
1.9\end{array}$ & $\begin{array}{r}\text { NKLE } \\
6.1 \\
5.2 \\
5.1 \\
1.4 \\
1.5\end{array}$ & $\begin{array}{lr}\mathrm{F} & \mathrm{NKKI} \\
.3 & 60.4 \\
.0 & 64.6 \\
.8 & 47.4 \\
.6 & 50.2 \\
.1 & 72.7\end{array}$ \\
\hline $\begin{array}{l}2007 \\
2008\end{array}$ & $\begin{array}{l}4.1^{\dagger} \\
4.3^{\dagger}\end{array}$ & $\begin{array}{l}231.3 \\
218.8\end{array}$ & $\begin{array}{l}36.2 \\
43.3\end{array}$ & $\begin{array}{l}2.9 \\
2.4\end{array}$ & $\begin{array}{l}365 \\
433\end{array}$ & & $\begin{array}{l}5.6 \\
5.5\end{array}$ & $\begin{array}{l}645.7^{\dagger} \\
707.5^{\dagger}\end{array}$ & $0 . \overline{2}$ & $\begin{array}{r}9.4^{\dagger} \\
11.7^{\dagger}\end{array}$ & $\begin{array}{l}36.8^{\dagger} \\
67.6^{\dagger}\end{array}$ & $\begin{array}{l}6.4 \\
1.3\end{array}$ & $\begin{array}{l}0.5 \\
0.7\end{array}$ & $\begin{array}{l}- \\
-\end{array}$ & $\begin{array}{l}2.5 \\
3.6\end{array}$ & $\begin{array}{l}5.2 \\
7.4\end{array}$ & $\begin{array}{lr}.34 .0^{\dagger} & 740.0^{\dagger} \\
. & 110 .\end{array}$ \\
\hline 2005 Q4 & 3.6 & 191.3 & 31.9 & 2.3 & 255 & & 5.1 & 489.7 & - & 4.8 & 16.4 & 11.4 & 0.2 & - & 1.5 & 1.4 & 50.2 \\
\hline $\begin{array}{r}2006 \text { Q1 } \\
\text { Q2 } \\
\text { Q3 } \\
\text { Q4 }\end{array}$ & $\begin{array}{l}3.7 \\
3.9 \\
3.7 \\
3.8\end{array}$ & $\begin{array}{l}196.6 \\
203.3 \\
206.1 \\
214.2\end{array}$ & $\begin{array}{l}31.8 \\
33.1 \\
31.6 \\
30.7\end{array}$ & $\begin{array}{l}2.1 \\
2.3 \\
2.6 \\
2.7\end{array}$ & $\begin{array}{l}282 \\
270 \\
291 \\
286\end{array}$ & & $\begin{array}{l}5.2 \\
5.4 \\
5.4 \\
5.5\end{array}$ & $\begin{array}{l}522.3 \\
518.3 \\
540.7 \\
543.9\end{array}$ & $\begin{array}{l}- \\
- \\
- \\
-\end{array}$ & $\begin{array}{l}5.1 \\
6.5 \\
6.4 \\
6.6\end{array}$ & $\begin{array}{l}25.5 \\
35.4 \\
29.8 \\
34.2\end{array}$ & $\begin{array}{l}13.9 \\
15.3 \\
15.1 \\
16.2\end{array}$ & $\begin{array}{l}0.2 \\
0.2 \\
0.2 \\
0.2\end{array}$ & $\begin{array}{l}- \\
- \\
- \\
-\end{array}$ & $\begin{array}{l}2.0 \\
1.7 \\
1.7 \\
1.9\end{array}$ & $\begin{array}{l}0.6 \\
1.0 \\
0.4 \\
1.5\end{array}$ & $\begin{array}{l}62.7 \\
73.0 \\
65.9 \\
72.7\end{array}$ \\
\hline $\begin{array}{r}2007 \text { Q1 } \\
\text { Q2 } \\
\text { Q3 } \\
\text { Q4 }\end{array}$ & $\begin{array}{l}3.8 \\
4.1 \\
3.9 \\
4.1\end{array}$ & $\begin{array}{l}217.0 \\
229.6 \\
225.8 \\
231.3\end{array}$ & $\begin{array}{l}34.7 \\
37.5 \\
34.4 \\
36.2\end{array}$ & $\begin{array}{l}2.6 \\
2.7 \\
2.9 \\
2.9\end{array}$ & $\begin{array}{l}317 \\
339 \\
345 \\
365\end{array}$ & & $\begin{array}{l}5.6 \\
5.5 \\
5.5 \\
5.6\end{array}$ & $\begin{array}{l}580.8 \\
619.0 \\
618.0 \\
645.7\end{array}$ & $\begin{array}{l}- \\
- \\
- \\
-\end{array}$ & $\begin{array}{r}8.9 \\
9.8 \\
10.2 \\
9.4\end{array}$ & $\begin{array}{l}32.6 \\
40.7 \\
43.5 \\
36.8\end{array}$ & $\begin{array}{r}16.0 \\
15.7 \\
10.1 \\
6.4\end{array}$ & $\begin{array}{l}0.1 \\
0.2 \\
0.4 \\
0.5\end{array}$ & $\begin{array}{l}- \\
- \\
- \\
-\end{array}$ & $\begin{array}{l}1.8 \\
1.9 \\
2.6 \\
2.5\end{array}$ & $\begin{array}{l}0.4 \\
6.9 \\
6.6 \\
5.2\end{array}$ & $\begin{array}{l}71.4 \\
87.3 \\
86.2 \\
74.0\end{array}$ \\
\hline $\begin{array}{r}2008 \text { Q1 } \\
\text { Q2 } \\
\text { Q3 } \\
\text { Q4 }\end{array}$ & $\begin{array}{l}4.1 \\
4.3^{\dagger} \\
3.9 \\
4.3\end{array}$ & $\begin{array}{l}230.8 \\
226.8 \\
220.6 \\
218.8\end{array}$ & $\begin{array}{l}38.7 \\
36.6 \\
37.5 \\
43.3\end{array}$ & $\begin{array}{l}3.2^{\dagger} \\
2.9 \\
2.5 \\
2.4\end{array}$ & $\begin{array}{l}431 \\
408 \\
410 \\
433\end{array}$ & & $\begin{array}{l}5.6 \\
5.5 \\
5.4 \\
5.5\end{array}$ & $\begin{array}{l}713.9^{\dagger} \\
684.2 \\
680.9 \\
707.5\end{array}$ & $\begin{array}{l}0 . \overline{7} \\
0.9 \\
0.2\end{array}$ & $\begin{array}{r}10.1^{\dagger} \\
10.8 \\
9.2 \\
11.7\end{array}$ & $\begin{array}{l}63.8^{\dagger} \\
60.3^{-1} \\
57.0 \\
67.6\end{array}$ & $\begin{array}{l}7.9 \\
8.7 \\
5.8 \\
1.3\end{array}$ & $\begin{array}{l}0.2 \\
0.6 \\
0.7 \\
0.7\end{array}$ & $\begin{array}{l}- \\
- \\
- \\
-\end{array}$ & $\begin{array}{l}2.6 \\
2.8 \\
3.2 \\
3.6\end{array}$ & $\begin{array}{l}6.5 \\
6.8 \\
7.0 \\
7.4\end{array}$ & $\begin{array}{ll}.8 & 107.0^{\dagger} \\
.1 & 106.8 \\
.7 & 100.5 \\
.0^{\dagger} & 110.5\end{array}$ \\
\hline \multirow[t]{5}{*}{$\begin{array}{r}2009 \text { Q1 } \\
\text { Q2 }\end{array}$} & $\begin{array}{l}4.4 \\
4.5\end{array}$ & $\begin{array}{l}219.1^{\dagger} \\
223.2\end{array}$ & $\begin{array}{l}47.0 \\
42.9\end{array}$ & $\begin{array}{l}2.9 \\
2.8\end{array}$ & $\begin{array}{l}385 \\
356\end{array}$ & & $\begin{array}{l}5.6 \\
5.4\end{array}$ & $\begin{array}{l}664.5 \\
634.7\end{array}$ & $\begin{array}{l}- \\
-\end{array}$ & $\begin{array}{l}12.1 \\
11.3\end{array}$ & $\begin{array}{l}69.6 \\
57.5\end{array}$ & $\begin{array}{l}3.6 \\
3.5\end{array}$ & $\begin{array}{l}0.3 \\
0.5\end{array}$ & $\begin{array}{l}- \\
-\end{array}$ & $\begin{array}{l}4.4^{\dagger} \\
4.4^{4}\end{array}$ & $\begin{array}{l}6.8^{\dagger} \\
6.3\end{array}$ & $\begin{array}{ll}.2 & 113.9 \\
.6 & 102.0\end{array}$ \\
\hline & \multicolumn{17}{|c|}{ Financial assets - continued } \\
\hline & \multicolumn{6}{|c|}{ Loans } & & & & ares an & ad other $\epsilon$ & equity & \multicolumn{5}{|c|}{$\begin{array}{l}\text { Insurance } \\
\text { technical } \\
\text { reserves }\end{array}$} \\
\hline & \multicolumn{5}{|c|}{ Long term loans } & \multicolumn{7}{|c|}{ Excl mutual funds' shares } & \multirow{2}{*}{\multicolumn{3}{|c|}{ 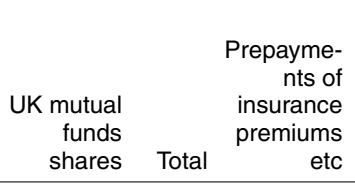 }} & \multirow[b]{2}{*}{$\begin{array}{l}\text { Other } \\
\text { accounts } \\
\text { receivab- } \\
\text { le/payabl }\end{array}$} & \multirow[b]{2}{*}{$\begin{array}{r}\text { Total } \\
\text { financial } \\
\text { assets }\end{array}$} \\
\hline & $\begin{array}{r}\text { Direct } \\
\text { investme- } \\
\text { nt loans } \\
\text { outward }\end{array}$ & $\begin{array}{r}\text { Direct } \\
\text { investme- } \\
\text { nt loans } \\
\text { inward }\end{array}$ & $\begin{array}{l}\text { Finance } \\
\text { Leasing }\end{array}$ & b) & $\begin{array}{l}\text { y UK } \\
\text { ents }\end{array}$ & Total & Quot & $\begin{array}{l}\text { ted UK } \\
\text { shares }\end{array}$ & $\begin{array}{l}\text { Unquoted } \\
\text { UK shares }\end{array}$ & $\begin{array}{l}\text { by } \\
\text { reside }\end{array}$ & UK ents the & $\begin{array}{l}\text { By the } \\
\text { rest of } \\
\text { world }\end{array}$ & & & & & \\
\hline & F4211 & F4212 & F423 & & $\mathrm{F} 424$ & $\mathrm{~F} 4$ & & F514 & F515 & & 517 & F519 & F521 & F5 & F62 & $\mathrm{F} 7$ & FA \\
\hline $\begin{array}{l}2002 \\
2003 \\
2004 \\
2005 \\
2006\end{array}$ & $\begin{array}{r}\text { NKXH } \\
110.5 \\
110.7 \\
124.5 \\
125.9 \\
129.2\end{array}$ & $\begin{array}{l}\text { NKXI } \\
52.2 \\
48.0 \\
55.5 \\
67.4 \\
72.2\end{array}$ & $\begin{array}{r}\text { F8YG } \\
2.7 \\
3.2 \\
3.6 \\
4.1 \\
4.4\end{array}$ & & $\begin{array}{r}\mathrm{KXO} \\
8.4 \\
8.2 \\
8.2 \\
8.2 \\
7.4\end{array}$ & $\begin{array}{l}\text { NKWY } \\
173.9 \\
170.1 \\
191.7 \\
205.7 \\
213.2\end{array}$ & & $\begin{array}{r}\text { NKXV } \\
8.7 \\
9.4 \\
8.8 \\
16.9 \\
32.9\end{array}$ & $\begin{array}{r}\text { NKXW } \\
39.6 \\
46.3 \\
50.0 \\
57.1 \\
60.9\end{array}$ & ) & $\begin{array}{r}\text { - } \\
- \\
- \\
- \\
-\end{array}$ & $\begin{array}{l}\text { NKYA } \\
521.3 \\
564.6 \\
549.2 \\
592.8 \\
604.8\end{array}$ & $\begin{array}{r}\text { NKYE } \\
0.3 \\
0.3 \\
0.4 \\
0.5 \\
0.6\end{array}$ & $\begin{array}{r}\text { NKXQ } \\
569.9 \\
620.7 \\
608.3 \\
667.3 \\
699.2\end{array}$ & $\begin{array}{r}\text { NKYJ } \\
12.8 \\
15.7 \\
15.5 \\
17.3 \\
16.4\end{array}$ & $\begin{array}{l}\text { NKYK } \\
110.4 \\
112.3 \\
124.0 \\
121.9 \\
121.4\end{array}$ & $\begin{array}{l}\text { NKWX } \\
1172.1 \\
1299.7 \\
1390.2 \\
1552.1 \\
1666.8\end{array}$ \\
\hline $\begin{array}{l}2007 \\
2008\end{array}$ & $\begin{array}{l}172.0 \\
253.8\end{array}$ & $\begin{array}{l}70.1 \\
87.6\end{array}$ & $\begin{array}{l}4.9 \\
5.0\end{array}$ & & $\begin{array}{l}7.6 \\
7.7\end{array}$ & $\begin{array}{l}254.6 \\
354.0\end{array}$ & & $\begin{array}{l}26.0 \\
16.8\end{array}$ & $\begin{array}{l}63.0^{\dagger} \\
59.2^{\dagger}\end{array}$ & & $\begin{array}{l}- \\
-\end{array}$ & $\begin{array}{l}684.9 \\
809.0^{\dagger}\end{array}$ & $\begin{array}{l}0.6 \\
0.3\end{array}$ & $\begin{array}{l}774.4 \\
885.3^{\dagger}\end{array}$ & $\begin{array}{l}12.9 \\
11.5\end{array}$ & $\begin{array}{l}120.9 \\
120.1\end{array}$ & $\begin{array}{l}1882.5 \\
2188.9^{\dagger}\end{array}$ \\
\hline 2005 Q4 & 125.9 & 67.4 & 4.1 & & 8.2 & 205.7 & & 16.9 & 57.1 & & - & 592.8 & 0.5 & 667.3 & 17.3 & 121.9 & 1552.1 \\
\hline $\begin{array}{r}2006 \text { Q1 } \\
\text { Q2 } \\
\text { Q3 } \\
\text { Q4 }\end{array}$ & $\begin{array}{l}133.1 \\
121.3 \\
125.4 \\
129.2\end{array}$ & $\begin{array}{l}71.1 \\
72.8 \\
73.4 \\
72.2\end{array}$ & $\begin{array}{l}4.2 \\
4.5 \\
4.4 \\
4.4\end{array}$ & & $\begin{array}{l}8.4 \\
8.4 \\
8.3 \\
7.4\end{array}$ & $\begin{array}{l}216.8 \\
206.9 \\
211.5 \\
213.2\end{array}$ & & $\begin{array}{l}20.4 \\
24.6 \\
28.3 \\
32.9\end{array}$ & $\begin{array}{l}59.6 \\
57.9 \\
57.5 \\
60.9\end{array}$ & & $\begin{array}{l}- \\
- \\
- \\
-\end{array}$ & $\begin{array}{l}598.7 \\
599.6 \\
601.8 \\
604.8\end{array}$ & $\begin{array}{l}0.6 \\
0.5 \\
0.5 \\
0.6\end{array}$ & $\begin{array}{l}679.3 \\
682.6 \\
688.1 \\
699.2\end{array}$ & $\begin{array}{l}17.2 \\
17.3 \\
16.9 \\
16.4\end{array}$ & $\begin{array}{l}121.9 \\
121.5 \\
121.3 \\
121.4\end{array}$ & $\begin{array}{ll}1 & 620.2 \\
1 & 619.7 \\
1 & 644.4 \\
1 & 666.8\end{array}$ \\
\hline $\begin{array}{r}2007 \text { Q1 } \\
\text { Q2 } \\
\text { Q3 } \\
\text { Q4 }\end{array}$ & $\begin{array}{l}139.1 \\
136.0 \\
147.2 \\
172.0\end{array}$ & $\begin{array}{l}62.7 \\
66.0 \\
69.0 \\
70.1\end{array}$ & $\begin{array}{l}4.4 \\
4.4 \\
4.7 \\
4.9\end{array}$ & & $\begin{array}{l}7.4 \\
7.5 \\
7.5 \\
7.6\end{array}$ & $\begin{array}{l}213.7 \\
213.9 \\
228.5 \\
254.6\end{array}$ & & $\begin{array}{l}33.6 \\
26.6 \\
26.0 \\
26.0\end{array}$ & $\begin{array}{l}63.4 \\
64.0 \\
62.8 \\
63.0\end{array}$ & & $\begin{array}{l}- \\
- \\
- \\
-\end{array}$ & $\begin{array}{l}629.9 \\
652.9 \\
670.6 \\
684.9\end{array}$ & $\begin{array}{l}0.6 \\
0.6 \\
0.6 \\
0.6\end{array}$ & $\begin{array}{l}727.5 \\
744.1 \\
759.9 \\
774.4\end{array}$ & $\begin{array}{l}15.1 \\
14.5 \\
13.5 \\
12.9\end{array}$ & $\begin{array}{l}121.1 \\
121.2 \\
120.7 \\
120.9\end{array}$ & $\begin{array}{l}1729.5 \\
1799.8 \\
1826.9 \\
1882.5\end{array}$ \\
\hline $\begin{array}{r}2008 \text { Q1 } \\
\text { Q2 } \\
\text { Q3 } \\
\text { Q4 }\end{array}$ & $\begin{array}{l}171.6 \\
178.3 \\
195.6 \\
253.8\end{array}$ & $\begin{array}{l}72.4 \\
75.2 \\
78.9 \\
87.6\end{array}$ & $\begin{array}{l}4.8 \\
4.8 \\
5.0 \\
5.0\end{array}$ & & $\begin{array}{l}7.6 \\
7.6 \\
7.6 \\
7.7\end{array}$ & $\begin{array}{l}256.4 \\
266.0 \\
287.1 \\
354.0\end{array}$ & & $\begin{array}{l}23.3^{\dagger} \\
21.9^{\dagger} \\
19.3 \\
16.8\end{array}$ & $\begin{array}{l}55.7 \\
54.2 \\
48.0^{\dagger} \\
59.2\end{array}$ & & $\begin{array}{l}- \\
- \\
- \\
-\end{array}$ & $\begin{array}{l}715.5^{\dagger} \\
736.8 \\
760.0 \\
809.0\end{array}$ & $\begin{array}{l}0.5 \\
0.5 \\
0.4 \\
0.3\end{array}$ & $\begin{array}{l}795.0^{\dagger} \\
813.4 \\
827.7 \\
885.3\end{array}$ & $\begin{array}{l}12.5 \\
12.3 \\
12.0 \\
11.5\end{array}$ & $\begin{array}{l}120.7 \\
120.4 \\
120.5 \\
120.1\end{array}$ & $\begin{array}{ll}2 & 005.5^{\dagger} \\
2 & 002.9 \\
2 & 028.7 \\
2 & 188.9\end{array}$ \\
\hline $\begin{array}{r}2009 \text { Q1 } \\
\text { Q2 }\end{array}$ & $\begin{array}{l}230.7^{\dagger} \\
213.8\end{array}$ & $\begin{array}{l}93.5^{\dagger} \\
86.6\end{array}$ & $\begin{array}{l}5.1^{\dagger} \\
5.1\end{array}$ & & $\begin{array}{l}7.7 \\
7.7\end{array}$ & $\begin{array}{l}337.0^{\dagger} \\
313.2\end{array}$ & & $\begin{array}{l}15.2 \\
17.2\end{array}$ & $\begin{array}{l}56.4 \\
60.0\end{array}$ & & $\begin{array}{l}- \\
-\end{array}$ & $\begin{array}{l}809.9 \\
786.1\end{array}$ & $\begin{array}{l}0.3 \\
0.4\end{array}$ & $\begin{array}{l}881.8 \\
863.6\end{array}$ & $\begin{array}{l}11.3^{\dagger} \\
11.4^{-}\end{array}$ & $\begin{array}{l}120.0 \\
119.2\end{array}$ & $\begin{array}{l}2128.5 \\
2044.2\end{array}$ \\
\hline
\end{tabular}




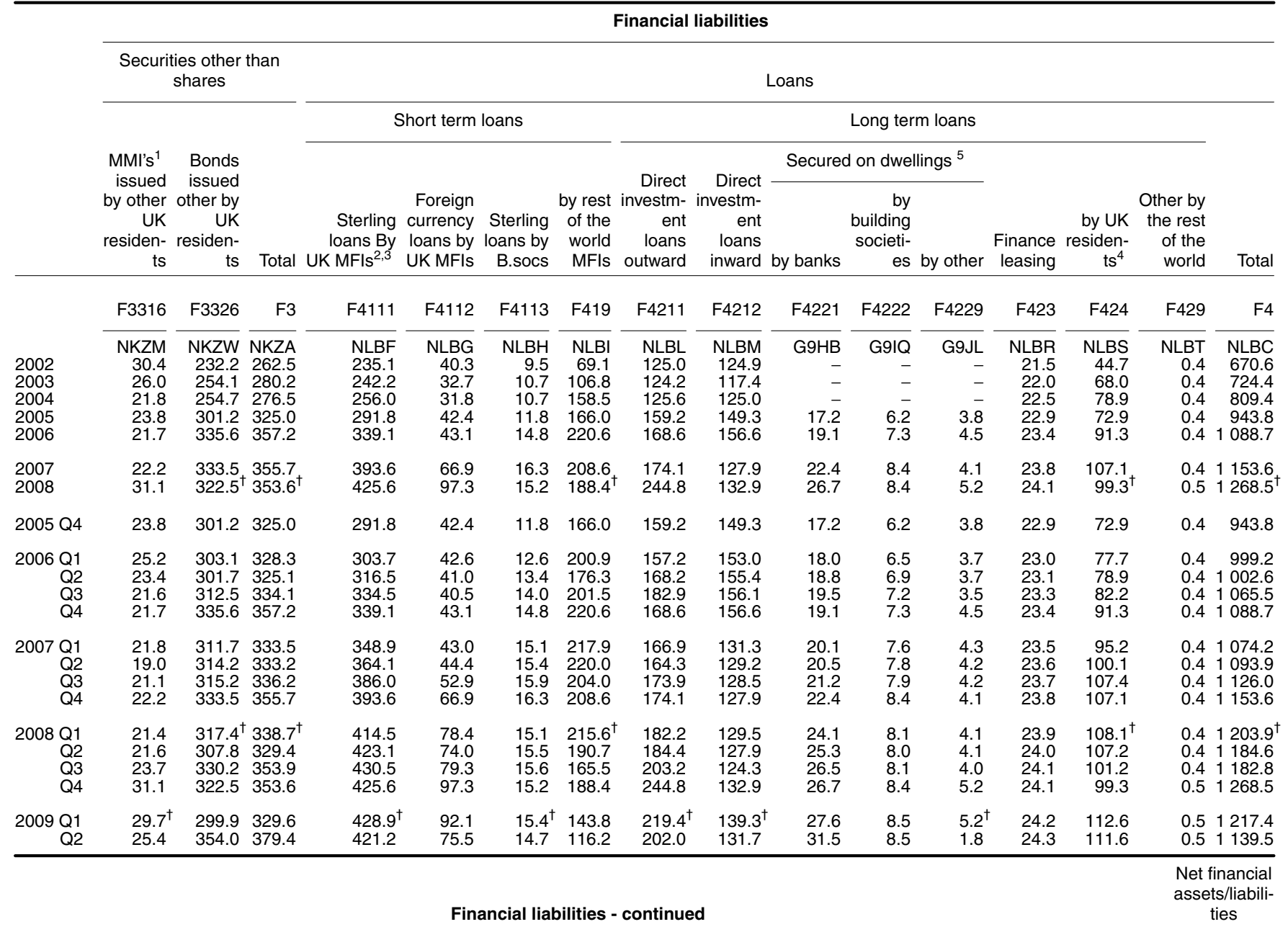

Shares and other equity

Excl mutual funds' shares

\begin{tabular}{|c|c|c|c|c|c|c|c|c|c|}
\hline & $\begin{array}{l}\text { Quoted UK } \\
\text { companies }\end{array}$ & $\begin{array}{r}\text { Unquoted UK } \\
\text { companies }\end{array}$ & Other UK equity & by UK residents & Total & $\begin{array}{r}\text { Other accounts } \\
\text { receivable/ } \\
\text { payable }\end{array}$ & $\begin{array}{r}\text { Total financial } \\
\text { liabilities }\end{array}$ & $\begin{array}{r}\text { Total financial } \\
\text { assets }\end{array}$ & $\begin{array}{r}\text { Net assets } \\
(+) / \text { /iabilitie- } \\
\text { s(-) }\end{array}$ \\
\hline & F514 & F515 & F516 & F517 & F5 & $\mathrm{F} 7$ & $\mathrm{FL}$ & FA & BF90 \\
\hline $\begin{array}{l}2002 \\
2003 \\
2004 \\
2005 \\
2006\end{array}$ & $\begin{array}{r}\text { NLBZ } \\
857.8 \\
1002.0 \\
1080.2 \\
1232.3 \\
1315.5\end{array}$ & $\begin{array}{l}\text { NLCA } \\
352.2 \\
392.4 \\
422.1 \\
513.2 \\
606.8\end{array}$ & $\begin{array}{r}\text { NLCB } \\
17.3 \\
17.3 \\
19.2 \\
19.7 \\
21.6\end{array}$ & $\begin{array}{r}\text { NSOO } \\
- \\
- \\
- \\
- \\
-\end{array}$ & $\begin{array}{r}\text { NLBU } \\
1227.2 \\
1411.7 \\
1521.5 \\
1765.3 \\
1943.9\end{array}$ & $\begin{array}{l}\text { NLCO } \\
129.3 \\
131.5 \\
140.0 \\
142.7 \\
145.3\end{array}$ & $\begin{array}{r}\text { NLBB } \\
2289.7 \\
2547.8 \\
2747.4 \\
3176.8 \\
3535.2\end{array}$ & $\begin{array}{r}\text { NKWX } \\
1172.1 \\
1299.7 \\
1390.2 \\
1552.1 \\
1666.8\end{array}$ & $\begin{array}{rr}\text { NYOT } \\
-1 & 117.6 \\
-1 & 248.1 \\
-1 & 357.2 \\
-1 & 624.7 \\
-1 & 868.4\end{array}$ \\
\hline $\begin{array}{l}2007 \\
2008\end{array}$ & $\begin{array}{r}1360.5 \\
910.0\end{array}$ & $\begin{array}{l}675.5 \\
620.7\end{array}$ & $\begin{array}{l}26.2 \\
26.1\end{array}$ & $\begin{array}{l}- \\
-\end{array}$ & $\begin{array}{l}2062.2 \\
1556.8\end{array}$ & $\begin{array}{l}145.5^{\dagger} \\
145.5^{\dagger}\end{array}$ & $\begin{array}{l}3717.0^{\dagger} \\
3324.4^{\dagger}\end{array}$ & $\begin{array}{l}1882.5 \\
2188.9^{\dagger}\end{array}$ & $\begin{array}{l}-1834.5 \\
-1135.5^{\dagger}\end{array}$ \\
\hline 2005 Q4 & 1232.3 & 513.2 & 19.7 & - & 1765.3 & 142.7 & 3176.8 & 1552.1 & -1624.7 \\
\hline $\begin{array}{r}2006 \text { Q1 } \\
\text { Q2 } \\
\text { Q3 } \\
\text { Q4 }\end{array}$ & $\begin{array}{ll}1 & 287.1 \\
1 & 249.8 \\
1 & 241.1 \\
1 & 315.5\end{array}$ & $\begin{array}{l}578.7 \\
575.9 \\
595.6 \\
606.8\end{array}$ & $\begin{array}{l}20.0 \\
20.5 \\
21.2 \\
21.6\end{array}$ & $\begin{array}{l}- \\
- \\
- \\
-\end{array}$ & $\begin{array}{l}1885.9 \\
1846.2 \\
1857.9 \\
1943.9\end{array}$ & $\begin{array}{l}145.1 \\
142.2 \\
144.3 \\
145.3\end{array}$ & $\begin{array}{l}3358.5 \\
3516.1 \\
35401.8 \\
3535.2\end{array}$ & $\begin{array}{l}1620.2 \\
1619.7 \\
1644.4 \\
1666.8\end{array}$ & $\begin{array}{ll}-1 & 738.3 \\
-1 & 696.4 \\
-1 & 757.4 \\
-1 & 868.4\end{array}$ \\
\hline $\begin{array}{r}2008 \text { Q1 } \\
\text { Q2 } \\
\text { Q3 } \\
\text { Q4 }\end{array}$ & $\begin{array}{r}1202.1 \\
1171.2 \\
993.5 \\
910.0\end{array}$ & $\begin{array}{l}676.9 \\
675.0 \\
637.6 \\
620.7\end{array}$ & $\begin{array}{l}27.3 \\
27.1 \\
26.7 \\
26.1\end{array}$ & $\begin{array}{l}- \\
- \\
- \\
-\end{array}$ & $\begin{array}{l}1906.3 \\
1873.3 \\
1657.7 \\
1556.8\end{array}$ & $\begin{array}{l}151.3^{\dagger} \\
148.7 \\
145.2 \\
145.5\end{array}$ & $\begin{array}{l}3600.2^{\dagger} \\
3536.0 \\
3339.6 \\
3324.4\end{array}$ & $\begin{array}{l}2005.5^{\dagger} \\
2002.9 \\
2028.7 \\
2188.9\end{array}$ & $\begin{array}{ll}-1 & 594.7^{\dagger} \\
-1 & 533.0 \\
-1 & 310.9 \\
-1 & 135.5\end{array}$ \\
\hline $\begin{array}{r}2009 \text { Q1 } \\
\text { Q2 }\end{array}$ & $\begin{array}{l}850.1 \\
927.7\end{array}$ & $\begin{array}{l}604.0^{\dagger} \\
622.2\end{array}$ & $\begin{array}{l}25.5 \\
25.7\end{array}$ & $\begin{array}{l}- \\
-\end{array}$ & $\begin{array}{l}1479.7^{\dagger} \\
1575.5\end{array}$ & $\begin{array}{l}148.1 \\
144.0\end{array}$ & $\begin{array}{l}3174.7 \\
3238.5\end{array}$ & $\begin{array}{l}2128.5 \\
2044.2\end{array}$ & $\begin{array}{ll}-1 & 046.2 \\
-1 & 194.3\end{array}$ \\
\hline
\end{tabular}

1 Money market instruments

2 All loans secured on dwellings and all finance leasing are treated as long term loans

3 Monetary financial institutions

4 Other than direct investment loans, loans secured on dwellings and loans for finance leasing

5 Reflects Housing Association reclassification in line with revisions policy back to 2005 Q1. 


\begin{tabular}{|c|c|c|c|c|c|c|c|c|c|c|c|c|c|c|c|c|}
\hline & \multicolumn{16}{|c|}{ Financial assets } \\
\hline & \multicolumn{5}{|c|}{ Currency and deposits } & \multicolumn{11}{|c|}{ Securities other than shares } \\
\hline & \multirow[b]{2}{*}{ Currency } & \multirow{2}{*}{$\begin{array}{c}\text { Deposits } \\
\text { with UK } \\
\text { MFI's }^{1}\end{array}$} & \multirow{2}{*}{$\begin{array}{r}\text { Deposits } \\
\text { with } \\
\text { rest of } \\
\text { the } \\
\text { world } \\
\text { MFl's }\end{array}$} & \multirow[b]{2}{*}{$\begin{array}{r}\text { Deposits } \\
\text { other } \\
\text { than } \\
\text { with } \\
\text { MFl's }\end{array}$} & \multirow[b]{2}{*}{ Total } & \multicolumn{5}{|c|}{ Short term MMl's ${ }^{2}$ issued } & \multicolumn{5}{|c|}{ Bonds issued } & \multirow[b]{2}{*}{ Total } \\
\hline & & & & & & $\begin{array}{r}\text { by UK } \\
\text { general } \\
\text { governm- } \\
\text { ent }\end{array}$ & $\begin{array}{l}\text { UK local } \\
\text { authori- } \\
\text { ty bills }\end{array}$ & $\begin{array}{c}\text { by UK } \\
\text { MFI's }\end{array}$ & $\begin{array}{r}\text { by other } \\
\text { UK } \\
\text { residen- } \\
\text { ts }\end{array}$ & $\begin{array}{l}\text { by the } \\
\text { rest of } \\
\text { the } \\
\text { world }\end{array}$ & $\begin{array}{r}\text { by UK } \\
\text { central } \\
\text { governm- } \\
\text { ent }\end{array}$ & $\begin{array}{r}\text { by UK } \\
\text { local } \\
\text { authori- } \\
\text { ties }\end{array}$ & $\begin{array}{l}\text { Medium } \\
\text { term by } \\
\text { MFl's }\end{array}$ & $\begin{array}{r}\text { Other by } \\
\text { UK } \\
\text { residen- } \\
\text { ts }\end{array}$ & $\begin{array}{r}\text { by the } \\
\text { rest of } \\
\text { the } \\
\text { world }\end{array}$ & \\
\hline & $\mathrm{F} 21$ & F221 & F229 & F29 & $\mathrm{F} 2$ & F3311 & F3312 & F3315 & F3316 & F3319 & F3321 & F3322 & F3325 & F3326 & F3329 & F3 \\
\hline $\begin{array}{l}2002 \\
2003 \\
2004 \\
2005 \\
2006\end{array}$ & $\begin{array}{r}\text { NLJE } \\
7.4 \\
8.3 \\
11.3 \\
10.2 \\
10.1\end{array}$ & $\begin{array}{r}\text { NLJG } \\
790.9 \\
1041.7 \\
1180.6 \\
1446.1 \\
1833.6\end{array}$ & $\begin{array}{rr} & \text { NLJK } \\
1 & 099.7 \\
1 & 233.1 \\
1 & 357.0 \\
1 & 740.4 \\
1 & 838.1\end{array}$ & $\begin{array}{r}\text { NLJL } \\
1.9 \\
0.8 \\
2.2 \\
3.6 \\
1.5\end{array}$ & $\begin{array}{rl} & \text { NLJD } \\
1 & 899.8 \\
2 & 283.9 \\
2 & 551.1 \\
3 & 200.4 \\
3 & 683.3\end{array}$ & $\begin{array}{r}\text { NLJO } \\
21.0 \\
21.9 \\
21.1 \\
18.2 \\
15.7\end{array}$ & $\begin{array}{r}\text { NLJS } \\
- \\
- \\
- \\
- \\
-\end{array}$ & $\begin{array}{l}\text { NLJT } \\
151.1 \\
140.0 \\
139.8 \\
144.1 \\
150.4\end{array}$ & $\begin{array}{r}\text { NLJY } \\
5.3 \\
7.3 \\
5.1 \\
9.4 \\
10.3\end{array}$ & $\begin{array}{r}\text { NLJZ } \\
41.6 \\
52.1 \\
47.6 \\
50.6 \\
56.3\end{array}$ & $\begin{array}{l}\text { NLKB } \\
210.5 \\
227.3 \\
254.3 \\
273.1 \\
296.9\end{array}$ & $\begin{array}{r}\text { NLKE } \\
0.5 \\
0.5 \\
0.4 \\
0.4 \\
0.5\end{array}$ & $\begin{array}{r}\text { NLKF } \\
37.1 \\
52.4 \\
62.8 \\
78.5 \\
89.0\end{array}$ & $\begin{array}{c}\text { NLKI } \\
278.0 \\
309.9 \\
345.0 \\
409.8 \\
464.4\end{array}$ & $\begin{array}{l}\text { NLKJ } \\
484.4 \\
496.2 \\
573.7 \\
677.4 \\
763.6\end{array}$ & $\begin{array}{r}\text { NLJM } \\
1229.6 \\
1307.6 \\
1449.8 \\
1661.6 \\
1847.1\end{array}$ \\
\hline $\begin{array}{l}2007 \\
2008\end{array}$ & $\begin{array}{l}8.7 \\
8.1\end{array}$ & $\begin{array}{l}1533.1 \\
1880.5^{\dagger}\end{array}$ & $\begin{array}{l}2330.8 \\
2528.7^{\dagger}\end{array}$ & $\begin{array}{r}3.8 \\
11.5\end{array}$ & $\begin{array}{l}3 \text { 876.3 } \\
4428.7^{\dagger}\end{array}$ & $\begin{array}{r}10.3^{\dagger} \\
9.8^{\dagger}\end{array}$ & $\begin{array}{l}- \\
-\end{array}$ & $\begin{array}{l}148.1^{\dagger} \\
136.0^{\dagger}\end{array}$ & $\begin{array}{l}9.4 \\
5.4\end{array}$ & $\begin{array}{l}64.5^{\dagger} \\
66.8^{\dagger}\end{array}$ & $\begin{array}{l}319.1 \\
406.0^{\dagger}\end{array}$ & $\begin{array}{l}0.3 \\
0.2\end{array}$ & $\begin{array}{l}115.9 \\
153.8\end{array}$ & $\begin{array}{l}471.3^{\dagger} \\
446.0^{\dagger}\end{array}$ & $\begin{array}{r}866.2 \\
1120.2^{\dagger}\end{array}$ & 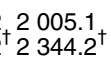 \\
\hline 2005 Q4 & 10.2 & 1446.1 & 1740.4 & 3.6 & 3200.4 & 18.2 & - & 144.1 & 9.4 & 50.6 & 273.1 & 0.4 & 78.5 & 409.8 & 677.4 & 1661.6 \\
\hline $\begin{array}{r}2006 \text { Q1 } \\
\text { Q2 } \\
\text { Q3 } \\
\text { Q4 }\end{array}$ & $\begin{array}{r}8.7 \\
8.8 \\
9.0 \\
10.1\end{array}$ & $\begin{array}{l}1620.9 \\
1671.2 \\
1729.5 \\
1833.6\end{array}$ & $\begin{array}{l}1954.7 \\
1883.2 \\
1876.9 \\
1838.1\end{array}$ & $\begin{array}{l}1.5 \\
1.7 \\
0.8 \\
1.5\end{array}$ & $\begin{array}{l}3585.9 \\
3564.9 \\
3516.3 \\
3683.3\end{array}$ & $\begin{array}{l}15.7 \\
16.6 \\
15.5 \\
15.7\end{array}$ & $\begin{array}{l}- \\
- \\
- \\
-\end{array}$ & $\begin{array}{l}152.1 \\
152.6 \\
155.1 \\
150.4\end{array}$ & $\begin{array}{r}7.5 \\
8.8 \\
11.2 \\
10.3\end{array}$ & $\begin{array}{l}56.4 \\
59.9 \\
56.2 \\
56.3\end{array}$ & $\begin{array}{l}295.5 \\
306.6 \\
309.0 \\
296.9\end{array}$ & $\begin{array}{l}0.6 \\
0.5 \\
0.5 \\
0.5\end{array}$ & $\begin{array}{l}87.6 \\
82.0 \\
84.9 \\
89.0\end{array}$ & $\begin{array}{l}427.5 \\
428.6 \\
443.0 \\
464.4\end{array}$ & $\begin{array}{l}703.1 \\
703.9 \\
709.9 \\
763.6\end{array}$ & $\begin{array}{l}1745.9 \\
1759.6 \\
1785.3 \\
1847.1\end{array}$ \\
\hline $\begin{array}{r}2007 \text { Q1 } \\
\text { Q2 } \\
\text { Q3 } \\
\text { Q4 }\end{array}$ & $\begin{array}{l}9.3 \\
9.3 \\
9.2 \\
8.7\end{array}$ & $\begin{array}{ll}1 & 967.7 \\
1 & 957.1 \\
1 & 597.1 \\
1 & 533.1\end{array}$ & $\begin{array}{l}2081.5 \\
2079.8 \\
2177.8 \\
2330.8\end{array}$ & $\begin{array}{l}1.2 \\
2.8 \\
3.5 \\
3.8\end{array}$ & $\begin{array}{l}4059.7 \\
4049.0 \\
3787.7 \\
3876.3\end{array}$ & $\begin{array}{l}11.4 \\
11.8 \\
10.2 \\
10.3\end{array}$ & $\begin{array}{l}- \\
- \\
- \\
-\end{array}$ & $\begin{array}{l}159.9 \\
148.8 \\
156.7 \\
148.1\end{array}$ & $\begin{array}{r}10.6 \\
10.3 \\
12.1 \\
9.4\end{array}$ & $\begin{array}{l}65.2 \\
67.0 \\
62.9 \\
64.5\end{array}$ & $\begin{array}{l}303.1 \\
288.0 \\
306.6 \\
319.1\end{array}$ & $\begin{array}{l}0.5 \\
0.4 \\
0.4 \\
0.3\end{array}$ & $\begin{array}{r}94.6 \\
105.3 \\
113.1 \\
115.9\end{array}$ & $\begin{array}{l}472.7 \\
466.4 \\
463.2 \\
471.3\end{array}$ & $\begin{array}{l}801.7 \\
825.3 \\
828.9 \\
866.2\end{array}$ & $\begin{array}{l}1919.6 \\
1923.3 \\
1954.0 \\
2005.1\end{array}$ \\
\hline $\begin{array}{r}2008 \text { Q1 } \\
\text { Q2 } \\
\text { Q3 } \\
\text { Q4 }\end{array}$ & $\begin{array}{l}7.0 \\
6.4 \\
6.7 \\
8.1\end{array}$ & $\begin{array}{ll}1 & 706.1 \\
1 & 583.8 \\
1 & 770.8^{\dagger} \\
1 & 880.5\end{array}$ & $\begin{array}{l}2586.5^{\dagger} \\
2281.7 \\
2412.8 \\
2528.7\end{array}$ & $\begin{array}{r}5.1 \\
11.8 \\
7.6 \\
11.5\end{array}$ & $\begin{array}{ll}4 & 304.7^{\dagger} \\
3 & 883.7 \\
4 & 197.8 \\
4 & 428.7\end{array}$ & $\begin{array}{l}8.9 \\
7.7^{\dagger} \\
9.3 \\
9.8\end{array}$ & & $\begin{array}{l}150.7^{\dagger} \\
143.4 \\
136.7 \\
136.0\end{array}$ & $\begin{array}{l}8.3^{\dagger} \\
8.2 \\
8.9 \\
5.4\end{array}$ & $\begin{array}{l}63.8 \\
68.1 \\
70.1 \\
66.8^{\dagger}\end{array}$ & $\begin{array}{l}325.5^{\dagger} \\
321.5 \\
327.9 \\
406.0\end{array}$ & $\begin{array}{l}0.3 \\
0.3 \\
0.3 \\
0.2\end{array}$ & $\begin{array}{l}115.8 \\
119.4 \\
121.0 \\
153.8\end{array}$ & $\begin{array}{l}440.9^{\dagger} \\
450.5 \\
452.9 \\
446.0\end{array}$ & $\begin{array}{r}901.7^{\dagger} \\
905.0 \\
971.2 \\
1120.2\end{array}$ & $\begin{array}{l}{ }^{\dagger} 2015.8^{\dagger} \\
2024.2 \\
2098.2 \\
2344.2\end{array}$ \\
\hline $\begin{array}{r}2009 \text { Q1 } \\
\text { Q2 }\end{array}$ & $\begin{array}{l}6.5 \\
7.5\end{array}$ & $\begin{array}{l}1843.5 \\
1845.5\end{array}$ & $\begin{array}{l}2430.6 \\
2210.0\end{array}$ & $\begin{array}{l}24.3 \\
14.5\end{array}$ & $\begin{array}{l}4304.9 \\
4077.5\end{array}$ & $\begin{array}{l}18.2 \\
23.4\end{array}$ & $\begin{array}{l}- \\
-\end{array}$ & $\begin{array}{l}124.5 \\
112.1\end{array}$ & $\begin{array}{l}4.7 \\
4.9\end{array}$ & $\begin{array}{l}67.9 \\
68.2\end{array}$ & $\begin{array}{l}415.2 \\
469.5\end{array}$ & $\begin{array}{l}0.1^{\dagger} \\
0.2^{\dagger}\end{array}$ & $\begin{array}{l}169.5^{\dagger} \\
170.4\end{array}$ & $\begin{array}{l}403.7 \\
458.5\end{array}$ & $\begin{array}{ll}1 & 160.7 \\
1 & 081.7\end{array}$ & $\begin{array}{l}2364.5 \\
2389.0\end{array}$ \\
\hline
\end{tabular}

Financial assets - continued

\begin{tabular}{|c|}
\hline Loans \\
\hline
\end{tabular}

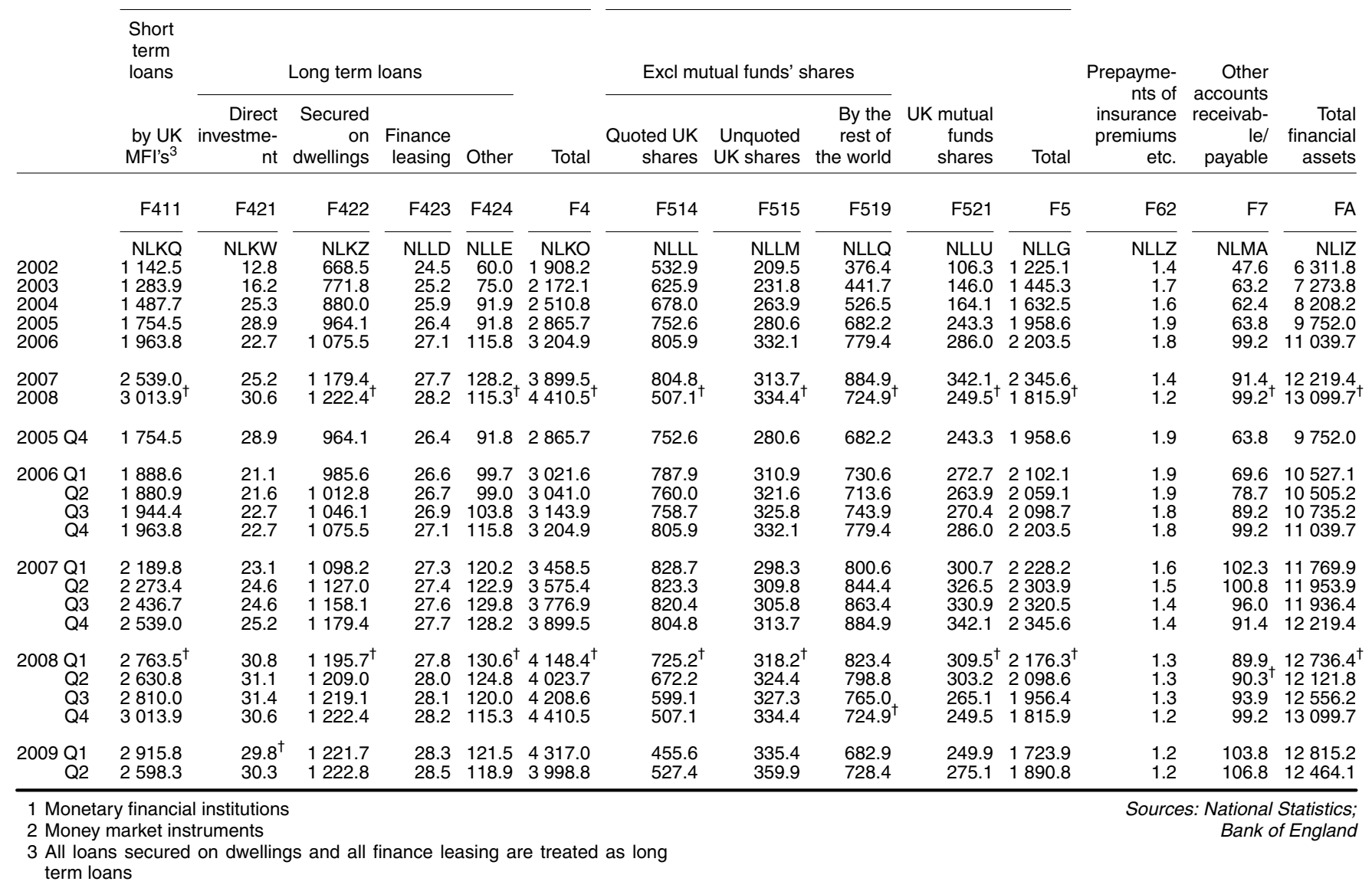




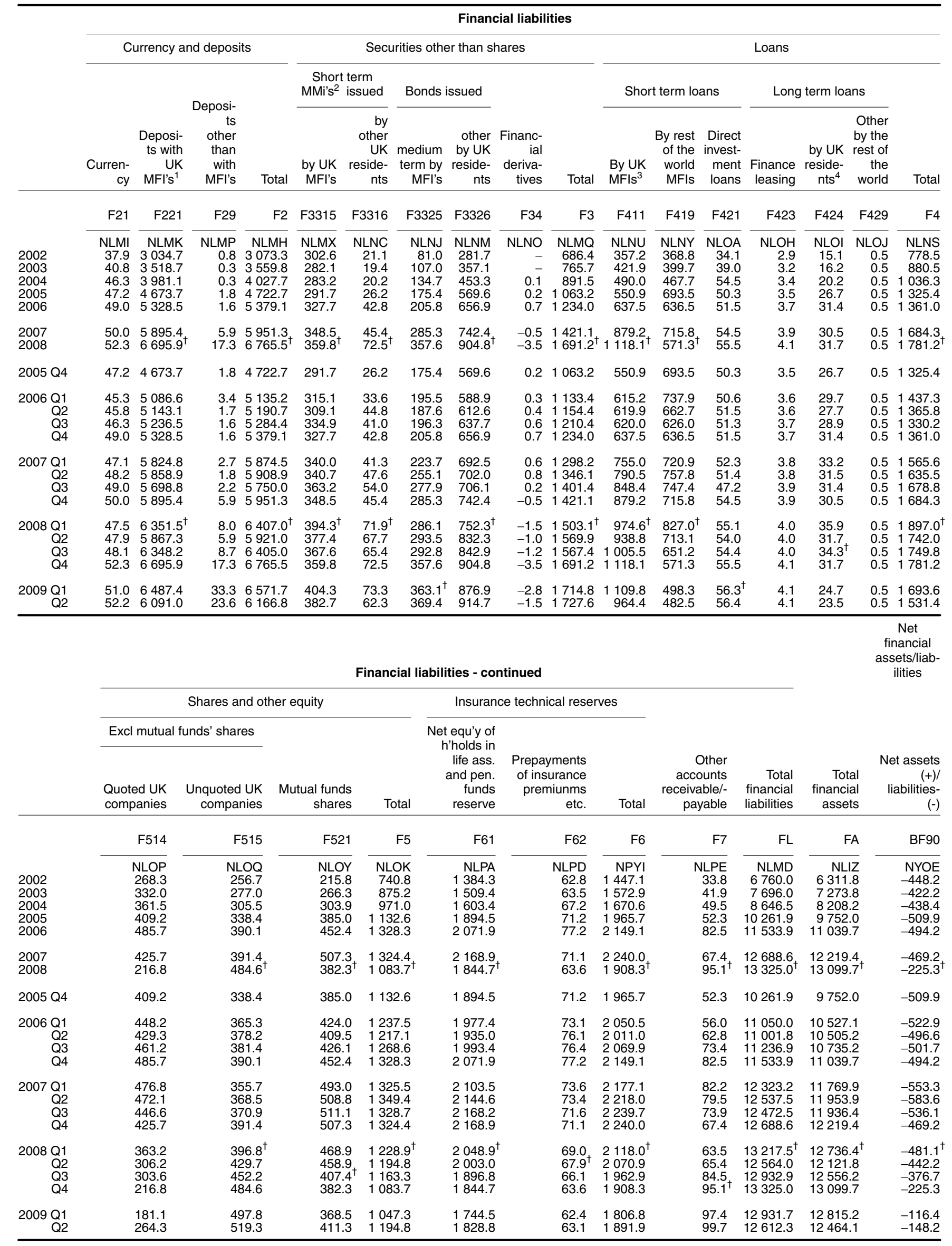

1 Monetary financial institutions

2 Money market instruments

Sources: National Statistics;

3 All loans secured on dwellings and all finance leasing are treated as long

Bank of England

4 Other than direct investment loans, loans secured on dwellings and loans

for finance leasing 


\begin{tabular}{|c|c|c|c|c|c|c|c|c|c|c|c|c|c|c|c|c|c|}
\hline & \multicolumn{17}{|c|}{ Financial assets(MFI) } \\
\hline & \multicolumn{5}{|c|}{ Currency and deposits } & \multicolumn{12}{|c|}{ Securities other than shares } \\
\hline & \multirow[b]{2}{*}{$\begin{array}{r}\text { Curren- } \\
\text { cy }\end{array}$} & \multirow[b]{2}{*}{$\begin{array}{c}\text { Deposi- } \\
\text { ts with } \\
\text { UK } \\
\text { MFI's }^{1}\end{array}$} & \multirow[b]{2}{*}{$\begin{array}{r}\text { Deposi- } \\
\text { ts with } \\
\text { rest of } \\
\text { the } \\
\text { world } \\
\text { MFl's }\end{array}$} & \multirow[b]{2}{*}{$\begin{array}{r}\text { Deposi- } \\
\text { ts } \\
\text { other } \\
\text { than } \\
\text { with } \\
\text { MFl's }\end{array}$} & \multirow[b]{2}{*}{ Total } & \multicolumn{5}{|c|}{ Short term MMl's ${ }^{2}$ issued } & \multicolumn{6}{|c|}{ Bonds issued } & \multirow[b]{2}{*}{ Total } \\
\hline & & & & & & $\begin{array}{r}\text { by UK } \\
\text { general } \\
\text { govern- } \\
\text { ment }\end{array}$ & $\begin{array}{r}\text { UK } \\
\text { local } \\
\text { author- } \\
\text { ity } \\
\text { bills }\end{array}$ & $\begin{array}{r}\text { by UK } \\
\text { MFI's }\end{array}$ & $\begin{array}{r}\text { by } \\
\text { other } \\
\text { UK } \\
\text { reside- } \\
\text { nts }\end{array}$ & $\begin{array}{r}\text { by the } \\
\text { rest of } \\
\text { the } \\
\text { world }\end{array}$ & $\begin{array}{r}\text { by UK } \\
\text { central } \\
\text { govern- } \\
\text { ment }\end{array}$ & $\begin{array}{r}\text { by UK } \\
\text { local } \\
\text { author- } \\
\text { ities }\end{array}$ & $\begin{array}{r}\text { Medium } \\
\text { term by } \\
\text { MFl's }\end{array}$ & $\begin{array}{r}\text { Other } \\
\text { by UK } \\
\text { reside- } \\
\text { nts }\end{array}$ & $\begin{array}{l}\text { by the } \\
\text { rest of } \\
\text { the } \\
\text { world }\end{array}$ & $\begin{array}{r}\text { Financ- } \\
\text { ial } \\
\text { deriva- } \\
\text { tives }\end{array}$ & \\
\hline & $\mathrm{F} 21$ & F221 & F229 & $\mathrm{F} 29$ & $\mathrm{~F} 2$ & F3311 & F3312 & F3315 & F3316 & F3319 & F3321 & F3322 & F3325 & F3326 & F3329 & F34 & F3 \\
\hline $\begin{array}{l}2002 \\
2003 \\
2004 \\
2005 \\
2006\end{array}$ & $\begin{array}{r}\text { NNSY } \\
7.3 \\
8.2 \\
11.3 \\
10.2 \\
10.0\end{array}$ & $\begin{array}{r}\text { NNTA } \\
509.1 \\
736.4 \\
831.9 \\
1000.5 \\
1282.2\end{array}$ & $\begin{array}{r}\text { NNTE } \\
843.0 \\
921.4 \\
1006.9 \\
1199.7 \\
1286.0\end{array}$ & $\begin{array}{r}\text { NNTF } \\
- \\
- \\
- \\
- \\
-\end{array}$ & $\begin{array}{rr} & \text { NNSX } \\
1 & 359.5 \\
1 & 666.1 \\
1 & 850.1 \\
2 & 210.4 \\
2 & 578.1\end{array}$ & $\begin{array}{r}\text { NNTI } \\
19.3 \\
18.4 \\
16.1 \\
15.8 \\
12.1\end{array}$ & $\begin{array}{r}\text { NNTM } \\
- \\
- \\
- \\
- \\
-\end{array}$ & $\begin{array}{r}\text { NNTN } \\
102.5 \\
89.8 \\
90.9 \\
96.1 \\
98.4\end{array}$ & $\begin{array}{r}\text { NNTS } \\
1.1 \\
3.2 \\
1.9 \\
2.9 \\
5.0\end{array}$ & $\begin{array}{r}\text { NNTT } \\
34.6 \\
42.8 \\
37.0 \\
39.6 \\
42.8\end{array}$ & $\begin{array}{r}\text { NNTV } \\
-1.0 \\
-6.3 \\
-1.4 \\
-2.8 \\
-7.7\end{array}$ & $\begin{array}{r}\text { NNTY } \\
- \\
- \\
- \\
- \\
-\end{array}$ & $\begin{array}{r}\text { NNTZ } \\
18.5 \\
21.1 \\
23.4 \\
25.2 \\
24.5\end{array}$ & $\begin{array}{r}\text { NNUC } \\
50.9 \\
65.9 \\
90.0 \\
133.6 \\
189.4\end{array}$ & $\begin{array}{r}\text { NNUD } \\
336.3 \\
327.3 \\
359.7 \\
413.7 \\
479.3\end{array}$ & $\begin{array}{r}\text { NNUE } \\
- \\
- \\
- \\
- \\
-\end{array}$ & $\begin{array}{r}\text { NNTG } \\
562.2 \\
562.2 \\
617.7 \\
724.1 \\
843.8\end{array}$ \\
\hline 2005 Q4 & 10.2 & 1000.5 & 1199.7 & - & 2210.4 & 15.8 & - & 96.1 & 2.9 & 39.6 & -2.8 & - & 25.2 & 133.6 & 413.7 & - & 724.1 \\
\hline $\begin{array}{r}2006 \text { Q1 } \\
\text { Q2 } \\
\text { Q3 } \\
\text { Q4 }\end{array}$ & $\begin{array}{r}8.7 \\
8.7 \\
8.9 \\
10.0\end{array}$ & $\begin{array}{ll}1 & 131.0 \\
1 & 176.6 \\
1 & 195.8 \\
1 & 282.2\end{array}$ & $\begin{array}{ll}1 & 329.5 \\
1 & 309.2 \\
1 & 337.8 \\
1 & 286.0\end{array}$ & $\begin{array}{l}- \\
- \\
- \\
-\end{array}$ & $\begin{array}{l}2469.3 \\
24494.6 \\
2542.6 \\
2578.1\end{array}$ & $\begin{array}{l}12.0 \\
11.6 \\
11.9 \\
12.1\end{array}$ & $\begin{array}{l}- \\
- \\
- \\
-\end{array}$ & $\begin{array}{r}103.1 \\
98.5 \\
100.9 \\
98.4\end{array}$ & $\begin{array}{l}3.4 \\
2.4 \\
3.6 \\
5.0\end{array}$ & $\begin{array}{l}44.3 \\
45.9 \\
43.6 \\
42.8\end{array}$ & $\begin{array}{l}-4.4 \\
-0.2 \\
-2.7 \\
-7.7\end{array}$ & $\begin{array}{l}- \\
- \\
- \\
-\end{array}$ & $\begin{array}{l}26.5 \\
23.6 \\
23.7 \\
24.5\end{array}$ & $\begin{array}{l}160.4 \\
162.9 \\
173.0 \\
189.4\end{array}$ & $\begin{array}{l}430.4 \\
438.7 \\
441.5 \\
479.3\end{array}$ & $\begin{array}{l}- \\
- \\
- \\
-\end{array}$ & $\begin{array}{l}775.8 \\
783.5 \\
795.5 \\
843.8\end{array}$ \\
\hline $\begin{array}{r}2007 \text { Q1 } \\
\text { Q2 } \\
\text { Q3 } \\
\text { Q4 }\end{array}$ & $\begin{array}{l}9.2 \\
9.3 \\
9.2 \\
8.6\end{array}$ & $\begin{array}{r}1380.1 \\
1318.1 \\
937.3 \\
842.6\end{array}$ & $\begin{array}{l}1460.9 \\
1483.7 \\
1578.9 \\
1714.1\end{array}$ & $\begin{array}{l}- \\
- \\
- \\
-\end{array}$ & $\begin{array}{l}2850.2 \\
2811.1 \\
2525.4 \\
2565.3\end{array}$ & $\begin{array}{l}8.2 \\
8.0 \\
4.7 \\
5.6\end{array}$ & $\begin{array}{l}- \\
- \\
- \\
-\end{array}$ & $\begin{array}{r}104.6 \\
95.1 \\
95.1 \\
88.7\end{array}$ & $\begin{array}{l}5.4 \\
2.9 \\
2.5 \\
1.3\end{array}$ & $\begin{array}{l}51.7 \\
51.8 \\
50.4 \\
53.8\end{array}$ & $\begin{array}{r}-16.5 \\
-10.1 \\
-8.1 \\
-5.5\end{array}$ & $\begin{array}{l}- \\
- \\
- \\
-\end{array}$ & $\begin{array}{l}25.8 \\
27.0 \\
24.0 \\
25.2\end{array}$ & $\begin{array}{l}199.6 \\
203.5 \\
206.4 \\
209.4\end{array}$ & $\begin{array}{l}510.0 \\
531.4 \\
537.1 \\
555.2\end{array}$ & $\begin{array}{l}- \\
- \\
- \\
-\end{array}$ & $\begin{array}{l}888.9 \\
909.6 \\
912.1 \\
933.6\end{array}$ \\
\hline $\begin{array}{r}2008 \text { Q1 } \\
\text { Q2 } \\
\text { Q3 } \\
\text { Q4 }\end{array}$ & $\begin{array}{l}6.9 \\
6.3 \\
6.6 \\
8.0\end{array}$ & $\begin{array}{l}929.9 \\
802.5 \\
943.1 \\
924.5\end{array}$ & 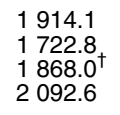 & $\begin{array}{l}- \\
- \\
- \\
-\end{array}$ & $\begin{array}{l}2851.0 \\
2531.7^{\dagger} \\
2817.8^{\dagger} \\
3025.2\end{array}$ & $\begin{array}{l}4.4 \\
4.4 \\
2.0 \\
3.1\end{array}$ & $\begin{array}{l}- \\
- \\
- \\
-\end{array}$ & $\begin{array}{l}90.0 \\
79.8 \\
77.5 \\
72.5\end{array}$ & $\begin{array}{l}0.9 \\
0.9 \\
0.8 \\
0.5\end{array}$ & $\begin{array}{l}52.1 \\
56.9 \\
60.5 \\
57.1\end{array}$ & $\begin{array}{r}-2.8 \\
-2.9 \\
3.2 \\
25.7\end{array}$ & $\begin{array}{l}- \\
- \\
- \\
-\end{array}$ & $\begin{array}{l}25.1 \\
25.8 \\
26.7 \\
43.0\end{array}$ & $\begin{array}{l}182.4^{\dagger} \\
187.3 \\
191.5 \\
183.7\end{array}$ & $\begin{array}{l}555.3 \\
517.5^{\dagger} \\
530.1 \\
568.7\end{array}$ & $\begin{array}{l}- \\
- \\
- \\
-\end{array}$ & $\begin{array}{l}907.4^{\dagger} \\
869.7 \\
892.3 \\
954.3\end{array}$ \\
\hline $\begin{array}{r}2009 \text { Q1 } \\
\text { Q2 }\end{array}$ & $\begin{array}{l}6.4 \\
7.4\end{array}$ & $\begin{array}{l}846.0 \\
890.7\end{array}$ & $\begin{array}{l}2039.4 \\
1813.9\end{array}$ & $\begin{array}{l}- \\
-\end{array}$ & $\begin{array}{l}2891.9 \\
2712.0\end{array}$ & $\begin{array}{l}5.8^{\dagger} \\
8.8\end{array}$ & $\begin{array}{l}- \\
-\end{array}$ & $\begin{array}{l}58.6 \\
50.2\end{array}$ & $\begin{array}{l}0.3 \\
0.2\end{array}$ & $\begin{array}{l}57.9 \\
57.6\end{array}$ & $\begin{array}{c}56.6^{\dagger} \\
132.9\end{array}$ & $\begin{array}{l}- \\
-\end{array}$ & $\begin{array}{l}58.3 \\
57.2\end{array}$ & $\begin{array}{l}137.8 \\
170.2\end{array}$ & $\begin{array}{l}573.6 \\
498.3\end{array}$ & $\begin{array}{l}- \\
-\end{array}$ & $\begin{array}{l}948.9 \\
975.5\end{array}$ \\
\hline
\end{tabular}

Financial assets - continued

\begin{tabular}{|c|c|c|c|c|c|c|c|c|c|c|c|c|c|}
\hline & \multicolumn{6}{|c|}{ Loans } & \multicolumn{5}{|c|}{ Shares and other equity } & \multirow{3}{*}{$\begin{array}{l}\text { Other } \\
\text { accounts } \\
\text { receivabl- } \\
\text { e/payable }\end{array}$} & \multirow[b]{3}{*}{$\begin{array}{r}\text { Total } \\
\text { financial } \\
\text { assets }\end{array}$} \\
\hline & \multirow{2}{*}{$\begin{array}{l}\text { Short term } \\
\text { loans } \\
\\
\text { by UK } \\
\text { MFI's }^{3}\end{array}$} & \multicolumn{4}{|c|}{ Long term loans } & \multirow[b]{2}{*}{ Total } & \multicolumn{3}{|c|}{ Excl mutual funds' shares } & \multirow[b]{2}{*}{$\begin{array}{r}\text { UK mutual } \\
\text { funds } \\
\text { shares }\end{array}$} & \multirow[b]{2}{*}{ Total } & & \\
\hline & & $\begin{array}{r}\text { Direct } \\
\text { investment }\end{array}$ & $\begin{array}{l}\text { Secured on } \\
\text { dwellings }\end{array}$ & $\begin{array}{r}\text { Finance } \\
\text { leasing }\end{array}$ & Other & & $\begin{array}{r}\text { Quoted UK } \\
\text { shares }\end{array}$ & $\begin{array}{l}\text { Unquoted } \\
\text { UK shares }\end{array}$ & $\begin{array}{r}\text { By the } \\
\text { rest of } \\
\text { the world }\end{array}$ & & & & \\
\hline & $\mathrm{F} 411$ & $\mathrm{~F} 421$ & $\mathrm{~F} 422$ & $\mathrm{~F} 423$ & $\mathrm{~F} 424$ & $\mathrm{~F} 4$ & F514 & F515 & F519 & $\mathrm{F} 521$ & F5 & $\mathrm{F} 7$ & $\mathrm{FA}$ \\
\hline $\begin{array}{l}2002 \\
2003 \\
2004 \\
2005 \\
2006\end{array}$ & $\begin{aligned} \text { NNUK } \\
1142.5 \\
1283.9 \\
1487.7 \\
1754.5 \\
1963.8\end{aligned}$ & $\begin{array}{r}\text { NNUQ } \\
- \\
- \\
- \\
- \\
-\end{array}$ & $\begin{array}{l}\text { NNUT } \\
591.2 \\
653.4 \\
708.4 \\
749.0 \\
795.5\end{array}$ & $\begin{array}{r}\text { NNUX } \\
2.7 \\
2.7 \\
2.6 \\
2.6 \\
2.6\end{array}$ & $\begin{array}{r}\text { LNPN } \\
3.8 \\
3.7 \\
4.2 \\
4.3 \\
3.4\end{array}$ & $\begin{array}{lr} & \text { NNUI } \\
1 & 740.2 \\
1 & 943.6 \\
2 & 202.9 \\
2 & 510.4 \\
2 & 765.3\end{array}$ & $\begin{array}{r}\text { NNVF } \\
3.3 \\
9.6 \\
8.6 \\
16.6 \\
22.7\end{array}$ & $\begin{array}{r}\text { NNVG } \\
70.7 \\
89.4 \\
108.8 \\
113.8 \\
153.0\end{array}$ & $\begin{array}{r}\text { NNVK } \\
26.4 \\
44.2 \\
87.4 \\
129.1 \\
164.7\end{array}$ & $\begin{array}{r}\text { NNVO } \\
0.9 \\
1.0 \\
1.2 \\
1.5 \\
1.7\end{array}$ & $\begin{array}{l}\text { NNVA } \\
101.3 \\
144.2 \\
205.9 \\
261.1 \\
342.2\end{array}$ & $\begin{array}{r}\text { NNVU } \\
0.8 \\
0.6 \\
0.5 \\
0.5 \\
0.3\end{array}$ & $\begin{array}{r}\text { NNST } \\
3763.9 \\
4316.6 \\
4877.2 \\
5706.4 \\
6529.7\end{array}$ \\
\hline $\begin{array}{l}2007 \\
2008\end{array}$ & $\begin{array}{l}2539.0^{\dagger} \\
3013.9^{\dagger}\end{array}$ & $\begin{array}{l}- \\
-\end{array}$ & $\begin{array}{l}829.7 \\
795.2^{\dagger}\end{array}$ & $\begin{array}{l}2.6 \\
2.6\end{array}$ & $\begin{array}{l}3.3 \\
4.5\end{array}$ & $\begin{array}{l}3 \text { 374.6 } \\
3816.2^{\dagger}\end{array}$ & $\begin{array}{l}26.2 \\
11.8\end{array}$ & $\begin{array}{l}123.2^{\dagger} \\
124.7^{\dagger}\end{array}$ & $\begin{array}{l}191.8 \\
113.2\end{array}$ & $\begin{array}{l}1.7 \\
1.0\end{array}$ & $\begin{array}{l}342.8 \\
250.7^{\dagger}\end{array}$ & $\begin{array}{l}0.2 \\
0.2^{\dagger}\end{array}$ & $\begin{array}{ll}7 & 216.6 \\
8 & 046.6^{\dagger}\end{array}$ \\
\hline $\begin{array}{r}2006 \text { Q1 } \\
\text { Q2 } \\
\text { Q3 } \\
\text { Q4 }\end{array}$ & $\begin{array}{l}1888.6 \\
1880.9 \\
1944.4 \\
1963.8\end{array}$ & $\begin{array}{l}- \\
- \\
- \\
-\end{array}$ & $\begin{array}{l}746.9 \\
764.2 \\
783.6 \\
795.5\end{array}$ & $\begin{array}{l}2.6 \\
2.6 \\
2.6 \\
2.6\end{array}$ & $\begin{array}{l}4.1 \\
3.8 \\
3.5 \\
3.4\end{array}$ & $\begin{array}{l}2642.2 \\
2651.6 \\
2734.1 \\
2765.3\end{array}$ & $\begin{array}{l}16.3 \\
17.6 \\
17.3 \\
22.7\end{array}$ & $\begin{array}{l}143.7 \\
152.3 \\
149.1 \\
153.0\end{array}$ & $\begin{array}{l}143.2 \\
135.4 \\
150.3 \\
164.7\end{array}$ & $\begin{array}{l}1.7 \\
1.6 \\
1.6 \\
1.7\end{array}$ & $\begin{array}{l}304.8 \\
306.9 \\
318.4 \\
342.2\end{array}$ & $\begin{array}{l}0.3 \\
0.3 \\
0.3 \\
0.3\end{array}$ & 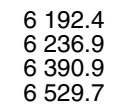 \\
\hline $\begin{array}{r}2007 \text { Q1 } \\
\text { Q2 } \\
\text { Q3 } \\
\text { Q4 }\end{array}$ & 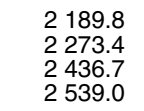 & $\begin{array}{l}- \\
- \\
- \\
-\end{array}$ & $\begin{array}{l}801.8 \\
806.7 \\
819.2 \\
829.7\end{array}$ & $\begin{array}{l}2.6 \\
2.6 \\
2.6 \\
2.6\end{array}$ & $\begin{array}{l}3.4 \\
3.3 \\
3.2 \\
3.3\end{array}$ & $\begin{array}{l}2997.5 \\
35086.1 \\
3261.7 \\
3374.6\end{array}$ & $\begin{array}{l}27.6 \\
26.7 \\
27.8 \\
26.2\end{array}$ & $\begin{array}{l}116.7 \\
125.0 \\
118.8 \\
123.2\end{array}$ & $\begin{array}{l}174.8 \\
182.0 \\
179.6 \\
191.8\end{array}$ & $\begin{array}{l}1.8 \\
1.8 \\
1.8 \\
1.7\end{array}$ & $\begin{array}{l}321.0 \\
335.5 \\
328.1 \\
342.8\end{array}$ & $\begin{array}{l}0.2 \\
0.2 \\
0.2 \\
0.2\end{array}$ & $\begin{array}{ll}7 & 057.8 \\
7 & 142.6 \\
7 & 027.5 \\
7 & 216.6\end{array}$ \\
\hline $\begin{array}{r}2008 \text { Q1 } \\
\text { Q2 } \\
\text { Q3 } \\
\text { Q4 }\end{array}$ & $\begin{array}{l}2763.5^{\dagger} \\
2630.8 \\
2810.0 \\
3013.9\end{array}$ & $\begin{array}{l}- \\
- \\
- \\
-\end{array}$ & $\begin{array}{l}849.5 \\
853.1 \\
831.9^{\dagger} \\
795.2^{\dagger}\end{array}$ & $\begin{array}{l}2.6 \\
2.6 \\
2.6 \\
2.6\end{array}$ & $\begin{array}{l}3.4 \\
3.4 \\
3.7 \\
4.5\end{array}$ & $\begin{array}{l}3619.1^{\dagger} \\
35490.0 \\
3648.3 \\
3816.2\end{array}$ & $\begin{array}{l}19.4 \\
24.9 \\
17.4 \\
11.8\end{array}$ & $\begin{array}{l}121.3 \\
122.2 \\
120.2 \\
124.7^{\dagger}\end{array}$ & $\begin{array}{l}148.4 \\
142.8 \\
137.3 \\
113.2\end{array}$ & $\begin{array}{l}1.6 \\
1.5 \\
1.2 \\
1.0\end{array}$ & $\begin{array}{l}290.7 \\
291.3 \\
276.1 \\
250.7^{\dagger}\end{array}$ & $\begin{array}{l}0.2 \\
0.2^{\dagger} \\
0.2 \\
0.2\end{array}$ & $\begin{array}{ll}7 & 668.4^{\dagger} \\
7 & 182.9 \\
7 & 634.7 \\
8 & 046.6\end{array}$ \\
\hline $\begin{array}{r}2009 \text { Q1 } \\
\text { Q2 }\end{array}$ & $\begin{array}{l}2915.8 \\
2598.3\end{array}$ & $\begin{array}{l}- \\
-\end{array}$ & $\begin{array}{l}800.6 \\
900.3\end{array}$ & $\begin{array}{l}2.6 \\
2.6\end{array}$ & $\begin{array}{l}4.3 \\
3.8\end{array}$ & $\begin{array}{l}3723.3 \\
3505.0\end{array}$ & $\begin{array}{l}11.9 \\
16.2\end{array}$ & $\begin{array}{l}124.7 \\
143.3\end{array}$ & $\begin{array}{l}111.2 \\
113.4\end{array}$ & $\begin{array}{l}1.0 \\
1.1\end{array}$ & $\begin{array}{l}248.7 \\
274.0\end{array}$ & $\begin{array}{l}0.2 \\
0.2\end{array}$ & $\begin{array}{l}7813.0 \\
7466.7\end{array}$ \\
\hline $\begin{array}{l}1 \text { Monet } \\
2 \text { Money } \\
3 \text { All loa } \\
\text { term Ic }\end{array}$ & $\begin{array}{l}\text { nancial inst } \\
\text { ket instrum } \\
\text { ecured on }\end{array}$ & $\begin{array}{l}\text { litutions } \\
\text { ents } \\
\text { dwellings }\end{array}$ & 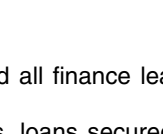 & - & 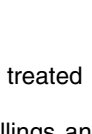 & long & & & & & Source & $\begin{array}{r}\text { es: National } \\
\text { Bank of }\end{array}$ & $\begin{array}{l}\text { Statistics; } \\
\text { f England }\end{array}$ \\
\hline
\end{tabular}

4 Other than direct investment loans, loans secured on dwellings and loans for finance leasing 


\begin{tabular}{|c|c|c|c|c|c|c|c|c|c|c|c|c|c|}
\hline & \multicolumn{13}{|c|}{ Financial liabilities (MFI) } \\
\hline & \multicolumn{3}{|c|}{ Currency and deposits } & \multicolumn{5}{|c|}{ Securities other than shares } & \multicolumn{5}{|c|}{ Loans } \\
\hline & & & & $\begin{array}{l}\text { Short term } \\
\text { MMl's }^{2} \\
\text { issued }\end{array}$ & Bonds is & ssued & & & $\begin{array}{l}\text { Short term } \\
\text { loans }\end{array}$ & Lon & ng term loa & ans & \\
\hline & Currency & $\begin{array}{c}\text { Deposits } \\
\text { with UK } \\
\text { MFIs }^{1}\end{array}$ & Total & by UK MFIs & $\begin{array}{r}\text { medium } \\
\text { term by UK } \\
\text { MFIs }\end{array}$ & $\begin{array}{r}\text { other by } \\
\text { UK } \\
\text { residents }\end{array}$ & $\begin{array}{r}\text { Financial } \\
\text { derivativ- } \\
\text { es }\end{array}$ & Total & by UK MFIs ${ }^{3}$ & $\begin{array}{r}\text { Direct } \\
\text { investment }\end{array}$ & $\begin{array}{r}\text { Finance } \\
\text { leasing }\end{array}$ & $\begin{array}{r}\text { by UK } \\
\text { residents }{ }^{4}\end{array}$ & Total \\
\hline & F21 & F221 & $\mathrm{F} 2$ & F3315 & F3325 & F3326 & F34 & F3 & F411 & F421 & F423 & F424 & F4 \\
\hline $\begin{array}{l}2002 \\
2003 \\
2004 \\
2005 \\
2006\end{array}$ & $\begin{array}{r}\text { NNWC } \\
37.9 \\
40.8 \\
46.3 \\
47.2 \\
49.0\end{array}$ & $\begin{array}{l}\text { NNWE } \\
3034.7 \\
3518.7 \\
3981.1 \\
4673.7 \\
5328.5\end{array}$ & 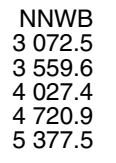 & $\begin{array}{r}\text { NNWR } \\
302.6 \\
282.1 \\
283.2 \\
291.7 \\
327.7\end{array}$ & $\begin{array}{r}\text { NNXD } \\
81.0 \\
107.0 \\
134.7 \\
175.4 \\
205.8\end{array}$ & $\begin{array}{r}\text { NNXG } \\
92.2 \\
113.2 \\
119.4 \\
138.8 \\
143.4\end{array}$ & $\begin{array}{r}\text { NNXI } \\
- \\
- \\
0.1 \\
0.2 \\
0.7\end{array}$ & $\begin{array}{r}\text { NNWK } \\
475.8 \\
502.4 \\
537.5 \\
606.3 \\
677.6\end{array}$ & $\begin{array}{r}\text { NNXO } \\
- \\
- \\
- \\
- \\
-\end{array}$ & $\begin{array}{r}\text { NNXU } \\
0.9 \\
1.2 \\
1.3 \\
1.3 \\
1.3\end{array}$ & $\begin{array}{r}\text { NNYB } \\
1.5 \\
1.7 \\
1.8 \\
1.9 \\
2.0\end{array}$ & $\begin{array}{r}\text { NNYC } \\
- \\
- \\
- \\
- \\
-\end{array}$ & $\begin{array}{r}\text { NNXM } \\
2.4 \\
2.9 \\
3.1 \\
3.2 \\
3.3\end{array}$ \\
\hline 2005 Q4 & 47.2 & 4673.7 & 4720.9 & 291.7 & 175.4 & 138.8 & 0.2 & 606.3 & - & 1.3 & 1.9 & - & 3.2 \\
\hline $\begin{array}{r}2006 \text { Q1 } \\
\text { Q2 } \\
\text { Q3 } \\
\text { Q4 }\end{array}$ & $\begin{array}{l}45.3 \\
45.8 \\
46.3 \\
49.0\end{array}$ & $\begin{array}{ll}5 & 086.6 \\
5 & 143.1 \\
5 & 236.5 \\
5 & 328.5\end{array}$ & $\begin{array}{ll}5 & 131.9 \\
5 & 189.0 \\
5 & 282.8 \\
5 & 377.5\end{array}$ & $\begin{array}{l}315.1 \\
309.1 \\
334.9 \\
327.7\end{array}$ & $\begin{array}{l}195.5 \\
187.6 \\
196.3 \\
205.8\end{array}$ & $\begin{array}{l}131.4 \\
143.2 \\
140.3 \\
143.4\end{array}$ & $\begin{array}{l}0.3 \\
0.4 \\
0.6 \\
0.7\end{array}$ & $\begin{array}{l}642.3 \\
640.3 \\
672.1 \\
677.6\end{array}$ & $\begin{array}{l}- \\
- \\
- \\
-\end{array}$ & $\begin{array}{l}1.3 \\
1.3 \\
1.3 \\
1.3\end{array}$ & $\begin{array}{l}1.9 \\
1.9 \\
1.9 \\
2.0\end{array}$ & $\begin{array}{l}- \\
- \\
- \\
-\end{array}$ & $\begin{array}{l}3.2 \\
3.2 \\
3.3 \\
3.3\end{array}$ \\
\hline $\begin{array}{r}2007 \text { Q1 } \\
\text { Q2 } \\
\text { Q3 } \\
\text { Q4 }\end{array}$ & $\begin{array}{l}47.1 \\
48.2 \\
49.0 \\
50.0\end{array}$ & $\begin{array}{l}5824.8 \\
5858.9 \\
5698.8 \\
5895.4\end{array}$ & $\begin{array}{l}5871.9 \\
5907.1 \\
5747.8 \\
5945.4\end{array}$ & $\begin{array}{l}340.0 \\
340.7 \\
363.2 \\
348.5\end{array}$ & $\begin{array}{l}223.7 \\
255.1 \\
277.9 \\
285.3\end{array}$ & $\begin{array}{l}139.0 \\
119.3 \\
108.0 \\
112.9\end{array}$ & $\begin{array}{r}0.6 \\
0.8 \\
0.2 \\
-0.5\end{array}$ & $\begin{array}{l}703.4 \\
715.9 \\
749.2 \\
746.3\end{array}$ & $\begin{array}{l}- \\
- \\
- \\
-\end{array}$ & $\begin{array}{l}1.3 \\
1.3 \\
1.3 \\
1.3\end{array}$ & $\begin{array}{l}2.0 \\
2.0 \\
2.1 \\
2.1\end{array}$ & $\begin{array}{l}- \\
- \\
-\end{array}$ & $\begin{array}{l}3.3 \\
3.3 \\
3.3 \\
3.3\end{array}$ \\
\hline $\begin{array}{r}2008 \text { Q1 } \\
\text { Q2 } \\
\text { Q3 } \\
\text { Q4 }\end{array}$ & $\begin{array}{l}47.5 \\
47.9 \\
48.1 \\
52.3\end{array}$ & $\begin{array}{l}6351.5^{\dagger} \\
5867.3 \\
6348.2 \\
6695.9\end{array}$ & $\begin{array}{l}6399.0^{\dagger} \\
5915.1 \\
6396.3 \\
6748.2\end{array}$ & $\begin{array}{l}394.3^{\dagger} \\
377.4 \\
367.6 \\
359.8\end{array}$ & $\begin{array}{l}286.1 \\
293.5 \\
292.8 \\
357.6\end{array}$ & $\begin{array}{l}134.5^{\dagger} \\
197.5^{\dagger} \\
220.4 \\
223.8\end{array}$ & $\begin{array}{l}-1.5 \\
-1.0 \\
-1.2 \\
-3.5\end{array}$ & $\begin{array}{l}813.4^{\dagger} \\
867.4 \\
879.6 \\
937.6\end{array}$ & $\begin{array}{l}- \\
- \\
- \\
-\end{array}$ & $\begin{array}{l}1.3 \\
1.3 \\
1.2 \\
1.2\end{array}$ & $\begin{array}{l}2.1 \\
2.1 \\
2.2 \\
2.2\end{array}$ & $\begin{array}{l}- \\
- \\
- \\
-\end{array}$ & $\begin{array}{l}3.4 \\
3.4 \\
3.3 \\
3.3\end{array}$ \\
\hline $\begin{array}{r}2009 \text { Q1 } \\
\text { Q2 }\end{array}$ & $\begin{array}{l}51.0 \\
52.2\end{array}$ & $\begin{array}{l}6487.4 \\
6091.0\end{array}$ & $\begin{array}{l}6538.4 \\
6143.2\end{array}$ & $\begin{array}{l}404.3 \\
382.7\end{array}$ & $\begin{array}{l}363.1^{\dagger} \\
369.4\end{array}$ & $\begin{array}{l}274.6 \\
283.6\end{array}$ & $\begin{array}{l}-2.8 \\
-1.5\end{array}$ & $\begin{array}{l}1039.1 \\
1034.3\end{array}$ & $\begin{array}{l}- \\
-\end{array}$ & $\begin{array}{l}1.1 \\
1.1\end{array}$ & $\begin{array}{l}2.2 \\
2.2\end{array}$ & $\begin{array}{l}- \\
-\end{array}$ & $\begin{array}{l}3.3 \\
3.3\end{array}$ \\
\hline
\end{tabular}

Financial liabilities - continued

Shares and other equity

Excl mutual funds' shares

\begin{tabular}{|c|c|c|c|c|c|c|c|}
\hline & Quoted UK companies & $\begin{array}{r}\text { Unquoted UK } \\
\text { companies }\end{array}$ & Total & $\begin{array}{l}\text { Other accounts } \\
\text { receivable/payable }\end{array}$ & $\begin{array}{r}\text { Total financial } \\
\text { liabilities }\end{array}$ & $\begin{array}{r}\text { Total financial } \\
\text { assets }\end{array}$ & $\begin{array}{r}\text { Net assets } \\
(+) / \text { liabilities(-) }\end{array}$ \\
\hline & F514 & F515 & F5 & $\mathrm{F} 7$ & $\mathrm{FL}$ & $\mathrm{FA}$ & BF90 \\
\hline $\begin{array}{l}2002 \\
2003 \\
2004 \\
2005 \\
2006\end{array}$ & $\begin{array}{r}\text { NNYJ } \\
19.4 \\
20.8 \\
14.0 \\
11.2 \\
13.5\end{array}$ & $\begin{array}{l}\text { NNYK } \\
109.1 \\
108.0 \\
119.8 \\
124.5 \\
126.8\end{array}$ & $\begin{array}{l}\text { NNYE } \\
128.5 \\
128.8 \\
133.8 \\
135.7 \\
140.2\end{array}$ & $\begin{array}{r}\text { NNYY } \\
3.9 \\
4.0 \\
4.9 \\
5.8 \\
6.2\end{array}$ & $\begin{array}{r}\text { NNVX } \\
3683.1 \\
4197.7 \\
4706.7 \\
5 \quad 471.9 \\
6204.8\end{array}$ & $\begin{array}{r}\text { NNST } \\
3763.9 \\
4316.6 \\
4877.2 \\
5706.4 \\
6529.7\end{array}$ & $\begin{array}{r}\text { NYOL } \\
80.8 \\
118.9 \\
170.5 \\
234.5 \\
324.9\end{array}$ \\
\hline $\begin{array}{l}2007 \\
2008\end{array}$ & $\begin{array}{l}5.0 \\
0.1\end{array}$ & $\begin{array}{l}133.3^{\dagger} \\
144.2^{\dagger}\end{array}$ & $\begin{array}{l}138.3^{\dagger} \\
144.3^{\dagger}\end{array}$ & $26.5^{\dagger}$ & $\begin{array}{l}6840.9^{\dagger} \\
7860.0^{\dagger}\end{array}$ & $\begin{array}{l}7216.6 \\
8046.6^{\dagger}\end{array}$ & $\begin{array}{l}375.6 \\
186.6\end{array}$ \\
\hline 2005 Q4 & 11.2 & 124.5 & 135.7 & 5.8 & 5471.9 & 5706.4 & 234.5 \\
\hline $\begin{array}{r}2006 \text { Q1 } \\
\text { Q2 } \\
\text { Q3 } \\
\text { Q4 }\end{array}$ & $\begin{array}{l}13.9 \\
12.8 \\
13.2 \\
13.5\end{array}$ & $\begin{array}{l}123.9 \\
125.8 \\
126.4 \\
126.8\end{array}$ & $\begin{array}{l}137.8 \\
138.6 \\
139.6 \\
140.2\end{array}$ & $\begin{array}{l}4.9 \\
4.7 \\
5.4 \\
6.2\end{array}$ & 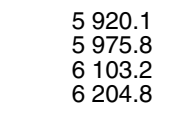 & $\begin{array}{l}6192.4 \\
6236.9 \\
6390.9 \\
6529.7\end{array}$ & $\begin{array}{l}272.3 \\
261.1 \\
287.7 \\
324.9\end{array}$ \\
\hline $\begin{array}{r}2008 \text { Q1 } \\
\text { Q2 } \\
\text { Q3 } \\
\text { Q4 }\end{array}$ & $\begin{array}{l}3.5 \\
1.8 \\
1.3 \\
0.1\end{array}$ & $\begin{array}{l}134.0 \\
135.9 \\
140.3^{\dagger} \\
144 . .^{\dagger}\end{array}$ & $\begin{array}{l}137.5 \\
137.7 \\
141.5 \\
144.3^{\dagger}\end{array}$ & $\begin{array}{r}6.5^{\dagger} \\
6.3 \\
21.0 \\
26.5\end{array}$ & $\begin{array}{l}7359.9^{\dagger} \\
6929.9 \\
7441.8 \\
7860.0\end{array}$ & $\begin{array}{l}7668.4^{\dagger} \\
7182.9 \\
7634.7 \\
8046.6\end{array}$ & $\begin{array}{l}308.5 \\
253.0 \\
192.9 \\
186.6\end{array}$ \\
\hline $\begin{array}{r}2009 \text { Q1 } \\
\text { Q2 }\end{array}$ & $\begin{array}{l}0.1 \\
0.1\end{array}$ & $\begin{array}{l}144.6 \\
144.3\end{array}$ & $\begin{array}{l}144.7 \\
144.4\end{array}$ & $\begin{array}{l}24.4 \\
23.2\end{array}$ & $\begin{array}{l}7750.0 \\
7348.3\end{array}$ & $\begin{array}{l}7813.0 \\
7466.7\end{array}$ & $\begin{array}{r}63.0 \\
118.4\end{array}$ \\
\hline
\end{tabular}

1 Monetary financial institutions

2 Money market instruments

Sources: National Statistics

3 All loans secured on dwellings and all finance leasing are treated as long

4 Other than direct investment loans, loans secured on dwellings and loans

for finance leasing 


\begin{tabular}{|c|c|c|c|c|c|c|c|c|c|c|c|c|c|c|c|c|c|}
\hline & \multicolumn{17}{|c|}{ Financial assets (banks) } \\
\hline & \multicolumn{6}{|c|}{ Currency and deposits } & \multicolumn{11}{|c|}{ Securities other than shares } \\
\hline & \multirow[b]{2}{*}{$\begin{array}{r}\text { Curren- } \\
\text { cy }\end{array}$} & \multirow[b]{2}{*}{$\begin{array}{r}\text { Sterli- } \\
\text { ng Bank } \\
\text { deposi- } \\
\text { ts with } \\
\text { UK } \\
\text { MFI's }^{1}\end{array}$} & \multirow[b]{2}{*}{$\begin{array}{l}\text { Foreign } \\
\text { curren- } \\
\text { cy Bank }\end{array}$} & \multirow[b]{2}{*}{$\begin{array}{r}\text { Sterli- } \\
\text { ng } \\
\text { B.socs } \\
\text { deposi- } \\
\text { ts }\end{array}$} & \multirow[b]{2}{*}{$\begin{array}{l}\text { Deposi- } \\
\text { ts with } \\
\text { rest of } \\
\text { the } \\
\text { world } \\
\text { MFl's }\end{array}$} & \multirow[b]{2}{*}{$\begin{array}{r}\text { Deposi- } \\
\text { ts } \\
\text { other } \\
\text { than } \\
\text { with } \\
\text { MFI's }\end{array}$} & \multicolumn{5}{|c|}{ Short term MMl's ${ }^{2}$ issued } & \multicolumn{5}{|c|}{ Bonds issued } & \multirow[b]{2}{*}{ Total } \\
\hline & & & & & & & $\begin{array}{l}\text { by UK } \\
\text { general a } \\
\text { govern- } \\
\text { ment }\end{array}$ & $\begin{array}{r}\text { UK } \\
\text { local } \\
\text { author- } \\
\text { ity } \\
\text { bills }\end{array}$ & $\begin{array}{l}\text { by UK r } \\
\text { MFI's }\end{array}$ & $\begin{array}{l}\text { by } \\
\text { other } \\
\text { UK r } \\
\text { reside- } \\
\text { nts }\end{array}$ & $\begin{array}{l}\text { by the } \\
\text { rest of } \\
\text { the } \\
\text { world }\end{array}$ & $\begin{array}{c}\text { by UK } \\
\text { central } \\
\text { govern- a } \\
\text { ment }\end{array}$ & $\begin{array}{r}\text { by UK } \\
\text { local } \\
\text { author- } \\
\text { ities }\end{array}$ & $\begin{array}{c}\text { Medium I } \\
\text { term by } \\
\text { banks }\end{array}$ & $\begin{array}{l}\text { Medium } \\
\text { term by } r \\
\text { B.socs }\end{array}$ & $\begin{array}{l}\text { Other by the } \\
\text { by UK rest of } \\
\text { reside- the } \\
\text { nts world }\end{array}$ & \\
\hline & $\mathrm{F} 21$ & $\mathrm{~F} 2211$ & F2212 & F2213 & F229 & F29 & F3311 & F3312 & F3315 & F3316 F & F3319 & F3321 & F3322 & F33251 & F33252 & F3326 F3329 & F3 \\
\hline $\begin{array}{l}2002 \\
2003 \\
2004 \\
2005 \\
2006\end{array}$ & $\begin{array}{r}\text { NHSQ } \\
6.7 \\
7.6 \\
10.7 \\
9.6 \\
9.4\end{array}$ & $\begin{array}{l}\text { NHST } \\
297.1 \\
361.4 \\
429.3 \\
574.9 \\
830.6\end{array}$ & $\begin{array}{r}\text { NHSU } \\
202.0 \\
364.0 \\
392.8 \\
414.6 \\
440.7\end{array}$ & $\begin{array}{r}\text { NHSV } \\
1.5 \\
3.4 \\
2.4 \\
2.3 \\
2.9\end{array}$ & $\begin{array}{r}\text { NHSW } \\
842.7 \\
921.2 \\
1006.6 \\
1199.4 \\
1285.6\end{array}$ & $\begin{array}{r}\text { NHSX NHSP } \\
-1350.1 \\
-1657.6 \\
-1841.8 \\
-2200.9 \\
-2569.3\end{array}$ & $\begin{array}{r}\text { NHTA } \\
19.3 \\
18.3 \\
16.1 \\
15.7 \\
12.0\end{array}$ & $\begin{array}{r}\text { NHTE } \\
- \\
- \\
- \\
- \\
-\end{array}$ & $\begin{array}{r}\text { NHTF } \\
91.2 \\
75.6 \\
76.9 \\
81.3 \\
81.9\end{array}$ & $\begin{array}{r}\text { NHTK } \\
0.7 \\
2.8 \\
1.6 \\
2.5 \\
4.6\end{array}$ & $\begin{array}{r}\text { NHTL } \\
32.3 \\
40.6 \\
35.2 \\
37.9 \\
40.4\end{array}$ & $\begin{array}{r}\text { NHTN } \\
-1.2 \\
-6.7 \\
-2.4 \\
-4.1 \\
-8.7\end{array}$ & $\begin{array}{r}\text { NHTQ } \\
- \\
- \\
- \\
- \\
-\end{array}$ & $\begin{array}{r}\text { NHTS } \\
11.1 \\
12.2 \\
13.2 \\
13.3 \\
12.3\end{array}$ & $\begin{array}{r}\text { NHTT } \\
2.7 \\
2.9 \\
3.8 \\
4.3 \\
4.0\end{array}$ & $\begin{array}{rr}\text { NHTU } & \text { NHTV } \\
46.8 & 330.0 \\
60.6 & 322.5 \\
84.4 & 354.1 \\
127.2 & 407.8 \\
180.9 & 471.7\end{array}$ & $\begin{array}{l}\text { NHSY } \\
532.9 \\
528.7 \\
582.8 \\
686.1 \\
798.9\end{array}$ \\
\hline $\begin{array}{l}2007 \\
2008\end{array}$ & $\begin{array}{l}8.0 \\
7.6\end{array}$ & $\begin{array}{l}398.3 \\
422.1\end{array}$ & $\begin{array}{l}430.5 \\
479.5\end{array}$ & $\begin{array}{l}1.9 \\
2.0\end{array}$ & $\begin{array}{l}1712.3^{\dagger} \\
2091.3^{\dagger}\end{array}$ & $\begin{array}{l}-2551.0^{\dagger} \\
-3002.5^{\dagger}\end{array}$ & $\begin{array}{l}4.8 \\
2.8\end{array}$ & $\begin{array}{l}- \\
-\end{array}$ & $\begin{array}{l}70.9 \\
60.6\end{array}$ & $\begin{array}{l}0.9 \\
0.5\end{array}$ & $\begin{array}{l}52.4 \\
54.2\end{array}$ & $\begin{array}{r}-10.5 \\
17.9\end{array}$ & $\begin{array}{l}- \\
-\end{array}$ & $\begin{array}{l}14.2 \\
31.5\end{array}$ & $\begin{array}{l}3.0 \\
3.0\end{array}$ & $\begin{array}{l}200.2+545.2 \\
173.0^{\dagger} 556.2\end{array}$ & $\begin{array}{l}881.0^{\dagger} \\
899.7^{\dagger}\end{array}$ \\
\hline 2005 Q4 & 9.6 & 574.9 & 414.6 & 2.3 & 1199.4 & -2200.9 & 15.7 & - & 81.3 & 2.5 & 37.9 & -4.1 & - & 13.3 & 4.3 & 127.2407 .8 & 686.1 \\
\hline $\begin{array}{r}2006 \text { Q1 } \\
\text { Q2 } \\
\text { Q3 } \\
\text { Q4 }\end{array}$ & $\begin{array}{l}8.3 \\
8.3 \\
8.6 \\
9.4\end{array}$ & $\begin{array}{l}628.6 \\
699.0 \\
726.3 \\
830.6\end{array}$ & $\begin{array}{l}491.3 \\
466.0 \\
460.1 \\
440.7\end{array}$ & $\begin{array}{l}3.4 \\
3.4 \\
3.3 \\
2.9\end{array}$ & $\begin{array}{ll}1 & 329.4 \\
1 & 309.1 \\
1 & 337.7 \\
1 & 285.6\end{array}$ & $\begin{array}{l}-2461.0 \\
-2485.9 \\
-2536.0 \\
-2569.3\end{array}$ & $\begin{array}{l}11.9 \\
11.5 \\
11.8 \\
12.0\end{array}$ & $\begin{array}{l}- \\
- \\
- \\
-\end{array}$ & $\begin{array}{l}90.5 \\
85.2 \\
85.5 \\
81.9\end{array}$ & $\begin{array}{l}3.1 \\
2.0 \\
3.2 \\
4.6\end{array}$ & $\begin{array}{l}43.0 \\
44.2 \\
41.6 \\
40.4\end{array}$ & $\begin{array}{l}-5.8 \\
-1.6 \\
-3.8 \\
-8.7\end{array}$ & $\begin{array}{l}- \\
- \\
- \\
-\end{array}$ & $\begin{array}{l}14.1 \\
11.7 \\
11.8 \\
12.3\end{array}$ & $\begin{array}{l}4.2 \\
3.9 \\
3.6 \\
4.0\end{array}$ & $\begin{array}{ll}153.3 & 423.8 \\
155.3 & 431.6 \\
165.2 & 433.9 \\
180.9 & 471.7\end{array}$ & $\begin{array}{l}738.0 \\
743.8 \\
752.8 \\
798.9\end{array}$ \\
\hline $\begin{array}{r}2007 \text { Q1 } \\
\text { Q2 } \\
\text { Q3 } \\
\text { Q4 }\end{array}$ & $\begin{array}{l}8.8 \\
8.9 \\
8.8 \\
8.0\end{array}$ & $\begin{array}{l}850.1 \\
826.4 \\
467.9 \\
398.3\end{array}$ & $\begin{array}{l}520.5 \\
481.0 \\
454.4 \\
430.5\end{array}$ & $\begin{array}{l}3.5 \\
4.4 \\
3.5 \\
1.9\end{array}$ & $\begin{array}{ll}1 & 460.8 \\
1 & 483.6 \\
1 & 578.2 \\
1 & 712.3\end{array}$ & $\begin{array}{l}-2843.6 \\
-2804.3 \\
-2512.8 \\
-2551.0\end{array}$ & $\begin{array}{l}8.1 \\
8.0 \\
4.6 \\
4.8\end{array}$ & $\begin{array}{l}- \\
- \\
- \\
-\end{array}$ & $\begin{array}{l}87.9 \\
76.8 \\
83.5 \\
70.9\end{array}$ & $\begin{array}{l}4.8 \\
2.5 \\
1.1 \\
0.9\end{array}$ & $\begin{array}{l}50.1 \\
50.1 \\
49.1 \\
52.4\end{array}$ & $\begin{array}{l}-17.5 \\
-11.2 \\
-10.0 \\
-10.5\end{array}$ & $\begin{array}{l}- \\
- \\
- \\
-\end{array}$ & $\begin{array}{l}13.5 \\
14.4 \\
12.4 \\
14.2\end{array}$ & $\begin{array}{l}3.6 \\
3.8 \\
3.1 \\
3.0\end{array}$ & $\begin{array}{ll}191.1 & 502.0 \\
194.4 & 522.8 \\
197.3 & 527.9 \\
200.2 & 545.2\end{array}$ & $\begin{array}{l}843.7 \\
861.5 \\
869.1 \\
881.0\end{array}$ \\
\hline $\begin{array}{r}2008 \text { Q1 } \\
\text { Q2 } \\
\text { Q3 } \\
\text { Q4 }\end{array}$ & $\begin{array}{l}6.5 \\
6.0 \\
6.3 \\
7.6\end{array}$ & $\begin{array}{l}417.5 \\
384.4 \\
486.3 \\
422.1\end{array}$ & $\begin{array}{l}496.1 \\
400.1 \\
432.6 \\
479.5\end{array}$ & $\begin{array}{l}2.7 \\
4.0 \\
2.8 \\
2.0\end{array}$ & $\begin{array}{ll}1 & 911.7 \\
1 & 720.7 \\
1 & 864.0^{\dagger} \\
2 & 091.3\end{array}$ & $\begin{array}{l}-2834.5 \\
-2515.3 \\
-2792.0^{\dagger} \\
-3002.5\end{array}$ & $\begin{array}{l}4.2 \\
4.0 \\
1.6 \\
2.8\end{array}$ & $\begin{array}{l}- \\
- \\
- \\
-\end{array}$ & $\begin{array}{l}69.5 \\
56.6 \\
60.1 \\
60.6\end{array}$ & $\begin{array}{l}0.9 \\
0.9 \\
0.8 \\
0.5\end{array}$ & $\begin{array}{l}49.8 \\
53.6 \\
57.5 \\
54.2\end{array}$ & $\begin{array}{l}-7.7 \\
-6.9 \\
-1.8 \\
17.9\end{array}$ & $\begin{array}{l}- \\
- \\
- \\
-\end{array}$ & $\begin{array}{l}14.3 \\
15.3 \\
16.5 \\
31.5\end{array}$ & $\begin{array}{l}2.9 \\
2.7 \\
2.7 \\
3.0\end{array}$ & $\begin{array}{ll}173.6 & 543.8 \\
178.5 & 506.4 \\
181.6 & 519.1 \\
173.0 & 556.2\end{array}$ & $\begin{array}{l}851.3^{\dagger} \\
811.1^{\dagger} \\
838.1^{-1} \\
899.7\end{array}$ \\
\hline $\begin{array}{r}2009 \text { Q1 } \\
\text { Q2 }\end{array}$ & $\begin{array}{l}6.1 \\
6.7\end{array}$ & $\begin{array}{l}413.5 \\
495.6\end{array}$ & $\begin{array}{l}411.0 \\
375.4\end{array}$ & $\begin{array}{l}2.1 \\
1.5\end{array}$ & $\begin{array}{l}2039.2 \\
1813.5\end{array}$ & $\begin{array}{l}-2872.0 \\
-2692.6\end{array}$ & $\begin{array}{l}4.8^{\dagger} \\
6.6\end{array}$ & $\begin{array}{l}- \\
-\end{array}$ & $\begin{array}{l}47.4 \\
43.1\end{array}$ & $\begin{array}{l}0.3 \\
0.2\end{array}$ & $\begin{array}{l}54.8 \\
54.6\end{array}$ & $\begin{array}{c}48.0^{\dagger} \\
122.9\end{array}$ & $\begin{array}{l}- \\
-\end{array}$ & $\begin{array}{l}46.3 \\
45.6\end{array}$ & $\begin{array}{l}2.8 \\
2.6\end{array}$ & $\begin{array}{ll}127.2 & 560.7^{\dagger} \\
159.7 & 485.4\end{array}$ & $\begin{array}{l}+ \\
9 \\
920.9\end{array}$ \\
\hline
\end{tabular}

Financial assets - continued (banks)

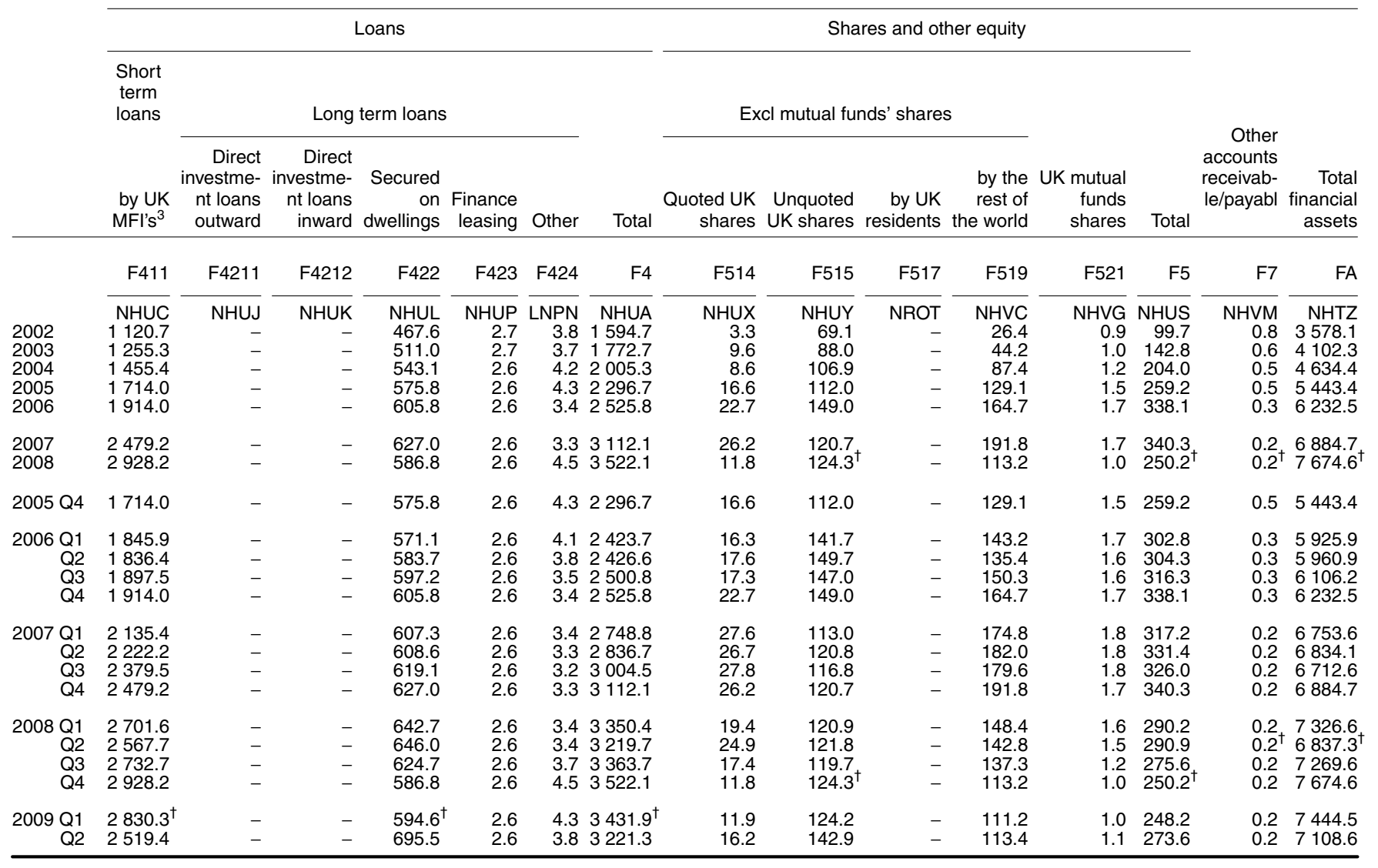

1 Monetary financial institutions

2 Money market instruments

Sources: National Statistics;

3 All loans secured on dwellings and all finance leasing are treated as long 


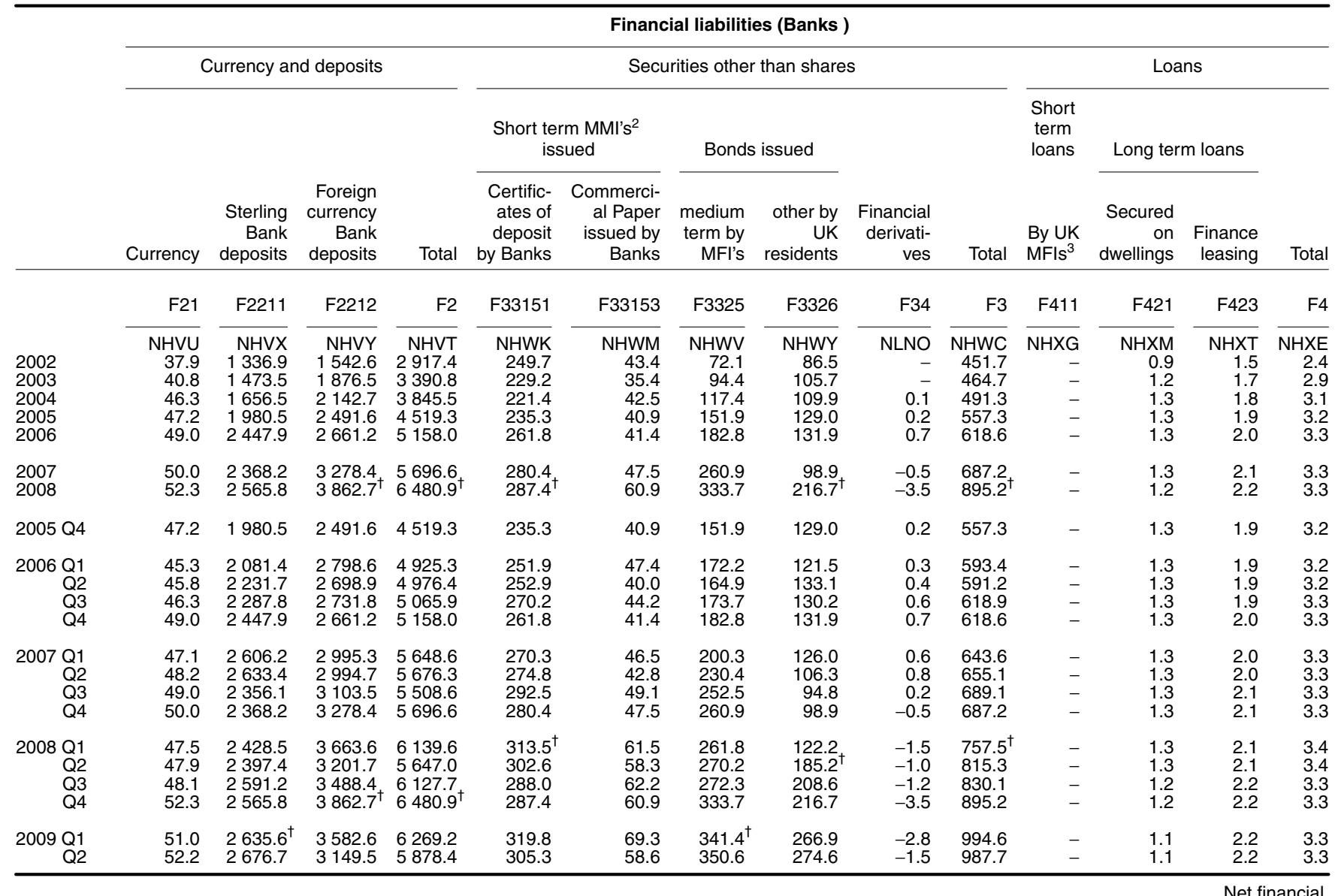

Financial liabilities - continued (banks)

Shares and other equity

Excl mutual funds' shares

\begin{tabular}{|c|c|c|c|c|c|c|c|c|}
\hline & & \multirow{2}{*}{\multicolumn{2}{|c|}{$\begin{array}{r}\text { Other accounts } \\
\text { receivable/paya- } \\
\text { ble }\end{array}$}} & \multirow[b]{2}{*}{$\begin{array}{r}\text { Total financial } \\
\text { liabilities }\end{array}$} & \multirow[b]{2}{*}{$\begin{array}{r}\text { Total financial } \\
\text { assets }\end{array}$} & \multirow{2}{*}{$\begin{array}{r}\text { Net assets } \\
(+) / \text { liabilities- } \\
(-)\end{array}$} \\
\hline & $\begin{array}{l}\text { Quoted UK } \\
\text { companies }\end{array}$ & $\begin{array}{r}\text { Unquoted UK } \\
\text { companies }\end{array}$ & by UK residents & & & & & \\
\hline & F514 & F515 & F517 & F5 & $\mathrm{F} 7$ & $\mathrm{FL}$ & FA & BF90 \\
\hline $\begin{array}{l}2002 \\
2003 \\
2004 \\
2005 \\
2006\end{array}$ & $\begin{array}{r}\text { NHYB } \\
19.4 \\
20.8 \\
14.0 \\
11.2 \\
13.5\end{array}$ & $\begin{array}{c}\text { NHYC } \\
109.1 \\
108.0 \\
119.8 \\
124.5 \\
126.8\end{array}$ & $\begin{array}{r}\text { NROU } \\
- \\
- \\
- \\
- \\
-\end{array}$ & $\begin{array}{r}\text { NHXW } \\
128.5 \\
128.8 \\
133.8 \\
135.7 \\
140.2\end{array}$ & $\begin{array}{r}\text { NHYQ } \\
3.2 \\
3.3 \\
3.4 \\
3.5 \\
3.5\end{array}$ & $\begin{array}{rr} & \text { NHXD } \\
3 & 503.2 \\
3 & 990.6 \\
4 & 477.0 \\
5 & 219.0 \\
5 & 923.7\end{array}$ & $\begin{array}{rr} & \text { NHTZ } \\
3 & 578.1 \\
4 & 102.3 \\
4 & 634.4 \\
5 & 443.4 \\
6 & 232.5\end{array}$ & $\begin{array}{r}\text { NHSL } \\
74.9 \\
111.8 \\
157.4 \\
224.4 \\
308.8\end{array}$ \\
\hline $\begin{array}{l}2007 \\
2008\end{array}$ & $\begin{array}{l}5.0 \\
0.1\end{array}$ & $\begin{array}{l}133.3^{\dagger} \\
144.2^{\dagger}\end{array}$ & $\begin{array}{l}- \\
-\end{array}$ & $\begin{array}{l}138.3^{\dagger} \\
144.3^{\dagger}\end{array}$ & $\begin{array}{r}3.6 \\
22.5\end{array}$ & $\begin{array}{l}6529.1 \\
7546.2^{\dagger}\end{array}$ & $\begin{array}{l}6884.7 \\
7674.6^{\dagger}\end{array}$ & $\begin{array}{l}355.5 \\
128.4^{\dagger}\end{array}$ \\
\hline 2005 Q4 & 11.2 & 124.5 & - & 135.7 & 3.5 & 5219.0 & 5443.4 & 224.4 \\
\hline $\begin{array}{r}2006 \text { Q1 } \\
\text { Q2 } \\
\text { Q3 } \\
\text { Q4 }\end{array}$ & $\begin{array}{l}13.9 \\
12.8 \\
13.2 \\
13.5\end{array}$ & $\begin{array}{l}123.9 \\
125.8 \\
126.4 \\
126.8\end{array}$ & $\begin{array}{l}- \\
- \\
- \\
-\end{array}$ & $\begin{array}{l}137.8 \\
138.6 \\
139.6 \\
140.2\end{array}$ & $\begin{array}{l}3.7 \\
3.4 \\
3.5 \\
3.5\end{array}$ & $\begin{array}{l}5663.3 \\
5712.9 \\
5831.2 \\
5923.7\end{array}$ & 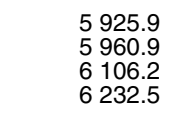 & $\begin{array}{l}262.6 \\
248.1 \\
275.1 \\
308.8\end{array}$ \\
\hline $\begin{array}{r}2008 \text { Q1 } \\
\text { Q2 } \\
\text { Q3 } \\
\text { Q4 }\end{array}$ & $\begin{array}{l}3.5 \\
1.8 \\
1.3 \\
0.1\end{array}$ & $\begin{array}{l}134.0 \\
135.9 \\
140.3^{\dagger} \\
144.2^{\dagger}\end{array}$ & $\begin{array}{l}- \\
- \\
- \\
-\end{array}$ & $\begin{array}{l}137.5 \\
137.7 \\
141.5 \\
144.3^{\dagger}\end{array}$ & $\begin{array}{r}3.9 \\
3.6 \\
17.5 \\
22.5\end{array}$ & $\begin{array}{ll}7 & 041.9^{\dagger} \\
6 & 606.9 \\
7 & 120.1 \\
7 & 546.2\end{array}$ & $\begin{array}{l}7326.6^{7} \\
6837.3^{\dagger} \\
7269.6 \\
7674.6\end{array}$ & $\begin{array}{l}284.7^{\dagger} \\
230.3 \\
149.5 \\
128.4\end{array}$ \\
\hline $\begin{array}{r}2009 \text { Q1 } \\
\text { Q2 }\end{array}$ & $\begin{array}{l}0.1 \\
0.1\end{array}$ & $\begin{array}{l}144.6 \\
144.3\end{array}$ & $\begin{array}{l}- \\
-\end{array}$ & $\begin{array}{l}144.7 \\
144.4\end{array}$ & $\begin{array}{l}22.7^{\dagger} \\
22.3\end{array}$ & $\begin{array}{ll}7 & 434.5 \\
7 & 036.1\end{array}$ & $\begin{array}{l}7444.5 \\
7108.6\end{array}$ & $\begin{array}{l}10.0 \\
72.5\end{array}$ \\
\hline
\end{tabular}

1 Monetary financial institutions

2 Money market instruments

Sources: National Statistics;

3 All loans secured on dwellings and all finance leasing are treated as long

term loans

4 Other than direct investment loans, loans secured on dwellings and loans for finance leasing 


\begin{tabular}{|c|c|c|c|c|c|c|c|c|c|c|c|c|c|c|c|c|}
\hline & \multicolumn{16}{|c|}{ Financial assets (BS) } \\
\hline & \multicolumn{5}{|c|}{ Currency and deposits } & \multicolumn{11}{|c|}{ Securities other than shares } \\
\hline & \multirow[b]{2}{*}{ Currency } & \multirow[b]{2}{*}{$\begin{array}{c}\text { Deposits } \\
\text { with UK } \\
\text { MFI's }^{1}\end{array}$} & \multirow{2}{*}{$\begin{array}{r}\text { Deposits } \\
\text { with } \\
\text { rest of } \\
\text { the } \\
\text { world } \\
\text { monetary } \\
\mathrm{fi}\end{array}$} & \multirow[b]{2}{*}{$\begin{array}{r}\text { Deposits } \\
\text { other } \\
\text { than } \\
\text { with } \\
\text { MFl's }\end{array}$} & \multirow[b]{2}{*}{ Total } & \multicolumn{5}{|c|}{ Short term MMl's ${ }^{2}$ issued } & \multicolumn{5}{|c|}{ Bonds issued } & \multirow[b]{2}{*}{ Total } \\
\hline & & & & & & $\begin{array}{r}\text { by UK } \\
\text { general } \\
\text { governm- } \\
\text { ent }\end{array}$ & $\begin{array}{l}\text { UK local } \\
\text { authori- } \\
\text { ty bills }\end{array}$ & $\begin{array}{r}\text { by UK } \\
\text { MFI's }\end{array}$ & $\begin{array}{r}\text { by other } \\
\text { UK } \\
\text { residen- } \\
\text { ts }\end{array}$ & $\begin{array}{l}\text { by the } \\
\text { rest of } \\
\text { the } \\
\text { world }\end{array}$ & $\begin{array}{r}\text { by UK } \\
\text { central } \\
\text { governm- } \\
\text { ent }\end{array}$ & $\begin{array}{r}\text { by UK } \\
\text { local } \\
\text { authori- } \\
\text { ties }\end{array}$ & $\begin{array}{r}\text { Medium } \\
\text { term by } \\
\text { MFl's }\end{array}$ & $\begin{array}{r}\text { Other by } \\
\text { UK } \\
\text { residen- } \\
\text { ts }\end{array}$ & $\begin{array}{l}\text { by the } \\
\text { rest of } \\
\text { the } \\
\text { world }\end{array}$ & \\
\hline & $\mathrm{F} 21$ & F221 & F229 & F29 & $\mathrm{F} 2$ & F3311 & F3312 & F3315 & F3316 & F3319 & $\mathrm{F} 3321$ & F3322 & F3325 & F3326 & F3329 & F3 \\
\hline $\begin{array}{l}2002 \\
2003 \\
2004 \\
2005 \\
2006\end{array}$ & $\begin{array}{r}\mathrm{NHYY} \\
0.6 \\
0.6 \\
0.6 \\
0.5 \\
0.5\end{array}$ & $\begin{array}{r}\mathrm{NHZA} \\
8.5 \\
7.6 \\
7.4 \\
8.7 \\
8.0\end{array}$ & $\begin{array}{r}\mathrm{NHZE} \\
0.4 \\
0.2 \\
0.3 \\
0.2 \\
0.3\end{array}$ & $\begin{array}{r}\mathrm{NHZF} \\
- \\
- \\
- \\
- \\
-\end{array}$ & $\begin{array}{r}\text { NHYX } \\
9.4 \\
8.5 \\
8.3 \\
9.5 \\
8.8\end{array}$ & $\begin{array}{r}\text { NHZI } \\
-\overline{1} \\
0.1 \\
0.1 \\
0.1 \\
0.1\end{array}$ & $\begin{array}{r}\mathrm{NHZM} \\
- \\
- \\
- \\
- \\
-\end{array}$ & $\begin{array}{r}\text { NHZN } \\
11.3 \\
14.2 \\
14.0 \\
14.8 \\
16.5\end{array}$ & $\begin{array}{r}\mathrm{NHZS} \\
0.4 \\
0.4 \\
0.3 \\
0.4 \\
0.4\end{array}$ & $\begin{array}{r}\mathrm{NHZT} \\
2.3 \\
2.2 \\
1.8 \\
1.6 \\
2.4\end{array}$ & $\begin{array}{r}\mathrm{NHZV} \\
0.2 \\
0.4 \\
1.0 \\
1.3 \\
1.0\end{array}$ & $\begin{array}{r}\mathrm{NHZY} \\
- \\
- \\
- \\
- \\
-\end{array}$ & $\begin{array}{r}\mathrm{NHZZ} \\
4.7 \\
6.1 \\
6.5 \\
7.6 \\
8.2\end{array}$ & $\begin{array}{r}\text { NIAC } \\
4.1 \\
5.3 \\
5.6 \\
6.3 \\
8.5\end{array}$ & $\begin{array}{r}\text { NIAD } \\
6.3 \\
4.8 \\
5.6 \\
5.9 \\
7.6\end{array}$ & $\begin{array}{r}\mathrm{NHZG} \\
29.3 \\
33.5 \\
34.9 \\
38.0 \\
44.8\end{array}$ \\
\hline 2005 Q4 & 0.5 & 8.7 & 0.2 & - & 9.5 & 0.1 & - & 14.8 & 0.4 & 1.6 & 1.3 & - & 7.6 & 6.3 & 5.9 & 38.0 \\
\hline $\begin{array}{r}2006 \text { Q1 } \\
\text { Q2 } \\
\text { Q3 } \\
\text { Q4 }\end{array}$ & $\begin{array}{l}0.4 \\
0.4 \\
0.4 \\
0.5\end{array}$ & $\begin{array}{l}7.8 \\
8.2 \\
6.0 \\
8.0\end{array}$ & $\begin{array}{l}0.1 \\
0.1 \\
0.1 \\
0.3\end{array}$ & $\begin{array}{l}- \\
- \\
- \\
-\end{array}$ & $\begin{array}{l}8.3 \\
8.7 \\
6.5 \\
8.8\end{array}$ & $\begin{array}{l}0.1 \\
0.1 \\
0.1 \\
0.1\end{array}$ & $\begin{array}{l}- \\
- \\
- \\
-\end{array}$ & $\begin{array}{l}12.6 \\
13.3 \\
15.3 \\
16.5\end{array}$ & $\begin{array}{l}0.3 \\
0.4 \\
0.4 \\
0.4\end{array}$ & $\begin{array}{l}1.3 \\
1.7 \\
2.0 \\
2.4\end{array}$ & $\begin{array}{l}1.4 \\
1.4 \\
1.1 \\
1.0\end{array}$ & $\begin{array}{l}- \\
- \\
- \\
-\end{array}$ & $\begin{array}{l}8.3 \\
8.1 \\
8.2 \\
8.2\end{array}$ & $\begin{array}{l}7.1 \\
7.6 \\
7.9 \\
8.5\end{array}$ & $\begin{array}{l}6.7 \\
7.2 \\
7.6 \\
7.6\end{array}$ & $\begin{array}{l}37.8 \\
39.7 \\
42.7 \\
44.8\end{array}$ \\
\hline $\begin{array}{r}2007 \text { Q1 } \\
\text { Q2 } \\
\text { Q3 } \\
\text { Q4 }\end{array}$ & $\begin{array}{l}0.4 \\
0.4 \\
0.4 \\
0.6\end{array}$ & $\begin{array}{r}6.0 \\
6.3 \\
11.5 \\
11.9\end{array}$ & $\begin{array}{l}0.1 \\
0.1 \\
0.7 \\
1.9\end{array}$ & $\begin{array}{l}- \\
- \\
- \\
-\end{array}$ & $\begin{array}{r}6.5 \\
6.8 \\
12.6 \\
14.3\end{array}$ & $\begin{array}{l}0.1 \\
0.1 \\
-\overline{7}\end{array}$ & $\begin{array}{l}- \\
- \\
- \\
-\end{array}$ & $\begin{array}{l}16.7 \\
18.3 \\
11.6 \\
17.9\end{array}$ & $\begin{array}{l}0.6 \\
0.4 \\
1.4 \\
0.4\end{array}$ & $\begin{array}{l}1.6 \\
1.7 \\
1.3 \\
1.5\end{array}$ & $\begin{array}{l}1.0 \\
1.1 \\
1.9 \\
5.0\end{array}$ & $\begin{array}{l}- \\
- \\
- \\
-\end{array}$ & $\begin{array}{l}8.7 \\
8.8 \\
8.5 \\
8.0\end{array}$ & $\begin{array}{l}8.5 \\
9.1 \\
9.0 \\
9.2\end{array}$ & $\begin{array}{r}8.0 \\
8.6 \\
9.2 \\
10.0\end{array}$ & $\begin{array}{l}45.2 \\
48.1 \\
43.0 \\
52.7\end{array}$ \\
\hline $\begin{array}{r}2008 \text { Q1 } \\
\text { Q2 } \\
\text { Q3 } \\
\text { Q4 }\end{array}$ & $\begin{array}{l}0.4 \\
0.3 \\
0.3 \\
0.4\end{array}$ & $\begin{array}{l}13.6 \\
14.0 \\
21.5 \\
21.0\end{array}$ & $\begin{array}{l}2.5 \\
2.1 \\
4.0 \\
1.3\end{array}$ & $\begin{array}{l}- \\
- \\
- \\
-\end{array}$ & $\begin{array}{l}16.5 \\
16.4 \\
25.9 \\
22.8\end{array}$ & $\begin{array}{l}0.2 \\
0.4 \\
0.4 \\
0.3\end{array}$ & $\begin{array}{l}- \\
- \\
- \\
-\end{array}$ & $\begin{array}{l}20.4 \\
23.2 \\
17.4 \\
11.9\end{array}$ & $\begin{array}{l}- \\
- \\
- \\
-\end{array}$ & $\begin{array}{l}2.3 \\
3.3 \\
3.0 \\
2.9\end{array}$ & $\begin{array}{l}4.9 \\
4.1 \\
5.0 \\
7.8\end{array}$ & $\begin{array}{l}- \\
- \\
- \\
-\end{array}$ & $\begin{array}{l}7.9 \\
7.8 \\
7.6 \\
8.4\end{array}$ & $\begin{array}{r}8.9^{\dagger} \\
8.7 \\
9.8 \\
10.7\end{array}$ & $\begin{array}{l}11.5^{\dagger} \\
11.2^{\dagger} \\
11.0 \\
12.6\end{array}$ & $\begin{array}{l}56.1^{\dagger} \\
58.6 \\
54.2 \\
54.7\end{array}$ \\
\hline $\begin{array}{r}2009 \text { Q1 } \\
\text { Q2 }\end{array}$ & $\begin{array}{l}0.3 \\
0.7\end{array}$ & $\begin{array}{l}19.5 \\
18.2\end{array}$ & $\begin{array}{l}0.2 \\
0.5\end{array}$ & $\begin{array}{l}- \\
-\end{array}$ & $\begin{array}{l}19.9 \\
19.4\end{array}$ & $\begin{array}{l}1.0 \\
2.2\end{array}$ & $\begin{array}{l}- \\
-\end{array}$ & $\begin{array}{r}11.2 \\
7.1\end{array}$ & $\begin{array}{l}- \\
-\end{array}$ & $\begin{array}{l}3.1 \\
3.0\end{array}$ & $\begin{array}{r}8.6 \\
10.0\end{array}$ & $\begin{array}{l}- \\
-\end{array}$ & $\begin{array}{l}9.2 \\
9.0\end{array}$ & $\begin{array}{l}10.6 \\
10.5\end{array}$ & $\begin{array}{l}12.9 \\
12.8\end{array}$ & $\begin{array}{l}56.6 \\
54.6\end{array}$ \\
\hline
\end{tabular}

Financial assets - continued (BS)

\begin{tabular}{|c|c|c|c|c|c|c|}
\hline & \multirow{2}{*}{\multicolumn{3}{|c|}{ Loans }} & \multirow{2}{*}{\multicolumn{2}{|c|}{ Shares and other equity }} & \\
\hline & & & & & & \multirow[b]{2}{*}{$\begin{array}{r}\text { Total financial } \\
\text { assets }\end{array}$} \\
\hline & $\begin{array}{c}\text { Short term loans } \\
\text { by UK MFl's }{ }^{3}\end{array}$ & $\begin{array}{l}\text { Long term loans } \\
\text { Secured on dwellings }\end{array}$ & Total & $\begin{array}{l}\text { Excl mutual funds' } \\
\text { shares } \\
\text { Unquoted UK shares }\end{array}$ & Total & \\
\hline & $\mathrm{F} 411$ & $\mathrm{~F} 422$ & $\mathrm{~F} 4$ & F515 & F5 & FA \\
\hline $\begin{array}{l}2002 \\
2003 \\
2004 \\
2005 \\
2006\end{array}$ & $\begin{array}{r}\text { NIAK } \\
21.8 \\
28.6 \\
32.3 \\
40.4 \\
49.8\end{array}$ & $\begin{array}{r}\text { NIAT } \\
123.6 \\
142.3 \\
165.4 \\
173.2 \\
189.7\end{array}$ & $\begin{array}{r}\mathrm{NIAI} \\
145.4 \\
170.9 \\
197.6 \\
213.7 \\
239.5\end{array}$ & $\begin{array}{r}\text { NIBG } \\
1.6 \\
1.5 \\
1.9 \\
1.9 \\
4.0\end{array}$ & $\begin{array}{r}\text { NIBA } \\
1.6 \\
1.5 \\
1.9 \\
1.9 \\
4.0\end{array}$ & $\begin{array}{l}\text { NIAH } \\
185.8 \\
214.3 \\
242.8 \\
263.0 \\
297.2\end{array}$ \\
\hline $\begin{array}{l}2007 \\
2008\end{array}$ & $\begin{array}{l}59.8^{\dagger} \\
85.8^{\dagger}\end{array}$ & $\begin{array}{l}202.7^{\dagger} \\
208.3^{\dagger}\end{array}$ & $\begin{array}{l}262.5^{\dagger} \\
294.1^{\dagger}\end{array}$ & $\begin{array}{l}2.5 \\
0.5\end{array}$ & $\begin{array}{l}2.5 \\
0.5\end{array}$ & $\begin{array}{l}331.9^{\dagger} \\
372.0^{\dagger}\end{array}$ \\
\hline 2005 Q4 & 40.4 & 173.2 & 213.7 & 1.9 & 1.9 & 263.0 \\
\hline $\begin{array}{r}2006 \text { Q1 } \\
\text { Q2 } \\
\text { Q3 } \\
\text { Q4 }\end{array}$ & $\begin{array}{l}42.7 \\
44.5 \\
46.9 \\
49.8\end{array}$ & $\begin{array}{l}175.8 \\
180.5 \\
186.4 \\
189.7\end{array}$ & $\begin{array}{l}218.5 \\
224.9 \\
233.3 \\
239.5\end{array}$ & $\begin{array}{l}2.0 \\
2.6 \\
2.1 \\
4.0\end{array}$ & $\begin{array}{l}2.0 \\
2.6 \\
2.1 \\
4.0\end{array}$ & $\begin{array}{l}266.6 \\
275.9 \\
284.6 \\
297.2\end{array}$ \\
\hline $\begin{array}{r}2007 \text { Q1 } \\
\text { Q2 } \\
\text { Q3 } \\
\text { Q4 }\end{array}$ & $\begin{array}{l}54.3 \\
51.2 \\
57.2 \\
59.8\end{array}$ & $\begin{array}{l}194.4 \\
198.2 \\
200.1 \\
202.7\end{array}$ & $\begin{array}{l}248.8 \\
249.4 \\
257.2 \\
262.5\end{array}$ & $\begin{array}{l}3.8 \\
4.2 \\
2.1 \\
2.5\end{array}$ & $\begin{array}{l}3.8 \\
4.2 \\
2.1 \\
2.5\end{array}$ & $\begin{array}{l}304.3 \\
308.4 \\
314.9 \\
331.9\end{array}$ \\
\hline $\begin{array}{r}2008 \text { Q1 } \\
\text { Q2 } \\
\text { Q3 } \\
\text { Q4 }\end{array}$ & $\begin{array}{l}61.9^{\dagger} \\
63.1 \\
77.3 \\
85.8\end{array}$ & $\begin{array}{l}206.9 \\
207.1 \\
207.2 \\
208.3^{\dagger}\end{array}$ & $\begin{array}{l}268.7^{\dagger} \\
270.3 \\
284.5 \\
294.1\end{array}$ & $\begin{array}{l}0.5 \\
0.5 \\
0.5 \\
0.5\end{array}$ & $\begin{array}{l}0.5 \\
0.5 \\
0.5 \\
0.5\end{array}$ & $\begin{array}{l}341.8^{\dagger} \\
345.7 \\
365.0 \\
372.0\end{array}$ \\
\hline $\begin{array}{r}2009 \text { Q1 } \\
\text { Q2 }\end{array}$ & $\begin{array}{l}85.4 \\
78.9\end{array}$ & $\begin{array}{l}206.1 \\
204.8\end{array}$ & $\begin{array}{l}291.5 \\
283.7\end{array}$ & $\begin{array}{l}0.5 \\
0.5\end{array}$ & $\begin{array}{l}0.5 \\
0.5\end{array}$ & $\begin{array}{l}368.5 \\
358.2\end{array}$ \\
\hline \multicolumn{5}{|c|}{$\begin{array}{l}1 \text { Monetary financial institutions } \\
2 \text { Money market instruments } \\
3 \text { All loans secured on dwellings and all finance leasing are treated as long } \\
\text { term loans } \\
4 \text { Other than with direct investment loans, loans secured on dwellings and } \\
\text { loans for finance leasing. }\end{array}$} & Sourc & $\begin{array}{l}\text { ional Statistics; } \\
\text { ank of England }\end{array}$ \\
\hline
\end{tabular}




\begin{tabular}{|c|c|c|c|c|c|c|}
\hline & \multicolumn{6}{|c|}{ Financial liabilities (BS) } \\
\hline & \multicolumn{2}{|c|}{ Currency and deposits } & \multicolumn{4}{|c|}{ Securities other than shares } \\
\hline & \multirow[b]{2}{*}{ Deposits with UK MFIs ${ }^{1}$} & \multirow[b]{2}{*}{ Total } & \multirow{2}{*}{$\begin{array}{l}\text { Short term MMl's } \\
\text { issued } \\
\text { by UK MFI's }\end{array}$} & \multicolumn{2}{|c|}{ Bonds issued } & \multirow[b]{2}{*}{ Total } \\
\hline & & & & medium term by MFI's & other by UK residents & \\
\hline & $\mathrm{F} 22$ & $\mathrm{~F} 2$ & F3315 & F3325 & F3326 & F3 \\
\hline $\begin{array}{l}2002 \\
2003 \\
2004 \\
2005 \\
2006\end{array}$ & $\begin{array}{l}\text { NICE } \\
155.2 \\
168.7 \\
181.9 \\
201.6 \\
219.4\end{array}$ & $\begin{array}{l}\text { NICB } \\
155.2 \\
168.7 \\
181.9 \\
201.6 \\
219.4\end{array}$ & $\begin{array}{r}\text { NICR } \\
9.5 \\
17.5 \\
19.3 \\
15.6 \\
24.5\end{array}$ & $\begin{array}{r}\text { NIDD } \\
8.9 \\
12.6 \\
17.4 \\
23.6 \\
23.1\end{array}$ & $\begin{array}{r}\text { NIDG } \\
5.7 \\
7.5 \\
9.5 \\
9.8 \\
11.4\end{array}$ & $\begin{array}{r}\text { NICK } \\
24.1 \\
37.7 \\
46.2 \\
49.0 \\
59.0\end{array}$ \\
\hline $\begin{array}{l}2007 \\
2008\end{array}$ & $\begin{array}{l}248.8 \\
267.4\end{array}$ & $\begin{array}{l}248.8 \\
267.4\end{array}$ & $\begin{array}{l}20.6^{\dagger} \\
11.4^{\dagger}\end{array}$ & $\begin{array}{l}24.5 \\
23.9\end{array}$ & $\begin{array}{r}14.0 \\
7.1\end{array}$ & $\begin{array}{l}59.0^{\dagger} \\
42.4^{\dagger}\end{array}$ \\
\hline $\begin{array}{r}2006 \text { Q1 } \\
\text { Q2 } \\
\text { Q3 } \\
\text { Q4 }\end{array}$ & $\begin{array}{l}206.6 \\
212.6 \\
216.9 \\
219.4\end{array}$ & $\begin{array}{l}206.6 \\
212.6 \\
216.9 \\
219.4\end{array}$ & $\begin{array}{l}15.8 \\
16.2 \\
20.5 \\
24.5\end{array}$ & $\begin{array}{l}23.3 \\
22.7 \\
22.6 \\
23.1\end{array}$ & $\begin{array}{r}9.9 \\
10.1 \\
10.1 \\
11.4\end{array}$ & $\begin{array}{l}49.0 \\
49.0 \\
53.2 \\
59.0\end{array}$ \\
\hline $\begin{array}{r}2007 \text { Q1 } \\
\text { Q2 } \\
\text { Q3 } \\
\text { Q4 }\end{array}$ & $\begin{array}{l}223.2 \\
230.8 \\
239.3 \\
248.8\end{array}$ & $\begin{array}{l}223.2 \\
230.8 \\
239.3 \\
248.8\end{array}$ & $\begin{array}{l}23.3 \\
23.1 \\
21.6 \\
20.6\end{array}$ & $\begin{array}{l}23.4 \\
24.7 \\
25.4 \\
24.5\end{array}$ & $\begin{array}{l}13.0 \\
13.1 \\
13.1 \\
14.0\end{array}$ & $\begin{array}{l}59.7 \\
60.8 \\
60.1 \\
59.0\end{array}$ \\
\hline $\begin{array}{r}2008 \text { Q1 } \\
\text { Q2 } \\
\text { Q3 } \\
\text { Q4 }\end{array}$ & $\begin{array}{l}259.4^{\dagger} \\
268.2 \\
268.6 \\
267.4\end{array}$ & $\begin{array}{l}259.4^{\dagger} \\
268.2 \\
268.6 \\
267.4\end{array}$ & $\begin{array}{l}19.3^{\dagger} \\
16.5^{\dagger} \\
17.3^{-4} \\
11.4\end{array}$ & $\begin{array}{l}24.3 \\
23.3 \\
20.5 \\
23.9\end{array}$ & $\begin{array}{r}12.3 \\
12.4 \\
11.7 \\
7.1\end{array}$ & $\begin{array}{l}55.9 \\
52.1^{\dagger} \\
49.5 \\
42.4\end{array}$ \\
\hline \multirow[t]{3}{*}{$\begin{array}{r}2009 \text { Q1 } \\
\text { Q2 }\end{array}$} & $\begin{array}{l}269.2 \\
264.8\end{array}$ & $\begin{array}{l}269.2 \\
264.8\end{array}$ & $\begin{array}{l}15.2 \\
18.8\end{array}$ & $\begin{array}{l}21.7 \\
18.8\end{array}$ & $\begin{array}{l}7.6 \\
9.0\end{array}$ & $\begin{array}{l}44.5 \\
46.6\end{array}$ \\
\hline & \multicolumn{4}{|c|}{ Financial liabilities - continued (BS) } & \multicolumn{2}{|c|}{$\begin{array}{c}\text { Net financial } \\
\text { assets/liabilities }\end{array}$} \\
\hline & Unquoted UK shares & $\begin{array}{r}\text { Total shares and } \\
\text { other equity }\end{array}$ & $\begin{array}{l}\text { Other accounts } \\
\text { receivable/payable }\end{array}$ & $\begin{array}{r}\text { Total financial } \\
\text { liabilities }\end{array}$ & $\begin{array}{r}\text { Total financial } \\
\text { assets }\end{array}$ & $\begin{array}{l}\text { assets } \\
\text { ilities(-) }\end{array}$ \\
\hline & F515 & F5 & $\mathrm{F} 7$ & $\mathrm{FL}$ & $\mathrm{FA}$ & BF90 \\
\hline $\begin{array}{l}2002 \\
2003 \\
2004 \\
2005 \\
2006\end{array}$ & $\begin{array}{r}\text { NIEE } \\
- \\
- \\
- \\
- \\
-\end{array}$ & $\begin{array}{r}\text { NIEK } \\
- \\
- \\
- \\
- \\
-\end{array}$ & $\begin{array}{r}\text { NIEY } \\
0.7 \\
0.7 \\
1.6 \\
2.3 \\
2.7\end{array}$ & $\begin{array}{c}\text { NIDL } \\
179.9 \\
207.2 \\
229.7 \\
252.9 \\
281.1\end{array}$ & $\begin{array}{l}\text { NIAH } \\
185.8 \\
214.3 \\
242.8 \\
263.0 \\
297.2\end{array}$ & $\begin{array}{r}\text { NHYT } \\
5.9 \\
7.2 \\
13.1 \\
10.2 \\
16.1\end{array}$ \\
\hline $\begin{array}{l}2007 \\
2008\end{array}$ & $\begin{array}{l}- \\
-\end{array}$ & $\begin{array}{l}- \\
-\end{array}$ & $\begin{array}{l}4.0 \\
4.0\end{array}$ & $\begin{array}{l}311.8 \\
313.8^{\dagger}\end{array}$ & $\begin{array}{l}331.9 \\
372.0^{\dagger}\end{array}$ & $\begin{array}{l}20.1^{\dagger} \\
58.2^{\dagger}\end{array}$ \\
\hline 2005 Q4 & - & - & 2.3 & 252.9 & 263.0 & 10.2 \\
\hline $\begin{array}{r}2006 \text { Q1 } \\
\text { Q2 } \\
\text { Q3 } \\
\text { Q4 }\end{array}$ & $\begin{array}{l}- \\
- \\
- \\
-\end{array}$ & $\begin{array}{l}- \\
- \\
- \\
-\end{array}$ & $\begin{array}{l}1.3 \\
1.3 \\
1.9 \\
2.7\end{array}$ & $\begin{array}{l}256.8 \\
262.9 \\
272.0 \\
281.1\end{array}$ & $\begin{array}{l}266.6 \\
275.9 \\
284.6 \\
297.2\end{array}$ & $\begin{array}{r}9.7 \\
13.0 \\
12.7 \\
16.1\end{array}$ \\
\hline $\begin{array}{r}2007 \text { Q1 } \\
\text { Q2 } \\
\text { Q3 } \\
\text { Q4 }\end{array}$ & $\begin{array}{l}- \\
- \\
- \\
-\end{array}$ & $\begin{array}{l}- \\
- \\
- \\
-\end{array}$ & $\begin{array}{l}1.6 \\
2.1 \\
3.1 \\
4.0\end{array}$ & $\begin{array}{l}284.6 \\
293.7 \\
302.5 \\
311.8\end{array}$ & $\begin{array}{l}304.3 \\
308.4 \\
314.9 \\
331.9\end{array}$ & $\begin{array}{l}19.7 \\
14.8 \\
12.4 \\
20.1\end{array}$ \\
\hline $\begin{array}{r}2008 \text { Q1 } \\
\text { Q2 } \\
\text { Q3 } \\
\text { Q4 }\end{array}$ & $\begin{array}{l}- \\
- \\
- \\
-\end{array}$ & $\begin{array}{l}- \\
- \\
- \\
-\end{array}$ & $\begin{array}{l}2.7 \\
2.7 \\
3.6 \\
4.0\end{array}$ & $\begin{array}{l}318.0^{\dagger} \\
323.0 \\
321.7 \\
313.8\end{array}$ & $\begin{array}{l}341.8^{\dagger} \\
345.7 \\
365.0 \\
372.0\end{array}$ & $\begin{array}{l}23.8^{\dagger} \\
22.7 \\
43.3 \\
58.2\end{array}$ \\
\hline $\begin{array}{r}2009 \text { Q1 } \\
\text { Q2 }\end{array}$ & $\begin{array}{l}- \\
-\end{array}$ & $\begin{array}{l}- \\
-\end{array}$ & $\begin{array}{l}1.8^{\dagger} \\
0.8\end{array}$ & $\begin{array}{l}315.5 \\
312.2\end{array}$ & $\begin{array}{l}368.5 \\
358.2\end{array}$ & $\begin{array}{l}53.0 \\
46.0\end{array}$ \\
\hline
\end{tabular}




\begin{tabular}{|c|c|c|c|c|c|c|c|c|c|c|c|c|c|c|c|c|c|c|}
\hline & \multicolumn{18}{|c|}{ Financial assets } \\
\hline & \multicolumn{7}{|c|}{ Currency and deposits } & \multicolumn{11}{|c|}{ Securities other than shares } \\
\hline & \multirow[b]{2}{*}{$\begin{array}{r}\text { Curren- } \\
\text { cy }\end{array}$} & \multirow[b]{2}{*}{$\begin{array}{r}\text { Sterli- } \\
\text { ng Bank } \\
\text { deposi- } \\
\text { ts }\end{array}$} & \multirow[b]{2}{*}{$\begin{array}{r}\text { Foreign } \\
\text { curren- } \\
\text { cy Bank } \\
\text { deposi- } \\
\text { ts }\end{array}$} & \multirow[b]{2}{*}{$\begin{array}{r}\text { Sterli- } \\
\text { ng } \\
\text { B.socs } \\
\text { deposi- } \\
\text { ts }\end{array}$} & \multirow[b]{2}{*}{$\begin{array}{r}\text { Deposi- } \\
\text { ts with } \\
\text { rest of } \\
\text { the } \\
\text { world } \\
\text { MFl's }\end{array}$} & \multirow[b]{2}{*}{$\begin{array}{r}\text { Deposi- } \\
\text { ts } \\
\text { other } \\
\text { than } \\
\text { with } \\
\text { MFl's }\end{array}$} & \multirow[b]{2}{*}{ Total } & \multicolumn{5}{|c|}{ Short term MMl's ${ }^{2}$ issued } & \multicolumn{5}{|c|}{ Bonds issued } & \multirow[b]{2}{*}{ Total } \\
\hline & & & & & & & & $\begin{array}{r}\text { by UK } \\
\text { general } \\
\text { govern- } \\
\text { ment }\end{array}$ & $\begin{array}{r}\text { UK } \\
\text { local } \\
\text { author- } \\
\text { ity } \\
\text { bills }\end{array}$ & $\begin{array}{l}\text { by UK } \\
\text { MFI's }\end{array}$ & $\begin{array}{r}\text { by } \\
\text { other } \\
\text { UK } \\
\text { reside- } \\
\text { nts }\end{array}$ & $\begin{array}{l}\text { by the } \\
\text { rest of } \\
\text { the } \\
\text { world }\end{array}$ & $\begin{array}{r}\text { by UK } \\
\text { central } \\
\text { govern- } \\
\text { ment }\end{array}$ & $\begin{array}{r}\text { by UK } \\
\text { local } \\
\text { author- } \\
\text { ities }\end{array}$ & $\begin{array}{r}\text { Medium } \\
\text { term by } \\
\text { MFl's }\end{array}$ & $\begin{array}{r}\text { Other } \\
\text { by UK } \\
\text { reside- } \\
\text { nts }\end{array}$ & $\begin{array}{r}\text { Other } \\
\text { by the } \\
\text { rest of } \\
\text { the } \\
\text { world }\end{array}$ & \\
\hline & $\mathrm{F} 21$ & $\mathrm{~F} 2211$ & F2212 & F2213 & $\mathrm{F} 229$ & F29 & $\mathrm{F} 2$ & F3311 & F3312 F & F3315 & F3316 & F3319 & F3321 & F3322 & F3325 & F3326 & F3329 & F3 \\
\hline $\begin{array}{l}2002 \\
2003 \\
2004 \\
2005 \\
2006\end{array}$ & $\begin{array}{r}\text { NLPM } \\
0.1 \\
0.1 \\
0.1 \\
0.1 \\
0.1\end{array}$ & $\begin{array}{l}\text { NLPP } \\
117.2 \\
117.1 \\
140.7 \\
209.8 \\
279.9\end{array}$ & $\begin{array}{l}\text { NLPQ } \\
103.7 \\
128.2 \\
140.9 \\
173.1 \\
200.9\end{array}$ & $\begin{array}{r}\text { NLPR } \\
1.2 \\
2.5 \\
2.7 \\
2.9 \\
3.1\end{array}$ & $\begin{array}{l}\text { NLPS } \\
248.5 \\
296.2 \\
324.8 \\
506.9 \\
514.8\end{array}$ & $\begin{array}{r}\text { NLPT } \\
1.8 \\
0.8 \\
2.2 \\
3.5 \\
1.5\end{array}$ & $\begin{array}{r}\text { NLPL } \\
472.5 \\
545.0 \\
611.3 \\
896.3 \\
1000.2\end{array}$ & $\begin{array}{r}\text { NLPW } \\
0.9 \\
3.1 \\
4.0 \\
1.9 \\
2.9\end{array}$ & $\begin{array}{r}\text { NLQA } \\
- \\
- \\
- \\
- \\
-\end{array}$ & $\begin{array}{r}\text { NLQB } \\
27.1 \\
25.6 \\
22.3 \\
23.1 \\
22.9\end{array}$ & $\begin{array}{r}\text { NLQG } \\
0.9 \\
0.7 \\
1.2 \\
1.0 \\
1.6\end{array}$ & $\begin{array}{r}\text { NLQH } \\
5.6 \\
7.8 \\
8.5 \\
7.5 \\
10.5\end{array}$ & $\begin{array}{r}\text { NLQJ } \\
27.3 \\
31.3 \\
31.1 \\
44.9 \\
63.4\end{array}$ & $\begin{array}{r}\text { NLQM } \\
- \\
- \\
- \\
- \\
-\end{array}$ & $\begin{array}{r}\text { NLQN } \\
5.0 \\
8.2 \\
10.2 \\
13.8 \\
16.6\end{array}$ & $\begin{array}{r}\text { NLQQ } \\
56.0 \\
69.7 \\
79.5 \\
89.9 \\
96.9\end{array}$ & $\begin{array}{r}\text { NLQR } \\
38.8 \\
50.3 \\
71.8 \\
95.4 \\
71.5\end{array}$ & $\begin{array}{l}\text { NLPU } \\
161.5 \\
196.7 \\
228.7 \\
277.4 \\
286.4\end{array}$ \\
\hline $\begin{array}{l}2007 \\
2008\end{array}$ & $\begin{array}{l}0.1 \\
0.1\end{array}$ & $\begin{array}{l}334.9^{\dagger} \\
534.7^{\dagger}\end{array}$ & $\begin{array}{l}265.3^{\dagger} \\
332.0^{\dagger}\end{array}$ & $\begin{array}{l}6.0 \\
6.2\end{array}$ & $\begin{array}{l}570.7 \\
379.5^{\dagger}\end{array}$ & $\begin{array}{r}3.7 \\
11.5\end{array}$ & $\begin{array}{l}1180.7 \\
1264.0^{\dagger}\end{array}$ & $\begin{array}{l}4.1 \\
5.7^{\dagger}\end{array}$ & $\begin{array}{l}- \\
-\end{array}$ & $\begin{array}{l}28.7 \\
38.2^{\dagger}\end{array}$ & $\begin{array}{l}2.3 \\
0.2\end{array}$ & $\begin{array}{l}7.6^{\dagger} \\
4.6^{\dagger}\end{array}$ & $\begin{array}{r}84.0^{\dagger} \\
137.6^{\dagger}\end{array}$ & $\begin{array}{l}- \\
-\end{array}$ & $\begin{array}{l}23.2 \\
28.3\end{array}$ & $\begin{array}{r}99.5^{\dagger} \\
114.3^{\dagger}\end{array}$ & $\begin{array}{r}53.0 \\
+\quad 188.2^{\dagger}\end{array}$ & $\begin{array}{l}302.4 \\
516.9^{\dagger}\end{array}$ \\
\hline 2005 Q4 & 0.1 & 209.8 & 173.1 & 2.9 & 506.9 & 3.5 & 896.3 & 1.9 & - & 23.1 & 1.0 & 7.5 & 44.9 & - & 13.8 & 89.9 & 95.4 & 277.4 \\
\hline $\begin{array}{r}2006 \text { Q1 } \\
\text { Q2 } \\
\text { Q3 } \\
\text { Q4 }\end{array}$ & $\begin{array}{l}0.1 \\
0.1 \\
0.1 \\
0.1\end{array}$ & $\begin{array}{l}221.2 \\
242.1 \\
264.5 \\
279.9\end{array}$ & $\begin{array}{l}200.7 \\
181.3 \\
198.3 \\
200.9\end{array}$ & $\begin{array}{l}3.4 \\
3.3 \\
3.4 \\
3.1\end{array}$ & $\begin{array}{l}588.6 \\
538.6 \\
501.0 \\
514.8\end{array}$ & $\begin{array}{l}1.5 \\
1.7 \\
0.8 \\
1.5\end{array}$ & $\begin{array}{r}1015.6 \\
967.0 \\
968.1 \\
1000.2\end{array}$ & $\begin{array}{l}3.1 \\
4.4 \\
3.0 \\
2.9\end{array}$ & $\begin{array}{l}- \\
- \\
- \\
-\end{array}$ & $\begin{array}{l}21.6 \\
27.6 \\
26.6 \\
22.9\end{array}$ & $\begin{array}{l}0.9 \\
1.2 \\
1.8 \\
1.6\end{array}$ & $\begin{array}{r}9.9 \\
11.1 \\
9.3 \\
10.5\end{array}$ & $\begin{array}{l}57.9 \\
59.4 \\
59.4 \\
63.4\end{array}$ & $\begin{array}{l}- \\
- \\
- \\
-\end{array}$ & $\begin{array}{l}15.7 \\
15.0 \\
15.8 \\
16.6\end{array}$ & $\begin{array}{l}87.8 \\
87.1 \\
86.4 \\
96.9\end{array}$ & $\begin{array}{l}91.1 \\
75.4 \\
64.5 \\
71.5\end{array}$ & $\begin{array}{l}288.1 \\
281.2 \\
266.8 \\
286.4\end{array}$ \\
\hline $\begin{array}{r}2007 \text { Q1 } \\
\text { Q2 } \\
\text { Q3 } \\
\text { Q4 }\end{array}$ & $\begin{array}{l}0.1 \\
0.1 \\
0.1 \\
0.1\end{array}$ & $\begin{array}{l}304.9 \\
323.8 \\
345.2 \\
334.9\end{array}$ & $\begin{array}{l}214.5 \\
242.0 \\
238.8 \\
265.3\end{array}$ & $\begin{array}{l}3.5 \\
4.3 \\
4.9 \\
6.0\end{array}$ & $\begin{array}{l}580.1 \\
552.9 \\
554.6 \\
570.7\end{array}$ & $\begin{array}{l}1.2 \\
2.7 \\
3.5 \\
3.7\end{array}$ & $\begin{array}{ll}1 & 104.2 \\
1 & 125.8 \\
1 & 147.0 \\
1 & 180.7\end{array}$ & $\begin{array}{l}2.8 \\
3.5 \\
5.2 \\
4.1\end{array}$ & $\begin{array}{l}- \\
- \\
- \\
-\end{array}$ & $\begin{array}{l}24.8 \\
26.1 \\
29.8 \\
28.7\end{array}$ & $\begin{array}{l}1.5 \\
1.9 \\
2.3 \\
2.3\end{array}$ & $\begin{array}{r}10.7 \\
12.4 \\
8.8 \\
7.6\end{array}$ & $\begin{array}{l}73.5 \\
69.3 \\
79.0 \\
84.0\end{array}$ & $\begin{array}{l}- \\
- \\
- \\
-\end{array}$ & $\begin{array}{l}17.7 \\
20.1 \\
22.8 \\
23.2\end{array}$ & $\begin{array}{l}96.6 \\
97.6 \\
96.2 \\
99.5\end{array}$ & $\begin{array}{l}70.4 \\
64.5 \\
52.3 \\
53.0\end{array}$ & $\begin{array}{l}298.0 \\
295.3 \\
296.4 \\
302.4\end{array}$ \\
\hline $\begin{array}{r}2008 \text { Q1 } \\
\text { Q2 } \\
\text { Q3 } \\
\text { Q4 }\end{array}$ & $\begin{array}{l}0.1 \\
0.1 \\
0.1 \\
0.1\end{array}$ & $\begin{array}{l}362.0^{\dagger} \\
400.2 \\
456.1 \\
534.7\end{array}$ & $\begin{array}{l}318.8 \\
286.4 \\
278.0^{\dagger} \\
332.0^{\dagger}\end{array}$ & $\begin{array}{l}6.1 \\
6.1 \\
6.2 \\
6.2\end{array}$ & $\begin{array}{l}617.1 \\
506.5 \\
491.3^{\dagger} \\
379.5^{\dagger}\end{array}$ & $\begin{array}{r}5.1 \\
11.7 \\
7.5 \\
11.5\end{array}$ & $\begin{array}{ll}1 & 309.2^{\dagger} \\
1 & 211.1 \\
1 & 239.2 \\
1 & 264.0\end{array}$ & $\begin{array}{l}3.7 \\
2.6^{\dagger} \\
5.2 \\
5.7\end{array}$ & $\begin{array}{l}- \\
- \\
- \\
-\end{array}$ & $\begin{array}{l}31.1^{\dagger} \\
35.8 \\
29.4 \\
38.2\end{array}$ & $\begin{array}{l}2.5 \\
1.9 \\
2.0 \\
0.2\end{array}$ & $\begin{array}{l}7.5 \\
6.5 \\
4.3^{\dagger} \\
4.6^{\dagger}\end{array}$ & $\begin{array}{c}89.9 \\
89.2 \\
97.7^{\dagger} \\
137.6\end{array}$ & $\begin{array}{l}- \\
- \\
- \\
-\end{array}$ & $\begin{array}{l}23.2 \\
23.9 \\
24.1 \\
28.3\end{array}$ & $\begin{array}{c}98.8 \\
103.3 \\
109.0^{\dagger} \\
114.3\end{array}$ & $\begin{array}{r}80.5 \\
+112.7 \\
138.1 \\
188.2^{\dagger}\end{array}$ & $\begin{array}{r}337.1^{\dagger} \\
376.0 \\
409.9 \\
516.9\end{array}$ \\
\hline $\begin{array}{r}2009 \text { Q1 } \\
\text { Q2 }\end{array}$ & $\begin{array}{l}0.1 \\
0.1\end{array}$ & $\begin{array}{l}611.3 \\
574.5\end{array}$ & $\begin{array}{l}300.9 \\
295.2\end{array}$ & $\begin{array}{l}6.3 \\
6.4\end{array}$ & $\begin{array}{l}341.4 \\
349.9\end{array}$ & $\begin{array}{l}24.3 \\
14.5\end{array}$ & $\begin{array}{l}1284.3 \\
1240.6\end{array}$ & $\begin{array}{l}11.2 \\
12.4\end{array}$ & $\begin{array}{l}- \\
-\end{array}$ & $\begin{array}{l}43.1 \\
38.8\end{array}$ & $\begin{array}{l}0.4 \\
0.2\end{array}$ & $\begin{array}{l}5.6 \\
6.2\end{array}$ & $\begin{array}{l}127.9 \\
104.4\end{array}$ & $\begin{array}{l}- \\
-\end{array}$ & $\begin{array}{l}28.4^{\dagger} \\
28.9\end{array}$ & $\begin{array}{r}114.7 \\
120.3\end{array}$ & $\begin{array}{l}220.5 \\
229.6\end{array}$ & $\begin{array}{l}551.8 \\
540.8\end{array}$ \\
\hline
\end{tabular}

Financial assets - continued

\begin{tabular}{|c|c|}
\hline Loans & Shares and other equity \\
\hline
\end{tabular}

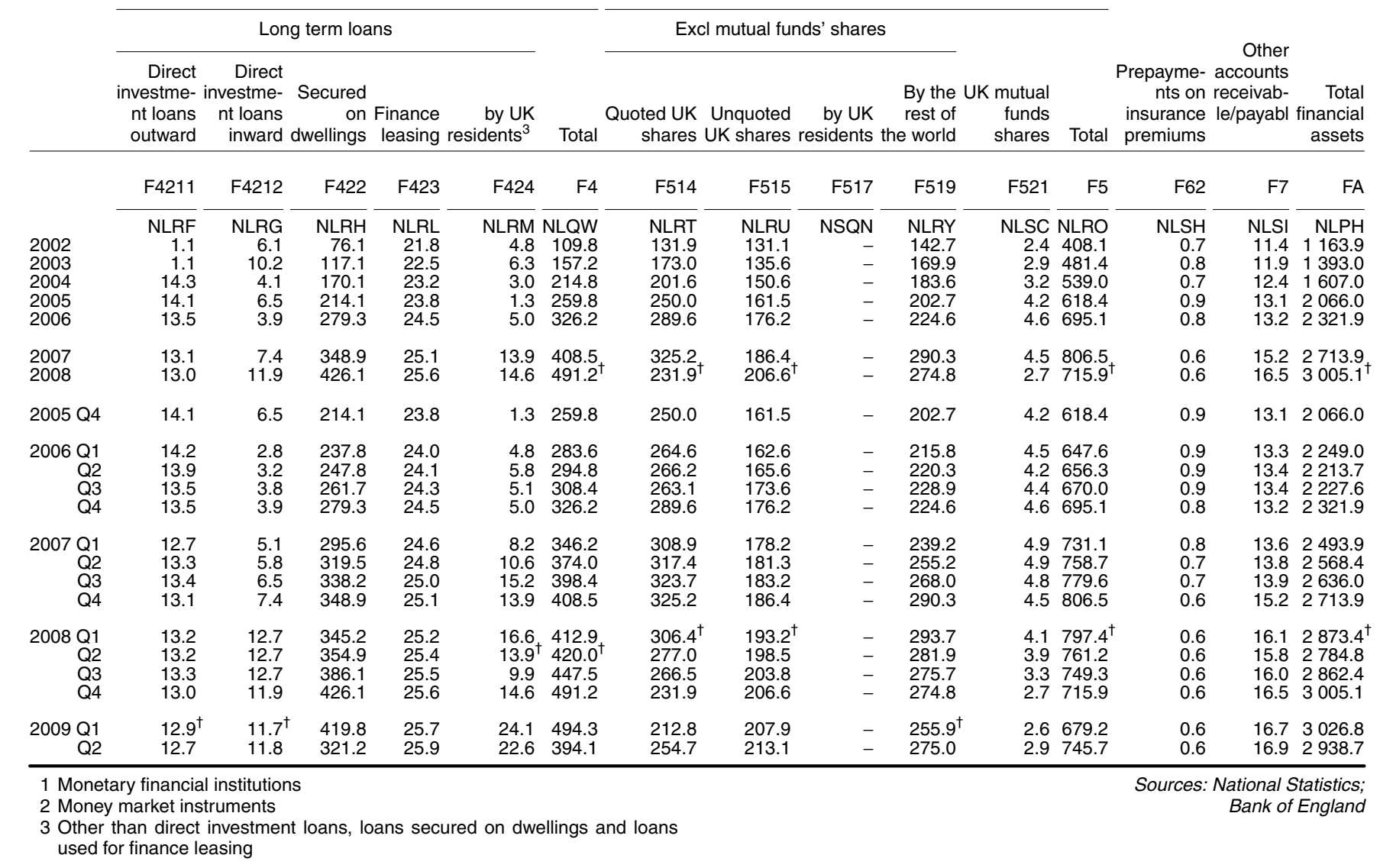




\begin{tabular}{|c|c|c|c|c|c|c|c|c|c|c|c|c|c|c|}
\hline & \multicolumn{14}{|c|}{ Financial liabilities } \\
\hline & \multirow[b]{3}{*}{$\begin{array}{r}\text { Currency } \\
\text { and } \\
\text { Deposits }\end{array}$} & \multicolumn{3}{|c|}{ Securities other than shares } & \multicolumn{10}{|c|}{ Loans } \\
\hline & & $\begin{array}{l}\text { Short } \\
\text { term } \\
\text { MMl's }^{1} \\
\text { issued }\end{array}$ & $\begin{array}{l}\text { Bonds } \\
\text { issued }\end{array}$ & & \multicolumn{4}{|c|}{ Short term loans } & \multicolumn{5}{|c|}{ Long term loans } & \multirow[b]{2}{*}{ Total } \\
\hline & & $\begin{array}{r}\text { by other } \\
\text { UK } \\
\text { residents }\end{array}$ & $\begin{array}{r}\text { other by } \\
\text { UK } \\
\text { residents }\end{array}$ & Total & $\begin{array}{r}\text { Sterling } \\
\text { loans By } \\
\text { UK MFl's }{ }^{2,3}\end{array}$ & $\begin{array}{l}\text { Foreign } \\
\text { currency } \\
\text { loans by } \\
\text { UK MFIs }\end{array}$ & $\begin{array}{l}\text { Sterling } \\
\text { loans by } \\
\text { B.socs }\end{array}$ & $\begin{array}{c}\text { By rest } \\
\text { of the } \\
\text { world } \\
\text { MFIs }\end{array}$ & $\begin{array}{r}\text { Direct } \\
\text { investme- } \\
\text { nt loans } \\
\text { outward }\end{array}$ & $\begin{array}{r}\text { Direct } \\
\text { investme- } \\
\text { nt loans } \\
\text { inward }\end{array}$ & $\begin{array}{r}\text { Finance } \\
\text { leasing }\end{array}$ & $\begin{array}{r}\text { by UK } \\
\text { residents }\end{array}$ & $\begin{array}{r}\text { Other by } \\
\text { the rest } \\
\text { of the } \\
\text { world }\end{array}$ & \\
\hline & AF2 & F3316 & F3326 & F3 & F4111 & F4112 & F4113 & F419 & F4211 & F4212 & F423 & F424 & F429 & $\mathrm{F} 4$ \\
\hline $\begin{array}{l}2002 \\
2003 \\
2004 \\
2005 \\
2006\end{array}$ & $\begin{array}{r}\text { NJUF } \\
0.8 \\
0.3 \\
0.3 \\
1.8 \\
1.6\end{array}$ & $\begin{array}{r}\text { NLTK } \\
21.1 \\
19.4 \\
20.2 \\
26.2 \\
42.8\end{array}$ & $\begin{array}{l}\text { NLTU } \\
189.4 \\
243.6 \\
333.3 \\
430.4 \\
512.8\end{array}$ & $\begin{array}{l}\text { NLSY } \\
210.5 \\
263.1 \\
353.5 \\
456.6 \\
555.6\end{array}$ & $\begin{array}{l}\text { NLUD } \\
173.8 \\
193.5 \\
229.3 \\
259.1 \\
302.3\end{array}$ & $\begin{array}{l}\text { NLUE } \\
163.5 \\
202.9 \\
226.7 \\
253.1 \\
289.4\end{array}$ & $\begin{array}{r}\text { NLUF } \\
11.3 \\
16.4 \\
19.8 \\
26.5 \\
32.8\end{array}$ & $\begin{array}{l}\text { NLUG } \\
357.8 \\
384.3 \\
446.2 \\
670.3 \\
602.3\end{array}$ & $\begin{array}{r}\text { NLUJ } \\
1.2 \\
1.2 \\
20.6 \\
20.6 \\
20.2\end{array}$ & $\begin{array}{r}\text { NLUK } \\
24.4 \\
28.8 \\
24.0 \\
18.0 \\
18.2\end{array}$ & $\begin{array}{r}\text { NLUP } \\
1.4 \\
1.5 \\
1.6 \\
1.7 \\
1.7\end{array}$ & $\begin{array}{r}\text { NLUQ } \\
14.6 \\
15.8 \\
19.7 \\
26.2 \\
30.9\end{array}$ & $\begin{array}{r}\text { NLUR } \\
0.5 \\
0.5 \\
0.5 \\
0.5 \\
0.5\end{array}$ & $\begin{array}{r}\text { NLUA } \\
748.6 \\
844.8 \\
988.3 \\
1275.8 \\
1298.4\end{array}$ \\
\hline $\begin{array}{l}2007 \\
2008\end{array}$ & $\begin{array}{r}5.9 \\
17.3\end{array}$ & $\begin{array}{l}45.4^{\dagger} \\
72.5^{\dagger}\end{array}$ & $\begin{array}{l}627.8^{\dagger} \\
679.4^{\dagger}\end{array}$ & $\begin{array}{l}673.2 \\
751.9^{\dagger}\end{array}$ & $\begin{array}{l}441.9^{\dagger} \\
588.2^{\dagger}\end{array}$ & $\begin{array}{l}382.7 \\
454.0^{\dagger}\end{array}$ & $\begin{array}{l}41.2^{\dagger} \\
66.1^{\dagger}\end{array}$ & $\begin{array}{l}682.0^{\dagger} \\
540.1^{\dagger}\end{array}$ & $\begin{array}{l}20.5 \\
20.4\end{array}$ & $\begin{array}{l}22.2 \\
24.1\end{array}$ & $\begin{array}{l}1.8 \\
1.9\end{array}$ & $\begin{array}{l}30.0 \\
31.2\end{array}$ & $\begin{array}{l}0.5 \\
0.5\end{array}$ & $\begin{array}{l}1622.9 \\
1726.4^{\dagger}\end{array}$ \\
\hline 2005 Q4 & 1.8 & 26.2 & 430.4 & 456.6 & 259.1 & 253.1 & 26.5 & 670.3 & 20.6 & 18.0 & 1.7 & 26.2 & 0.5 & 1275.8 \\
\hline $\begin{array}{r}2006 \text { Q1 } \\
\text { Q2 } \\
\text { Q3 } \\
\text { Q4 }\end{array}$ & $\begin{array}{l}3.4 \\
1.7 \\
1.6 \\
1.6\end{array}$ & $\begin{array}{l}33.6 \\
44.8 \\
41.0 \\
42.8\end{array}$ & $\begin{array}{l}456.8 \\
468.7 \\
496.6 \\
512.8\end{array}$ & $\begin{array}{l}490.4 \\
513.5 \\
537.6 \\
555.6\end{array}$ & $\begin{array}{l}274.7 \\
292.3 \\
304.1 \\
302.3\end{array}$ & $\begin{array}{l}298.8 \\
284.1 \\
270.0 \\
289.4\end{array}$ & $\begin{array}{l}28.2 \\
29.1 \\
30.9 \\
32.8\end{array}$ & $\begin{array}{l}711.0 \\
639.4 \\
599.2 \\
602.3\end{array}$ & $\begin{array}{l}20.3 \\
20.3 \\
19.9 \\
20.2\end{array}$ & $\begin{array}{l}19.2 \\
19.5 \\
18.9 \\
18.2\end{array}$ & $\begin{array}{l}1.7 \\
1.7 \\
1.7 \\
1.7\end{array}$ & $\begin{array}{l}29.2 \\
27.2 \\
28.4 \\
30.9\end{array}$ & $\begin{array}{l}0.5 \\
0.5 \\
0.5 \\
0.5\end{array}$ & $\begin{array}{ll}1 & 383.6 \\
1 & 314.1 \\
1 & 273.6 \\
1 & 298.4\end{array}$ \\
\hline $\begin{array}{r}2007 \text { Q1 } \\
\text { Q2 } \\
\text { Q3 } \\
\text { Q4 }\end{array}$ & $\begin{array}{l}2.7 \\
1.8 \\
2.2 \\
5.9\end{array}$ & $\begin{array}{l}41.3 \\
47.6 \\
54.0 \\
45.4\end{array}$ & $\begin{array}{l}552.6 \\
581.5 \\
596.7 \\
627.8\end{array}$ & $\begin{array}{l}593.9 \\
629.1 \\
650.7 \\
673.2\end{array}$ & $\begin{array}{l}379.5 \\
392.8 \\
439.5 \\
441.9\end{array}$ & $\begin{array}{l}330.5 \\
355.0 \\
359.2 \\
382.7\end{array}$ & $\begin{array}{l}37.0 \\
33.5 \\
38.9 \\
41.2\end{array}$ & $\begin{array}{l}685.6 \\
722.9 \\
714.5 \\
682.0\end{array}$ & $\begin{array}{l}20.6 \\
20.4 \\
20.5 \\
20.5\end{array}$ & $\begin{array}{l}19.0 \\
19.2 \\
14.5 \\
22.2\end{array}$ & $\begin{array}{l}1.8 \\
1.8 \\
1.8 \\
1.8\end{array}$ & $\begin{array}{l}32.8 \\
31.0 \\
30.9 \\
30.0\end{array}$ & $\begin{array}{l}0.5 \\
0.5 \\
0.5 \\
0.5\end{array}$ & $\begin{array}{ll}1 & 507.1 \\
1 & 577.1 \\
1 & 620.3 \\
1 & 622.9\end{array}$ \\
\hline $\begin{array}{r}2008 \text { Q1 } \\
\text { Q2 } \\
\text { Q3 } \\
\text { Q4 }\end{array}$ & $\begin{array}{r}8.0 \\
5.9 \\
8.7 \\
17.3\end{array}$ & $\begin{array}{l}71.9^{\dagger} \\
67.7 \\
65.4 \\
72.5\end{array}$ & $\begin{array}{l}616.2^{\dagger} \\
633.1 \\
620.8 \\
679.4\end{array}$ & $\begin{array}{l}688.1^{\dagger} \\
700.8 \\
686.2 \\
751.9\end{array}$ & $\begin{array}{l}468.0^{\dagger} \\
499.6 \\
540.4 \\
588.2\end{array}$ & $\begin{array}{l}453.7 \\
385.5 \\
398.7 \\
454.0^{\dagger}\end{array}$ & $\begin{array}{l}42.3^{\dagger} \\
43.1 \\
57.0 \\
66.1\end{array}$ & $\begin{array}{l}792.9^{\dagger} \\
682.4 \\
623.4 \\
540.1\end{array}$ & $\begin{array}{l}20.6 \\
20.4 \\
20.7 \\
20.4\end{array}$ & $\begin{array}{l}25.3 \\
24.7 \\
24.3 \\
24.1\end{array}$ & $\begin{array}{l}1.8 \\
1.9 \\
1.9 \\
1.9\end{array}$ & $\begin{array}{l}35.4 \\
31.2 \\
33.8 \\
31.2\end{array}$ & $\begin{array}{l}0.5 \\
0.5 \\
0.5 \\
0.5\end{array}$ & $\begin{array}{l}1840.5^{\dagger} \\
1689.2 \\
1700.6 \\
1726.4\end{array}$ \\
\hline $\begin{array}{r}2009 \text { Q1 } \\
\text { Q2 }\end{array}$ & $\begin{array}{l}33.3 \\
23.6\end{array}$ & $\begin{array}{l}73.3 \\
62.3\end{array}$ & $\begin{array}{l}600.7 \\
629.4\end{array}$ & $\begin{array}{l}674.0 \\
691.7\end{array}$ & $\begin{array}{l}628.7 \\
533.1\end{array}$ & $\begin{array}{l}407.1 \\
362.8\end{array}$ & $\begin{array}{l}65.4 \\
60.1\end{array}$ & $\begin{array}{l}472.3 \\
460.5\end{array}$ & $\begin{array}{l}21.0 \\
20.7\end{array}$ & $\begin{array}{l}23.8 \\
23.7\end{array}$ & $\begin{array}{l}1.9 \\
1.9\end{array}$ & $\begin{array}{l}24.2^{\dagger} \\
23.0\end{array}$ & $\begin{array}{l}0.5 \\
0.5\end{array}$ & $\begin{array}{l}1644.8 \\
1486.3\end{array}$ \\
\hline
\end{tabular}

\begin{tabular}{|c|c|c|c|c|c|c|c|c|c|}
\hline & \multicolumn{5}{|c|}{ Shares and other equity } & \multirow{3}{*}{$\begin{array}{r}\text { Other accounts } \\
\text { receivable/pay- } \\
\text { able }\end{array}$} & \multirow[b]{3}{*}{$\begin{array}{r}\text { Total financial } \\
\text { liabilities }\end{array}$} & \multirow[b]{3}{*}{$\begin{array}{r}\text { Total financial } \\
\text { assets }\end{array}$} & \multirow{3}{*}{$\begin{array}{r}\text { Net assets } \\
(+) / \text { liabilitie- } \\
\text { s(-) }\end{array}$} \\
\hline & \multicolumn{3}{|c|}{ Excl mutual funds' shares } & \multirow[b]{2}{*}{$\begin{array}{r}\text { UK mutual funds } \\
\text { shares }\end{array}$} & \multirow[b]{2}{*}{ Total } & & & & \\
\hline & $\begin{array}{l}\text { Quoted UK } \\
\text { companies }\end{array}$ & $\begin{array}{r}\text { Unquoted UK } \\
\text { companies }\end{array}$ & by UK residents & & & & & & \\
\hline & F514 & F515 & $\mathrm{F} 517$ & $\mathrm{~F} 521$ & F5 & $\mathrm{F} 7$ & FL & FA & BF90 \\
\hline $\begin{array}{l}2002 \\
2003 \\
2004 \\
2005 \\
2006\end{array}$ & $\begin{array}{l}\text { NLUX } \\
215.6 \\
274.2 \\
303.7 \\
341.0 \\
399.2\end{array}$ & $\begin{array}{l}\text { NLUY } \\
135.9 \\
155.3 \\
176.0 \\
204.0 \\
245.0\end{array}$ & $\begin{array}{r}\text { NSQO } \\
- \\
- \\
- \\
- \\
-\end{array}$ & $\begin{array}{l}\text { NLVG } \\
215.8 \\
266.3 \\
303.9 \\
385.0 \\
452.4\end{array}$ & $\begin{array}{r}\text { NLUS } \\
567.3 \\
695.8 \\
783.6 \\
930.0 \\
1096.6\end{array}$ & $\begin{array}{r}\text { NLVM } \\
1.8 \\
2.3 \\
2.2 \\
2.1 \\
2.5\end{array}$ & $\begin{array}{rl} & \text { NLSL } \\
1 & 528.9 \\
1 & 806.3 \\
2 & 127.9 \\
2 & 666.3 \\
2 & 954.7\end{array}$ & $\begin{array}{r}\text { NLPH } \\
1163.9 \\
1393.0 \\
1607.0 \\
2066.0 \\
2321.9\end{array}$ & $\begin{array}{r}\text { NYOF } \\
-365.0 \\
-413.3 \\
-520.9 \\
-600.3 \\
-632.8\end{array}$ \\
\hline $\begin{array}{l}2007 \\
2008\end{array}$ & $\begin{array}{l}353.4 \\
177.1\end{array}$ & $\begin{array}{l}244.7^{\dagger} \\
326.6^{\dagger}\end{array}$ & $\begin{array}{l}- \\
-\end{array}$ & $\begin{array}{l}507.3^{\dagger} \\
382.3^{\dagger}\end{array}$ & $\begin{array}{r}1105.4 \\
885.9^{\dagger}\end{array}$ & $\begin{array}{l}0.5 \\
1.0\end{array}$ & $\begin{array}{l}3408.0^{\dagger} \\
3382.5^{\dagger}\end{array}$ & $\begin{array}{l}2713.9 \\
3005.1^{\dagger}\end{array}$ & $\begin{array}{l}-694.2^{\dagger} \\
-377.5^{\dagger}\end{array}$ \\
\hline 2005 Q4 & 341.0 & 204.0 & - & 385.0 & 930.0 & 2.1 & 2666.3 & 2066.0 & -600.3 \\
\hline $\begin{array}{r}2006 \text { Q1 } \\
\text { Q2 } \\
\text { Q3 } \\
\text { Q4 }\end{array}$ & $\begin{array}{l}369.0 \\
355.6 \\
379.8 \\
399.2\end{array}$ & $\begin{array}{l}226.3 \\
235.8 \\
237.4 \\
245.0\end{array}$ & $\begin{array}{l}- \\
- \\
- \\
-\end{array}$ & $\begin{array}{l}424.0 \\
409.5 \\
426.1 \\
452.4\end{array}$ & $\begin{array}{ll}1 & 019.4 \\
1 & 001.0 \\
1 & 043.3 \\
1 & 096.6\end{array}$ & $\begin{array}{l}2.2 \\
2.2 \\
2.3 \\
2.5\end{array}$ & $\begin{array}{l}2898.9 \\
2832.4 \\
2858.5 \\
2954.7\end{array}$ & $\begin{array}{l}2249.0 \\
2213.7 \\
2227.6 \\
2321.9\end{array}$ & $\begin{array}{l}-649.8 \\
-618.7 \\
-630.8 \\
-632.8\end{array}$ \\
\hline $\begin{array}{r}2007 \text { Q1 } \\
\text { Q2 } \\
\text { Q3 } \\
\text { Q4 }\end{array}$ & $\begin{array}{l}392.9 \\
392.3 \\
370.8 \\
353.4\end{array}$ & $\begin{array}{l}215.2 \\
227.0 \\
228.9 \\
244.7\end{array}$ & $\begin{array}{l}- \\
- \\
- \\
-\end{array}$ & $\begin{array}{l}493.0 \\
508.8 \\
511.1 \\
507.3\end{array}$ & $\begin{array}{ll}1 & 101.1 \\
1 & 128.1 \\
1 & 110.8 \\
1 & 105.4\end{array}$ & $\begin{array}{l}2.3 \\
1.8 \\
1.1 \\
0.5\end{array}$ & $\begin{array}{l}3207.1 \\
3337.9 \\
3385.2 \\
3408.0\end{array}$ & $\begin{array}{l}2493.9 \\
2568.4 \\
2636.0 \\
2713.9\end{array}$ & $\begin{array}{l}-713.2 \\
-769.5 \\
-749.2 \\
-694.2\end{array}$ \\
\hline $\begin{array}{r}2008 \text { Q1 } \\
\text { Q2 } \\
\text { Q3 } \\
\text { Q4 }\end{array}$ & $\begin{array}{l}299.7 \\
258.3 \\
255.9 \\
177.1\end{array}$ & $\begin{array}{l}248.2^{\dagger} \\
279.5 \\
297.8 \\
326.6\end{array}$ & $\begin{array}{l}- \\
- \\
- \\
-\end{array}$ & $\begin{array}{l}468.9 \\
458.9 \\
407.4^{\dagger} \\
382.3\end{array}$ & $\begin{array}{c}1016.7 \\
996.6^{\dagger} \\
961.2 \\
885.9\end{array}$ & $\begin{array}{l}0.4 \\
0.4 \\
0.6 \\
1.0\end{array}$ & $\begin{array}{l}3553.7^{\dagger} \\
3393.0 \\
3357.3 \\
3382.5\end{array}$ & $\begin{array}{l}2873.4^{\dagger} \\
2784.8 \\
2862.4 \\
3005.1\end{array}$ & $\begin{array}{l}-680.3^{\dagger} \\
-608.2 \\
-494.9 \\
-377.5\end{array}$ \\
\hline $\begin{array}{r}2009 \text { Q1 } \\
\text { Q2 }\end{array}$ & $\begin{array}{l}151.1 \\
230.2\end{array}$ & $\begin{array}{l}339.1 \\
360.9\end{array}$ & $\begin{array}{l}- \\
-\end{array}$ & $\begin{array}{l}368.5 \\
411.3\end{array}$ & $\begin{array}{r}858.7 \\
1002.4\end{array}$ & $\begin{array}{l}1.3 \\
1.4\end{array}$ & $\begin{array}{l}32212.1 \\
3205.4\end{array}$ & $\begin{array}{l}3026.8 \\
2938.7\end{array}$ & $\begin{array}{l}-185.3 \\
-266.8\end{array}$ \\
\hline
\end{tabular}

1 Money market instruments

2 All loans secured on dwellings and all finance leasing are treated as long

Sources: National Statistics; term loans

3 Monetary financial institutions

4 Other than direct investment loans, loans secured on dwellings and loans for finance leasing. 


\begin{tabular}{|c|c|c|c|c|c|c|c|c|c|c|c|c|c|c|c|c|}
\hline & \multicolumn{16}{|c|}{ Financial assets } \\
\hline & \multicolumn{6}{|c|}{ Currency and deposits } & \multicolumn{10}{|c|}{ Securities other than shares } \\
\hline & \multirow[b]{2}{*}{$\begin{array}{r}\text { Sterling } \\
\text { Bank } \\
\text { deposits }\end{array}$} & \multirow[b]{2}{*}{$\begin{array}{r}F C \\
\text { deposits } \\
\text { with UK } \\
\text { banks }\end{array}$} & \multirow{2}{*}{$\begin{array}{r}\text { Sterling } \\
\text { building } \\
\text { societi- } \\
\text { es } \\
\text { deposits }\end{array}$} & \multirow{2}{*}{$\begin{array}{r}\text { Deposits } \\
\text { with } \\
\text { rest of } \\
\text { the } \\
\text { world } \\
\text { MFl's }\end{array}$} & \multirow{2}{*}{$\begin{array}{r}\text { Deposits } \\
\text { other } \\
\text { than } \\
\text { with } \\
\text { MFl's }\end{array}$} & \multirow[b]{2}{*}{ Total } & \multicolumn{4}{|c|}{ Short term MMl's ${ }^{2}$ issued } & \multicolumn{5}{|c|}{ Bonds issued } & \multirow[b]{2}{*}{ Total } \\
\hline & & & & & & & $\begin{array}{r}\text { by UK } \\
\text { general } \\
\text { governm- } \\
\text { ent }\end{array}$ & $\begin{array}{r}\text { by UK } \\
\text { MFI's }\end{array}$ & $\begin{array}{r}\text { by other } \\
\text { UK } \\
\text { residen- } \\
\text { ts }\end{array}$ & $\begin{array}{l}\text { by the } \\
\text { rest of } \\
\text { the } \\
\text { world }\end{array}$ & $\begin{array}{r}\text { by UK } \\
\text { central } \\
\text { governm- } \\
\text { ent }\end{array}$ & $\begin{array}{r}\text { by UK } \\
\text { local } \\
\text { authori- } \\
\text { ties }\end{array}$ & $\begin{array}{r}\text { Medium } \\
\text { term by } \\
\text { MFI's }\end{array}$ & $\begin{array}{r}\text { Other by } \\
\text { UK } \\
\text { residen- } \\
\text { ts }\end{array}$ & $\begin{array}{r}\text { by the } \\
\text { rest of } \\
\text { the } \\
\text { world }\end{array}$ & \\
\hline & F2211 & F2212 & F2213 & F229 & F29 & $\mathrm{F} 2$ & F3311 & F3315 & F3316 & F3319 & F3321 & F3322 & F3325 & F3326 & F3329 & F3 \\
\hline $\begin{array}{l}2002 \\
2003 \\
2004 \\
2005 \\
2006\end{array}$ & $\begin{array}{l}\text { NIYH } \\
53.1 \\
49.5 \\
55.3 \\
51.5 \\
56.5\end{array}$ & $\begin{array}{r}\text { IE2Y } \\
3.3 \\
4.8 \\
5.6 \\
4.8 \\
7.2\end{array}$ & $\begin{array}{r}\text { NIYJ } \\
3.4 \\
3.1 \\
3.5 \\
3.4 \\
3.9\end{array}$ & $\begin{array}{r}\text { NIYK } \\
8.1 \\
15.5 \\
25.3 \\
33.9 \\
37.4\end{array}$ & $\begin{array}{r}\text { NIYL } \\
- \\
- \\
- \\
- \\
-\end{array}$ & $\begin{array}{r}\text { NIYD } \\
67.9 \\
72.9 \\
89.7 \\
93.7 \\
104.9\end{array}$ & $\begin{array}{r}\text { NIYO } \\
0.8 \\
0.5 \\
1.0 \\
0.4 \\
0.7\end{array}$ & $\begin{array}{l}\text { NIYT } \\
21.5 \\
24.6 \\
26.6 \\
24.9 \\
29.1\end{array}$ & $\begin{array}{r}\text { NIYY } \\
3.3 \\
3.4 \\
1.9 \\
5.5 \\
3.6\end{array}$ & $\begin{array}{r}\text { NIYZ } \\
1.4 \\
1.5 \\
2.1 \\
3.5 \\
3.0\end{array}$ & $\begin{array}{l}\text { NIZB } \\
184.3 \\
202.4 \\
224.6 \\
231.0 \\
241.1\end{array}$ & $\begin{array}{r}\text { NIZE } \\
0.5 \\
0.4 \\
0.3 \\
0.4 \\
0.5\end{array}$ & $\begin{array}{l}\text { NIZF } \\
13.7 \\
23.1 \\
29.1 \\
39.5 \\
47.9\end{array}$ & $\begin{array}{r}\text { NIZI } \\
171.1 \\
174.2 \\
175.4 \\
186.4 \\
178.2\end{array}$ & $\begin{array}{r}\text { NIZJ } \\
109.3 \\
118.6 \\
142.3 \\
168.3 \\
212.8\end{array}$ & $\begin{array}{l}\text { NIYM } \\
505.8 \\
548.7 \\
603.4 \\
660.1 \\
717.0\end{array}$ \\
\hline $\begin{array}{l}2007 \\
2008\end{array}$ & $\begin{array}{l}71.6^{\dagger} \\
72.8^{\dagger}\end{array}$ & $\begin{array}{l}8.0^{\dagger} \\
7.3^{\dagger}\end{array}$ & $\begin{array}{l}4.7 \\
2.9\end{array}$ & $\begin{array}{l}46.0^{\dagger} \\
56.5^{\dagger}\end{array}$ & $\begin{array}{l}- \\
-\end{array}$ & $\begin{array}{l}130.4^{\dagger} \\
139.5^{\dagger}\end{array}$ & $\begin{array}{l}0.6^{\dagger} \\
1.0^{\dagger}\end{array}$ & $\begin{array}{l}30.6^{\dagger} \\
25.3^{\dagger}\end{array}$ & $\begin{array}{l}5.9 \\
4.7\end{array}$ & $\begin{array}{l}3.1^{\dagger} \\
5.1^{\dagger}\end{array}$ & $\begin{array}{l}240.7^{\dagger} \\
242.8^{\dagger}\end{array}$ & $\begin{array}{l}0.3 \\
0.2\end{array}$ & $\begin{array}{l}67.5 \\
82.6\end{array}$ & $\begin{array}{l}162.4^{\dagger} \\
148.0^{\dagger}\end{array}$ & $\begin{array}{l}258.0^{\dagger} \\
363.3^{\dagger}\end{array}$ & $\begin{array}{l}769.1 \\
872.9^{\dagger}\end{array}$ \\
\hline 2005 Q4 & 51.5 & 4.8 & 3.4 & 33.9 & - & 93.7 & 0.4 & 24.9 & 5.5 & 3.5 & 231.0 & 0.4 & 39.5 & 186.4 & 168.3 & 660.1 \\
\hline $\begin{array}{r}2006 \text { Q1 } \\
\text { Q2 } \\
\text { Q3 } \\
\text { Q4 }\end{array}$ & $\begin{array}{l}54.5 \\
57.9 \\
57.7 \\
56.5\end{array}$ & $\begin{array}{l}6.2 \\
6.1 \\
5.6 \\
7.2\end{array}$ & $\begin{array}{l}3.8 \\
3.8 \\
4.1 \\
3.9\end{array}$ & $\begin{array}{l}36.5 \\
35.4 \\
38.2 \\
37.4\end{array}$ & $\begin{array}{l}- \\
- \\
- \\
-\end{array}$ & $\begin{array}{l}101.1 \\
103.2 \\
105.6 \\
104.9\end{array}$ & $\begin{array}{l}0.6 \\
0.6 \\
0.6 \\
0.7\end{array}$ & $\begin{array}{l}27.4 \\
26.5 \\
27.6 \\
29.1\end{array}$ & $\begin{array}{l}3.2 \\
5.2 \\
5.9 \\
3.6\end{array}$ & $\begin{array}{l}2.2 \\
2.9 \\
3.2 \\
3.0\end{array}$ & $\begin{array}{l}241.9 \\
247.5 \\
252.3 \\
241.1\end{array}$ & $\begin{array}{l}0.5 \\
0.5 \\
0.5 \\
0.5\end{array}$ & $\begin{array}{l}45.4 \\
43.3 \\
45.4 \\
47.9\end{array}$ & $\begin{array}{l}179.3 \\
178.6 \\
183.6 \\
178.2\end{array}$ & $\begin{array}{l}181.6 \\
189.7 \\
203.9 \\
212.8\end{array}$ & $\begin{array}{l}682.0 \\
694.8 \\
723.0 \\
717.0\end{array}$ \\
\hline $\begin{array}{r}2007 \text { Q1 } \\
\text { Q2 } \\
\text { Q3 } \\
\text { Q4 }\end{array}$ & $\begin{array}{l}54.2 \\
58.7 \\
59.6 \\
71.6\end{array}$ & $\begin{array}{l}6.5 \\
6.2 \\
7.1 \\
8.0\end{array}$ & $\begin{array}{l}4.0 \\
3.9 \\
4.2 \\
4.7\end{array}$ & $\begin{array}{l}40.5 \\
43.2 \\
44.3 \\
46.0\end{array}$ & $\begin{array}{l}- \\
- \\
- \\
-\end{array}$ & $\begin{array}{l}105.3 \\
112.1 \\
115.3 \\
130.4\end{array}$ & $\begin{array}{l}0.4 \\
0.3 \\
0.3 \\
0.6\end{array}$ & $\begin{array}{l}30.5 \\
27.6 \\
31.8 \\
30.6\end{array}$ & $\begin{array}{l}3.7 \\
5.5 \\
7.3 \\
5.9\end{array}$ & $\begin{array}{l}2.8 \\
2.8 \\
3.6 \\
3.1\end{array}$ & $\begin{array}{l}246.1 \\
228.8 \\
235.7 \\
240.7\end{array}$ & $\begin{array}{l}0.5 \\
0.4 \\
0.3 \\
0.3\end{array}$ & $\begin{array}{l}51.1 \\
58.3 \\
66.4 \\
67.5\end{array}$ & $\begin{array}{l}176.5 \\
165.3 \\
160.6 \\
162.4\end{array}$ & $\begin{array}{l}221.2 \\
229.4 \\
239.5 \\
258.0\end{array}$ & $\begin{array}{l}732.7 \\
718.3 \\
745.5 \\
769.1\end{array}$ \\
\hline $\begin{array}{r}2008 \text { Q1 } \\
\text { Q2 } \\
\text { Q3 } \\
\text { Q4 }\end{array}$ & $\begin{array}{l}74.8^{\dagger} \\
74.9 \\
75.2 \\
72.8\end{array}$ & $\begin{array}{l}9.7 \\
9.5 \\
8.1 \\
7.3^{\dagger}\end{array}$ & $\begin{array}{l}4.7 \\
4.0 \\
4.0 \\
2.9\end{array}$ & $\begin{array}{l}55.2^{\dagger} \\
52.4 \\
53.6 \\
56.5\end{array}$ & $\begin{array}{l}- \\
- \\
- \\
-\end{array}$ & $\begin{array}{l}144.5^{\dagger} \\
140.9 \\
140.9 \\
139.5\end{array}$ & $\begin{array}{l}0.8 \\
0.7^{\dagger} \\
2.1 \\
1.0\end{array}$ & $\begin{array}{l}29.7^{\dagger} \\
27.9 \\
29.8 \\
25.3\end{array}$ & $\begin{array}{l}4.9 \\
5.4 \\
6.1 \\
4.7\end{array}$ & $\begin{array}{l}4.2 \\
4.8 \\
5.2 \\
5.1^{\dagger}\end{array}$ & $\begin{array}{l}238.4^{\dagger} \\
235.1 \\
227.0 \\
242.8\end{array}$ & $\begin{array}{l}0.3 \\
0.3 \\
0.3 \\
0.2\end{array}$ & $\begin{array}{l}67.5 \\
69.6 \\
70.2 \\
82.6\end{array}$ & $\begin{array}{l}159.7^{\dagger} \\
159.9 \\
152.4 \\
148.0\end{array}$ & $\begin{array}{l}265.8 \\
274.7 \\
303.0^{\dagger} \\
363.3\end{array}$ & $\begin{array}{r}771.3^{\dagger} \\
+778.5 \\
796.0 \\
872.9\end{array}$ \\
\hline $\begin{array}{r}2009 \text { Q1 } \\
\text { Q2 }\end{array}$ & $\begin{array}{l}68.8 \\
69.3\end{array}$ & $\begin{array}{l}7.0 \\
7.0\end{array}$ & $\begin{array}{l}3.1^{\dagger} \\
2.4^{-}\end{array}$ & $\begin{array}{l}49.8 \\
46.2\end{array}$ & $\begin{array}{l}- \\
-\end{array}$ & $\begin{array}{l}128.7 \\
124.9\end{array}$ & $\begin{array}{l}1.2 \\
2.2\end{array}$ & $\begin{array}{l}22.8 \\
23.1\end{array}$ & $\begin{array}{l}4.1 \\
4.6\end{array}$ & $\begin{array}{l}4.4 \\
4.3\end{array}$ & $\begin{array}{l}230.6 \\
232.2\end{array}$ & $\begin{array}{l}0.1^{\dagger} \\
0.2\end{array}$ & $\begin{array}{l}82.8^{\dagger} \\
84.2\end{array}$ & $\begin{array}{l}151.2 \\
168.0\end{array}$ & $\begin{array}{l}366.6 \\
353.8\end{array}$ & $\begin{array}{l}863.8 \\
872.7\end{array}$ \\
\hline
\end{tabular}

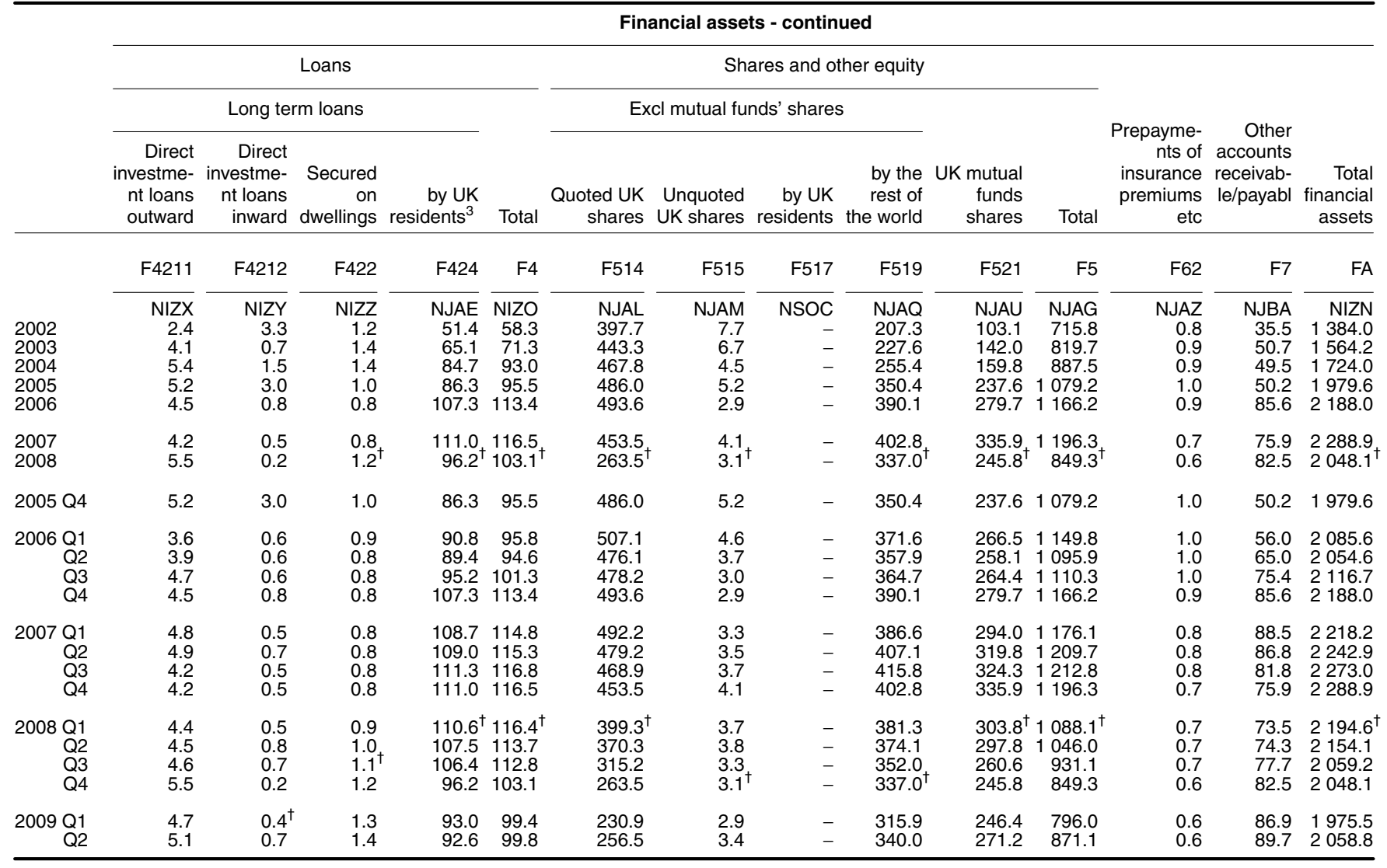

1 Monetary financial institutions

2 Money market instruments
3 Other than direct investment loans, loans secured on dwellings and loans

Sources: National Statistics;

Bank of England

secured on dwellings and loans for finance leasing 


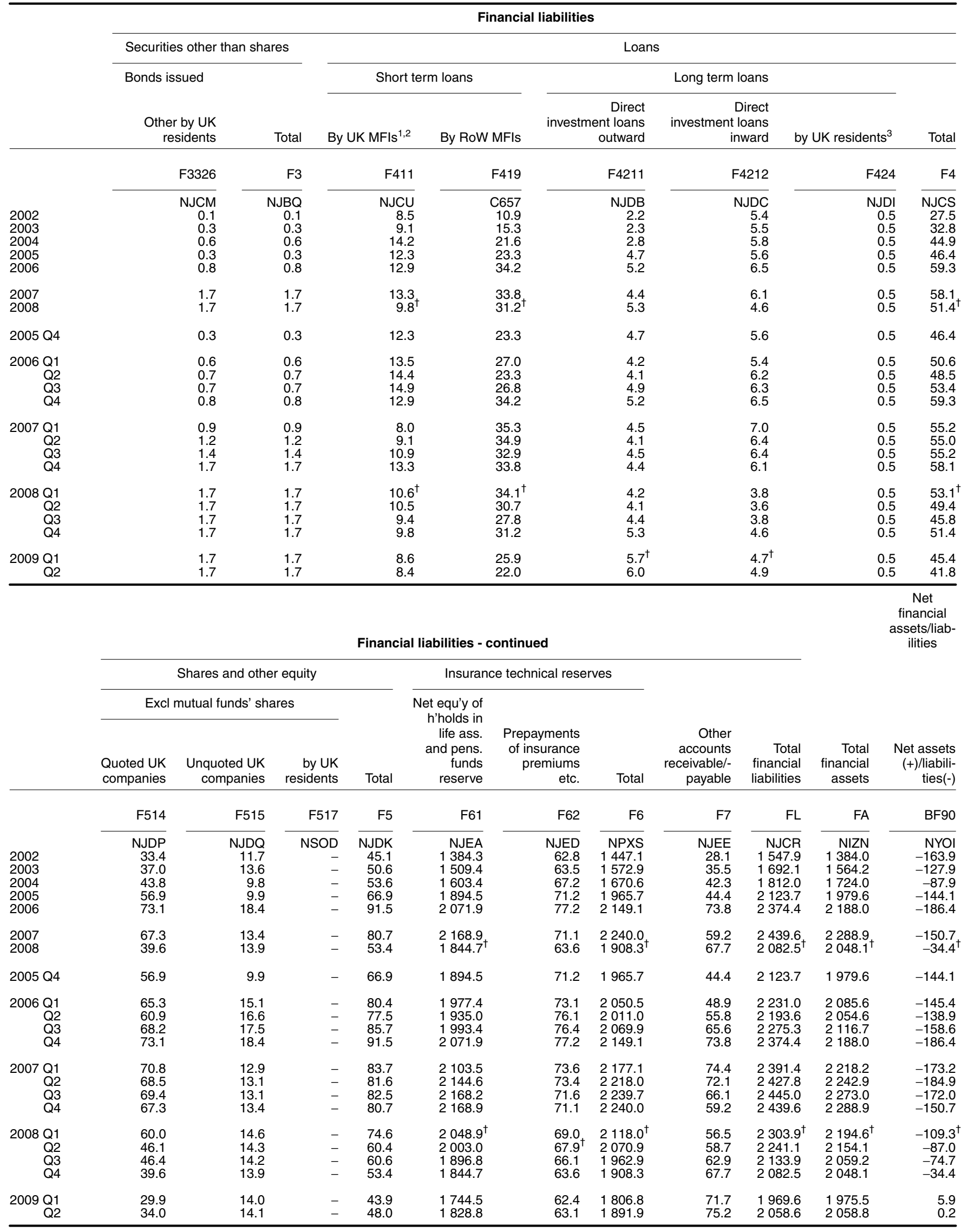

1 All loans secured on dwellings and all finance leasing are treated as long term loans

Sources: National Statistics;

2 Monetary financial institutions

3 Other than direct investment loans, loans secured on dwellings and loans for finance leasing 


\begin{tabular}{|c|c|c|c|c|c|c|c|c|c|c|c|c|c|c|c|c|}
\hline & \multicolumn{16}{|c|}{ Financial assets } \\
\hline & \multicolumn{5}{|c|}{ Currency and deposits } & \multicolumn{11}{|c|}{ Securities other than shares } \\
\hline & \multirow[b]{2}{*}{$\begin{array}{r}\text { Monetary } \\
\text { gold and } \\
\text { SDRs }\end{array}$} & \multirow{2}{*}{$\begin{array}{c}\text { Deposits } \\
\text { with UK } \\
\text { MFI'S }^{1}\end{array}$} & \multirow{2}{*}{$\begin{array}{r}\text { Deposits } \\
\text { with } \\
\text { rest of } \\
\text { the } \\
\text { world } \\
\text { MFl's }\end{array}$} & \multirow{2}{*}{$\begin{array}{r}\text { Deposits } \\
\text { other } \\
\text { than } \\
\text { with } \\
\text { MFI's }\end{array}$} & \multirow[b]{2}{*}{ Total } & \multicolumn{5}{|c|}{ Short term MMl's ${ }^{2}$ issued } & \multicolumn{4}{|c|}{ Bonds issued } & \multirow[b]{2}{*}{$\begin{array}{r}\text { Financi- } \\
\text { al } \\
\text { derivat- } \\
\text { ives }\end{array}$} & \multirow[b]{2}{*}{ Tota } \\
\hline & & & & & & $\begin{array}{r}\text { by UK } \\
\text { general } \\
\text { governm- } \\
\text { ent }\end{array}$ & $\begin{array}{l}\text { UK local } \\
\text { authori- } \\
\text { ty bills }\end{array}$ & $\begin{array}{c}\text { by UK } \\
\text { MFI's }\end{array}$ & $\begin{array}{r}\text { by other } \\
\text { UK } \\
\text { residen- } \\
\text { ts }\end{array}$ & $\begin{array}{l}\text { by the } \\
\text { rest of } \\
\text { the } \\
\text { world }\end{array}$ & $\begin{array}{r}\text { by UK } \\
\text { central } \\
\text { governm- } \\
\text { ent }\end{array}$ & $\begin{array}{r}\text { by UK } \\
\text { local } \\
\text { authori- } \\
\text { ties }\end{array}$ & $\begin{array}{r}\text { Other by } \\
\text { UK } \\
\text { residen- } \\
\text { ts }\end{array}$ & $\begin{array}{l}\text { by the } \\
\text { rest of } \\
\text { the } \\
\text { world }\end{array}$ & & \\
\hline & $\mathrm{F} 1$ & F221 & F229 & F29 & $\mathrm{F} 2$ & F3311 & F3312 & F3315 & F3316 & F3319 & F3321 & F3322 & F3326 & F3329 & F34 & F3 \\
\hline $\begin{array}{l}2002 \\
2003 \\
2004 \\
2005 \\
2006\end{array}$ & $\begin{array}{r}\text { NIFC } \\
2.4 \\
2.6 \\
2.5 \\
3.2 \\
3.4\end{array}$ & $\begin{array}{r}\text { NLVW } \\
20.3 \\
23.9 \\
26.9 \\
27.1 \\
34.6\end{array}$ & $\begin{array}{r}\text { NLWA } \\
5.9 \\
4.9 \\
3.3 \\
2.0 \\
1.2\end{array}$ & $\begin{array}{r}\text { NLWB } \\
1.0 \\
0.4 \\
0.4 \\
0.4 \\
0.2\end{array}$ & $\begin{array}{r}\text { NLUT } \\
27.1 \\
29.2 \\
30.6 \\
29.4 \\
35.9\end{array}$ & $\begin{array}{r}\text { NLWE } \\
0.2 \\
0.2 \\
0.1 \\
0.1 \\
0.1\end{array}$ & $\begin{array}{r}\text { NLWI } \\
- \\
- \\
- \\
- \\
-\end{array}$ & $\begin{array}{r}\text { NLWJ } \\
3.9 \\
3.9 \\
4.4 \\
4.5 \\
5.2\end{array}$ & $\begin{array}{r}\text { NLWO } \\
1.3 \\
0.2 \\
0.2 \\
0.3 \\
2.1\end{array}$ & $\begin{array}{r}\text { NLWP } \\
1.2 \\
0.2 \\
0.3 \\
1.7 \\
3.0\end{array}$ & $\begin{array}{r}\text { NLWR } \\
0.4 \\
0.3 \\
0.2 \\
0.2 \\
0.2\end{array}$ & $\begin{array}{r}\text { NLWU } \\
- \\
- \\
- \\
- \\
-\end{array}$ & $\begin{array}{r}\text { NLWY } \\
0.1 \\
0.1 \\
0.1 \\
1.1 \\
0.5\end{array}$ & $\begin{array}{r}\text { NLWZ } \\
16.8 \\
16.2 \\
17.1 \\
17.5 \\
15.3\end{array}$ & $\begin{array}{r}\text { ZYBQ } \\
0.2 \\
- \\
0.2 \\
0.6 \\
0.7\end{array}$ & $\begin{array}{r}\text { NLWC } \\
24.0 \\
20.9 \\
22.5 \\
26.0 \\
27.2\end{array}$ \\
\hline $\begin{array}{l}2007 \\
2008\end{array}$ & $\begin{array}{l}4.3 \\
6.3\end{array}$ & $\begin{array}{l}41.0 \\
57.4\end{array}$ & $\begin{array}{l}1.0 \\
4.6\end{array}$ & $\begin{array}{r}4.6 \\
17.7\end{array}$ & $\begin{array}{l}46.6 \\
79.7\end{array}$ & $\begin{array}{l}0.1 \\
0.1\end{array}$ & $\begin{array}{l}- \\
-\end{array}$ & $\begin{array}{l}2.3 \\
3.9\end{array}$ & $\begin{array}{l}1.3 \\
1.5\end{array}$ & $\begin{array}{l}5.5 \\
5.3\end{array}$ & $\begin{array}{l}0.1 \\
0.2\end{array}$ & $\begin{array}{l}- \\
-\end{array}$ & $\begin{array}{l}0.5 \\
5.5\end{array}$ & $\begin{array}{l}18.2 \\
24.4\end{array}$ & $\begin{array}{l}-0.4 \\
-3.5\end{array}$ & $\begin{array}{l}27.6 \\
37.4\end{array}$ \\
\hline 2005 Q4 & 3.2 & 27.1 & 2.0 & 0.4 & 29.4 & 0.1 & - & 4.5 & 0.3 & 1.7 & 0.2 & - & 1.1 & 17.5 & 0.6 & 26.0 \\
\hline $\begin{array}{r}2006 \text { Q1 } \\
\text { Q2 } \\
\text { Q3 } \\
\text { Q4 }\end{array}$ & $\begin{array}{l}3.5 \\
3.5 \\
3.4 \\
3.4\end{array}$ & $\begin{array}{l}30.2 \\
34.6 \\
38.0 \\
34.6\end{array}$ & $\begin{array}{l}1.8 \\
1.7 \\
1.6 \\
1.2\end{array}$ & $\begin{array}{l}1.9 \\
0.2 \\
0.2 \\
0.2\end{array}$ & $\begin{array}{l}34.0 \\
36.6 \\
39.8 \\
35.9\end{array}$ & $\begin{array}{l}0.3 \\
0.1 \\
0.1 \\
0.1\end{array}$ & $\begin{array}{l}- \\
- \\
- \\
-\end{array}$ & $\begin{array}{l}6.7 \\
6.8 \\
6.9 \\
5.2\end{array}$ & $\begin{array}{l}1.2 \\
0.9 \\
1.3 \\
2.1\end{array}$ & $\begin{array}{l}2.1 \\
1.6 \\
2.7 \\
3.0\end{array}$ & $\begin{array}{l}0.5 \\
0.3 \\
0.1 \\
0.2\end{array}$ & $\begin{array}{l}- \\
- \\
- \\
-\end{array}$ & $\begin{array}{l}1.1 \\
1.1 \\
1.1 \\
0.5\end{array}$ & $\begin{array}{l}17.2 \\
16.9 \\
15.9 \\
15.3\end{array}$ & $\begin{array}{l}0.3 \\
0.4 \\
0.6 \\
0.7\end{array}$ & $\begin{array}{l}29.3 \\
28.0 \\
28.7 \\
27.2\end{array}$ \\
\hline $\begin{array}{r}2007 \text { Q1 } \\
\text { Q2 } \\
\text { Q3 } \\
\text { Q4 }\end{array}$ & $\begin{array}{l}3.6 \\
3.4 \\
3.8 \\
4.3\end{array}$ & $\begin{array}{l}37.6 \\
41.0 \\
47.1 \\
41.0\end{array}$ & $\begin{array}{l}0.7 \\
0.8 \\
0.9 \\
1.0\end{array}$ & $\begin{array}{l}1.2 \\
0.4 \\
0.8 \\
4.6\end{array}$ & $\begin{array}{l}39.6 \\
42.2 \\
48.8 \\
46.6\end{array}$ & $\begin{array}{l}0.1 \\
0.1 \\
0.1 \\
0.1\end{array}$ & $\begin{array}{l}- \\
- \\
- \\
-\end{array}$ & $\begin{array}{l}5.7 \\
7.4 \\
3.8 \\
2.3\end{array}$ & $\begin{array}{l}2.0 \\
1.3 \\
1.4 \\
1.3\end{array}$ & $\begin{array}{l}2.8 \\
4.0 \\
5.5 \\
5.5\end{array}$ & $\begin{array}{l}0.1 \\
0.2 \\
0.2 \\
0.1\end{array}$ & $\begin{array}{l}- \\
- \\
- \\
-\end{array}$ & $\begin{array}{l}0.5 \\
0.5 \\
0.5 \\
0.5\end{array}$ & $\begin{array}{l}15.4 \\
14.0 \\
16.1 \\
18.2\end{array}$ & $\begin{array}{r}0.6 \\
0.7 \\
0.2 \\
-0.4\end{array}$ & $\begin{array}{l}27.2 \\
28.2 \\
27.7 \\
27.6\end{array}$ \\
\hline $\begin{array}{r}2008 \text { Q1 } \\
\text { Q2 } \\
\text { Q3 } \\
\text { Q4 }\end{array}$ & $\begin{array}{l}4.9 \\
4.9 \\
5.2 \\
6.3\end{array}$ & $\begin{array}{l}36.5 \\
40.4 \\
60.5 \\
57.4\end{array}$ & $\begin{array}{l}1.9 \\
2.4 \\
1.8 \\
4.6\end{array}$ & $\begin{array}{r}6.6 \\
4.6 \\
7.6 \\
17.7\end{array}$ & $\begin{array}{l}45.1 \\
47.4 \\
69.9 \\
79.7\end{array}$ & $\begin{array}{l}0.1 \\
\overline{-} \\
0.1 \\
0.1\end{array}$ & $\begin{array}{l}- \\
- \\
- \\
-\end{array}$ & $\begin{array}{l}1.7 \\
1.7 \\
1.3 \\
3.9\end{array}$ & $\begin{array}{l}1.5 \\
1.5 \\
1.9 \\
1.5\end{array}$ & $\begin{array}{l}8.7 \\
1.4 \\
7.1 \\
5.3\end{array}$ & $\begin{array}{l}0.1 \\
0.2 \\
0.2 \\
0.2\end{array}$ & $\begin{array}{l}- \\
- \\
- \\
-\end{array}$ & $\begin{array}{l}0.5 \\
0.5 \\
0.5 \\
5.5\end{array}$ & $\begin{array}{l}19.9 \\
18.8 \\
18.9 \\
24.4\end{array}$ & $\begin{array}{l}-1.3 \\
-0.9 \\
-1.1 \\
-3.5\end{array}$ & $\begin{array}{l}31.2 \\
23.2 \\
28.8 \\
37.4\end{array}$ \\
\hline $\begin{array}{r}2009 \text { Q1 } \\
\text { Q2 }\end{array}$ & $\begin{array}{l}6.7 \\
5.9\end{array}$ & $\begin{array}{l}50.8 \\
56.1\end{array}$ & $\begin{array}{l}4.3 \\
4.9\end{array}$ & $\begin{array}{l}34.8 \\
26.9\end{array}$ & $\begin{array}{l}89.9 \\
87.9\end{array}$ & $\begin{array}{l}0.1 \\
0.1\end{array}$ & $\begin{array}{l}- \\
-\end{array}$ & $\begin{array}{l}3.6 \\
3.7\end{array}$ & $\begin{array}{l}1.1 \\
1.9\end{array}$ & $\begin{array}{l}5.8 \\
9.8\end{array}$ & $\begin{array}{l}0.2 \\
0.2\end{array}$ & - & $\begin{array}{l}5.5 \\
5.7\end{array}$ & $\begin{array}{l}23.7 \\
21.3\end{array}$ & $\begin{array}{l}-2.8 \\
-1.5\end{array}$ & $\begin{array}{l}37.1 \\
41.2\end{array}$ \\
\hline
\end{tabular}

\begin{tabular}{|c|c|c|c|c|c|c|c|c|c|c|c|}
\hline & \multicolumn{11}{|c|}{ Financial assets - continued } \\
\hline & \multicolumn{3}{|c|}{ Loans } & \multicolumn{5}{|c|}{ Shares and other equity } & \multirow{3}{*}{$\begin{array}{r}\text { Insurance } \\
\text { technical } \\
\text { reserves } \\
\text { Prepayments } \\
\text { of insurance } \\
\text { premiums } \\
\text { etc. }\end{array}$} & \multirow{3}{*}{$\begin{array}{r}\text { Other } \\
\text { accounts } \\
\text { receivable/- } \\
\text { payable }\end{array}$} & \multirow{3}{*}{$\begin{array}{r}\text { Total } \\
\text { financial } \\
\text { assets } \\
\end{array}$} \\
\hline & \multicolumn{2}{|c|}{ Long term loans } & \multirow[b]{2}{*}{ Total } & \multicolumn{4}{|c|}{ Excl mutual funds' shares } & \multirow[b]{2}{*}{ Total } & & & \\
\hline & $\begin{array}{l}\text { Secured on } \\
\text { dwellings }\end{array}$ & $\begin{array}{l}\text { by UK } \\
\text { residents }{ }^{3}\end{array}$ & & $\begin{array}{r}\text { Quoted UK } \\
\text { shares }\end{array}$ & $\begin{array}{r}\text { Unquoted UK } \\
\text { shares }\end{array}$ & $\begin{array}{r}\text { Other UK } \\
\text { equity }\end{array}$ & $\begin{array}{l}\text { by the rest } \\
\text { of the world }\end{array}$ & & & & \\
\hline & $\mathrm{F} 422$ & $\mathrm{~F} 424$ & $\mathrm{~F} 4$ & F514 & F515 & F516 & F519 & F5 & F62 & F7 & FA \\
\hline $\begin{array}{l}2002 \\
2003 \\
2004 \\
2005 \\
2006\end{array}$ & $\begin{array}{r}\text { NLXP } \\
0.9 \\
1.1 \\
1.1 \\
1.4 \\
1.7\end{array}$ & $\begin{array}{r}\text { NLXU } \\
68.6 \\
66.9 \\
69.6 \\
74.5 \\
78.0\end{array}$ & $\begin{array}{r}\text { NLXE } \\
69.6 \\
68.0 \\
70.7 \\
75.9 \\
79.6\end{array}$ & $\begin{array}{r}\text { NLYB } \\
1.0 \\
1.2 \\
0.8 \\
1.1 \\
1.6\end{array}$ & $\begin{array}{r}\text { NLYC } \\
1.3 \\
1.3 \\
2.1 \\
2.1 \\
4.7\end{array}$ & $\begin{array}{r}\mathrm{H} 4 \mathrm{O} 9 \\
95.8 \\
104.4 \\
113.3 \\
129.5 \\
120.7\end{array}$ & $\begin{array}{r}\text { NLYG } \\
7.5 \\
7.7 \\
8.0 \\
8.7 \\
9.5\end{array}$ & $\begin{array}{r}\text { NLXW } \\
105.6 \\
114.7 \\
124.2 \\
141.4 \\
136.5\end{array}$ & $\begin{array}{r}\text { NLYP } \\
0.9 \\
0.8 \\
0.8 \\
0.8 \\
0.8\end{array}$ & $\begin{array}{r}\text { NLYQ } \\
41.5 \\
45.4 \\
47.5 \\
53.4 \\
54.3\end{array}$ & $\begin{array}{r}\text { NPUP } \\
271.2 \\
281.5 \\
298.8 \\
330.1 \\
337.8\end{array}$ \\
\hline $\begin{array}{l}2007 \\
2008\end{array}$ & $\begin{array}{l}2.2^{\dagger} \\
3.0^{\dagger}\end{array}$ & $\begin{array}{l}83.6 \\
89.4\end{array}$ & $\begin{array}{l}85.8 \\
92.4^{\dagger}\end{array}$ & $\begin{array}{r}1.4 \\
13.1\end{array}$ & $\begin{array}{l}3.0 \\
6.7\end{array}$ & $\begin{array}{l}119.1^{\dagger} \\
122.4^{\dagger}\end{array}$ & $\begin{array}{l}10.2 \\
11.2\end{array}$ & $\begin{array}{l}133.8^{\dagger} \\
153.3^{\dagger}\end{array}$ & $\begin{array}{l}0.9 \\
0.8\end{array}$ & $\begin{array}{l}53.0^{\dagger} \\
69.9^{\dagger}\end{array}$ & $\begin{array}{l}352.1^{\dagger} \\
439.8^{\dagger}\end{array}$ \\
\hline 2005 Q4 & 1.4 & 74.5 & 75.9 & 1.1 & 2.1 & 129.5 & 8.7 & 141.4 & 0.8 & 53.4 & 330.1 \\
\hline $\begin{array}{r}2006 \text { Q1 } \\
\text { Q2 } \\
\text { Q3 } \\
\text { Q4 }\end{array}$ & $\begin{array}{l}1.5 \\
1.5 \\
1.6 \\
1.7\end{array}$ & $\begin{array}{l}75.4 \\
75.9 \\
77.4 \\
78.0\end{array}$ & $\begin{array}{l}76.8 \\
77.4 \\
79.0 \\
79.6\end{array}$ & $\begin{array}{l}1.6 \\
1.6 \\
1.6 \\
1.6\end{array}$ & $\begin{array}{l}3.1 \\
3.6 \\
4.1 \\
4.7\end{array}$ & $\begin{array}{l}129.0 \\
129.8 \\
124.4 \\
120.7\end{array}$ & $\begin{array}{l}8.7 \\
9.2 \\
9.4 \\
9.5\end{array}$ & $\begin{array}{l}142.3 \\
144.2 \\
139.6 \\
136.5\end{array}$ & $\begin{array}{l}0.8 \\
0.8 \\
0.8 \\
0.8\end{array}$ & $\begin{array}{l}57.3 \\
53.2 \\
53.3 \\
54.3\end{array}$ & $\begin{array}{l}344.1 \\
343.7 \\
344.6 \\
337.8\end{array}$ \\
\hline $\begin{array}{r}2007 \text { Q1 } \\
\text { Q2 } \\
\text { Q3 } \\
\text { Q4 }\end{array}$ & $\begin{array}{l}1.8 \\
1.8 \\
2.0 \\
2.2\end{array}$ & $\begin{array}{l}78.3 \\
78.3 \\
81.9 \\
83.6\end{array}$ & $\begin{array}{l}80.1 \\
80.1 \\
84.0 \\
85.8\end{array}$ & $\begin{array}{l}1.6 \\
1.5 \\
1.5 \\
1.4\end{array}$ & $\begin{array}{l}3.4 \\
3.3 \\
3.0 \\
3.0\end{array}$ & $\begin{array}{l}116.3 \\
116.7 \\
118.7 \\
119.1\end{array}$ & $\begin{array}{r}9.6 \\
10.2 \\
10.2 \\
10.2\end{array}$ & $\begin{array}{l}130.8 \\
131.8 \\
133.4 \\
133.8\end{array}$ & $\begin{array}{l}0.8 \\
0.8 \\
0.8 \\
0.9\end{array}$ & $\begin{array}{l}62.5 \\
56.8 \\
54.3 \\
53.0\end{array}$ & $\begin{array}{l}344.6 \\
343.3 \\
352.8 \\
352.1\end{array}$ \\
\hline $\begin{array}{r}2008 \text { Q1 } \\
\text { Q2 } \\
\text { Q3 } \\
\text { Q4 }\end{array}$ & $\begin{array}{l}2.7 \\
2.8 \\
2.9^{\dagger} \\
3.0^{\dagger}\end{array}$ & $\begin{array}{l}85.3 \\
86.4 \\
87.8 \\
89.4\end{array}$ & $\begin{array}{l}88.0 \\
89.2 \\
90.7 \\
92.4^{\dagger}\end{array}$ & $\begin{array}{r}1.3 \\
1.3 \\
1.0 \\
13.1\end{array}$ & $\begin{array}{l}3.0 \\
3.1 \\
6.6 \\
6.7\end{array}$ & $\begin{array}{l}119.4 \\
121.3^{\dagger} \\
121.9 \\
122.4\end{array}$ & $\begin{array}{l}10.3 \\
10.8 \\
11.0 \\
11.2\end{array}$ & $\begin{array}{l}134.0^{\dagger} \\
136.4^{\dagger} \\
140.4 \\
153.3\end{array}$ & $\begin{array}{l}0.8 \\
0.8 \\
0.8 \\
0.8\end{array}$ & $\begin{array}{l}61.4^{\dagger} \\
57.7 \\
66.7 \\
69.9\end{array}$ & $\begin{array}{l}365.3^{\dagger} \\
359.6 \\
402.6 \\
439.8\end{array}$ \\
\hline $\begin{array}{r}2009 \text { Q1 } \\
\text { Q2 }\end{array}$ & $\begin{array}{l}3.0 \\
2.8\end{array}$ & $\begin{array}{l}89.5 \\
89.9\end{array}$ & $\begin{array}{l}92.5 \\
92.7\end{array}$ & $\begin{array}{l}12.8 \\
13.7\end{array}$ & $\begin{array}{l}6.7 \\
6.6\end{array}$ & $\begin{array}{l}122.6 \\
123.4\end{array}$ & $\begin{array}{l}11.2 \\
11.3\end{array}$ & $\begin{array}{l}153.3 \\
155.0\end{array}$ & $\begin{array}{l}0.7 \\
0.8\end{array}$ & $\begin{array}{l}73.6 \\
69.5\end{array}$ & $\begin{array}{l}453.7 \\
452.9\end{array}$ \\
\hline $\begin{array}{l}1 \text { Monet } \\
2 \text { Money } \\
3 \text { Other } \\
\text { used fo }\end{array}$ & $\begin{array}{l}\text { ancial institut } \\
\text { et instrument } \\
\text { lirect investm } \\
\text { nce leasing }\end{array}$ & $\begin{array}{l}\text { ons } \\
\text { ent loans, I }\end{array}$ & & & 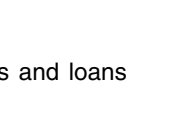 & & & & & $\begin{array}{r}\text { ces: National } \\
\text { Bank o }\end{array}$ & $\begin{array}{l}\text { Statistics; } \\
\text { f England }\end{array}$ \\
\hline
\end{tabular}




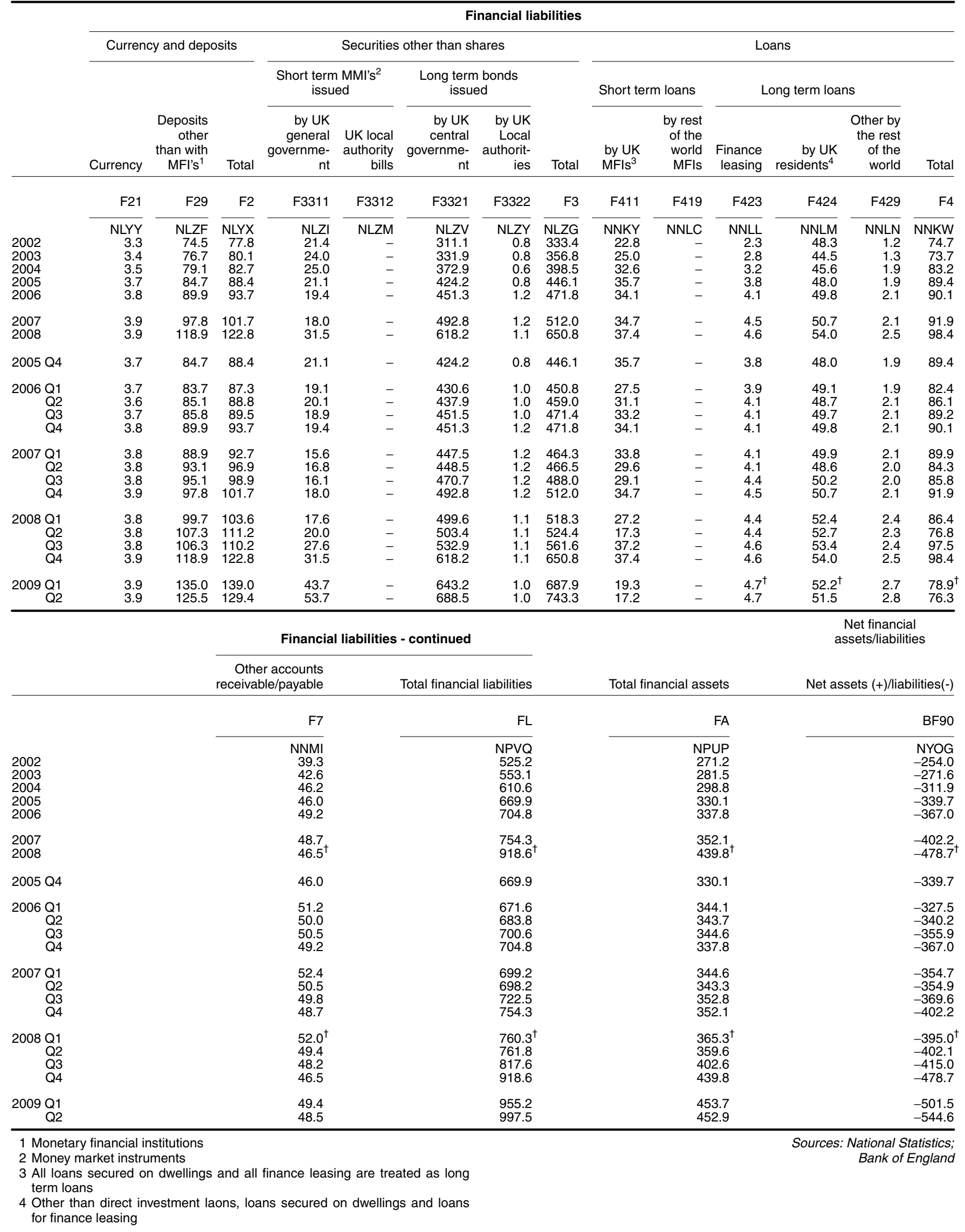




\begin{tabular}{|c|c|c|c|c|c|c|c|c|c|c|c|c|c|}
\hline & \multicolumn{13}{|c|}{ Financial assets } \\
\hline & \multirow[b]{3}{*}{$\begin{array}{r}\text { Monetary } \\
\text { gold and } \\
\text { SDRs }\end{array}$} & \multicolumn{4}{|c|}{ Currency and deposits } & \multicolumn{8}{|c|}{ Securities other than shares } \\
\hline & & \multirow[b]{2}{*}{$\begin{array}{c}\text { Deposits } \\
\text { with UK } \\
\text { MFI's }^{1}\end{array}$} & \multirow[b]{2}{*}{$\begin{array}{r}\text { Deposits } \\
\text { with rest } \\
\text { of the } \\
\text { world } \\
\text { MFl's }\end{array}$} & \multirow[b]{2}{*}{$\begin{array}{l}\text { Deposits } \\
\text { other than } \\
\text { with MFI's }\end{array}$} & \multirow[b]{2}{*}{ Total } & \multicolumn{3}{|c|}{ Short term MMl's ${ }^{2}$ issued } & \multicolumn{4}{|c|}{ Bonds issued } & \multirow[b]{2}{*}{ Tota } \\
\hline & & & & & & by UK MFIs & $\begin{array}{r}\text { by other } \\
\text { UK } \\
\text { residents }\end{array}$ & $\begin{array}{l}\text { by the } \\
\text { rest of } \\
\text { the world }\end{array}$ & $\begin{array}{r}\text { by UK } \\
\text { local } \\
\text { authoriti- } \\
\text { es }\end{array}$ & $\begin{array}{r}\text { Other by } \\
\text { UK } \\
\text { residents }\end{array}$ & $\begin{array}{l}\text { by the } \\
\text { rest of } \\
\text { the world }\end{array}$ & $\begin{array}{r}\text { Financial } \\
\text { derivativ- } \\
\text { es }\end{array}$ & \\
\hline & $\mathrm{F} 1$ & $\mathrm{~F} 221$ & F229 & F29 & $\mathrm{F} 2$ & F3315 & F3316 & F3319 & F3322 & F3326 & F3329 & F34 & F3 \\
\hline $\begin{array}{l}2002 \\
2003 \\
2004 \\
2005 \\
2006\end{array}$ & $\begin{array}{r}\text { NIFC } \\
2.4 \\
2.6 \\
2.5 \\
3.2 \\
3.4\end{array}$ & $\begin{array}{r}\mathrm{NIFI} \\
4.6 \\
6.8 \\
5.5 \\
5.6 \\
7.7\end{array}$ & $\begin{array}{r}\text { NIFM } \\
5.9 \\
4.9 \\
3.3 \\
2.0 \\
1.2\end{array}$ & $\begin{array}{r}\text { NIFN } \\
0.7 \\
- \\
- \\
- \\
-\end{array}$ & $\begin{array}{r}\text { NIFF } \\
11.2 \\
11.7 \\
8.8 \\
7.6 \\
8.9\end{array}$ & $\begin{array}{r}\text { NSUO } \\
0.1 \\
- \\
0.8 \\
1.0 \\
2.7\end{array}$ & $\begin{array}{r}\text { NSRH } \\
1.1 \\
- \\
- \\
\overline{-} \\
1 . \overline{2}\end{array}$ & $\begin{array}{r}\text { NIGB } \\
1.2 \\
0.2 \\
0.3 \\
1.7 \\
3.0\end{array}$ & $\begin{array}{r}\text { NIGG } \\
- \\
- \\
- \\
- \\
-\end{array}$ & $\begin{array}{r}\text { NIGK } \\
0.1 \\
0.1 \\
0.1 \\
0.9 \\
0.3\end{array}$ & $\begin{array}{r}\text { NIGL } \\
16.8 \\
16.2 \\
17.1 \\
17.5 \\
15.3\end{array}$ & $\begin{array}{r}\text { ZYBQ } \\
0.2 \\
- \\
0.2 \\
0.6 \\
0.7\end{array}$ & $\begin{array}{r}\text { NIFO } \\
19.4 \\
16.4 \\
18.4 \\
21.8 \\
23.3\end{array}$ \\
\hline $\begin{array}{l}2007 \\
2008\end{array}$ & $\begin{array}{l}4.3 \\
6.3\end{array}$ & $\begin{array}{r}9.8 \\
28.5\end{array}$ & $\begin{array}{l}1.0 \\
4.6\end{array}$ & $\begin{array}{r}3.8 \\
14.8\end{array}$ & $\begin{array}{l}14.6 \\
47.9\end{array}$ & $\begin{array}{l}0.8 \\
2.6\end{array}$ & $\begin{array}{l}0.1 \\
0.1\end{array}$ & $\begin{array}{l}5.5 \\
5.3\end{array}$ & $\begin{array}{l}- \\
-\end{array}$ & $\begin{array}{l}0.3 \\
5.3\end{array}$ & $\begin{array}{l}18.2 \\
24.4\end{array}$ & $\begin{array}{l}-0.4 \\
-3.5\end{array}$ & $\begin{array}{l}24.4 \\
34.1\end{array}$ \\
\hline 2005 Q4 & 3.2 & 5.6 & 2.0 & - & 7.6 & 1.0 & - & 1.7 & - & 0.9 & 17.5 & 0.6 & 21.8 \\
\hline $\begin{array}{r}2006 \text { Q1 } \\
\text { Q2 } \\
\text { Q3 } \\
\text { Q4 }\end{array}$ & $\begin{array}{l}3.5 \\
3.5 \\
3.4 \\
3.4\end{array}$ & $\begin{array}{r}9.2 \\
9.3 \\
10.8 \\
7.7\end{array}$ & $\begin{array}{l}1.8 \\
1.7 \\
1.6 \\
1.2\end{array}$ & $\begin{array}{c}1.6 \\
- \\
- \\
-\end{array}$ & $\begin{array}{r}12.6 \\
11.0 \\
12.5 \\
8.9\end{array}$ & $\begin{array}{l}3.8 \\
3.8 \\
4.2 \\
2.7\end{array}$ & $\begin{array}{l}0.8 \\
0.3 \\
0.6 \\
1.2\end{array}$ & $\begin{array}{l}2.1 \\
1.6 \\
2.7 \\
3.0\end{array}$ & $\begin{array}{l}- \\
- \\
- \\
-\end{array}$ & $\begin{array}{l}0.9 \\
0.9 \\
0.9 \\
0.3\end{array}$ & $\begin{array}{l}17.2 \\
16.9 \\
15.9 \\
15.3\end{array}$ & $\begin{array}{l}0.3 \\
0.4 \\
0.6 \\
0.7\end{array}$ & $\begin{array}{l}25.1 \\
23.8 \\
24.9 \\
23.3\end{array}$ \\
\hline $\begin{array}{r}2007 \text { Q1 } \\
\text { Q2 } \\
\text { Q3 } \\
\text { Q4 }\end{array}$ & $\begin{array}{l}3.6 \\
3.4 \\
3.8 \\
4.3\end{array}$ & $\begin{array}{r}13.7 \\
12.8 \\
16.0 \\
9.8\end{array}$ & $\begin{array}{l}0.7 \\
0.8 \\
0.9 \\
1.0\end{array}$ & $\begin{array}{l}1.0 \\
0.1 \\
0.3 \\
3.8\end{array}$ & $\begin{array}{l}15.4 \\
13.7 \\
17.2 \\
14.6\end{array}$ & $\begin{array}{l}3.7 \\
5.7 \\
2.3 \\
0.8\end{array}$ & $\begin{array}{l}1.2 \\
0.3 \\
0.1 \\
0.1\end{array}$ & $\begin{array}{l}2.8 \\
4.0 \\
5.5 \\
5.5\end{array}$ & $\begin{array}{l}- \\
- \\
- \\
-\end{array}$ & $\begin{array}{l}0.3 \\
0.3 \\
0.3 \\
0.3\end{array}$ & $\begin{array}{l}15.4 \\
14.0 \\
16.1 \\
18.2\end{array}$ & $\begin{array}{r}0.6 \\
0.7 \\
0.2 \\
-0.4\end{array}$ & $\begin{array}{l}24.0 \\
25.0 \\
24.4 \\
24.4\end{array}$ \\
\hline $\begin{array}{r}2008 \text { Q1 } \\
\text { Q2 } \\
\text { Q3 } \\
\text { Q4 }\end{array}$ & $\begin{array}{l}4.9 \\
4.9 \\
5.2 \\
6.3\end{array}$ & $\begin{array}{r}8.1 \\
8.3 \\
28.1 \\
28.5\end{array}$ & $\begin{array}{l}1.9 \\
2.4 \\
1.8 \\
4.6\end{array}$ & $\begin{array}{r}6.0 \\
3.7 \\
6.2 \\
14.8\end{array}$ & $\begin{array}{l}16.0 \\
14.4 \\
36.2 \\
47.9\end{array}$ & $\begin{array}{r}0.3 \\
0.2 \\
-\overline{6}\end{array}$ & $\begin{array}{l}0.1 \\
0.1 \\
0.1 \\
0.1\end{array}$ & $\begin{array}{l}8.7 \\
1.4 \\
7.1 \\
5.3\end{array}$ & $\begin{array}{l}- \\
- \\
- \\
-\end{array}$ & $\begin{array}{l}0.3 \\
0.3 \\
0.3 \\
5.3\end{array}$ & $\begin{array}{l}19.9 \\
18.8 \\
18.9 \\
24.4\end{array}$ & $\begin{array}{l}-1.3 \\
-0.9 \\
-1.1 \\
-3.5\end{array}$ & $\begin{array}{l}27.9 \\
19.8 \\
25.3 \\
34.1\end{array}$ \\
\hline $\begin{array}{r}2009 \text { Q1 } \\
\text { Q2 }\end{array}$ & $\begin{array}{l}6.7 \\
5.9\end{array}$ & $\begin{array}{l}28.8 \\
32.9\end{array}$ & $\begin{array}{l}4.3 \\
4.9\end{array}$ & $\begin{array}{l}30.6 \\
20.2\end{array}$ & $\begin{array}{l}63.7 \\
58.0\end{array}$ & $\begin{array}{l}2.3 \\
2.5\end{array}$ & $\begin{array}{l}0.2 \\
1.2\end{array}$ & $\begin{array}{l}5.8 \\
9.8\end{array}$ & $\begin{array}{l}- \\
-\end{array}$ & $\begin{array}{l}5.3 \\
5.5\end{array}$ & $\begin{array}{l}23.7 \\
21.3\end{array}$ & $\begin{array}{l}-2.8 \\
-1.5\end{array}$ & $\begin{array}{l}34.5 \\
38.8\end{array}$ \\
\hline
\end{tabular}

\begin{tabular}{|c|c|c|c|c|c|c|c|c|c|c|c|}
\hline & \multicolumn{11}{|c|}{ Financial assets - continued } \\
\hline & \multicolumn{3}{|c|}{ Loans } & \multicolumn{6}{|c|}{ Shares and other equity } & \multirow{3}{*}{$\begin{array}{r}\text { Other } \\
\text { accounts } \\
\text { receivable/- } \\
\text { payable }\end{array}$} & \multirow{3}{*}{$\begin{array}{r}\text { Total } \\
\text { financial } \\
\text { assets }\end{array}$} \\
\hline & \multicolumn{2}{|c|}{ Long term loans } & \multirow[b]{2}{*}{ Total } & \multicolumn{5}{|c|}{ Excl mutual funds' shares } & \multirow[b]{2}{*}{ Total } & & \\
\hline & $\begin{array}{l}\text { Secured on } \\
\text { dwellings }\end{array}$ & $\begin{array}{l}\text { by UK } \\
\text { residents }^{3}\end{array}$ & & $\begin{array}{r}\text { Quoted UK } \\
\text { shares }\end{array}$ & $\begin{array}{r}\text { Other UK } \\
\text { equity }\end{array}$ & $\begin{array}{r}\text { Unquoted UK } \\
\text { shares }\end{array}$ & $\begin{array}{r}\text { by UK } \\
\text { residents }\end{array}$ & $\begin{array}{l}\text { by the rest } \\
\text { of the world }\end{array}$ & & & \\
\hline & $\mathrm{F} 422$ & $\mathrm{~F} 424$ & $\mathrm{~F} 4$ & F514 & F515 & F516 & F517 & F519 & F5 & F7 & FA \\
\hline $\begin{array}{l}2002 \\
2003 \\
2004 \\
2005 \\
2006\end{array}$ & $\begin{array}{r}\text { NIHB } \\
0.1 \\
0.1 \\
0.1 \\
0.1 \\
0.1\end{array}$ & $\begin{array}{r}\text { NIHG } \\
68.4 \\
66.6 \\
69.4 \\
74.3 \\
77.7\end{array}$ & $\begin{array}{r}\text { NIGQ } \\
68.4 \\
66.7 \\
69.4 \\
74.4 \\
77.8\end{array}$ & $\begin{array}{r}\text { NIHN } \\
- \\
- \\
\overline{-} \\
0.3 \\
0.6\end{array}$ & $\begin{array}{r}\mathrm{H} 4 \mathrm{O} 7 \\
9.8 \\
9.3 \\
7.1 \\
12.7 \\
9.8\end{array}$ & $\begin{array}{r}\mathrm{NIHO} \\
0.9 \\
0.9 \\
1.4 \\
1.4 \\
3.9\end{array}$ & $\begin{array}{r}\text { NSNX } \\
- \\
- \\
- \\
- \\
-\end{array}$ & $\begin{array}{r}\text { NIHS } \\
7.5 \\
7.7 \\
8.0 \\
8.7 \\
9.5\end{array}$ & $\begin{array}{c}\text { NIHI } \\
18.2 \\
18.0 \\
16.5 \\
23.1 \\
23.8\end{array}$ & $\begin{array}{l}\text { NIIC } \\
41.2 \\
44.5 \\
47.2 \\
52.5 \\
55.2\end{array}$ & $\begin{array}{c}\text { NIGP } \\
160.8 \\
159.9 \\
162.8 \\
182.4 \\
192.4\end{array}$ \\
\hline $\begin{array}{l}2007 \\
2008\end{array}$ & $\begin{array}{l}0.1 \\
0.1\end{array}$ & $\begin{array}{l}83.3 \\
89.2\end{array}$ & $\begin{array}{l}83.4 \\
89.2\end{array}$ & $\begin{array}{r}0.5 \\
12.8\end{array}$ & $\begin{array}{l}7.8 \\
8.9\end{array}$ & $\begin{array}{l}2.3 \\
5.9\end{array}$ & $\begin{array}{l}- \\
-\end{array}$ & $\begin{array}{l}10.2 \\
11.2\end{array}$ & $\begin{array}{l}20.8 \\
38.8\end{array}$ & $\begin{array}{l}54.0^{\dagger} \\
68.7^{\dagger}\end{array}$ & $\begin{array}{l}201.7^{\dagger} \\
285.1^{\dagger}\end{array}$ \\
\hline 2005 Q4 & 0.1 & 74.3 & 74.4 & 0.3 & 12.7 & 1.4 & - & 8.7 & 23.1 & 52.5 & 182.4 \\
\hline $\begin{array}{r}2006 \text { Q1 } \\
\text { Q2 } \\
\text { Q3 } \\
\text { Q4 }\end{array}$ & $\begin{array}{l}0.1 \\
0.1 \\
0.1 \\
0.1\end{array}$ & $\begin{array}{l}75.1 \\
75.7 \\
77.2 \\
77.7\end{array}$ & $\begin{array}{l}75.2 \\
75.8 \\
77.2 \\
77.8\end{array}$ & $\begin{array}{l}0.6 \\
0.5 \\
0.5 \\
0.6\end{array}$ & $\begin{array}{r}10.9 \\
12.4 \\
10.2 \\
9.8\end{array}$ & $\begin{array}{l}2.3 \\
2.8 \\
3.4 \\
3.9\end{array}$ & $\begin{array}{l}- \\
- \\
- \\
-\end{array}$ & $\begin{array}{l}8.7 \\
9.2 \\
9.4 \\
9.5\end{array}$ & $\begin{array}{l}22.5 \\
24.9 \\
23.6 \\
23.8\end{array}$ & $\begin{array}{l}53.5 \\
51.6 \\
53.0 \\
55.2\end{array}$ & $\begin{array}{l}192.4 \\
190.6 \\
194.6 \\
192.4\end{array}$ \\
\hline $\begin{array}{r}2007 \text { Q1 } \\
\text { Q2 } \\
\text { Q3 } \\
\text { Q4 }\end{array}$ & $\begin{array}{l}0.1 \\
0.1 \\
0.1 \\
0.1\end{array}$ & $\begin{array}{l}78.1 \\
78.0 \\
81.7 \\
83.3\end{array}$ & $\begin{array}{l}78.2 \\
78.1 \\
81.7 \\
83.4\end{array}$ & $\begin{array}{l}0.6 \\
0.6 \\
0.5 \\
0.5\end{array}$ & $\begin{array}{l}9.0 \\
6.6 \\
7.9 \\
7.8\end{array}$ & $\begin{array}{l}2.6 \\
2.6 \\
2.3 \\
2.3\end{array}$ & $\begin{array}{l}- \\
- \\
- \\
-\end{array}$ & $\begin{array}{r}9.6 \\
10.2 \\
10.2 \\
10.2\end{array}$ & $\begin{array}{l}21.8 \\
20.0 \\
21.0 \\
20.8\end{array}$ & $\begin{array}{l}58.5 \\
55.2 \\
54.1 \\
54.0\end{array}$ & $\begin{array}{l}201.5 \\
195.5 \\
202.3 \\
201.7\end{array}$ \\
\hline $\begin{array}{r}2008 \text { Q1 } \\
\text { Q2 } \\
\text { Q3 } \\
\text { Q4 }\end{array}$ & $\begin{array}{l}0.1 \\
0.1 \\
0.1 \\
0.1\end{array}$ & $\begin{array}{l}85.0 \\
86.2 \\
87.6 \\
89.2\end{array}$ & $\begin{array}{l}85.1 \\
86.3 \\
87.7 \\
89.2\end{array}$ & $\begin{array}{r}0.5 \\
0.6 \\
0.3 \\
12.8\end{array}$ & $\begin{array}{l}7.6 \\
7.6 \\
8.3 \\
8.9\end{array}$ & $\begin{array}{l}2.3 \\
2.4 \\
5.8 \\
5.9\end{array}$ & $\begin{array}{l}- \\
- \\
- \\
-\end{array}$ & $\begin{array}{l}10.3 \\
10.8 \\
11.0 \\
11.2\end{array}$ & $\begin{array}{l}20.7 \\
21.3 \\
25.4 \\
38.8\end{array}$ & $\begin{array}{l}55.0^{\dagger} \\
53.9 \\
64.4 \\
68.7\end{array}$ & $\begin{array}{l}209.6^{\dagger} \\
200.6 \\
244.2 \\
285.1\end{array}$ \\
\hline $\begin{aligned} 2009 \text { Q1 } \\
\text { Q2 }\end{aligned}$ & $\begin{array}{l}0.1 \\
0.1\end{array}$ & $\begin{array}{l}89.2 \\
89.7\end{array}$ & $\begin{array}{l}89.3 \\
89.8\end{array}$ & $\begin{array}{l}12.8 \\
13.4\end{array}$ & $\begin{array}{l}9.3 \\
9.5\end{array}$ & $\begin{array}{l}6.0 \\
5.9\end{array}$ & $\begin{array}{l}- \\
-\end{array}$ & $\begin{array}{l}11.2 \\
11.3\end{array}$ & $\begin{array}{l}39.3 \\
40.1\end{array}$ & $\begin{array}{l}66.8 \\
65.2\end{array}$ & $\begin{array}{l}300.2 \\
297.9\end{array}$ \\
\hline
\end{tabular}




\begin{tabular}{|c|c|c|c|c|c|c|c|c|c|c|c|c|c|c|}
\hline & \multicolumn{2}{|c|}{$\begin{array}{l}\text { Currency and } \\
\text { deposits }\end{array}$} & \multicolumn{6}{|c|}{ Securities other than shares } & \multicolumn{6}{|c|}{ Loans } \\
\hline & \multirow[b]{2}{*}{ Currency } & \multirow[b]{2}{*}{$\begin{array}{l}\text { Deposits } \\
\text { other } \\
\text { than with } \\
\text { MFl's }^{1}\end{array}$} & \multirow[b]{2}{*}{ Total } & \multicolumn{2}{|c|}{$\begin{array}{l}\text { Short term MMl's }{ }^{2} \\
\text { issued }\end{array}$} & \multicolumn{2}{|c|}{$\begin{array}{l}\text { Long term bonds } \\
\text { issued }\end{array}$} & \multirow[b]{2}{*}{ Total } & \multicolumn{2}{|c|}{ Short term loans } & \multicolumn{3}{|c|}{ Long term loans } & \multirow[b]{2}{*}{ Total } \\
\hline & & & & $\begin{array}{r}\text { Sterling } \\
\text { Treasury } \\
\text { Bills }\end{array}$ & $\begin{array}{r}\text { ECU } \\
\text { Treasury } \\
\text { Bills }\end{array}$ & $\begin{array}{r}\text { British } \\
\text { governme- } \\
\text { nt } \\
\text { securiti- } \\
\text { es }\end{array}$ & $\begin{array}{r}\text { Other } \\
\text { central } \\
\text { governme- } \\
\text { nt } \\
\text { sterling } \\
\text { bonds }\end{array}$ & & $\begin{array}{l}\text { by UK } \\
\text { MFIs }^{3}\end{array}$ & $\begin{array}{c}\text { by rest } \\
\text { of the } \\
\text { world } \\
\text { MFIs }\end{array}$ & $\begin{array}{r}\text { Finance } \\
\text { leasing }\end{array}$ & $\begin{array}{l}\text { by UK } \\
\text { residents }{ }^{4}\end{array}$ & $\begin{array}{r}\text { Other by } \\
\text { the rest } \\
\text { of the } \\
\text { world }\end{array}$ & \\
\hline & F21 & F29 & $\mathrm{F} 2$ & F33111 & F33112 & F33211 & F33212 & F3 & F411 & F419 & F423 & F424 & F429 & $\mathrm{F} 4$ \\
\hline $\begin{array}{l}2002 \\
2003 \\
2004 \\
2005 \\
2006\end{array}$ & $\begin{array}{r}\text { NIIK } \\
3.3 \\
3.4 \\
3.5 \\
3.7 \\
3.8\end{array}$ & $\begin{array}{l}\text { NIIR } \\
74.5 \\
76.7 \\
79.1 \\
84.7 \\
89.9\end{array}$ & $\begin{array}{l}\text { NIIJ } \\
77.8 \\
80.1 \\
82.7 \\
88.4 \\
93.7\end{array}$ & $\begin{array}{l}\text { NIIV } \\
21.4 \\
24.0 \\
25.0 \\
21.1 \\
19.4\end{array}$ & $\begin{array}{r}\text { NIIW } \\
- \\
- \\
- \\
- \\
-\end{array}$ & $\begin{array}{r}\text { NIJI } \\
309.3 \\
330.3 \\
370.1 \\
421.3 \\
448.4\end{array}$ & $\begin{array}{r}\text { NIJJ } \\
1.8 \\
1.6 \\
2.8 \\
3.0 \\
2.9\end{array}$ & $\begin{array}{r}\text { NIIS } \\
332.5 \\
355.9 \\
397.9 \\
445.3 \\
470.6\end{array}$ & $\begin{array}{r}\text { NIJW } \\
20.0 \\
20.8 \\
26.3 \\
28.1 \\
25.0\end{array}$ & $\begin{array}{r}\text { NIKA } \\
- \\
- \\
- \\
- \\
-\end{array}$ & $\begin{array}{r}\text { NIKJ } \\
2.3 \\
2.8 \\
3.2 \\
3.7 \\
4.0\end{array}$ & $\begin{array}{r}\text { NIKK } \\
0-\overline{1} \\
0.1 \\
0.1 \\
0.1\end{array}$ & $\begin{array}{r}\text { NIKL } \\
0.4 \\
0.2 \\
0.1 \\
0.1 \\
-\end{array}$ & $\begin{array}{l}\text { NIJU } \\
22.6 \\
23.8 \\
29.8 \\
31.9 \\
29.1\end{array}$ \\
\hline $\begin{array}{l}2007 \\
2008\end{array}$ & $\begin{array}{l}3.9 \\
3.9\end{array}$ & $\begin{array}{r}97.8 \\
118.9\end{array}$ & $\begin{array}{l}101.7 \\
122.8\end{array}$ & $\begin{array}{l}18.0 \\
31.5\end{array}$ & $\begin{array}{l}- \\
-\end{array}$ & $\begin{array}{l}490.0 \\
616.9\end{array}$ & $\begin{array}{l}2.8 \\
1.4\end{array}$ & $\begin{array}{l}510.8 \\
649.7\end{array}$ & $\begin{array}{l}24.4 \\
26.5\end{array}$ & $\begin{array}{l}- \\
-\end{array}$ & $\begin{array}{l}4.4 \\
4.5\end{array}$ & $\begin{array}{l}- \\
-\end{array}$ & $\begin{array}{l}- \\
-\end{array}$ & $\begin{array}{l}28.8 \\
31.0\end{array}$ \\
\hline 2005 Q4 & 3.7 & 84.7 & 88.4 & 21.1 & - & 421.3 & 3.0 & 445.3 & 28.1 & - & 3.7 & 0.1 & 0.1 & 31.9 \\
\hline $\begin{array}{r}2006 \text { Q1 } \\
\text { Q2 } \\
\text { Q3 } \\
\text { Q4 }\end{array}$ & $\begin{array}{l}3.7 \\
3.6 \\
3.7 \\
3.8\end{array}$ & $\begin{array}{l}83.7 \\
85.1 \\
85.8 \\
89.9\end{array}$ & $\begin{array}{l}87.3 \\
88.8 \\
89.5 \\
93.7\end{array}$ & $\begin{array}{l}19.1 \\
20.1 \\
18.9 \\
19.4\end{array}$ & $\begin{array}{l}- \\
- \\
- \\
-\end{array}$ & $\begin{array}{l}427.7 \\
434.9 \\
448.5 \\
448.4\end{array}$ & $\begin{array}{l}2.9 \\
3.0 \\
3.0 \\
2.9\end{array}$ & $\begin{array}{l}449.7 \\
458.0 \\
470.4 \\
470.6\end{array}$ & $\begin{array}{l}19.3 \\
22.8 \\
24.5 \\
25.0\end{array}$ & $\begin{array}{l}- \\
- \\
- \\
-\end{array}$ & $\begin{array}{l}3.8 \\
4.1 \\
4.0 \\
4.0\end{array}$ & $\begin{array}{l}0.1 \\
0.1 \\
0.1 \\
0.1\end{array}$ & $\begin{array}{l}0.1 \\
0.1 \\
0.1 \\
-\end{array}$ & $\begin{array}{l}23.2 \\
26.9 \\
28.6 \\
29.1\end{array}$ \\
\hline $\begin{array}{r}2007 \text { Q1 } \\
\text { Q2 } \\
\text { Q3 } \\
\text { Q4 }\end{array}$ & $\begin{array}{l}3.8 \\
3.8 \\
3.8 \\
3.9\end{array}$ & $\begin{array}{l}88.9 \\
93.1 \\
95.1 \\
97.8\end{array}$ & $\begin{array}{r}92.7 \\
96.9 \\
98.9 \\
101.7\end{array}$ & $\begin{array}{l}15.6 \\
16.8 \\
16.1 \\
18.0\end{array}$ & $\begin{array}{l}- \\
- \\
- \\
-\end{array}$ & $\begin{array}{l}444.5 \\
445.7 \\
467.9 \\
490.0\end{array}$ & $\begin{array}{l}2.9 \\
2.8 \\
2.8 \\
2.8\end{array}$ & $\begin{array}{l}463.1 \\
465.3 \\
486.8 \\
510.8\end{array}$ & $\begin{array}{l}24.4 \\
20.3 \\
19.4 \\
24.4\end{array}$ & $\begin{array}{l}- \\
- \\
- \\
-\end{array}$ & $\begin{array}{l}4.1 \\
4.1 \\
4.4 \\
4.4\end{array}$ & $\begin{array}{r}0.1 \\
- \\
- \\
-\end{array}$ & $\begin{array}{l}- \\
- \\
- \\
-\end{array}$ & $\begin{array}{l}28.5 \\
24.4 \\
23.8 \\
28.8\end{array}$ \\
\hline $\begin{array}{r}2008 \text { Q1 } \\
\text { Q2 } \\
\text { Q3 } \\
\text { Q4 }\end{array}$ & $\begin{array}{l}3.8 \\
3.8 \\
3.8 \\
3.9\end{array}$ & $\begin{array}{r}99.7 \\
107.3 \\
106.3 \\
118.9\end{array}$ & $\begin{array}{l}103.6 \\
111.2 \\
110.2 \\
122.8\end{array}$ & $\begin{array}{l}17.6 \\
20.0 \\
27.6 \\
31.5\end{array}$ & $\begin{array}{l}- \\
- \\
- \\
-\end{array}$ & $\begin{array}{l}496.7 \\
500.5 \\
531.6 \\
616.9\end{array}$ & $\begin{array}{l}2.9 \\
2.9 \\
1.4 \\
1.4\end{array}$ & $\begin{array}{l}517.2 \\
523.3 \\
560.5 \\
649.7\end{array}$ & $\begin{array}{r}16.5 \\
6.5 \\
26.1 \\
26.5\end{array}$ & $\begin{array}{l}- \\
- \\
- \\
-\end{array}$ & $\begin{array}{l}4.3 \\
4.4 \\
4.5 \\
4.5\end{array}$ & $\begin{array}{l}- \\
- \\
- \\
-\end{array}$ & $\begin{array}{l}- \\
- \\
- \\
-\end{array}$ & $\begin{array}{l}20.8 \\
10.9 \\
30.6 \\
31.0\end{array}$ \\
\hline $\begin{array}{r}2009 \text { Q1 } \\
\text { Q2 }\end{array}$ & $\begin{array}{l}3.9 \\
3.9\end{array}$ & $\begin{array}{l}135.0 \\
125.5\end{array}$ & $\begin{array}{l}139.0 \\
129.4\end{array}$ & $\begin{array}{l}43.7 \\
53.7\end{array}$ & $\begin{array}{l}- \\
-\end{array}$ & $\begin{array}{l}641.8 \\
687.2\end{array}$ & $\begin{array}{l}1.4 \\
1.4\end{array}$ & $\begin{array}{l}686.9 \\
742.3\end{array}$ & $\begin{array}{l}7.9 \\
6.4\end{array}$ & $\begin{array}{l}- \\
-\end{array}$ & $\begin{array}{l}4.6^{\dagger} \\
4.6\end{array}$ & $\begin{array}{l}- \\
-\end{array}$ & $\begin{array}{l}- \\
-\end{array}$ & $\begin{array}{l}12.5^{\dagger} \\
11.0\end{array}$ \\
\hline
\end{tabular}

\begin{tabular}{|c|c|c|c|c|}
\hline & $\begin{array}{l}\text { Other accounts } \\
\text { receivable/payable }\end{array}$ & Total financial liabilities & Total financial assets & Net assets $(+) /$ liabilities(-) \\
\hline & F7 & $\mathrm{FL}$ & FA & BF90 \\
\hline $\begin{array}{l}2002 \\
2003 \\
2004 \\
2005 \\
2006\end{array}$ & $\begin{array}{l}\text { NILG } \\
30.0 \\
32.8 \\
35.4 \\
35.0 \\
37.9\end{array}$ & $\begin{array}{r}\text { NIJT } \\
462.9 \\
492.7 \\
545.7 \\
600.6 \\
631.2\end{array}$ & $\begin{array}{l}\text { NIGP } \\
160.8 \\
159.9 \\
162.8 \\
182.4 \\
192.4\end{array}$ & $\begin{array}{r}\text { NZDZ } \\
-302.1 \\
-332.8 \\
-383.0 \\
-418.1 \\
-438.8\end{array}$ \\
\hline $\begin{array}{l}2007 \\
2008\end{array}$ & $\begin{array}{l}37.7^{\dagger} \\
35.8^{\dagger}\end{array}$ & $\begin{array}{l}679.0^{\dagger} \\
839.4^{\dagger}\end{array}$ & $\begin{array}{l}201.7^{\dagger} \\
285.1^{\dagger}\end{array}$ & $\begin{array}{l}-477.4^{\dagger} \\
-554.3^{\dagger}\end{array}$ \\
\hline 2005 Q4 & 35.0 & 600.6 & 182.4 & -418.1 \\
\hline $\begin{array}{r}2006 \text { Q1 } \\
\text { Q2 } \\
\text { Q3 } \\
\text { Q4 }\end{array}$ & $\begin{array}{l}36.9 \\
37.7 \\
38.3 \\
37.9\end{array}$ & $\begin{array}{l}597.1 \\
611.4 \\
626.8 \\
631.2\end{array}$ & $\begin{array}{l}192.4 \\
190.6 \\
194.6 \\
192.4\end{array}$ & $\begin{array}{l}-404.7 \\
-420.8 \\
-432.2 \\
-438.8\end{array}$ \\
\hline $\begin{array}{r}2007 \text { Q1 } \\
\text { Q2 } \\
\text { Q3 } \\
\text { Q4 }\end{array}$ & $\begin{array}{l}37.7 \\
38.0 \\
38.0 \\
37.7\end{array}$ & $\begin{array}{l}622.0 \\
624.5 \\
647.5 \\
679.0\end{array}$ & $\begin{array}{l}201.5 \\
195.5 \\
202.3 \\
201.7\end{array}$ & $\begin{array}{l}-420.6 \\
-429.0 \\
-445.3 \\
-477.4\end{array}$ \\
\hline $\begin{array}{r}2008 \text { Q1 } \\
\text { Q2 } \\
\text { Q3 } \\
\text { Q4 }\end{array}$ & $\begin{array}{l}37.7^{\dagger} \\
37.0 \\
36.7 \\
35.8\end{array}$ & $\begin{array}{l}679.3^{\dagger} \\
682.4 \\
738.1 \\
839.4\end{array}$ & $\begin{array}{l}209.6^{\dagger} \\
200.6 \\
244.2 \\
285.1\end{array}$ & $\begin{array}{l}-469.7^{\dagger} \\
-481.8 \\
-493.9 \\
-554.3\end{array}$ \\
\hline $\begin{array}{r}2009 \text { Q1 } \\
\text { Q2 }\end{array}$ & $\begin{array}{l}35.5 \\
36.4\end{array}$ & $\begin{array}{l}873.9 \\
919.1\end{array}$ & $\begin{array}{l}300.2 \\
297.9\end{array}$ & $\begin{array}{l}-573.7 \\
-621.2\end{array}$ \\
\hline $\begin{array}{c}1 \text { Monet } \\
2 \text { Money } \\
3 \text { All loa } \\
\text { term Io } \\
4 \text { Other } \\
\text { used f }\end{array}$ & $\begin{array}{l}\text { and all finance leas } \\
\text { ans, loans secured }\end{array}$ & $\begin{array}{l}\text { ated as long } \\
\text { gs and loans }\end{array}$ & & $\begin{array}{r}\text { Sources: National Statistics; } \\
\text { Bank of England }\end{array}$ \\
\hline
\end{tabular}




\begin{tabular}{|c|c|c|c|c|c|c|c|c|c|c|c|}
\hline & \multicolumn{11}{|c|}{ Financial assets } \\
\hline & \multicolumn{5}{|c|}{ Currency and deposits } & \multicolumn{6}{|c|}{ Securities other than shares } \\
\hline & & & & & & Sho & term MMl's ${ }^{2}$ i & ued & Long term & nds issued & \\
\hline & $\begin{array}{r}\text { Sterling } \\
\text { Bank } \\
\text { deposits }\end{array}$ & $\begin{array}{r}\text { currency } \\
\text { Bank } \\
\text { deposits }\end{array}$ & $\begin{array}{r}\text { Sterling } \\
\text { B.socs } \\
\text { deposits }\end{array}$ & $\begin{array}{l}\text { Deposits } \\
\text { other than } \\
\text { with MFI's }\end{array}$ & Total & $\begin{array}{r}\text { by UK } \\
\text { general } \\
\text { government }\end{array}$ & by UK MFI's & $\begin{array}{r}\text { by other UK } \\
\text { residents }\end{array}$ & $\begin{array}{r}\text { by UK } \\
\text { central } \\
\text { government }\end{array}$ & $\begin{array}{r}\text { by other UK } \\
\text { residents }\end{array}$ & Total \\
\hline & F2211 & $\mathrm{F} 2212$ & F2213 & F29 & $\mathrm{F} 2$ & AF3311 & F3315 & F3316 & F3321 & F3326 & F3 \\
\hline $\begin{array}{l}2002 \\
2003 \\
2004 \\
2005 \\
2006\end{array}$ & $\begin{array}{r}\text { NJEP } \\
10.2 \\
10.6 \\
13.6 \\
13.9 \\
17.6\end{array}$ & $\begin{array}{r}\text { NJEQ } \\
- \\
- \\
- \\
- \\
-\end{array}$ & $\begin{array}{r}\text { NJER } \\
5.4 \\
6.5 \\
7.8 \\
7.6 \\
9.3\end{array}$ & $\begin{array}{r}\text { NJET } \\
0.3 \\
0.4 \\
0.4 \\
0.4 \\
0.2\end{array}$ & $\begin{array}{r}\text { NJEL } \\
15.9 \\
17.6 \\
21.8 \\
21.9 \\
27.1\end{array}$ & $\begin{array}{r}\text { NJEW } \\
0.2 \\
0.2 \\
0.1 \\
0.1 \\
0.1\end{array}$ & $\begin{array}{r}\text { NJFB } \\
3.8 \\
3.9 \\
3.6 \\
3.5 \\
2.5\end{array}$ & $\begin{array}{r}\text { NJFG } \\
0.2 \\
0.2 \\
0.1 \\
0.3 \\
0.8\end{array}$ & $\begin{array}{r}\text { NJFJ } \\
0.4 \\
0.3 \\
0.2 \\
0.2 \\
0.2\end{array}$ & $\begin{array}{r}\text { E55D } \\
- \\
- \\
\overline{-} \\
0.2 \\
0.2\end{array}$ & $\begin{array}{r}\text { NJEU } \\
4.7 \\
4.5 \\
4.1 \\
4.3 \\
3.9\end{array}$ \\
\hline $\begin{array}{l}2007 \\
2008\end{array}$ & $\begin{array}{l}19.2 \\
18.7\end{array}$ & $0 . \overline{1}$ & $\begin{array}{l}11.9 \\
10.1\end{array}$ & $\begin{array}{l}0.8 \\
2.9\end{array}$ & $\begin{array}{l}32.0 \\
31.8\end{array}$ & $\begin{array}{l}0.1 \\
0.1\end{array}$ & $\begin{array}{l}1.6 \\
1.3\end{array}$ & $\begin{array}{l}1.3 \\
1.4\end{array}$ & $\begin{array}{l}0.1 \\
0.2\end{array}$ & $\begin{array}{l}0.2 \\
0.2\end{array}$ & $\begin{array}{l}3.2 \\
3.2\end{array}$ \\
\hline $\begin{array}{r}2006 \text { Q1 } \\
\text { Q2 } \\
\text { Q3 } \\
\text { Q4 }\end{array}$ & $\begin{array}{l}14.3 \\
16.3 \\
17.4 \\
17.6\end{array}$ & $\begin{array}{r}0 . \overline{1} \\
- \\
-\end{array}$ & $\begin{array}{l}6.7 \\
9.0 \\
9.7 \\
9.3\end{array}$ & $\begin{array}{l}0.3 \\
0.2 \\
0.2 \\
0.2\end{array}$ & $\begin{array}{l}21.4 \\
25.6 \\
27.4 \\
27.1\end{array}$ & $\begin{array}{l}0.3 \\
0.1 \\
0.1 \\
0.1\end{array}$ & $\begin{array}{l}2.8 \\
2.9 \\
2.7 \\
2.5\end{array}$ & $\begin{array}{l}0.4 \\
0.6 \\
0.6 \\
0.8\end{array}$ & $\begin{array}{l}0.5 \\
0.3 \\
0.1 \\
0.2\end{array}$ & $\begin{array}{l}0.2 \\
0.2 \\
0.2 \\
0.2\end{array}$ & $\begin{array}{l}4.2 \\
4.2 \\
3.8 \\
3.9\end{array}$ \\
\hline $\begin{array}{r}2007 \text { Q1 } \\
\text { Q2 } \\
\text { Q3 } \\
\text { Q4 }\end{array}$ & $\begin{array}{l}16.6 \\
19.3 \\
20.4 \\
19.2\end{array}$ & $\begin{array}{l}- \\
- \\
- \\
-\end{array}$ & $\begin{array}{r}7.2 \\
8.8 \\
10.7 \\
11.9\end{array}$ & $\begin{array}{l}0.2 \\
0.3 \\
0.5 \\
0.8\end{array}$ & $\begin{array}{l}24.1 \\
28.5 \\
31.6 \\
32.0\end{array}$ & $\begin{array}{l}0.1 \\
0.1 \\
0.1 \\
0.1\end{array}$ & $\begin{array}{l}2.0 \\
1.7 \\
1.6 \\
1.6\end{array}$ & $\begin{array}{l}0.8 \\
1.0 \\
1.3 \\
1.3\end{array}$ & $\begin{array}{l}0.1 \\
0.2 \\
0.2 \\
0.1\end{array}$ & $\begin{array}{l}0.2 \\
0.2 \\
0.2 \\
0.2\end{array}$ & $\begin{array}{l}3.2 \\
3.2 \\
3.3 \\
3.2\end{array}$ \\
\hline $\begin{array}{r}2008 \text { Q1 } \\
\text { Q2 } \\
\text { Q3 } \\
\text { Q4 }\end{array}$ & $\begin{array}{l}18.0 \\
19.4 \\
20.5 \\
18.7\end{array}$ & $\begin{array}{l}- \\
- \\
0 . \overline{1}\end{array}$ & $\begin{array}{l}10.4 \\
12.6 \\
11.9 \\
10.1\end{array}$ & $\begin{array}{l}0.7 \\
0.9 \\
1.3 \\
2.9\end{array}$ & $\begin{array}{l}29.1 \\
33.0 \\
33.7 \\
31.8\end{array}$ & $\begin{array}{l}0.1 \\
-\overline{1} \\
0.1 \\
0.1\end{array}$ & $\begin{array}{l}1.5 \\
1.5 \\
1.3 \\
1.3\end{array}$ & $\begin{array}{l}1.4 \\
1.5 \\
1.8 \\
1.4\end{array}$ & $\begin{array}{l}0.1 \\
0.2 \\
0.2 \\
0.2\end{array}$ & $\begin{array}{l}0.2 \\
0.2 \\
0.2 \\
0.2\end{array}$ & $\begin{array}{l}3.3 \\
3.4 \\
3.6 \\
3.2\end{array}$ \\
\hline $\begin{array}{r}2009 \text { Q1 } \\
\text { Q2 }\end{array}$ & $\begin{array}{l}14.9 \\
17.0\end{array}$ & $\begin{array}{l}0.1 \\
0.1\end{array}$ & $\begin{array}{l}7.1 \\
6.1\end{array}$ & $\begin{array}{l}4.2 \\
6.7\end{array}$ & $\begin{array}{l}26.2 \\
29.8\end{array}$ & $\begin{array}{l}0.1 \\
0.1\end{array}$ & $\begin{array}{l}1.3 \\
1.2\end{array}$ & $\begin{array}{l}0.9 \\
0.7\end{array}$ & $\begin{array}{l}0.2 \\
0.2\end{array}$ & $\begin{array}{l}0.2 \\
0.2\end{array}$ & $\begin{array}{l}2.6 \\
2.4\end{array}$ \\
\hline
\end{tabular}

Financial assets - continued

\begin{tabular}{|c|c|c|c|c|c|c|c|c|c|c|c|}
\hline & \multicolumn{3}{|c|}{ Loans } & \multicolumn{5}{|c|}{ Shares and other equity } & \multirow{3}{*}{$\begin{array}{l}\text { Insurance } \\
\text { technical } \\
\text { reserves } \\
\text { Prepayments } \\
\text { of insurance } \\
\text { premiums etc }\end{array}$} & \multirow{3}{*}{$\begin{array}{r}\text { Other } \\
\text { accounts } \\
\text { receivable/- } \\
\text { payable }\end{array}$} & \multirow{3}{*}{$\begin{array}{r}\text { Total } \\
\text { financial } \\
\text { assets }\end{array}$} \\
\hline & \multicolumn{2}{|c|}{ Long term loans } & \multirow[b]{2}{*}{ Total } & \multicolumn{4}{|c|}{ Excl mutual funds' shares } & \multirow[b]{2}{*}{ Total } & & & \\
\hline & $\begin{array}{r}\text { Secured on } \\
\text { dwellings }\end{array}$ & $\begin{array}{r}\text { by UK } \\
\text { residents }^{3}\end{array}$ & & $\begin{array}{r}\text { Quoted UK } \\
\text { shares }\end{array}$ & $\begin{array}{r}\text { Unquoted UK } \\
\text { shares }\end{array}$ & $\begin{array}{r}\text { Other UK } \\
\text { equity }\end{array}$ & $\begin{array}{r}\text { by UK } \\
\text { residents }\end{array}$ & & & & \\
\hline & $\mathrm{F} 422$ & F424 & $\mathrm{F} 4$ & F514 & F515 & F516 & F517 & F5 & F62 & $\mathrm{F} 7$ & FA \\
\hline $\begin{array}{l}2002 \\
2003 \\
2004 \\
2005 \\
2006\end{array}$ & $\begin{array}{r}\text { NJGH } \\
0.8 \\
1.0 \\
1.0 \\
1.3 \\
1.6\end{array}$ & $\begin{array}{r}\text { NJGM } \\
0.3 \\
0.3 \\
0.3 \\
0.2 \\
0.2\end{array}$ & $\begin{array}{r}\text { NJFW } \\
1.1 \\
1.3 \\
1.3 \\
1.5 \\
1.8\end{array}$ & $\begin{array}{r}\text { NJGT } \\
1.0 \\
1.2 \\
0.8 \\
0.8 \\
1.0\end{array}$ & $\begin{array}{r}\text { NJGU } \\
0.4 \\
0.4 \\
0.8 \\
0.8 \\
0.8\end{array}$ & $\begin{array}{r}\text { HN69 } \\
86.0 \\
95.1 \\
106.2 \\
116.7 \\
110.9\end{array}$ & $\begin{array}{r}\text { NSOE } \\
- \\
- \\
- \\
- \\
-\end{array}$ & $\begin{array}{r}\text { NJGO } \\
87.4 \\
96.7 \\
107.7 \\
118.3 \\
112.7\end{array}$ & $\begin{array}{r}\mathrm{NJHH} \\
0.9 \\
0.8 \\
0.8 \\
0.8 \\
0.8\end{array}$ & $\begin{array}{r}\text { NJHI } \\
0.3 \\
0.8 \\
0.3 \\
0.9 \\
-0.9\end{array}$ & $\begin{array}{l}\text { NJFV } \\
110.4 \\
121.7 \\
136.0 \\
147.7 \\
145.4\end{array}$ \\
\hline $\begin{array}{l}2007 \\
2008\end{array}$ & $\begin{array}{l}2.1 \\
2.9\end{array}$ & $\begin{array}{l}0.2 \\
0.2\end{array}$ & $\begin{array}{l}2.4 \\
3.1\end{array}$ & $\begin{array}{l}0.9 \\
0.3\end{array}$ & $\begin{array}{l}0.7 \\
0.7\end{array}$ & $\begin{array}{l}111.4^{\dagger} \\
113.5^{\dagger}\end{array}$ & - & $\begin{array}{l}113.0^{\dagger} \\
114.5^{\dagger}\end{array}$ & $\begin{array}{l}0.9 \\
0.8\end{array}$ & $\begin{array}{r}-1.0^{\dagger} \\
1.2^{\dagger}\end{array}$ & $\begin{array}{l}150.5 \\
154.7^{\dagger}\end{array}$ \\
\hline 2005 Q4 & 1.3 & 0.2 & 1.5 & 0.8 & 0.8 & 116.7 & - & 118.3 & 0.8 & 0.9 & 147.7 \\
\hline $\begin{array}{r}2006 \text { Q1 } \\
\text { Q2 } \\
\text { Q3 } \\
\text { Q4 }\end{array}$ & $\begin{array}{l}1.4 \\
1.4 \\
1.5 \\
1.6\end{array}$ & $\begin{array}{l}0.2 \\
0.2 \\
0.2 \\
0.2\end{array}$ & $\begin{array}{l}1.6 \\
1.6 \\
1.8 \\
1.8\end{array}$ & $\begin{array}{l}1.0 \\
1.1 \\
1.1 \\
1.0\end{array}$ & $\begin{array}{l}0.8 \\
0.8 \\
0.8 \\
0.8\end{array}$ & $\begin{array}{l}118.1 \\
117.4 \\
114.2 \\
110.9\end{array}$ & $\begin{array}{l}- \\
- \\
- \\
-\end{array}$ & $\begin{array}{l}119.9 \\
119.2 \\
116.0 \\
112.7\end{array}$ & $\begin{array}{l}0.8 \\
0.8 \\
0.8 \\
0.8\end{array}$ & $\begin{array}{r}3.8 \\
1.6 \\
0.3 \\
-0.9\end{array}$ & $\begin{array}{l}151.7 \\
153.0 \\
150.0 \\
145.4\end{array}$ \\
\hline $\begin{array}{r}2007 \text { Q1 } \\
\text { Q2 } \\
\text { Q3 } \\
\text { Q4 }\end{array}$ & $\begin{array}{l}1.7 \\
1.8 \\
2.0 \\
2.1\end{array}$ & $\begin{array}{l}0.2 \\
0.2 \\
0.3 \\
0.2\end{array}$ & $\begin{array}{l}1.9 \\
2.0 \\
2.2 \\
2.4\end{array}$ & $\begin{array}{l}1.0 \\
1.0 \\
0.9 \\
0.9\end{array}$ & $\begin{array}{l}0.8 \\
0.7 \\
0.7 \\
0.7\end{array}$ & $\begin{array}{l}107.2 \\
110.1 \\
110.8 \\
111.4\end{array}$ & $\begin{array}{l}- \\
- \\
- \\
-\end{array}$ & $\begin{array}{l}109.0 \\
111.8 \\
112.4 \\
113.0\end{array}$ & $\begin{array}{l}0.8 \\
0.8 \\
0.8 \\
0.9\end{array}$ & $\begin{array}{r}4.0 \\
1.6 \\
0.2 \\
-1.0\end{array}$ & $\begin{array}{l}143.1 \\
147.8 \\
150.6 \\
150.5\end{array}$ \\
\hline $\begin{array}{r}2008 \text { Q1 } \\
\text { Q2 } \\
\text { Q3 } \\
\text { Q4 }\end{array}$ & $\begin{array}{l}2.6 \\
2.7 \\
2.8 \\
2.9\end{array}$ & $\begin{array}{l}0.3 \\
0.2 \\
0.2 \\
0.2\end{array}$ & $\begin{array}{l}2.9 \\
3.0^{\dagger} \\
3.0 \\
3.1\end{array}$ & $\begin{array}{l}0.8 \\
0.7 \\
0.7 \\
0.3\end{array}$ & $\begin{array}{l}0.7 \\
0.7 \\
0.7 \\
0.7\end{array}$ & $\begin{array}{l}111.8 \\
113.7^{\dagger} \\
113.6 \\
113.5\end{array}$ & $\begin{array}{l}- \\
- \\
- \\
-\end{array}$ & $\begin{array}{l}113.3^{\dagger} \\
115.1^{\dagger} \\
115.0 \\
114.5\end{array}$ & $\begin{array}{l}0.8 \\
0.8 \\
0.8 \\
0.8\end{array}$ & $\begin{array}{l}6.3^{\dagger} \\
3.8 \\
2.3 \\
1.2\end{array}$ & $\begin{array}{l}155.8^{\dagger} \\
159.0 \\
158.4 \\
154.7\end{array}$ \\
\hline $\begin{array}{r}2009 \text { Q1 } \\
\text { Q2 }\end{array}$ & $\begin{array}{l}3.0 \\
2.7\end{array}$ & $\begin{array}{l}0.2 \\
0.2\end{array}$ & $\begin{array}{l}3.2 \\
2.9\end{array}$ & $0 . \overline{3}$ & $\begin{array}{l}0.7 \\
0.7\end{array}$ & $\begin{array}{l}113.3 \\
113.9\end{array}$ & - & $\begin{array}{l}114.0 \\
114.9\end{array}$ & $\begin{array}{l}0.7 \\
0.8\end{array}$ & $\begin{array}{l}6.8 \\
4.3\end{array}$ & $\begin{array}{l}153.5 \\
155.1\end{array}$ \\
\hline $\begin{array}{l}1 \text { Monet } \\
2 \text { Money } \\
3 \text { Other } \\
\text { used f }\end{array}$ & $\begin{array}{l}\text { ancial institut } \\
\text { et instrument } \\
\text { direct investm } \\
\text { nce leasing }\end{array}$ & ont loans, lo & . & 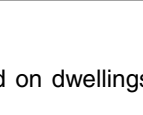 & doans & & & & & $\begin{array}{r}\text { ces: National } \\
\text { Bank }\end{array}$ & $\begin{array}{l}\text { Statistics; } \\
\text { f England }\end{array}$ \\
\hline
\end{tabular}




\begin{tabular}{|c|c|c|c|c|c|c|c|c|c|}
\hline & \multicolumn{3}{|c|}{ Securities other than shares } & \multicolumn{5}{|c|}{ Loans } & \multirow[b]{3}{*}{ Total } \\
\hline & $\begin{array}{l}\text { Short term } \\
\text { MMI's }^{1} \text { issued }\end{array}$ & $\begin{array}{l}\text { Long term bonds } \\
\text { issued }\end{array}$ & & \multicolumn{2}{|c|}{ Short term loans } & \multicolumn{3}{|c|}{ Long term loans } & \\
\hline & $\begin{array}{r}\text { by Local } \\
\text { authorities }\end{array}$ & $\begin{array}{r}\text { by UK Local } \\
\text { authorities }\end{array}$ & Total & by UK MFIs ${ }^{2,3}$ & $\begin{array}{l}\text { by rest of the } \\
\text { world MFIs }\end{array}$ & \multicolumn{2}{|r|}{ by UK residents ${ }^{4}$} & $\begin{array}{r}\text { Other by the } \\
\text { rest of the } \\
\text { world }\end{array}$ & \\
\hline & F3312 & F3322 & F3 & F411 & F419 & $\mathrm{F} 423$ & $\mathrm{~F} 424$ & $\mathrm{~F} 429$ & $\mathrm{~F} 4$ \\
\hline $\begin{array}{l}2002 \\
2003 \\
2004 \\
2005 \\
2006\end{array}$ & $\begin{array}{r}\text { NJIE } \\
- \\
- \\
- \\
- \\
-\end{array}$ & $\begin{array}{r}\text { NJIQ } \\
0.8 \\
0.8 \\
0.6 \\
0.8 \\
1.2\end{array}$ & $\begin{array}{r}\text { NJHY } \\
0.8 \\
0.8 \\
0.6 \\
0.8 \\
1.2\end{array}$ & $\begin{array}{r}\text { NJJC } \\
2.9 \\
4.3 \\
6.3 \\
7.6 \\
9.1\end{array}$ & $\begin{array}{r}\text { NJJG } \\
- \\
- \\
- \\
- \\
-\end{array}$ & $\begin{array}{r}\text { NJJP } \\
- \\
- \\
- \\
0.1 \\
0.1\end{array}$ & $\begin{array}{r}\text { NJJQ } \\
48.4 \\
44.5 \\
45.5 \\
48.0 \\
49.8\end{array}$ & $\begin{array}{r}\text { NJJR } \\
0.8 \\
1.1 \\
1.7 \\
1.9 \\
2.1\end{array}$ & $\begin{array}{r}\text { NJJA } \\
52.1 \\
49.9 \\
53.5 \\
57.5 \\
61.1\end{array}$ \\
\hline $\begin{array}{l}2007 \\
2008\end{array}$ & $\begin{array}{l}- \\
-\end{array}$ & $\begin{array}{l}1.2 \\
1.1\end{array}$ & $\begin{array}{l}1.2 \\
1.1\end{array}$ & $\begin{array}{l}10.3 \\
10.9\end{array}$ & $\begin{array}{l}- \\
-\end{array}$ & $\begin{array}{l}0.1 \\
0.1\end{array}$ & $\begin{array}{l}50.6 \\
53.9\end{array}$ & $\begin{array}{l}2.1 \\
2.5\end{array}$ & $\begin{array}{l}63.1 \\
67.4\end{array}$ \\
\hline $\begin{array}{r}2006 \text { Q1 } \\
\text { Q2 } \\
\text { Q3 } \\
\text { Q4 }\end{array}$ & $\begin{array}{l}- \\
- \\
- \\
-\end{array}$ & $\begin{array}{l}1.0 \\
1.0 \\
1.0 \\
1.2\end{array}$ & $\begin{array}{l}1.0 \\
1.0 \\
1.0 \\
1.2\end{array}$ & $\begin{array}{l}8.2 \\
8.4 \\
8.8 \\
9.1\end{array}$ & $\begin{array}{l}- \\
- \\
- \\
-\end{array}$ & $\begin{array}{l}0.1 \\
0.1 \\
0.1 \\
0.1\end{array}$ & $\begin{array}{l}49.1 \\
48.7 \\
49.7 \\
49.8\end{array}$ & $\begin{array}{l}1.9 \\
2.0 \\
2.0 \\
2.1\end{array}$ & $\begin{array}{l}59.2 \\
59.1 \\
60.5 \\
61.1\end{array}$ \\
\hline $\begin{array}{r}2007 \text { Q1 } \\
\text { Q2 } \\
\text { Q3 } \\
\text { Q4 }\end{array}$ & $\begin{array}{l}- \\
- \\
- \\
-\end{array}$ & $\begin{array}{l}1.2 \\
1.2 \\
1.2 \\
1.2\end{array}$ & $\begin{array}{l}1.2 \\
1.2 \\
1.2 \\
1.2\end{array}$ & $\begin{array}{r}9.4 \\
9.3 \\
9.7 \\
10.3\end{array}$ & $\begin{array}{l}- \\
- \\
- \\
-\end{array}$ & $\begin{array}{l}0.1 \\
0.1 \\
0.1 \\
0.1\end{array}$ & $\begin{array}{l}49.8 \\
48.6 \\
50.2 \\
50.6\end{array}$ & $\begin{array}{l}2.1 \\
2.0 \\
2.0 \\
2.1\end{array}$ & $\begin{array}{l}61.3 \\
60.0 \\
62.0 \\
63.1\end{array}$ \\
\hline $\begin{array}{r}2008 \text { Q1 } \\
\text { Q2 } \\
\text { Q3 } \\
\text { Q4 }\end{array}$ & $\begin{array}{l}- \\
- \\
- \\
-\end{array}$ & $\begin{array}{l}1.1 \\
1.1 \\
1.1 \\
1.1\end{array}$ & $\begin{array}{l}1.1 \\
1.1 \\
1.1 \\
1.1\end{array}$ & $\begin{array}{l}10.8 \\
10.7 \\
11.0 \\
10.9\end{array}$ & $\begin{array}{l}- \\
- \\
- \\
-\end{array}$ & $\begin{array}{l}0.1 \\
0.1 \\
0.1 \\
0.1\end{array}$ & $\begin{array}{l}52.4 \\
52.7 \\
53.4 \\
53.9\end{array}$ & $\begin{array}{l}2.4 \\
2.3 \\
2.4 \\
2.5\end{array}$ & $\begin{array}{l}65.6 \\
65.8 \\
66.9 \\
67.4\end{array}$ \\
\hline \multirow[t]{2}{*}{$\begin{array}{r}2009 \text { Q1 } \\
\text { Q2 }\end{array}$} & $\begin{array}{l}- \\
-\end{array}$ & $\begin{array}{l}1.0 \\
1.0\end{array}$ & $\begin{array}{l}1.0 \\
1.0\end{array}$ & $\begin{array}{l}11.4 \\
10.9\end{array}$ & $\begin{array}{l}- \\
-\end{array}$ & $\begin{array}{l}0.1 \\
0.1\end{array}$ & $\begin{array}{l}52.2^{\dagger} \\
51.5\end{array}$ & $\begin{array}{l}2.7 \\
2.8\end{array}$ & $\begin{array}{l}66.4^{\dagger} \\
65.2\end{array}$ \\
\hline & & \multicolumn{4}{|c|}{ Financial liabilities - continued } & \multicolumn{4}{|c|}{$\begin{array}{c}\text { Net financial } \\
\text { assets/liabilities } \\
\text { Net assets (+)/liabilities(-) }\end{array}$} \\
\hline $\begin{array}{l}2002 \\
2003 \\
2004 \\
2005 \\
2006\end{array}$ & & \multicolumn{2}{|c|}{$\begin{array}{r}\text { NJKM } \\
9.3 \\
9.7 \\
10.8 \\
11.0 \\
11.3\end{array}$} & \multicolumn{2}{|r|}{$\begin{array}{r}\text { NJIZ } \\
62.2 \\
60.4 \\
64.9 \\
69.3 \\
73.6\end{array}$} & \multicolumn{2}{|r|}{$\begin{array}{l}\text { NJFV } \\
110.4 \\
121.7 \\
136.0 \\
147.7 \\
145.4\end{array}$} & & $\begin{array}{r}\text { NYOJ } \\
48.2 \\
61.3 \\
71.1 \\
78.4 \\
71.8\end{array}$ \\
\hline $\begin{array}{l}2007 \\
2008\end{array}$ & & \multicolumn{2}{|c|}{$\begin{array}{l}11.0^{\dagger} \\
10.7^{\dagger}\end{array}$} & \multicolumn{2}{|r|}{$\begin{array}{l}75.3^{\dagger} \\
79.2^{\dagger}\end{array}$} & \multicolumn{2}{|r|}{$\begin{array}{l}150.5 \\
154.7^{\dagger}\end{array}$} & & $\begin{array}{l}75.2 \\
75.5^{\dagger}\end{array}$ \\
\hline 2005 Q4 & & \multicolumn{2}{|c|}{11.0} & \multicolumn{2}{|r|}{69.3} & \multicolumn{2}{|r|}{147.7} & & 78.4 \\
\hline $\begin{array}{r}2006 \text { Q1 } \\
\text { Q2 } \\
\text { Q3 } \\
\text { Q4 }\end{array}$ & & \multicolumn{2}{|c|}{$\begin{array}{l}14.2 \\
12.3 \\
12.2 \\
11.3\end{array}$} & \multicolumn{2}{|r|}{$\begin{array}{l}74.5 \\
72.4 \\
73.8 \\
73.6\end{array}$} & \multicolumn{2}{|r|}{$\begin{array}{l}151.7 \\
153.0 \\
150.0 \\
145.4\end{array}$} & & $\begin{array}{l}77.2 \\
80.6 \\
76.3 \\
71.8\end{array}$ \\
\hline $\begin{array}{r}2007 \text { Q1 } \\
\text { Q2 } \\
\text { Q3 } \\
\text { Q4 }\end{array}$ & & \multicolumn{2}{|c|}{$\begin{array}{l}14.7 \\
12.6 \\
11.8 \\
11.0\end{array}$} & & $\begin{array}{l}77.2 \\
73.7 \\
74.9 \\
75.3\end{array}$ & \multicolumn{2}{|r|}{$\begin{array}{l}143.1 \\
147.8 \\
150.6 \\
150.5\end{array}$} & & $\begin{array}{l}65.9 \\
74.1 \\
75.6 \\
75.2\end{array}$ \\
\hline $\begin{array}{r}2008 \text { Q1 } \\
\text { Q2 } \\
\text { Q3 } \\
\text { Q4 }\end{array}$ & & \multicolumn{2}{|c|}{$\begin{array}{l}14.4^{\dagger} \\
12.4^{\dagger} \\
11.5 \\
10.7\end{array}$} & & $\begin{array}{l}81.1 \\
79.4^{\dagger} \\
79.5^{\dagger} \\
79.2\end{array}$ & \multicolumn{2}{|r|}{$\begin{array}{l}155.8^{\dagger} \\
159.0 \\
158.4 \\
154.7\end{array}$} & & $\begin{array}{l}74.7^{\dagger} \\
79.7 \\
78.9 \\
75.5\end{array}$ \\
\hline $\begin{array}{r}2009 \text { Q1 } \\
\text { Q2 }\end{array}$ & & \multicolumn{2}{|c|}{$\begin{array}{l}13.9 \\
12.2\end{array}$} & \multicolumn{2}{|r|}{$\begin{array}{l}81.3 \\
78.4\end{array}$} & \multicolumn{2}{|r|}{$\begin{array}{l}153.5 \\
155.1\end{array}$} & & $\begin{array}{l}72.2 \\
76.6\end{array}$ \\
\hline
\end{tabular}

1 Money market instruments

2 All loans secured on dwellings and all finance leasing are treated as long

term loans

Bank of England

3 Monetary financial institutions

4 Other than direct investment loans, loans secured on dwellings and loans used for finance leasing. 


\begin{tabular}{|c|c|c|c|c|c|c|c|c|c|c|c|c|c|c|c|c|c|}
\hline & \multicolumn{17}{|c|}{ Financial assets } \\
\hline & \multicolumn{7}{|c|}{ Currency and deposits } & \multicolumn{9}{|c|}{ Securities other than shares } & \multirow[b]{3}{*}{ Total } \\
\hline & & & & & & & & \multicolumn{5}{|c|}{ Short term MMl's ${ }^{2}$ issued } & \multicolumn{4}{|c|}{ Bonds issued } & \\
\hline & $\begin{array}{r}\text { Curren- } \\
\text { cy }\end{array}$ & $\begin{array}{r}\text { Sterli- } \\
\text { ng Bank } \\
\text { deposi- } \\
\text { ts }\end{array}$ & $\begin{array}{r}\text { Foreign } \\
\text { curren- } \\
\text { cy Bank } \\
\text { deposi- } \\
\text { ts }\end{array}$ & $\begin{array}{r}\text { Sterli- } \\
\text { ng } \\
\text { B.socs } \\
\text { deposi- } \\
\text { ts }\end{array}$ & $\begin{array}{r}\text { ts with } \\
\text { Rest of } \\
\text { the } \\
\text { World } \\
\text { MFIs(1) }\end{array}$ & $\begin{array}{l}\text { ts } \\
\text { other } \\
\text { than } \\
\text { with } \\
\text { MFl's }\end{array}$ & Total & $\begin{array}{r}\text { by UK } \\
\text { general } \\
\text { govern- } \\
\text { ment }\end{array}$ & $\begin{array}{r}\text { UK } \\
\text { local } \\
\text { author- } \\
\text { ity } \\
\text { bills }\end{array}$ & $\begin{array}{r}\text { by UK } \\
\text { MFI's }\end{array}$ & $\begin{array}{r}\text { by } \\
\text { other } \\
\text { UK } \\
\text { reside- } \\
\text { nts }\end{array}$ & $\begin{array}{l}\text { by rest } \\
\text { of the } \\
\text { world }\end{array}$ & $\begin{array}{r}\text { by UK } \\
\text { central } \\
\text { govern- } \\
\text { ment }\end{array}$ & $\begin{array}{r}\text { by UK } \\
\text { local } \\
\text { author- } \\
\text { ities }\end{array}$ & $\begin{array}{r}\text { by } \\
\text { other } \\
\text { UK } \\
\text { reside- } \\
\text { nts }\end{array}$ & $\begin{array}{l}\text { by the } \\
\text { rest of } \\
\text { the } \\
\text { world }\end{array}$ & \\
\hline & F21 & F2211 & F2212 & $\mathrm{F} 2213$ & F229 & F29 & $\mathrm{F} 2$ & F3311 & F3312 & F3315 & F3316 & F3319 & F3321 & F3322 & F3326 & F3329 & F3 \\
\hline $\begin{array}{l}2002 \\
2003 \\
2004 \\
2005 \\
2006\end{array}$ & $\begin{array}{r}\text { NNMQ } \\
29.3 \\
31.2 \\
33.7 \\
35.6 \\
37.4\end{array}$ & $\begin{array}{l}\text { NNMT } \\
468.9 \\
510.3 \\
553.6 \\
592.1 \\
643.0\end{array}$ & $\begin{array}{r}\text { NNMU } \\
2.2 \\
2.6 \\
3.1 \\
3.4 \\
3.9\end{array}$ & $\begin{array}{l}\text { NNMV } \\
135.0 \\
143.5 \\
156.3 \\
174.8 \\
189.1\end{array}$ & $\begin{array}{r}\text { NNMW } \\
32.7 \\
40.7 \\
50.8 \\
57.7 \\
63.3\end{array}$ & $\begin{array}{r}\text { NNMX } \\
62.7 \\
67.2 \\
68.7 \\
72.9 \\
79.0\end{array}$ & $\begin{array}{r}\text { NNMP } \\
731.0 \\
795.5 \\
866.2 \\
936.5 \\
1015.8\end{array}$ & $\begin{array}{r}\text { NNNA } \\
- \\
- \\
- \\
- \\
-\end{array}$ & $\begin{array}{r}\text { NNNE } \\
- \\
- \\
- \\
- \\
-\end{array}$ & $\begin{array}{r}\text { NNNF } \\
2.3 \\
2.3 \\
2.5 \\
1.9 \\
3.1\end{array}$ & $\begin{array}{r}\text { NNNK } \\
0.5 \\
0.5 \\
0.5 \\
0.4 \\
0.5\end{array}$ & $\begin{array}{r}\text { NNNL } \\
- \\
- \\
- \\
- \\
-\end{array}$ & $\begin{array}{r}\text { NNNN } \\
40.3 \\
35.0 \\
31.0 \\
37.5 \\
18.0\end{array}$ & $\begin{array}{r}\text { NNNQ } \\
0.3 \\
0.4 \\
0.3 \\
0.4 \\
0.7\end{array}$ & $\begin{array}{r}\text { NNNU } \\
4.5 \\
4.7 \\
4.9 \\
5.1 \\
5.3\end{array}$ & $\begin{array}{r}\text { NNNV } \\
7.8 \\
7.7 \\
7.7 \\
7.7 \\
7.5\end{array}$ & $\begin{array}{r}\text { NNMY } \\
55.7 \\
50.6 \\
47.0 \\
53.0 \\
35.2\end{array}$ \\
\hline 2005 Q4 & 35.6 & 592.1 & 3.4 & 174.8 & 57.7 & 72.9 & 936.5 & - & - & 1.9 & 0.4 & - & 37.5 & 0.4 & 5.1 & 7.7 & 53.0 \\
\hline $\begin{array}{r}2006 \text { Q1 } \\
\text { Q2 } \\
\text { Q3 } \\
\text { Q4 }\end{array}$ & $\begin{array}{l}35.3 \\
35.4 \\
35.6 \\
37.4\end{array}$ & $\begin{array}{l}604.3 \\
619.2 \\
632.2 \\
643.0\end{array}$ & $\begin{array}{l}3.3 \\
3.5 \\
3.8 \\
3.9\end{array}$ & $\begin{array}{l}178.9 \\
182.6 \\
185.2 \\
189.1\end{array}$ & $\begin{array}{l}61.2 \\
60.4 \\
63.7 \\
63.3\end{array}$ & $\begin{array}{l}74.2 \\
75.4 \\
76.5 \\
79.0\end{array}$ & $\begin{array}{r}957.2 \\
976.6 \\
997.0 \\
1015.8\end{array}$ & $\begin{array}{l}- \\
- \\
- \\
-\end{array}$ & $\begin{array}{l}- \\
- \\
- \\
-\end{array}$ & $\begin{array}{l}1.8 \\
3.4 \\
3.5 \\
3.1\end{array}$ & $\begin{array}{l}0.5 \\
0.5 \\
0.5 \\
0.5\end{array}$ & $\begin{array}{l}- \\
- \\
- \\
-\end{array}$ & $\begin{array}{l}19.0 \\
14.4 \\
15.6 \\
18.0\end{array}$ & $\begin{array}{l}0.5 \\
0.5 \\
0.5 \\
0.7\end{array}$ & $\begin{array}{l}5.2 \\
5.2 \\
5.3 \\
5.3\end{array}$ & $\begin{array}{l}7.7 \\
7.6 \\
7.6 \\
7.5\end{array}$ & $\begin{array}{l}34.7 \\
31.6 \\
33.0 \\
35.2\end{array}$ \\
\hline $\begin{array}{r}2007 \text { Q1 } \\
\text { Q2 } \\
\text { Q3 } \\
\text { Q4 }\end{array}$ & $\begin{array}{l}36.4 \\
37.0 \\
37.9 \\
39.6\end{array}$ & $\begin{array}{l}655.6 \\
670.8 \\
678.7 \\
686.6\end{array}$ & $\begin{array}{l}3.9 \\
4.1 \\
4.3 \\
4.9\end{array}$ & $\begin{array}{l}193.4 \\
197.8 \\
204.1 \\
212.0\end{array}$ & $\begin{array}{l}68.3 \\
71.8 \\
72.4 \\
76.2\end{array}$ & $\begin{array}{l}79.9 \\
82.2 \\
83.3 \\
84.8\end{array}$ & $\begin{array}{ll}1 & 037.4 \\
1 & 063.7 \\
1 & 080.7 \\
1 & 104.1\end{array}$ & $\begin{array}{l}- \\
- \\
- \\
-\end{array}$ & $\begin{array}{l}- \\
- \\
- \\
-\end{array}$ & $\begin{array}{l}2.6 \\
3.3 \\
5.0 \\
4.6\end{array}$ & $\begin{array}{l}0.5 \\
0.3 \\
0.3 \\
0.4\end{array}$ & $\begin{array}{l}- \\
- \\
- \\
-\end{array}$ & $\begin{array}{r}9.8 \\
13.8 \\
13.1 \\
14.2\end{array}$ & $\begin{array}{l}0.7 \\
0.8 \\
0.8 \\
0.9\end{array}$ & $\begin{array}{l}5.4 \\
5.4 \\
5.5 \\
5.5\end{array}$ & $\begin{array}{l}7.5 \\
7.5 \\
7.5 \\
7.6\end{array}$ & $\begin{array}{l}26.5 \\
31.1 \\
32.3 \\
33.2\end{array}$ \\
\hline $\begin{array}{r}2008 \text { Q1 } \\
\text { Q2 } \\
\text { Q3 } \\
\text { Q4 }\end{array}$ & $\begin{array}{l}39.0 \\
39.6 \\
39.5 \\
42.7\end{array}$ & $\begin{array}{l}693.1 \\
706.9 \\
706.0 \\
710.9\end{array}$ & $\begin{array}{l}5.2 \\
5.3 \\
5.1 \\
6.3\end{array}$ & $\begin{array}{l}222.4 \\
227.2 \\
230.5 \\
233.9\end{array}$ & $\begin{array}{l}85.0^{\dagger} \\
82.2 \\
80.9 \\
84.0\end{array}$ & $\begin{array}{l}86.0 \\
87.0 \\
90.7 \\
96.9\end{array}$ & $\begin{array}{ll}1 & 130.7^{\dagger} \\
1 & 148.2 \\
1 & 152.7 \\
1 & 174.7\end{array}$ & $\begin{array}{l}- \\
- \\
- \\
-\end{array}$ & $\begin{array}{l}- \\
- \\
- \\
-\end{array}$ & $\begin{array}{l}3.9^{\dagger} \\
3.8 \\
3.0 \\
5.6\end{array}$ & $\begin{array}{l}0.4_{\dagger}^{\dagger} \\
-0.3 \\
-0.3\end{array}$ & $\begin{array}{l}- \\
- \\
- \\
-\end{array}$ & $\begin{array}{l}10.9^{\dagger} \\
12.2 \\
12.3 \\
10.2\end{array}$ & $\begin{array}{l}0.8 \\
0.8 \\
0.8 \\
0.9\end{array}$ & $\begin{array}{l}5.6 \\
5.6 \\
5.7 \\
5.7\end{array}$ & $\begin{array}{l}7.7 \\
7.7 \\
7.8 \\
8.5\end{array}$ & $\begin{array}{l}29.4^{\dagger} \\
30.1 \\
29.4 \\
30.6\end{array}$ \\
\hline $\begin{array}{r}2009 \text { Q1 } \\
\text { Q2 }\end{array}$ & $\begin{array}{l}43.0^{\dagger} \\
42.9^{-}\end{array}$ & $\begin{array}{l}709.2^{\dagger} \\
720.0\end{array}$ & $\begin{array}{l}5.4 \\
4.7\end{array}$ & $\begin{array}{l}237.9 \\
236.0\end{array}$ & $\begin{array}{l}77.5 \\
72.0\end{array}$ & $\begin{array}{l}99.2 \\
98.1\end{array}$ & $\begin{array}{l}1172.2 \\
1173.8\end{array}$ & $\begin{array}{l}- \\
-\end{array}$ & - & $\begin{array}{l}5.6 \\
5.3\end{array}$ & $\begin{array}{l}-0.3 \\
-0.3\end{array}$ & - & $\begin{array}{r}10.3 \\
9.6\end{array}$ & $\begin{array}{l}0.9^{\dagger} \\
0.9\end{array}$ & $\begin{array}{l}5.8 \\
5.8\end{array}$ & $\begin{array}{l}8.3 \\
8.1\end{array}$ & $\begin{array}{l}30.7 \\
29.3\end{array}$ \\
\hline
\end{tabular}

Financial assets - continued

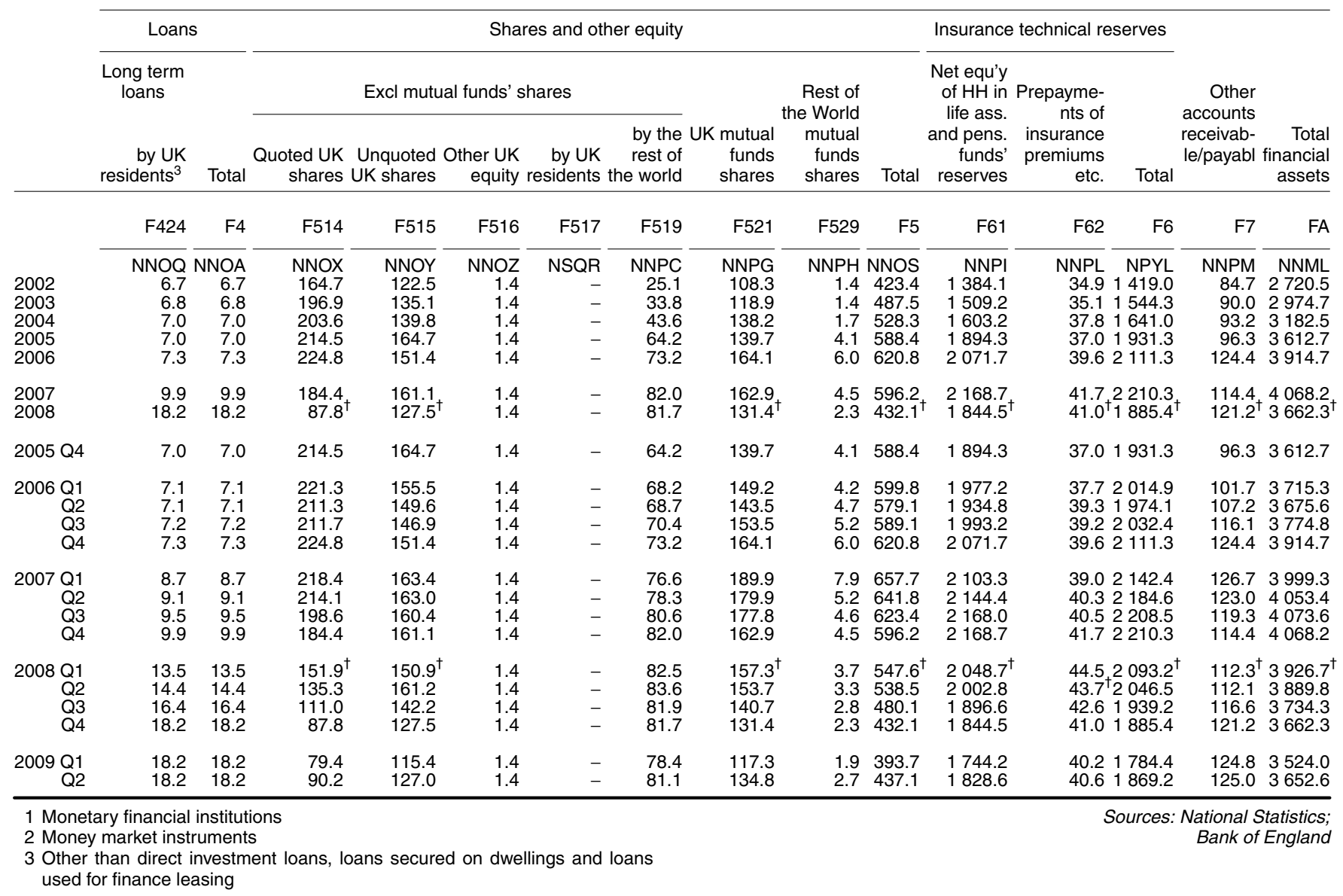




\section{$12.1 \mathrm{~N}$

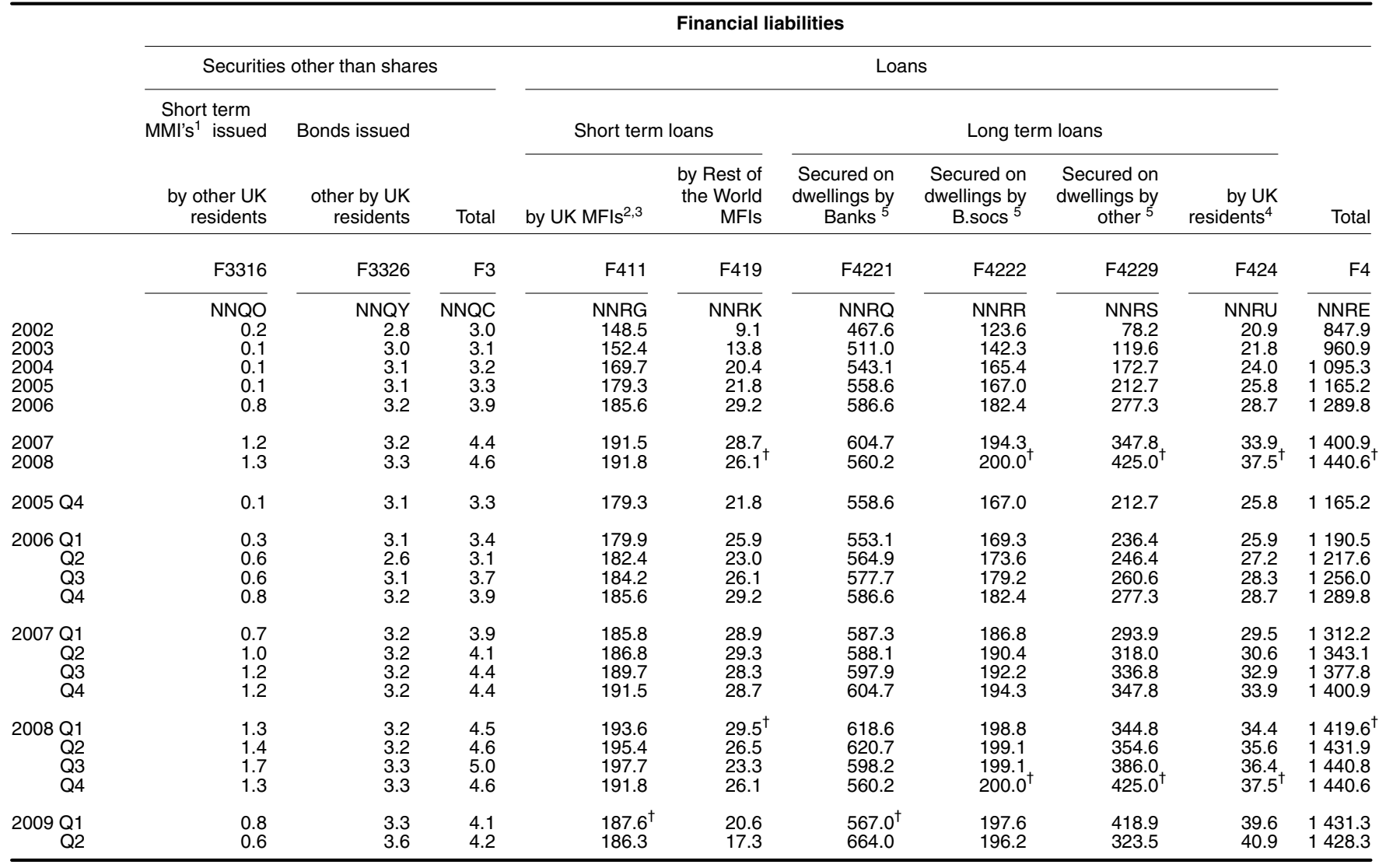

Financial liabilities - continued

assets/liabilities

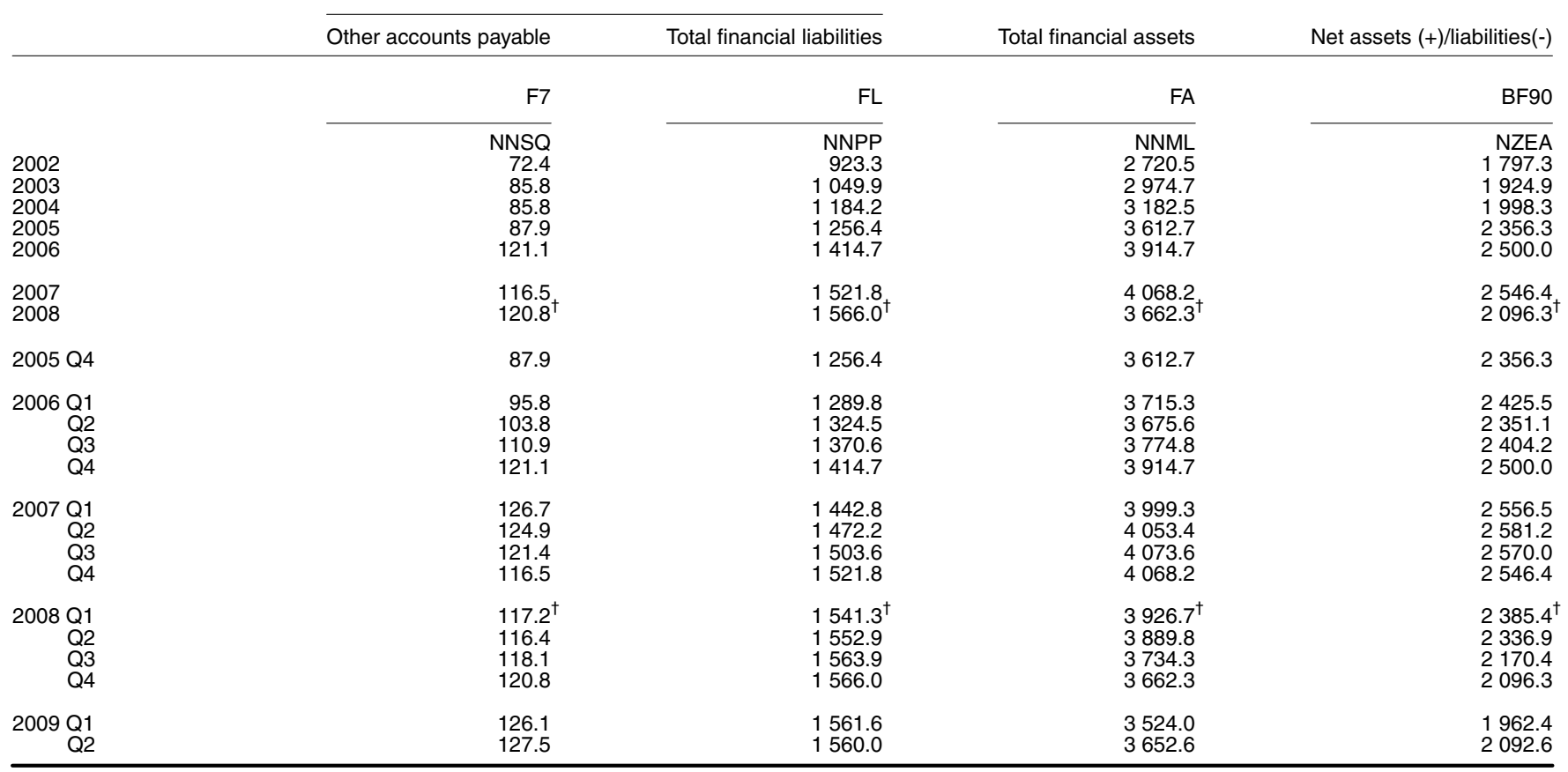

1 Money market instruments

2 All loans secured on dwellings and all finance leasing are treated as long

term loans

3 Monetary financial institutions

4 Other than direct investment loans, loans secured on dwellings and laons

for finance leasing

5 Reflects Housing Association reclassification in line with revisions policy

back to 2005 Q1. 


\begin{tabular}{|c|c|c|c|c|c|c|c|c|c|c|c|c|c|c|c|}
\hline & \multicolumn{15}{|c|}{ Financial assets } \\
\hline & \multicolumn{6}{|c|}{ Currency and deposits } & \multicolumn{9}{|c|}{ Securities other than shares } \\
\hline & \multirow[b]{2}{*}{ Currency } & \multirow[b]{2}{*}{$\begin{array}{r}\text { Sterling } \\
\text { Bank } \\
\text { deposits }\end{array}$} & \multirow[b]{2}{*}{$\begin{array}{r}\text { Foreign } \\
\text { currency } \\
\text { Bank } \\
\text { deposits }\end{array}$} & \multirow[b]{2}{*}{$\begin{array}{r}\text { Sterling } \\
\text { B.socs } \\
\text { deposits }\end{array}$} & \multirow[b]{2}{*}{$\begin{array}{r}\text { Deposits } \\
\text { other } \\
\text { than with } \\
\text { MFI's(1) }\end{array}$} & \multirow[b]{2}{*}{ Total } & \multicolumn{3}{|c|}{ Short term MMl's ${ }^{2}$ issued } & \multicolumn{5}{|c|}{ Bonds issued } & \multirow[b]{2}{*}{ Total } \\
\hline & & & & & & & $\begin{array}{r}\text { by UK } \\
\text { general } \\
\text { governme- } \\
n t\end{array}$ & $\begin{array}{l}\text { by UK } \\
\text { MFI's }\end{array}$ & $\begin{array}{r}\text { by other } \\
\text { UK } \\
\text { residents }\end{array}$ & $\begin{array}{r}\text { by UK } \\
\text { central } \\
\text { governme- } \\
\text { nt }\end{array}$ & $\begin{array}{r}\text { by UK } \\
\text { local } \\
\text { authorit- } \\
\text { ies }\end{array}$ & $\begin{array}{r}\text { Medium } \\
\text { term } \\
\text { bonds } \\
\text { issued by } \\
\text { Banks }\end{array}$ & $\begin{array}{r}\text { Medium } \\
\text { term } \\
\text { bonds } \\
\text { issued by } \\
\text { B.socs }\end{array}$ & $\begin{array}{r}\text { Other by } \\
\text { UK } \\
\text { residents }\end{array}$ & \\
\hline & $\mathrm{F} 21$ & F2211 & F2212 & F2213 & F29 & $\mathrm{F} 2$ & F3311 & F3315 & F3316 & F3321 & F3322 & F33251 & F33252 & F3326 & F3 \\
\hline $\begin{array}{l}2002 \\
2003 \\
2004 \\
2005 \\
2006\end{array}$ & $\begin{array}{r}\text { NLCW } \\
1.2 \\
1.3 \\
1.4 \\
1.4 \\
1.5\end{array}$ & $\begin{array}{l}\text { NLCZ } \\
228.0 \\
251.7 \\
279.6 \\
331.3 \\
389.0\end{array}$ & $\begin{array}{r}\text { NLDA } \\
1206.5 \\
1348.1 \\
1570.0 \\
1861.6 \\
1974.2\end{array}$ & $\begin{array}{r}\text { NLDB } \\
4.9 \\
5.4 \\
5.6 \\
6.9 \\
7.5\end{array}$ & $\begin{array}{r}\text { NLDD } \\
1.6 \\
1.9 \\
1.0 \\
0.9 \\
1.4\end{array}$ & $\begin{array}{r}\text { NLCV } \\
1442.2 \\
1608.4 \\
1857.6 \\
2202.1 \\
2373.6\end{array}$ & $\begin{array}{r}\text { NLDG } \\
0.2 \\
1.9 \\
3.8 \\
2.8 \\
3.5\end{array}$ & $\begin{array}{l}\text { NLDL } \\
140.3 \\
130.6 \\
130.7 \\
136.1 \\
162.0\end{array}$ & $\begin{array}{r}\text { NLDQ } \\
30.6 \\
23.7 \\
22.5 \\
22.2 \\
16.3\end{array}$ & $\begin{array}{r}\text { NLDT } \\
56.4 \\
66.1 \\
83.8 \\
110.7 \\
135.5\end{array}$ & $\begin{array}{r}\text { NLDW } \\
- \\
- \\
- \\
- \\
-\end{array}$ & $\begin{array}{r}\text { NLDY } \\
40.4 \\
49.5 \\
64.5 \\
85.6 \\
105.0\end{array}$ & $\begin{array}{r}\text { NLDZ } \\
3.2 \\
4.2 \\
6.4 \\
9.9 \\
9.9\end{array}$ & $\begin{array}{l}\text { NLEA } \\
228.9 \\
295.7 \\
361.8 \\
463.4 \\
530.2\end{array}$ & $\begin{array}{l}\text { NLDE } \\
499.9 \\
571.8 \\
673.6 \\
830.7 \\
962.2\end{array}$ \\
\hline 2005 Q4 & 1.4 & 331.3 & 1861.6 & 6.9 & 0.9 & 2202.1 & 2.8 & 136.1 & 22.2 & 110.7 & - & 85.6 & 9.9 & 463.4 & 830.7 \\
\hline $\begin{array}{r}2006 \text { Q1 } \\
\text { Q2 } \\
\text { Q3 } \\
\text { Q4 }\end{array}$ & $\begin{array}{l}1.4 \\
1.7 \\
1.9 \\
1.5\end{array}$ & $\begin{array}{l}344.6 \\
375.0 \\
366.5 \\
389.0\end{array}$ & $\begin{array}{ll}2 & 063.0 \\
2 & 007.4 \\
2 & 030.3 \\
1 & 974.2\end{array}$ & $\begin{array}{l}7.0 \\
7.2 \\
7.4 \\
7.5\end{array}$ & $\begin{array}{l}0.5 \\
0.4 \\
0.8 \\
1.4\end{array}$ & $\begin{array}{l}2416.6 \\
2391.7 \\
2406.9 \\
2373.6\end{array}$ & $\begin{array}{l}3.1 \\
3.3 \\
3.2 \\
3.5\end{array}$ & $\begin{array}{l}149.1 \\
139.5 \\
162.6 \\
162.0\end{array}$ & $\begin{array}{l}22.7 \\
21.3 \\
18.5 \\
16.3\end{array}$ & $\begin{array}{l}114.8 \\
115.8 \\
126.1 \\
135.5\end{array}$ & $\begin{array}{l}- \\
- \\
- \\
-\end{array}$ & $\begin{array}{r}96.1 \\
94.4 \\
100.1 \\
105.0\end{array}$ & $\begin{array}{l}9.8 \\
9.6 \\
9.6 \\
9.9\end{array}$ & $\begin{array}{l}467.7 \\
487.8 \\
510.3 \\
530.2\end{array}$ & $\begin{array}{l}863.2 \\
871.5 \\
930.4 \\
962.2\end{array}$ \\
\hline $\begin{array}{r}2007 \text { Q1 } \\
\text { Q2 } \\
\text { Q3 } \\
\text { Q4 }\end{array}$ & $\begin{array}{l}1.5 \\
1.8 \\
2.0 \\
1.5\end{array}$ & $\begin{array}{l}488.3 \\
484.4 \\
531.4 \\
604.6\end{array}$ & $\begin{array}{l}2212.1 \\
2221.8 \\
2361.0 \\
2531.0\end{array}$ & $\begin{array}{l}7.7 \\
7.6 \\
7.5 \\
7.8\end{array}$ & $\begin{array}{l}0.4 \\
0.6 \\
0.7 \\
1.1\end{array}$ & $\begin{array}{l}2710.0 \\
2716.2 \\
2902.6 \\
3146.1\end{array}$ & $\begin{array}{l}4.2 \\
4.9 \\
5.8 \\
7.2\end{array}$ & $\begin{array}{l}162.5 \\
171.0 \\
187.1 \\
183.7\end{array}$ & $\begin{array}{l}15.9 \\
12.7 \\
17.1 \\
19.1\end{array}$ & $\begin{array}{l}133.8 \\
145.9 \\
149.9 \\
158.2\end{array}$ & $\begin{array}{l}- \\
- \\
- \\
-\end{array}$ & $\begin{array}{l}116.8 \\
136.5 \\
150.1 \\
155.2\end{array}$ & $\begin{array}{l}10.6 \\
11.3 \\
12.1 \\
11.7\end{array}$ & $\begin{array}{l}534.6 \\
546.5 \\
555.0 \\
603.0\end{array}$ & $\begin{array}{rl} & 978.3 \\
1 & 028.8 \\
1 & 077.1 \\
1 & 138.2\end{array}$ \\
\hline $\begin{array}{r}2008 \text { Q1 } \\
\text { Q2 } \\
\text { Q3 } \\
\text { Q4 }\end{array}$ & $\begin{array}{l}1.6 \\
1.8 \\
2.0 \\
1.5\end{array}$ & $\begin{array}{l}613.0 \\
565.8 \\
580.3 \\
540.8\end{array}$ & 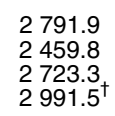 & $\begin{array}{l}7.4 \\
8.7 \\
8.1 \\
7.5\end{array}$ & $\begin{array}{l}0.3 \\
0.1 \\
0.4 \\
0.8\end{array}$ & $\begin{array}{ll}3 & 414.1 \\
3 & 036.2 \\
3 & 314.2 \\
3 & 542.2^{\dagger}\end{array}$ & $\begin{array}{c}7.9 \\
10.7^{\dagger} \\
16.6 \\
21.0\end{array}$ & $\begin{array}{l}227.5^{\dagger} \\
217.4 \\
216.9 \\
202.2\end{array}$ & $\begin{array}{l}19.1 \\
19.4 \\
22.1 \\
29.9\end{array}$ & $\begin{array}{l}162.1^{\dagger} \\
168.0 \\
191.1 \\
200.0\end{array}$ & $\begin{array}{l}- \\
- \\
- \\
-\end{array}$ & $\begin{array}{l}155.6 \\
159.6 \\
158.5 \\
188.3\end{array}$ & $\begin{array}{l}12.2 \\
11.7 \\
10.1 \\
11.9\end{array}$ & $\begin{array}{l}625.1^{\dagger} \\
685.5 \\
716.9 \\
772.9\end{array}$ & $\begin{array}{ll}1 & 209.5^{\dagger} \\
1 & 272.2 \\
1 & 332.2 \\
1 & 426.2\end{array}$ \\
\hline $\begin{array}{r}2009 \text { Q1 } \\
\text { Q2 }\end{array}$ & $\begin{array}{l}1.5 \\
1.7\end{array}$ & $\begin{array}{l}551.6^{\dagger} \\
527.7\end{array}$ & $\begin{array}{l}2809.7 \\
2421.2\end{array}$ & $\begin{array}{l}7.9 \\
8.2\end{array}$ & $\begin{array}{l}0.8 \\
0.5\end{array}$ & $\begin{array}{l}3371.5 \\
2959.4\end{array}$ & $\begin{array}{l}25.2 \\
29.8\end{array}$ & $\begin{array}{l}258.1 \\
250.0\end{array}$ & $\begin{array}{l}28.0^{\dagger} \\
23.7\end{array}$ & $\begin{array}{l}215.9 \\
207.5\end{array}$ & - & $\begin{array}{l}178.8 \\
185.8\end{array}$ & $\begin{array}{r}10.5 \\
8.9\end{array}$ & $\begin{array}{l}764.2 \\
801.6\end{array}$ & $\begin{array}{l}1480.6 \\
1507.3\end{array}$ \\
\hline
\end{tabular}

\begin{tabular}{|c|c|c|c|c|c|c|c|c|c|c|c|c|c|c|c|c|c|}
\hline & \multicolumn{17}{|c|}{ Financial assets - continued } \\
\hline & \multicolumn{7}{|c|}{ Loans } & \multicolumn{5}{|c|}{ Shares and other equity } & \multicolumn{3}{|c|}{$\begin{array}{l}\text { Insurance technical } \\
\text { reserves }\end{array}$} & \multirow[b]{3}{*}{$\begin{array}{r}\text { Other } \\
\text { accoun- } \\
\text { ts } \\
\text { receiv- } \\
\text { able/pa }\end{array}$} & \multirow[b]{3}{*}{$\begin{array}{r}\text { Total } \\
\text { financ- } \\
\text { ial } \\
\text { assets }\end{array}$} \\
\hline & $\begin{array}{l}\text { Short } \\
\text { loa }\end{array}$ & $\begin{array}{l}t \text { term } \\
\text { ans }\end{array}$ & \multicolumn{4}{|c|}{ Long term loans } & \multicolumn{4}{|c|}{$\begin{array}{l}\text { Excl mutual funds' } \\
\text { shares }\end{array}$} & \multirow{2}{*}{\multicolumn{3}{|c|}{$\begin{array}{r}\text { Net } \\
\text { equ'y } \\
\text { of HH } \\
\text { in life } \\
\text { ass. } \\
\text { and } \\
\text { pens. } \\
\text { fund } \\
\text { reserv- } \\
\text { Total } \\
\text { es }\end{array}$}} & \multirow{2}{*}{$\begin{array}{r}\text { Prepay- } \\
\text { ments } \\
\text { of } \\
\text { insura- } \\
\text { nce } \\
\text { premiu- } \\
\text { ms etc. }\end{array}$} & \multirow[b]{2}{*}{ Total } & & \\
\hline & $\begin{array}{l}\text { by rest } \\
\text { of the } \\
\text { world } \\
\text { MFl's }\end{array}$ & $\begin{array}{r}\text { by } \\
\text { non-ba- } \\
\text { nk } \\
\text { overse- } \\
\text { as }\end{array}$ & $\begin{array}{r}\text { Direct } \\
\text { invest- } \\
\text { mentloa } \\
\text { outward }\end{array}$ & $\begin{array}{r}\text { Direct } \\
\text { invest- } \\
\text { ment } \\
\text { loans } \\
\text { inward }\end{array}$ & $\begin{array}{r}\text { other } \\
\text { by UK } \\
\text { reside- } \\
\text { nts }\end{array}$ & $\begin{array}{l}\text { Other } \\
\text { by rest } \\
\text { of the } \\
\text { world }\end{array}$ & Total & $\begin{array}{r}\text { Quoted } \\
\text { UK } \\
\text { shares }\end{array}$ & $\begin{array}{l}\text { Unquot- } \\
\text { ed UK } \\
\text { shares }\end{array}$ & $\begin{array}{r}\text { Other } \\
\text { UK } \\
\text { equity }\end{array}$ & & & & & & & \\
\hline & F4191 & F4192 & F4211 & F4212 & $\mathrm{F} 424$ & $\mathrm{~F} 429$ & $\mathrm{~F} 4$ & F514 & F515 & F516 & F521 & F5 & F61 & F62 & F6 & F7 & FA \\
\hline $\begin{array}{l}2002 \\
2003 \\
2004 \\
2005 \\
2006\end{array}$ & $\begin{array}{l}\text { NLEM } \\
166.1 \\
212.0 \\
302.8 \\
343.0 \\
419.8\end{array}$ & $\begin{array}{r}\text { ZMEA } \\
280.9 \\
308.3 \\
343.8 \\
538.3 \\
466.4\end{array}$ & $\begin{array}{c}\text { NLEP } \\
128.4 \\
127.7 \\
149.0 \\
184.5 \\
194.2\end{array}$ & $\begin{array}{r}\text { NLEQ } \\
155.6 \\
152.9 \\
156.1 \\
174.2 \\
182.6\end{array}$ & $\begin{array}{r}\text { NROS } \\
7.3 \\
7.2 \\
7.3 \\
7.3 \\
5.4\end{array}$ & $\begin{array}{r}\text { NLEX } \\
2.0 \\
2.2 \\
3.2 \\
3.2 \\
3.4\end{array}$ & $\begin{array}{r}\text { NLEG } \\
733.0 \\
803.0 \\
954.9 \\
1243.2 \\
1266.4\end{array}$ & $\begin{array}{l}\text { NLFD } \\
418.7 \\
500.4 \\
550.3 \\
659.5 \\
739.2\end{array}$ & $\begin{array}{l}\text { NLFE } \\
236.5 \\
255.4 \\
273.4 \\
348.7 \\
451.9\end{array}$ & $\begin{array}{r}\text { NLFF } \\
15.9 \\
15.9 \\
17.8 \\
18.4 \\
20.2\end{array}$ & $\begin{array}{r}\text { NLFM } \\
0.9 \\
1.0 \\
1.2 \\
1.5 \\
1.7\end{array}$ & $\begin{array}{r}\text { NLEY } \\
671.9 \\
772.8 \\
842.6 \\
1028.0 \\
1213.0\end{array}$ & $\begin{array}{r}\text { NLFO } \\
0.2 \\
0.2 \\
0.2 \\
0.2 \\
0.2\end{array}$ & $\begin{array}{r}\text { NLFR } \\
12.6 \\
10.2 \\
11.5 \\
14.2 \\
18.6\end{array}$ & $\begin{array}{r}\text { NPYF } \\
12.9 \\
10.4 \\
11.7 \\
14.4 \\
18.8\end{array}$ & $\begin{array}{r}\text { NLFS } \\
2.0 \\
1.9 \\
1.7 \\
2.0 \\
1.9\end{array}$ & $\begin{array}{rl} & \text { NLEF } \\
3 & 361.9 \\
3 & 768.3 \\
4 & 342.1 \\
5 & 320.4 \\
5 & 836.0\end{array}$ \\
\hline $\begin{array}{l}2007 \\
2008\end{array}$ & $\begin{array}{l}416.3^{\dagger} \\
351.9^{\dagger}\end{array}$ & $\begin{array}{l}536.9 \\
433.9^{\dagger}\end{array}$ & $\begin{array}{l}199.0 \\
270.4\end{array}$ & $\begin{array}{l}157.5 \\
162.9\end{array}$ & $\begin{array}{l}5.2 \\
7.0\end{array}$ & $\begin{array}{l}3.3 \\
3.9\end{array}$ & $\begin{array}{l}1313.0^{\dagger} \\
1223.0^{\dagger}\end{array}$ & $\begin{array}{l}775.1^{\dagger} \\
509.8^{\dagger}\end{array}$ & $\begin{array}{l}528.4 \\
579.6\end{array}$ & $\begin{array}{l}24.8 \\
24.7\end{array}$ & $\begin{array}{l}1.7 \\
1.0\end{array}$ & $\begin{array}{l}1330.0^{\dagger} \\
1115.2^{\dagger}\end{array}$ & $\begin{array}{l}0.2 \\
0.2\end{array}$ & $\begin{array}{r}14.4 \\
9.2\end{array}$ & $\begin{array}{r}14.6 \\
9.4\end{array}$ & $\begin{array}{l}2.2 \\
2.6\end{array}$ & $\begin{array}{l}6944.1 \\
7318.6^{\dagger}\end{array}$ \\
\hline 2005 Q4 & 343.0 & 538.3 & 184.5 & 174.2 & 7.3 & 3.2 & 1243.2 & 659.5 & 348.7 & 18.4 & 1.5 & 1028.0 & 0.2 & 14.2 & 14.4 & 2.0 & 5320.4 \\
\hline $\begin{array}{r}2006 \text { Q1 } \\
\text { Q2 } \\
\text { Q3 } \\
\text { Q4 }\end{array}$ & $\begin{array}{l}408.8 \\
369.7 \\
378.2 \\
419.8\end{array}$ & $\begin{array}{l}555.8 \\
492.3 \\
475.4 \\
466.4\end{array}$ & $\begin{array}{l}182.1 \\
192.9 \\
207.9 \\
194.2\end{array}$ & $\begin{array}{l}179.0 \\
182.4 \\
182.5 \\
182.6\end{array}$ & $\begin{array}{l}6.9 \\
6.3 \\
5.6 \\
5.4\end{array}$ & $\begin{array}{l}3.2 \\
3.4 \\
3.3 \\
3.4\end{array}$ & $\begin{array}{ll}1 & 328.9 \\
1 & 240.7 \\
1 & 247.3 \\
1 & 266.4\end{array}$ & $\begin{array}{l}707.9 \\
685.6 \\
705.4 \\
739.2\end{array}$ & $\begin{array}{l}417.3 \\
424.4 \\
446.2 \\
451.9\end{array}$ & $\begin{array}{l}18.7 \\
19.1 \\
19.9 \\
20.2\end{array}$ & $\begin{array}{l}1.7 \\
1.6 \\
1.6 \\
1.7\end{array}$ & $\begin{array}{ll}1 & 145.6 \\
1 & 130.7 \\
1 & 173.1 \\
1 & 213.0\end{array}$ & $\begin{array}{l}0.2 \\
0.2 \\
0.2 \\
0.2\end{array}$ & $\begin{array}{l}15.5 \\
16.7 \\
17.7 \\
18.6\end{array}$ & $\begin{array}{l}15.7 \\
17.0 \\
18.0 \\
18.8\end{array}$ & $\begin{array}{l}1.9 \\
2.1 \\
2.1 \\
1.9\end{array}$ & $\begin{array}{l}5771.9 \\
5653.8 \\
5777.8 \\
5836.0\end{array}$ \\
\hline $\begin{array}{r}2007 \text { Q1 } \\
\text { Q2 } \\
\text { Q3 } \\
\text { Q4 }\end{array}$ & $\begin{array}{l}434.6 \\
440.6 \\
411.2 \\
416.3\end{array}$ & $\begin{array}{l}533.2 \\
566.5 \\
568.5 \\
536.9\end{array}$ & $\begin{array}{l}192.1 \\
189.0 \\
198.9 \\
199.0\end{array}$ & $\begin{array}{l}158.6 \\
156.1 \\
150.7 \\
157.5\end{array}$ & $\begin{array}{l}5.4 \\
5.3 \\
5.2 \\
5.2\end{array}$ & $\begin{array}{l}3.3 \\
3.2 \\
3.3 \\
3.3\end{array}$ & $\begin{array}{l}1321.8 \\
1355.4 \\
1332.7 \\
1313.0\end{array}$ & $\begin{array}{l}766.0 \\
793.0 \\
762.2 \\
775.1\end{array}$ & $\begin{array}{l}430.2 \\
482.6 \\
505.8 \\
528.4\end{array}$ & $\begin{array}{l}21.0 \\
23.4 \\
24.6 \\
24.8\end{array}$ & $\begin{array}{l}1.8 \\
1.8 \\
1.8 \\
1.7\end{array}$ & $\begin{array}{ll}1 & 219.0 \\
1 & 300.8 \\
1 & 294.3 \\
1 & 330.0\end{array}$ & $\begin{array}{l}0.2 \\
0.2 \\
0.2 \\
0.2\end{array}$ & $\begin{array}{l}17.1 \\
16.3 \\
15.2 \\
14.4\end{array}$ & $\begin{array}{l}17.3 \\
16.5 \\
15.5 \\
14.6\end{array}$ & $\begin{array}{l}1.9 \\
2.2 \\
2.2 \\
2.2\end{array}$ & $\begin{array}{l}6248.3 \\
6419.9 \\
6624.3 \\
6944.1\end{array}$ \\
\hline $\begin{array}{r}2008 \text { Q1 } \\
\text { Q2 } \\
\text { Q3 } \\
\text { Q4 }\end{array}$ & $\begin{array}{l}430.7^{\dagger} \\
372.3 \\
339.1 \\
351.9\end{array}$ & $\begin{array}{l}641.4^{\dagger} \\
558.0 \\
500.8 \\
433.9\end{array}$ & $\begin{array}{l}207.0 \\
208.9 \\
228.3 \\
270.4\end{array}$ & $\begin{array}{l}159.9 \\
157.5 \\
153.7 \\
162.9\end{array}$ & $\begin{array}{l}5.1 \\
5.3 \\
5.6 \\
7.0\end{array}$ & $\begin{array}{l}3.7 \\
3.6 \\
3.7 \\
3.9\end{array}$ & $\begin{array}{ll}1 & 442.7^{\dagger} \\
1 & 300.3 \\
1 & 225.6 \\
1 & 223.0\end{array}$ & $\begin{array}{l}670.4^{\dagger} \\
654.1 \\
574.6 \\
509.8\end{array}$ & $\begin{array}{l}548.2 \\
564.1 \\
567.6 \\
579.6\end{array}$ & $\begin{array}{l}25.9 \\
25.7 \\
25.3 \\
24.7\end{array}$ & $\begin{array}{l}1.6 \\
1.5 \\
1.2 \\
1.0\end{array}$ & $\begin{array}{ll}1 & 246.1^{\dagger} \\
1 & 245.5 \\
1 & 168.7 \\
1 & 115.2\end{array}$ & $\begin{array}{l}0.2 \\
0.2 \\
0.2 \\
0.2\end{array}$ & $\begin{array}{l}9.9 \\
9.8 \\
9.5 \\
9.2\end{array}$ & $\begin{array}{r}10.2 \\
10.0 \\
9.7 \\
9.4\end{array}$ & $\begin{array}{l}2.4 \\
2.3 \\
2.3 \\
2.6\end{array}$ & $\begin{array}{l}7325.0^{\dagger} \\
6866.5 \\
7052.7 \\
7318.6\end{array}$ \\
\hline $\begin{array}{r}2009 \text { Q1 } \\
\text { Q2 }\end{array}$ & $\begin{array}{l}288.5 \\
247.1\end{array}$ & $\begin{array}{l}374.2 \\
368.9\end{array}$ & $\begin{array}{l}245.9^{\dagger} \\
228.8\end{array}$ & $\begin{array}{l}169.2^{\dagger} \\
161.6\end{array}$ & $\begin{array}{l}6.9 \\
6.2\end{array}$ & $\begin{array}{l}4.1 \\
4.1\end{array}$ & $\begin{array}{l}1081.9 \\
1010.4\end{array}$ & $\begin{array}{l}476.2 \\
551.4\end{array}$ & $\begin{array}{l}589.9^{\dagger} \\
589.9\end{array}$ & $\begin{array}{l}24.1 \\
24.3\end{array}$ & $\begin{array}{l}1.0 \\
1.1\end{array}$ & $\begin{array}{l}1091.2 \\
1166.6\end{array}$ & $\begin{array}{l}0.2 \\
0.2\end{array}$ & $\begin{array}{l}9.0^{\dagger} \\
9.1\end{array}$ & $\begin{array}{l}9.2^{\dagger} \\
9.3\end{array}$ & $\begin{array}{l}2.8 \\
2.9\end{array}$ & $\begin{array}{l}7037.1 \\
6655.9\end{array}$ \\
\hline
\end{tabular}




\begin{tabular}{|c|c|c|c|c|c|c|c|c|c|c|c|c|c|c|}
\hline & \multicolumn{14}{|c|}{ Financial liabilities } \\
\hline & \multicolumn{3}{|c|}{ Currency and deposits } & \multicolumn{3}{|c|}{ Securities other than shares } & \multicolumn{7}{|c|}{ Loans } & \multirow[b]{3}{*}{ Total } \\
\hline & & & & $\begin{array}{c}\text { Short } \\
\text { term } \\
\text { MMl's }^{2} \\
\text { issued }\end{array}$ & $\begin{array}{l}\text { Long term } \\
\text { bonds } \\
\text { issued }\end{array}$ & & \multicolumn{3}{|c|}{ Short term loans } & \multicolumn{4}{|c|}{ Long term loans } & \\
\hline & Currency & $\begin{array}{l}\text { with rest } \\
\text { of the } \\
\text { world } \\
\text { MFl's }{ }^{1}\end{array}$ & Total & $\begin{array}{r}\text { by the } \\
\text { Rest of } \\
\text { the World }\end{array}$ & $\begin{array}{r}\text { by the } \\
\text { Rest of } \\
\text { the World }\end{array}$ & Total & $\begin{array}{r}\text { Sterling } \\
\text { loans By } \\
\text { UK MFIs }^{3}\end{array}$ & $\begin{array}{l}\text { Foreign } \\
\text { currency } \\
\text { loans by } \\
\text { UK MFIs }\end{array}$ & $\begin{array}{l}\text { Sterling } \\
\text { loans by } \\
\text { B.socs }\end{array}$ & $\begin{array}{r}\text { Direct } \\
\text { investme- } \\
\text { nt loans } \\
\text { outward }\end{array}$ & $\begin{array}{r}\text { Direct } \\
\text { investme- } \\
\text { nt loans } \\
\text { inward }\end{array}$ & $\begin{array}{l}\text { Finance } \\
\text { leasing }\end{array}$ & $\begin{array}{l}\text { by UK } \\
\text { residents }{ }^{4}\end{array}$ & \\
\hline & F21 & F229 & $\mathrm{F} 2$ & F3319 & F3329 & F3 & F4111 & F4112 & F4113 & F4211 & F4212 & $\mathrm{F} 423$ & F424 & $\mathrm{F} 4$ \\
\hline $\begin{array}{l}2002 \\
2003 \\
2004 \\
2005 \\
2006\end{array}$ & $\begin{array}{r}\text { NLGA } \\
0.5 \\
0.6 \\
0.6 \\
0.6 \\
0.7\end{array}$ & $\begin{array}{r}\text { NLGG } \\
1203.3 \\
1399.9 \\
1605.3 \\
2055.5 \\
2189.5\end{array}$ & $\begin{array}{rr} & \text { NLFZ } \\
1 & 203.9 \\
1 & 400.5 \\
1 & 605.9 \\
2 & 056.1 \\
2 & 190.2\end{array}$ & $\begin{array}{r}\text { NLGV } \\
48.7 \\
62.0 \\
58.3 \\
63.8 \\
75.5\end{array}$ & $\begin{array}{l}\text { NLHF } \\
538.2 \\
550.1 \\
611.3 \\
717.2 \\
798.5\end{array}$ & $\begin{array}{l}\text { NLGI } \\
587.1 \\
612.1 \\
669.7 \\
781.3 \\
874.0\end{array}$ & $\begin{array}{r}\text { NLHN } \\
37.4 \\
40.2 \\
47.4 \\
66.9 \\
87.7\end{array}$ & $\begin{array}{l}\mathrm{NLHO} \\
290.9 \\
358.3 \\
448.9 \\
575.1 \\
621.3\end{array}$ & $\begin{array}{r}\text { NLHP } \\
- \\
- \\
- \\
- \\
-\end{array}$ & $\begin{array}{l}\text { NLHT } \\
114.5 \\
116.3 \\
144.5 \\
145.6 \\
147.2\end{array}$ & $\begin{array}{r}\text { NLHU } \\
61.6 \\
59.0 \\
61.0 \\
77.0 \\
76.9\end{array}$ & $\begin{array}{r}\text { NLHZ } \\
- \\
- \\
- \\
- \\
-\end{array}$ & $\begin{array}{r}\text { NROS } \\
7.3 \\
7.2 \\
7.3 \\
7.3 \\
5.4\end{array}$ & $\begin{array}{l}\text { NLHK } \\
511.8 \\
580.9 \\
709.2 \\
872.0 \\
938.6\end{array}$ \\
\hline $\begin{array}{l}2007 \\
2008\end{array}$ & $\begin{array}{l}0.8 \\
0.9\end{array}$ & $\begin{array}{l}2773.7 \\
3050.5^{\dagger}\end{array}$ & $\begin{array}{l}2774.5 \\
3051.4^{\dagger}\end{array}$ & $\begin{array}{l}76.4^{\dagger} \\
73.5^{\dagger}\end{array}$ & $\begin{array}{r}905.3^{\dagger} \\
1171.1^{\dagger}\end{array}$ & $\begin{array}{r}981.7 \\
1244.6^{\dagger}\end{array}$ & $\begin{array}{l}113.1 \\
116.2\end{array}$ & $\begin{array}{r}842.8 \\
1010.0\end{array}$ & $1 . \overline{6}^{\dagger}$ & $\begin{array}{l}189.3 \\
272.3\end{array}$ & $\begin{array}{l}78.0 \\
99.7\end{array}$ & $\begin{array}{l}- \\
-\end{array}$ & $\begin{array}{l}5.2 \\
7.0\end{array}$ & $\begin{array}{l}1228.4 \\
1506.8^{\dagger}\end{array}$ \\
\hline 2005 Q4 & 0.6 & 2055.5 & 2056.1 & 63.8 & 717.2 & 781.3 & 66.9 & 575.1 & - & 145.6 & 77.0 & - & 7.3 & 872.0 \\
\hline $\begin{array}{r}2006 \text { Q1 } \\
\text { Q2 } \\
\text { Q3 } \\
\text { Q4 }\end{array}$ & $\begin{array}{l}0.8 \\
1.0 \\
0.7 \\
0.7\end{array}$ & $\begin{array}{l}2300.5 \\
2215.6 \\
2233.6 \\
2189.5\end{array}$ & $\begin{array}{l}2301.3 \\
2216.5 \\
2234.4 \\
2190.2\end{array}$ & $\begin{array}{l}72.5 \\
76.8 \\
73.9 \\
75.5\end{array}$ & $\begin{array}{l}743.2 \\
741.2 \\
745.6 \\
798.5\end{array}$ & $\begin{array}{l}815.7 \\
818.1 \\
819.5 \\
874.0\end{array}$ & $\begin{array}{l}70.7 \\
78.6 \\
80.7 \\
87.7\end{array}$ & $\begin{array}{l}636.1 \\
597.4 \\
636.9 \\
621.3\end{array}$ & $\begin{array}{l}- \\
- \\
- \\
-\end{array}$ & $\begin{array}{l}151.2 \\
139.5 \\
144.0 \\
147.2\end{array}$ & $\begin{array}{l}74.5 \\
76.6 \\
77.9 \\
76.9\end{array}$ & $\begin{array}{l}- \\
- \\
- \\
-\end{array}$ & $\begin{array}{l}6.9 \\
6.3 \\
5.6 \\
5.4\end{array}$ & $\begin{array}{l}939.4 \\
898.4 \\
945.1 \\
938.6\end{array}$ \\
\hline $\begin{array}{r}2007 \text { Q1 } \\
\text { Q2 } \\
\text { Q3 } \\
\text { Q4 }\end{array}$ & $\begin{array}{l}0.7 \\
1.0 \\
0.7 \\
0.8\end{array}$ & $\begin{array}{ll}2 & 467.5 \\
2 & 491.9 \\
2 & 596.6 \\
2 & 773.7\end{array}$ & 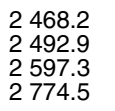 & $\begin{array}{l}84.0 \\
86.8 \\
78.4 \\
76.4\end{array}$ & $\begin{array}{l}836.1 \\
858.9 \\
865.3 \\
905.3\end{array}$ & $\begin{array}{l}920.1 \\
945.6 \\
943.8 \\
981.7\end{array}$ & $\begin{array}{r}95.6 \\
101.1 \\
111.0 \\
113.1\end{array}$ & $\begin{array}{l}712.1 \\
741.0 \\
803.0 \\
842.8\end{array}$ & $\begin{array}{l}- \\
- \\
- \\
-\end{array}$ & $\begin{array}{l}156.5 \\
154.2 \\
164.8 \\
189.3\end{array}$ & $\begin{array}{l}68.4 \\
72.4 \\
76.0 \\
78.0\end{array}$ & $\begin{array}{l}- \\
- \\
- \\
-\end{array}$ & $\begin{array}{l}5.4 \\
5.3 \\
5.2 \\
5.2\end{array}$ & $\begin{array}{ll}1 & 038.0 \\
1 & 074.0 \\
1 & 160.0 \\
1 & 228.4\end{array}$ \\
\hline $\begin{array}{r}2008 \text { Q1 } \\
\text { Q2 } \\
\text { Q3 } \\
\text { Q4 }\end{array}$ & $\begin{array}{l}0.9 \\
1.1 \\
0.7 \\
0.9\end{array}$ & $\begin{array}{l}3104.9^{\dagger} \\
2774.3 \\
2906.5 \\
3050.5\end{array}$ & $\begin{array}{l}3105.8^{\dagger} \\
2775.4 \\
2907.2 \\
3051.4\end{array}$ & $\begin{array}{l}80.3 \\
78.2^{\dagger} \\
83.1^{\dagger} \\
73.5\end{array}$ & $\begin{array}{rl} & 945.2^{\dagger} \\
& 947.6 \\
1 & 014.6 \\
1 & 171.1\end{array}$ & $\begin{array}{ll}1 & 025.7^{\dagger} \\
1 & 025.9 \\
1 & 097.7 \\
1 & 244.6\end{array}$ & $\begin{array}{l}117.9 \\
113.2 \\
118.2 \\
116.2\end{array}$ & $\begin{array}{r}940.1 \\
851.3 \\
923.8 \\
1010.0\end{array}$ & $\begin{array}{l}1.4^{\dagger} \\
1.6 \\
1.6 \\
1.6\end{array}$ & $\begin{array}{l}189.2 \\
196.0 \\
213.5 \\
272.3\end{array}$ & $\begin{array}{l}85.6 \\
88.6 \\
92.4 \\
99.7\end{array}$ & $\begin{array}{l}- \\
- \\
- \\
-\end{array}$ & $\begin{array}{l}5.1 \\
5.3 \\
5.6 \\
7.0\end{array}$ & $\begin{array}{l}1339.4^{\dagger} \\
1256.1 \\
1355.1 \\
1506.8\end{array}$ \\
\hline $\begin{array}{r}2009 \text { Q1 } \\
\text { Q2 }\end{array}$ & $\begin{array}{l}0.8 \\
0.9\end{array}$ & $\begin{array}{l}2898.0 \\
2642.9\end{array}$ & $\begin{array}{l}2898.7 \\
2643.8\end{array}$ & $\begin{array}{l}77.3 \\
81.5\end{array}$ & $\begin{array}{l}1209.9 \\
1129.6\end{array}$ & $\begin{array}{l}1287.2 \\
1211.1\end{array}$ & $\begin{array}{l}117.1 \\
104.2\end{array}$ & $\begin{array}{l}943.4 \\
812.6\end{array}$ & $\begin{array}{l}1.6 \\
1.6\end{array}$ & $\begin{array}{l}248.4^{\dagger} \\
231.6\end{array}$ & $\begin{array}{c}105.6^{\dagger} \\
99.1\end{array}$ & $\begin{array}{l}- \\
-\end{array}$ & $\begin{array}{l}6.9 \\
6.2\end{array}$ & $\begin{array}{l}1422.9 \\
1255.2\end{array}$ \\
\hline
\end{tabular}

Shares and other equity

Excl mutual funds' shares

\begin{tabular}{|c|c|c|c|c|c|c|c|}
\hline & $\begin{array}{r}\text { by the rest of the } \\
\text { world }\end{array}$ & Other UK equity & Total & $\begin{array}{l}\text { Other accounts } \\
\text { receivable/payable }\end{array}$ & $\begin{array}{r}\text { Total financial } \\
\text { liabilities }\end{array}$ & $\begin{array}{r}\text { Total financial } \\
\text { assets }\end{array}$ & $\begin{array}{r}\text { Net assets } \\
(+) / \text { liabilities(-) }\end{array}$ \\
\hline & F519 & F52 & F5 & F7 & $\mathrm{FL}$ & FA & BF90 \\
\hline $\begin{array}{l}2002 \\
2003 \\
2004 \\
2005 \\
2006\end{array}$ & $\begin{array}{rr} & \text { NLIM } \\
931.3 \\
1 & 049.0 \\
1 & 128.8 \\
1 & 348.3 \\
1 & 467.4\end{array}$ & $\begin{array}{r}\text { NLIP } \\
1.4 \\
1.4 \\
1.7 \\
4.1 \\
6.0\end{array}$ & $\begin{array}{rr} & \text { NLIC } \\
932.7 \\
1050.4 \\
1130.5 \\
1352.4 \\
1473.4\end{array}$ & $\begin{array}{r}\text { NLIW } \\
4.1 \\
4.8 \\
3.6 \\
2.7 \\
3.8\end{array}$ & $\begin{array}{rr} & \text { NLHJ } \\
3 & 239.6 \\
3 & 648.6 \\
4 & 119.0 \\
5 & 064.6 \\
5 & 480.0\end{array}$ & $\begin{array}{rr} & \text { NLEF } \\
3 & 361.9 \\
3 & 768.3 \\
4 & 342.1 \\
5 & 320.4 \\
5 & 836.0\end{array}$ & $\begin{array}{l}\text { NLFK } \\
122.4 \\
119.7 \\
223.2 \\
255.7 \\
356.0\end{array}$ \\
\hline $\begin{array}{l}2007 \\
2008\end{array}$ & $\begin{array}{l}1662.6^{\dagger} \\
1627.1^{\dagger}\end{array}$ & $\begin{array}{l}4.5 \\
2.3\end{array}$ & $\begin{array}{l}1667.1 \\
1629.4^{\dagger}\end{array}$ & $\begin{array}{l}5.6 \\
6.7\end{array}$ & $\begin{array}{l}6657.3^{\dagger} \\
7438.9^{\dagger}\end{array}$ & $\begin{array}{l}6944.1 \\
7318.6^{\dagger}\end{array}$ & $\begin{array}{r}286.9 \\
-120.3^{\dagger}\end{array}$ \\
\hline 2005 Q4 & 1348.3 & 4.1 & 1352.4 & 2.7 & 5064.6 & 5320.4 & 255.7 \\
\hline $\begin{array}{r}2006 \text { Q1 } \\
\text { Q2 } \\
\text { Q3 } \\
\text { Q4 }\end{array}$ & $\begin{array}{ll}1 & 407.2 \\
1 & 392.0 \\
1 & 426.5 \\
1 & 467.4\end{array}$ & $\begin{array}{l}4.2 \\
4.7 \\
5.2 \\
6.0\end{array}$ & $\begin{array}{ll}1 & 411.4 \\
1 & 396.7 \\
1 & 431.7 \\
1 & 473.4\end{array}$ & $\begin{array}{l}3.0 \\
3.3 \\
3.1 \\
3.8\end{array}$ & $\begin{array}{l}5470.8 \\
5532.9 \\
5433.8 \\
5480.0\end{array}$ & $\begin{array}{l}5771.9 \\
5653.8 \\
5777.8 \\
5836.0\end{array}$ & $\begin{array}{l}301.1 \\
320.9 \\
344.0 \\
356.0\end{array}$ \\
\hline $\begin{array}{r}2007 \text { Q1 } \\
\text { Q2 } \\
\text { Q3 } \\
\text { Q4 }\end{array}$ & $\begin{array}{ll}1 & 517.1 \\
1 & 586.3 \\
1 & 625.3 \\
1 & 662.6\end{array}$ & $\begin{array}{l}7.9 \\
5.2 \\
4.6 \\
4.5\end{array}$ & $\begin{array}{ll}1 & 525.1 \\
1 & 591.5 \\
1 & 629.9 \\
1 & 667.1\end{array}$ & $\begin{array}{l}4.0 \\
3.8 \\
4.1 \\
5.6\end{array}$ & $\begin{array}{ll}5 & 955.4 \\
6 & 107.7 \\
6 & 335.1 \\
6 & 657.3\end{array}$ & $\begin{array}{l}6248.3 \\
6419.9 \\
6624.3 \\
6044.1\end{array}$ & $\begin{array}{l}292.9 \\
312.2 \\
289.2 \\
286.9\end{array}$ \\
\hline $\begin{array}{r}2008 \text { Q1 } \\
\text { Q2 } \\
\text { Q3 } \\
\text { Q4 }\end{array}$ & $\begin{array}{ll}1 & 632.0^{\dagger} \\
1 & 630.3 \\
1 & 618.2 \\
1 & 627.1\end{array}$ & $\begin{array}{l}3.7 \\
3.3 \\
2.8 \\
2.3\end{array}$ & $\begin{array}{ll}1 & 635.7^{\dagger} \\
1 & 633.6 \\
1 & 621.1 \\
1 & 629.4\end{array}$ & $\begin{array}{l}5.1 \\
4.9 \\
5.5 \\
6.7\end{array}$ & $\begin{array}{l}7111.6^{\dagger} \\
6696.0 \\
6986.6 \\
7438.9\end{array}$ & $\begin{array}{ll}7 & 325.0^{\dagger} \\
6 & 866.5 \\
7 & 052.7 \\
7 & 318.6\end{array}$ & $\begin{array}{r}213.3^{\dagger} \\
170.5 \\
66.0 \\
-120.3\end{array}$ \\
\hline $\begin{array}{r}2009 \text { Q1 } \\
\text { Q2 }\end{array}$ & $\begin{array}{l}1582.7 \\
1607.2\end{array}$ & $\begin{array}{l}1.9 \\
2.7\end{array}$ & $\begin{array}{l}1584.7 \\
1609.9\end{array}$ & $\begin{array}{l}5.0^{\dagger} \\
5.5\end{array}$ & $\begin{array}{l}7198.6 \\
6725.6\end{array}$ & $\begin{array}{l}7037.1 \\
6655.9\end{array}$ & $\begin{array}{r}-161.5 \\
-69.7\end{array}$ \\
\hline \multicolumn{6}{|c|}{$\begin{array}{l}1 \text { Monetary financial institutions } \\
2 \text { Money market instruments } \\
3 \text { All loans secured on dwellings and all finance leasing are treated as long } \\
\text { term loans } \\
4 \text { Other than direct investment loans, loans secured on dwellings and loans } \\
\text { for finance leasing }\end{array}$} & \multicolumn{2}{|c|}{$\begin{array}{r}\text { Sources: National Statistics; } \\
\text { Bank of England }\end{array}$} \\
\hline
\end{tabular}




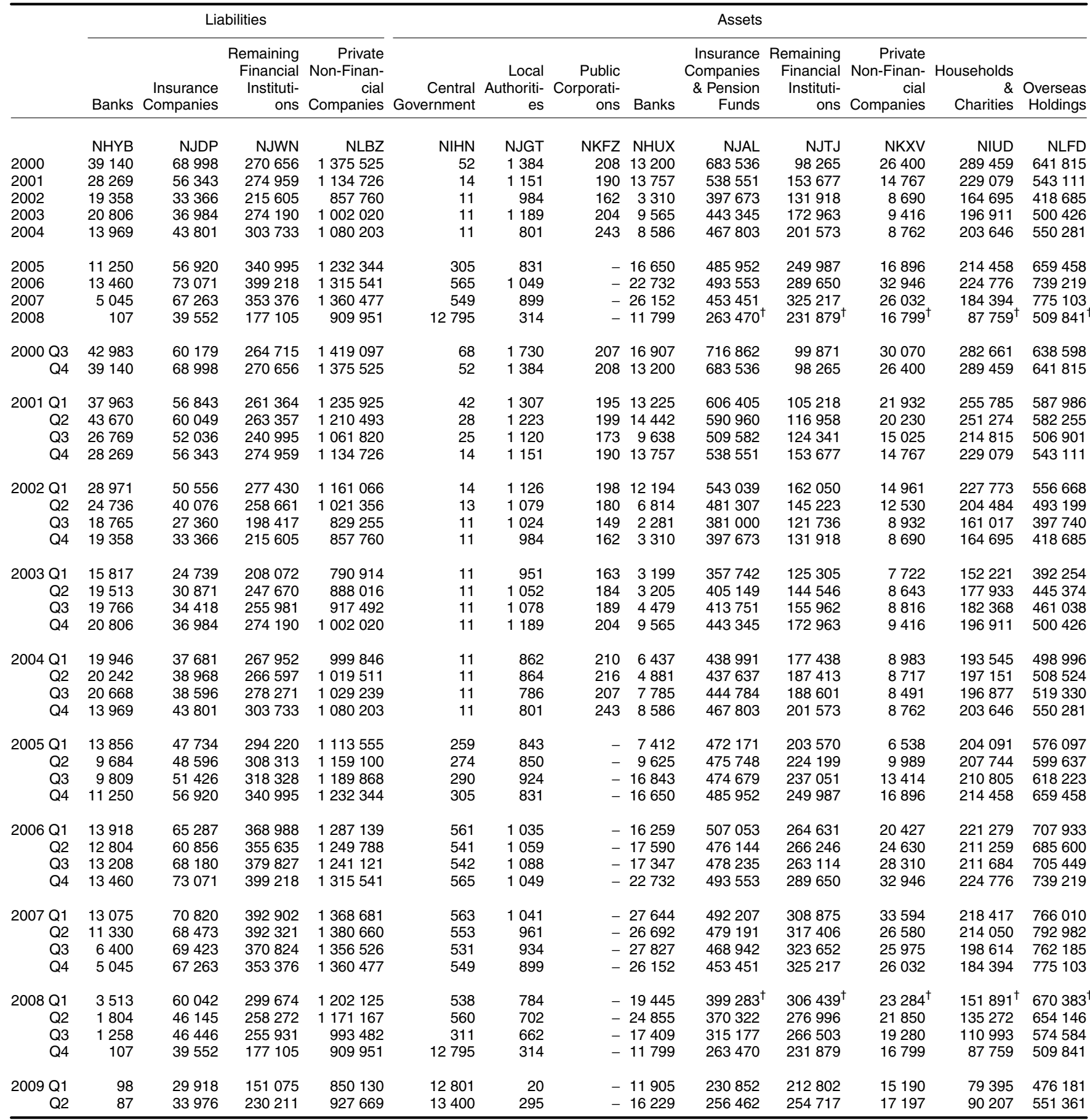

